\title{
Mapping the influence of social threat in the brain
}

Citation for published version (APA):

Engelen, T. (2018). Mapping the influence of social threat in the brain: from action observation to body ownership. [Doctoral Thesis, Maastricht University]. Maastricht University. https://doi.org/10.26481/dis.20180516te

Document status and date:

Published: 01/01/2018

DOI:

10.26481/dis.20180516te

Document Version:

Publisher's PDF, also known as Version of record

\section{Please check the document version of this publication:}

- A submitted manuscript is the version of the article upon submission and before peer-review. There can be important differences between the submitted version and the official published version of record.

People interested in the research are advised to contact the author for the final version of the publication, or visit the DOI to the publisher's website.

- The final author version and the galley proof are versions of the publication after peer review.

- The final published version features the final layout of the paper including the volume, issue and page numbers.

Link to publication

\footnotetext{
General rights rights.

- You may freely distribute the URL identifying the publication in the public portal. please follow below link for the End User Agreement:

www.umlib.nl/taverne-license

Take down policy

If you believe that this document breaches copyright please contact us at:

repository@maastrichtuniversity.nl

providing details and we will investigate your claim.
}

Copyright and moral rights for the publications made accessible in the public portal are retained by the authors and/or other copyright owners and it is a condition of accessing publications that users recognise and abide by the legal requirements associated with these

- Users may download and print one copy of any publication from the public portal for the purpose of private study or research.

- You may not further distribute the material or use it for any profit-making activity or commercial gain

If the publication is distributed under the terms of Article $25 \mathrm{fa}$ of the Dutch Copyright Act, indicated by the "Taverne" license above, 


\title{
Mapping the influence of social threat in the brain
}

from action observation to body ownership

\author{
DISSERTATION
}

to obtain the degree of Doctor at the Maastricht University, on the authority of the Rector Magnificus, Prof. Dr. Rianne M. Letschert, in accordance with the decision of the Board of Deans,

to be defended in public on Wednesday 16 May 2018, at 12:00 hours

by

Tahnée Engelen 


\section{Supervisors:}

Prof. dr. Beatrice de Gelder

Prof. dr. Alexander T. Sack

\section{Assessment committee:}

Prof. dr. Sonja Kotz (chair)

Prof. dr. Rainer Goebel

Dr. Amanda Kaas

Prof. dr. Karin Roelofs, Radboud University Nijmegen \& Donders instituut

Dr. Dennis Schutter, Radboud University Nijmegen \& Donders instituut 


\section{Contents}

Chapter 1 General introduction 5

$\begin{array}{lll}\text { Chapter } 2 \text { A causal role for inferior parietal lobule in } & 27\end{array}$ emotion body perception

Chapter 3 Dynamic interactions between emotion 45 perception and motor preparation networks for reacting to social threat: a cTBS-fMRI study

Chapter 4 Modulation of motor cortex excitability in response to consciously and unconsciously perceived whole body expressions of threat

Chapter 5 Phase of beta-frequency tACS over primary motor cortex modulates corticospinal excitability

Chapter 6 Affective vocalizations influence body ownership as measured in the Rubber Hand Illusion

Chapter 7 Gestalt-like reappearance of visual afterimages induced by early visual cortex TMS

Chapter 8 General discussion

Knowledge valorization

Acknowledgements

171

CV \& Publications

175 

Chapter 1 General Introduction 

As humans, we are capable of expressing our emotions in a vast array of ways, such as a subtle smile to signal amusement, a faint sigh to signal exasperation, or maybe a slightly raised eyebrow to signal discontent. When perceived in others, interpreting such signals might not always be straightforward, and we will often find ourselves trying to navigate the complex world of socially appropriate responses to others. A smile in response to someone expressing sadness over their recently deceased dear pet turtle is a faux-pas you are not likely to forget anytime soon. Nonetheless, whereas social embarrassment is obviously far from enjoyable, these misinterpretations are hardly life-threatening. The story becomes exceedingly different once we start exploring emotions that signal threat, such as anger and fear. Misinterpretation on the intention of someone storming at you with raised fists will surely end as more than just an awkward anecdote. Luckily, humans have become experts in emotion recognition in instances in which it truly matters.

The important role of emotions in our survival was already highlighted by Darwin, who argued for a role of emotions in triggering adaptive actions in others (Darwin, 1872). Indeed, responding appropriately to threat signals, stemming not only from the environment but also from our peers, is crucial for survival. When noticing threat expressed through others, we have many clues to go by, including a range of auditory signals like vocalizations. Take the example of primate vocalization, in which alarm calls are used to alert group members of nearby predators. Other effective ways of expressing threat emotions, which humans rely upon very strongly, are our facial expression and our body posture.

Interestingly, despite emotion expressions being so multi-faceted, there has been a traditional bias in the neuroimaging literature towards the study of facial emotion expressions. But more recently, there has been a shift in the field towards a more comprehensive understanding of emotion expressions. A greater understanding of how we read emotion from the body, and how we are influenced by these signals as observers, has emerged. Nonetheless, there are still caveats in our understanding in how we process whole body expressions of emotions, some of which I try to address in this thesis. I will start with a general introduction about what we have learned about emotion body processing so far, with emphasis on the relation between emotions and action.

\section{Distinguishing threatening from neutral actions}

Expressing an emotion with our body involves movement of our arms, our trunk and often also our legs. This makes the question relevant of how we distinguish movements that are signaling emotions, and movements that don't. For example, both when wanting to shake someone else's hand, as well as when trying to punch someone, will we bring up our hand toward someone else, however with vastly different intentions. Importantly, in one case fast and appropriate identification of the content of the action is required. Therefore, one of the first interesting questions concerning whole body expressions of emotions concerns where the dissociation is made between actions that signal threat, and actions that don't.

\section{Emotion in the Action Observation Network}

When observing actions in other humans, the so called 'action observation network' (AON) gets activated. The action observation network comprises of areas such as the superior temporal sulcus (STS), ventral premotor cortex (PMv), supplementary motor area (SMA) and 
inferior parietal lobule (IPL) (Buccino et al., 2001; Caspers, Zilles, Laird, \& Eickhoff, 2010; Decety et al., 1997; Grafton, Arbib, Fadiga, \& Rizzolatti, 1996). A major role of the AON is the understanding of actions and their underlying intentions (Fogassi et al., 2005; Iacoboni, Molnar-Szakacs, Gallese, Buccino, \& Mazziotta, 2005; Jellema, Baker, Wicker, \& Perrett, 2000; Rizzolatti, Fogassi, \& Gallese, 2001).

Accumulating evidence now suggests that in addition to this, some of the areas within the AON also serve a role in the processing of emotions. Specifically looking at whole body expressions of emotions, stronger activations for emotional versus neutral body actions have been found in several nodes of the AON, including STS, premotor cortex, and parietal areas (Grèzes, Pichon, \& de Gelder, 2007; Kret, Pichon, Grèzes, \& de Gelder, 2011; Kret, Pichon, Grèzes, \& de Gelder, 2011; Pichon, de Gelder, \& Grezes, 2008; Pichon, de Gelder, \& Grèzes, 2009). de Gelder (2006) proposed a 'two-systems' model for the processing of emotional body language, consisting of an automated reflex-like subcortical circuit and a cortical circuit in charge of recognition and deliberation. It is this cortical circuit which consists of areas such as STS, premotor, and parietal cortex which is involved in the recognition of emotions from the body and forming an appropriate response. Nonetheless, questions still remain which of these cortical action areas are causally involved in the perception of emotion bodies. Transcranial Magnetic Stimulation (TMS) is a particularly appropriate method to address the functional role of different brain areas in emotion body processing. Results of recent TMS studies are discussed in the following paragraph.

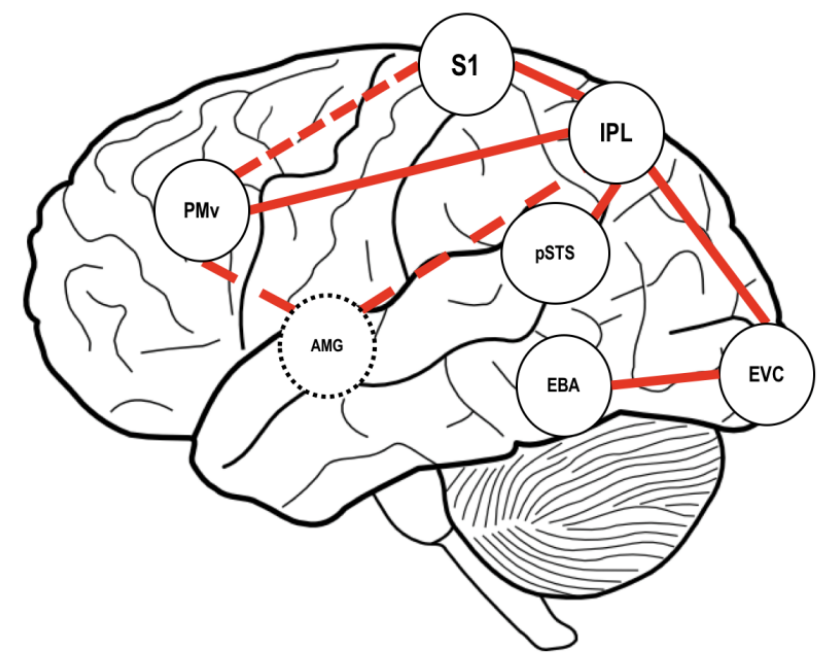

Figure 1. The network involved in the perception of emotion bodies. Visual information enters the cortex at the stage of early visual cortex (EVC), from where it gets passed on to the ventral and dorsal visual pathways. Extrastriate body area (EBA) is proposed to be involved in configural body processing. Posterior superior temporal sulcus (pSTS), inferior parietal lobule (IPL), and primary somatosensory cortex (S1) activate in response to the observation of (dynamic) whole body stimuli. Ventral premotor cortex (PMv) serves a role in both observation and preparation of actions. Amygdala (AMG) is an important site of emotion processing. 


\section{Using Transcranial Magnetic Stimulation to identify emotion specificity in the Action Observation Network}

In order to demonstrate causal involvement of AON areas in emotion body perception, several studies have combined tasks involving emotion stimuli with the application of TMS. One experiment looked specifically at two nodes of the AON, namely pSTS and PMv (Candidi, Stienen, Aglioti, \& de Gelder, 2011). While participants performed a delayed match-to-sample task involving neutral and fearful body postures, either extrastriate body area (EBA), pSTS, or $\mathrm{PMv}$ were stimulated. Stimulation of either EBA or PMv resulted in no changes in performance of the participants, whereas pSTS stimulation led to an increase in behavioural accuracy, specifically when fearful bodies were presented.

Similarly, a recent study found impaired recognition of emotions from point-light-displays after cTBS stimulation of pSTS compared to vertex stimulation (Basil, Westwater, Wiener, \& Thompson, 2017). Another TMS study focusing on emotion specificity in AON nodes used a TMS adaptation paradigm, which allows drawing conclusions about neural representations within a stimulated region (Mazzoni, Jacobs, Venuti, Silvanto, \& Cattaneo, 2017). The goal of the experiment was to see whether pSTS and anterior intraparietal sulcus (aIPS) hold neural representations of adapted stimuli compared to a control site (early visual cortex). They did this by assessing whether stimulation of these areas would lead to either an abolishment or reversal of an adaptation effect. There was no change in the adaptation effect after stimulation of pSTS, despite the fact that this area has previously been linked to processing of biological motion. In aIPS, conversely, the adaptation effect was abolished and even showed a trend for a reversal in the effect (i.e. facilitation in the incongruent stimulus condition). This effect was specific to when point-light-displays depicting fearful whole body movements were used as adapters, compared to a happy category.

\section{Evidence for parietal cortex involvement in emotion processing}

Among the areas that comprise the AON, parietal cortex (and specifically inferior parietal lobule (IPL)) might form a particularly interesting hub where observation of visual emotion stimuli (such as emotion bodies) is transformed into an action response. Besides receiving input from the visual system (Caspers et al., 2011), and its connectivity to premotor cortex (Hoshi \& Tanji, 2007; Makris et al., 2005; Mars et al., 2011), there is also evidence from animal research showing connections with the amygdala (Clower, West, Lynch, \& Strick, 2001). It is thus not surprising that, besides the aforementioned study by Mazzoni et al. (2017), there are several fMRI experiments that have found a link between parietal cortex and the processing of affective information (Goldberg, Christensen, Flash, Giese, \& Malach, 2015; Goldberg, Preminger, \& Malach, 2014; Grèzes et al., 2007; Kana \& Travers, 2012; Kitada, Johnsrude, Kochiyama, \& Lederman, 2010; Sarkheil, Goebel, Schneider, \& Mathiak, 2013). Additionally, one MEG study found fast involvement of IPL at $80-110 \mathrm{~ms}$ post stimulus onset in response to fearful compared to neutral body postures (Meeren, Hadjikhani, Ahlfors, Hämäläinen, \& de Gelder, 2016). Another study, which involved a patient group that has a lesion specific to the basolateral amygdala (Urbach-Wiethe), showed that when face-body compound stimuli portrayed fear in the body (irrespective of the emotion presented in the face), greater IPL activation was observed (Hortensius et al., 2017). 


\section{Responding to social threat; fight, flight, or freeze}

Once an action has successfully been identified as carrying a threat, the next step is preparing an appropriate response based on this information. When someone is about to attack you, standing still is not be the best option, whereas in other situations of threat, a moment of freezing to evaluate the situation might actually be beneficial. So, the next question is, when and how do we respond to the perception of social threat.

\section{Physiological responses to threat}

When confronted with immediate threat, our body will automatically react in a freeze, flight, or fight response. These responses are mediated through the autonomic nervous system, which comprises of a sympathetic and parasympathetic branch. It is the balance between these two branches which determines whether the response to threat will be action (fight/flight) or inaction (freeze). Freezing behaviour will be observed if there is a dominance of the parasympathetic nervous system, and usually results in physiological changes such a deceleration of heart rate, whereas the fight/flight response associated with sympathetic nervous system dominance would result in heart rate acceleration (Roelofs, 2017). Which of these responses to threat are triggered is dependent on several factors, such as distance and ambiguity of the threat, with distal and ambiguous threats being more likely to elicit freezing behaviour. Next to an influence of state, there are certain traits, such as aggression or trait anxiety, which can also play a role in determining which defensive response is triggered.

Whereas freezing behaviour in animals can sometimes be easily observable (imagine for example the extreme case of fainting goats), humans are similarly capable of displaying such a defensive response (Hagenaars, Oitzl, \& Roelofs, 2014). Evidence for human freezing behaviour, specifically in response to threat signalled by others, comes from experiments measuring heart rate and body sway. For example, when being presented with faces expressing anger, a reduction in both heart rate (bradycardia) and body sway can be observed compared to happy and neutral faces (Roelofs, Hagenaars, \& Stins, 2010). Further work suggests that responses such as bradycardia and reduced body sway are subject to whether the recipient of the threat has the ability to respond or not (Gladwin, Hashemi, van Ast, \& Roelofs, 2016), linking the concept of freezing to action preparation.

\section{Changes in motor cortex excitability in response to emotion observation}

Another way of exploring defensive responses in humans is by assessment of the state of the motor system. One direct way of accomplishing this is by administering single TMS pulses over M1, and subsequently using electromyography (EMG) to measure the amplitude of the elicited Motor Evoked Potential (MEP). This amplitude reflects the excitability of the motor cortex and can be interpreted as readiness to make a motor response (Bestmann \& Krakauer, 2015; Rothwell, 1997). If indeed a certain threatening stimulus triggers a fight/flight or freeze response in the observer, this should be reflected in respective increases or decreases in the amplitude of the MEP compared to a control condition. Using this method, it has so far been demonstrated that changes in amplitude of MEPs can be observed when participants are presented with affective scenes (Coelho, Lipp, Marinovic, Wallis, \& Riek, 2010; Coombes et al., 2009; Hajcak et al., 2007; Lagravinese et al., 2017; van Loon, van den Wildenberg, van Stegeren, 
Ridderinkhof, \& Hajcak, 2010), as well as in response to faces expressing emotions (Schutter, Hofman, \& Van Honk, 2008).

The study of how bodily expressions of emotion modulate readiness of the motor cortex in order to respond to threat has led to several interesting insights. Firstly, it was demonstrated that, like affective scenes and faces, emotion bodies are capable of altering MEP amplitude at $300 \mathrm{~ms}$ post stimulus onset, but this modulation was similar to that caused by neutral actions (Borgomaneri, Gazzola, \& Avenanti, 2012). Another study looking at the same time point and looking at dynamic whole body movements, rather than static, showed that compared to both neutral and fear expressions, expressions of anger lead to increases of MEP amplitude, irrespective of whether these movements were directed towards or away from the observer (Hortensius, de Gelder, \& Schutter, 2016). In terms of responding to threat, 300ms might be considered rather slow, and other studies therefore also explored earlier time points. What was found is that already at $70-90 \mathrm{~ms}$ after the onset of stimuli consisting of bodies expressing fear, increases of MEP amplitude can be observed. At $100-125 \mathrm{~ms}$ post stimulus onset this MEP modulation to fear is abolished, and changes in threat response are rather expressed through intracortical facilitation (Borgomaneri, Vitale, \& Avenanti, 2017; Borgomaneri, Vitale, Gazzola, \& Avenanti, 2015). This is in line with the finding that at $150 \mathrm{~ms}$ post stimulus onset there is a suppression, rather than increase, of the MEP amplitude (Borgomaneri, Gazzola, \& Avenanti, 2014). Together, these findings suggest that the readiness of the motor cortex can vary between fight/flight and freeze responses depending on the amount of time that has passed since the threat was perceived, but which might also depend on personality traits like behavioural inhibition/activation (Borgomaneri et al., 2017), or depending on the ambiguity of the threat (e.g. the difference between anger and fear).

\section{Unconscious processing of emotional bodies}

\section{Unconscious emotion processing in the brain}

Given the critical role in triggering survival behaviour, it is not surprising that the influence of emotion perception has been shown to also reach outside of conscious awareness. Valuable evidence for this stems from research looking into so called 'affective blindsight', which can be observed in certain cortically blind patients. This phenomenon refers to the uncanny ability of identifying emotions in facial stimuli above chance level, despite them being presented in the blind area of the visual field (scotoma) of the patient (Morris, de Gelder, Weiskrantz, \& Dolan, 2001). Similar findings have been obtained in healthy participants by means of TMS stimulation. Jolij \& Lamme (2005) managed to mimic affective blindsight by using TMS over $\mathrm{V} 1$ to disrupt visual processing and showing that participants were still able to identify the emotion expressed by a face presented in the part of the visual field affected by the TMS above chance level. In the brain, many subcortical areas have been found to be involved in the processing of unconscious emotional information. Of these structures, the superior colliculus, pulvinar and the amygdala have been repeatedly demonstrated to activate in response to unconsciously presented fearful faces, both in healthy participants (Liddell et al., 2005; Morris, Ohman, \& Dolan, 1999; Whalen et al., 1998; Whalen et al., 2004; Williams, 2004), but also in patients displaying affective blindsight (Morris et al., 2001; Pegna, Khateb, Lazeyras, \& Seghier, 2005; Van den Stock et al., 2014). Together, these areas form a subcortical pathway whereby such visually presented expressions of emotions can be non-consciously processed (Tamietto $\&$ de Gelder, 2010). The pulvinar as such has been implicated in saliency detection (Robinson 
\& Petersen, 1992), the amygdala is a well-known centre for emotion processing (Davis \& Whalen, 2001), and the superior colliculus has been linked to triggering responses to emotion stimuli (Schenberg et al., 2005).

\section{Physiological and behavioural changes in response to unconscious emotions}

Other than responsiveness of emotion sensitive brain areas to unseen emotional stimuli, effects can also be observed in changes in physiology and behaviour. For example, in patients with affective blindsight, both changes in pupil size, as well as synchronization of facial expressions can be observed in conditions of unconscious emotion perception (Tamietto et al., 2009), findings which have also been demonstrated in healthy participants (Dimberg, Thunberg, \& Elmehed, 2000; Tamietto \& de Gelder, 2008). Other reported physiological modulations include skin conductance (Esteves, Dimberg, \& Öhman, 1994), heart rate (Ruiz-Padial, Mata, Rodríguez, Fernández, \& Vila, 2005; Ruiz-Padial, Vila, \& Thayer, 2011), and changes in levels of stress hormones (Van Honk et al., 1998). In terms of behavioural effects, nonconscious presented emotion stimuli range from shifts in covert spatial attention (Carlson \& Reinke, 2008) to influencing likeability of novel items (Almeida, Pajtas, Mahon, Nakayama, \& Caramazza, 2013).

\section{Evidence for processing of unconscious emotional information conveyed by the body}

Like faces, bodies conveying emotions have also been subject to the study of unconscious emotion processing. Affective blindsight does not seem to be restricted to emotion conveyed by the face, as dynamic whole body expressions of emotion can also be identified above chance level (Van den Stock et al., 2011). One fMRI study involving a patient with unilateral V1 damage showed activation of area MT and pulvinar in response to happy, but not neutral body images (de Gelder \& Hadjikhani, 2006). Likewise, using TMS over V1, it was demonstrated in healthy participants that while discrimination of neutral body images at phosphene location could be impaired by stimulation, discrimination of emotional postures remained intact (Filmer \& Monsell, 2013). What is particularly interesting, is that one study involving a cortically blind patient showed that whole body expressions of anger that are unconsciously perceived are capable of activating cortical areas. Specifically, they found activations in somatosensory, premotor and motor areas, in line with the idea that whole body expressions of emotions are strong triggers for action preparation (Van den Stock et al., 2011).

As has been demonstrated with facial stimuli, unseen emotion bodies can also elicit activation of facial muscles that are congruent with the emotion that is being portrayed (Tamietto et al., 2009), which in addition to giving evidence of nonconscious processing of emotion bodies, also shows that such responses are more than simple mimicry. Additionally, exposure to unconscious fear bodies increase pupil dilation in a similar way to consciously perceived fear bodies (Tamietto et al., 2015).

In terms of behavioural responses to unconsciously presented emotional bodies, one study used a continuous flash suppression paradigm as a method of rendering bodies invisible, and then measured the time it took before the participant became aware of the stimulus (breaking from suppression (b-CFS)) (Zhan, Hortensius, \& de Gelder, 2015). Stimuli consisted of body postures that were neutral, angry, or fearful. Results indicated that while suppression time for angry bodies were significantly shorter compared to neutral bodies, this pattern was reversed 
for fearful versus neutral bodies. This pattern of suppression times is particularly interesting with respect to fight/flight versus freeze behaviour as discussed in the previous section, as perceiving fear and anger in others might have opposing effects in the observer due to differences in ambiguity (fear being more ambiguous than anger).

\section{Beyond reflexive responses to emotion; the influence of emotion perception on our bodily sense of self}

So far, we have seen that perceiving threat in others is tightly linked to both the observation and preparation of actions, and that such threat signals have preferential access to consciousness. The next question that arises is whether the influence of social threat signals ends there, or if there is reason to believe their influence can resonate beyond reflexive responses and extend to concepts concerning our sense of self.

\section{Defining our bodily sense of self}

Our 'sense of self' is a very broad concept, and there are certainly many elements of our experiences contributing to our innate sense that we are indeed ourselves. One way of approaching the topic is by considering the building blocks that form our sense of self, or bodily self-consciousness (Blanke, 2012), as whatever we attribute as being part of our body, we will also consider as being part of ourselves. In terms of bodily self-consciousness, there are two main concepts that seem to capture most of the essence of what constitutes the experience of having and belonging in a body.

The first one is called 'agency' and refers to the feeling that we are in control of moving our own body. E.g. if I now initiate movement with my hand, the feeling that it is me who is moving the hand is an essential construct to also experience this hand as my own. In other words, agency refers to the feeling that I am in control of moving my body. Interestingly, agency is not always a given. Take for example the case of anarchic hand syndrome. This syndrome is characterized by uncontrollable movements of the hand (Marchetti \& Della Salla, 1998). Notably, these movements are often not just simple in their nature, but can also manifest in rather complex instrumental movements. Patients suffering from this syndrome do not feel in control of these movements, and will thus not experience a sense of agency over the affected hand. What is striking is that they will still report the feeling that the anarchic hand belongs to their body, which brings us to the second important component of bodily self-consciousness, namely 'body ownership'.

Body ownership refers to the feeling that a certain part of the body belongs to you. I can keep my hand in front of me on the table without moving it (so essentially not evoking a sense of agency), and still feel like the hand I see belongs to me. The same would hold if someone else would now lift my hand from the table; I myself would not be moving my hand and thus no experience of agency would arise, yet I could still experience ownership over the hand that is being lifted. Like agency, ownership is not always a given. For example, sometimes a lesion of the temporo-parietal junction can result in somatoparaphrenia, which is a disorder in which patients can lose their sense of ownership of a limb, and sometimes even misattribute the limb as belonging to a different person (Halligan, Marshall, \& Wade, 1995; Vallar \& Ronchi, 2009). It is not just in certain patient groups in which misattributions of limbs are sometimes made, as it turns out it is quite simple to induce 'errors' in body ownership in healthy people as well 
(see the example of the rubber hand illusion below). This tells us that body ownership is malleable, which begs the question of whether being under threat might alter the boundaries of ownership as well.

\section{Peri-personal space as a malleable boundary between threat and the self}

So far, there are not many studies investigating either agency or ownership in direct relation to social threat perception. One component relating to our bodily self that has been studied in relation to social threat, however, is peri-personal space (PPS). PPS refers to the space closely surrounding our body (Rizzolatti, Scandolara, Matelli, \& Gentilucci, 1981), which is represented in brain areas integrating multisensory signals (Brozzoli, Gentile, Petkova, \& Ehrsson, 2011). PPS represents objects near our body with which we could interact, as well as forming a boundary in social interactions that we do not want to have crossed. PPS is anchored to the body and its boundaries are flexible, having the ability to expand or contract in different situations (Brozzoli, Cardinali, Pavani, \& Farnè, 2010; Cardinali et al., 2009; Farnè \& Làdavas, 2000). PPS is sensitive to possible threats close to our body, which is why we will typically have attention allocated to monitoring this space (Coello, Bourgeois, \& Iachini, 2012). With regard to responding to threats in the environment, our PPS has been termed a 'protective space' (Sambo \& Iannetti, 2013), reflecting the notion that it is critical to be aware of what poses an immediate threat (Graziano \& Cooke, 2006).

With respect to whether emotions influence PPS boundaries, it has been shown in the visual domain that time-to-collision is judged as shorter for threatening versus non-threatening stimuli (Vagnoni, Lourenco, \& Longo, 2012), and likewise in the auditory domain, sounds with negative valence lead to an expansion of personal space (Tajadura-Jiménez, Pantelidou, Rebacz, Västfjäll, \& Tsakiris, 2011). Recent work by Ferri, Tajadura-Jiménez, Väljamäe, Vastano, \& Costantini (2015) showed how emotion-inducing looming sounds can modify the boundaries of multisensory PPS. Their results showed that emotions can change PPS boundaries and that, whereas negative looming-sounds can lead to an expansion of PPS, looming sounds of a positive valence did the opposite and led to shrinking of PPS boundaries. Aside from emotional looming sounds, there is also an influence of both context and closeness of the hand to the face on the hand-blink reflex (Sambo, Forster, Williams, \& Iannetti, 2012). In addition, interindividual differences in PPS extension are positively related to trait anxiety (Lourenco, Longo, \& Pathman, 2011; Sambo \& Iannetti, 2013).

\section{Manipulating multisensory signals to study bodily self-consciousness}

One way of investigating bodily self-consciousness is by presenting participants with conflicting multisensory information for example about the location of one of their limbs. Accomplishing such incongruent multisensory stimulation can be rather challenging. Let's take hand ownership as an example. Ownership of our hands is build out of integration of multiple senses; our hands are often in our field of view, and next to this visual feedback we also receive feedback on the location of our hands through proprioception, as well as information from anything we touch with our hands through tactile feedback. Proper incongruence would then mean making someone believe that their hand is in a different location than where they see or feel their hand. Progress in virtual reality and robotics has certainly made such manipulations more feasible (Blanke, 2012), but there are also other manipulations that can achieve similar things. Two of these paradigms will be discussed below. 


\section{The Rubber Hand illusion}

The Rubber Hand Illusion (RHI) refers to an illusion in which a false sense of ownership over a fake hand can be elicited (Botvinick \& Cohen, 1998). This illusion can be created by inducing a conflict between visual and proprioceptive feedback of the hand. During the induction of the illusion, a realistic fake hand is placed in an anatomically plausible position in front of a participant, while the participant's real hand is occluded from view. When the real hand and fake hand are then stroked in synchrony, participants will report feelings of ownership over the fake hand. The illusion can be captured in a subjective report, as well as a proprioceptive drift of the experienced location of their real hand towards the fake hand. The RHI is an example in which multisensory conflict is resolved by one sense capturing the others. In this case it is vision that dominates proprioception, something that can also be observed in studies using prism glasses. When using prism glasses the sensed location of a participant's hand gets pulled toward the displaced visual representation of the hand (Hay, Pick, \& Ikeda, 1965). This capturing of vision over other senses is often attributed to the higher spatial acuity of vision compared to the other senses, however as we will see later vision can also be dominated by other senses. The RHI already illustrates that body ownership is malleable, and several things have been demonstrated to alter the strength of the illusion. For example, people with lower interoceptive sensitivity experience a stronger illusion compared to people with higher interoceptive sensitivity (Tsakiris, Tajadura-Jiménez, \& Costantini, 2011), showing the influence of not just exteroceptive but also interoceptive signals.

\section{Positive Afterimages}

If a conflict occurs between multiple senses, it seems intuitive that vision would dominate the other. As we saw in the case of the RHI, vision dominates proprioception when a conflict between the two is induced. This provides an example of how vision can influence bodily selfconsciousness (Faivre, Salomon, \& Blanke, 2015). Nonetheless, there are also cases in which the opposite can be observed, i.e. vision being captured by proprioception and thus bodily selfconsciousness shaping vision. One such example comes from positive afterimages. Most people will be familiar with the concept of an afterimage, and positive afterimages are very similar in that they are images which remain in your vision even after the original image has disappeared, but with the difference that they look exactly as they were perceived before this disappearance (hence the 'positive'). Positive afterimages can be elicited by having the eyes first fully adapt to a dark room. If, after this dark adaptation, the eyes are very briefly exposed to a flash, an afterimage will develop on the retina which looks exactly as whatever was perceived and illuminated during the flash. After a brief dark period, the afterimage slowly develops and builds up to a clear percept, and after several seconds it slowly fades out. This means that if one of your limbs was in your field of view while the flash went off, you will develop an afterimage in which this limb is present. Because afterimages can last for multiple seconds before they fade away, this creates and interesting opportunity to create a conflict between where you see your limb in the afterimage versus where you feel the location of your limb in real time (Davies, 1973). What is so peculiar is that in this case the conflict induced between vision and proprioception is resolved by changing the percept of the observer.

One such example can be observed during the Taylor illusion. If during the brief exposure of the flash, you are holding your hand in front of your face, then an afterimage would develop which would also contain an image of the hand. If, after the development of the afterimage, 
the hand is moved either closer to or away from the face, an expansion or shrinking of the hand present in the afterimage can be observed (Carey \& Allan, 1996). The direction of size change of the hand will follow Emmert's law, which states that a retinal image is proportional in size to the distance of the surface it is projected upon. The scaling of the size of the afterimage occurs irrespective of whether the movement of the hand was made actively or passively, which shows that afferent proprioceptive information is sufficient to influence the visual percept (Bross, 2000). Hand ownership is also a key factor in the extent to which the visual percept can be changed. Faivre et al. (2017) used a combination of positive after images and a version of the RHI called the somatic rubber hand illusion. In this variation of the RHI, an illusion of self-touch is created by having a participant touch a fake hand with their right hand, while at the same time their left hand is being touched in synchrony by the experimenter (Ehrsson, Holmes, \& Passingham, 2005). In the experiment by Faivre et al. (2017) they tested whether drifts in the afterimage of an illusory owned hand occur when the location of the real hand (and thus proprioceptive feedback) is kept constant. They found that the drift of the afterimage was dependent on experienced ownership over the fake hand (i.e. a drift of the afterimage towards the subjectively owned hand), showing a role of ownership in the Taylor illusion.

There's another instance in which the percept of the afterimage of a hand can be altered. If after the development of the afterimage the hand is fully removed from the field of view, you create a situation in which proprioception and vision are signaling opposite information, namely if you at that point in time should be seeing your hand or not. What happens when such a conflict is created is that the hand present in the afterimage will fade, or crumble, as a result of the movement (Davies, 1973; Gregory, Wallace, \& Campbell, 1959). Note that it is not a general fading of the afterimage that occurs as a result of the conflict; if both hands were present in the afterimage and only one is removed, it is only the removed hand that will fade, whereas everything else in the afterimage will remain clearly visible. Again, ownership of the hand plays a crucial role. Hogendoorn, Kammers, Carlson, \& Verstraten (2009) tested this by having the hand removed from the field of view either in the dark period, before the afterimage has developed, or after the development of the afterimage. If the hand is moved during the dark period, the incongruence between vision and proprioception already occurs before the development of the afterimage, and if the hand is then moved again later on, no fading occurs. Like with the Taylor illusion, fading of the hand occurs irrespective of whether the movement is active or passive (Hogendoorn et al., 2009).

\section{Outline of the thesis}

This thesis consists of three main parts. Part 1 revolves around the question whether action observation areas are involved in the processing of social threat information, specifically conveyed through whole body emotion stimuli. In Chapter 2 we focus on inferior parietal lobule (IPL) and use online TMS to establish a causal relevance of IPL in emotion body processing. By using triple pulse TMS while participants perform a delayed-match-to-sample task that involves trials presenting either neutral or fearful bodies, we can observe changes in behavioural performance depending on stimulation of either IPL, or one of the control conditions (stimulation of extrastriate body area, early visual cortex, or sham stimulation). In Chapter 3 we continue to investigate the role of IPL in emotion body processing by using a combination of offline continuous theta burst stimulation (cTBS) and fMRI acquisition. Additionally, in this chapter the role of ventral premotor cortex is considered. The combination of TMS and fMRI allows for investigating network involvement and interactions between brain areas while 
emotion body information is being processed. The set-up of this experiment also allowed for the use of dynamic, rather than static, whole body stimuli.

In Part 2 of the thesis, the main focus is on the motor system. In Chapter 4 we investigate how the motor system, and specifically corticospinal excitability as measured with motor evoked potentials (MEPs), is affected by the presentation of emotion body stimuli. The goal is to establish how and when MEPs are modulated by subliminally or supraliminally presented emotion body stimuli, in order to get a better understanding on the conditions under which action preparation in response to threat occurs. At the end of chapter 3 we discuss issues with reliability of MEP measurements, and therefore in Chapter 5 we investigate two potential sources underlying MEP variability; namely power and phase of neuronal oscillations. We tackle this question by using an online combination of transcranial alternating current stimulation (tACS) with phase-locked single pulse TMS. We then apply tACS at individual alpha, individual beta or sham stimulation and analyse online phase effects, as well as offline power effects of these frequency bands.

Lastly, Part 3 of the thesis focuses on different aspects of bodily self-consciousness. First, in Chapter 6, we look into if and how body ownership is influenced by social threat. To answer this question, we use a well-established paradigm for measuring body ownership, namely the rubber hand illusion, and combine it with the presentation of auditory emotion stimuli (affective vocalizations). We then assess the amount of experienced subjective (questionnaire) and objective (proprioceptive drift) shifts in ownership in the different sound conditions (affective vocalizations, neutral vocalizations, non-vocal sounds or no sounds). Lastly, in Chapter 7, we investigate how vision is influenced by proprioception in the case of positive afterimages, and specifically whether fading of the hand from an afterimage is the result of inhibition of early visual cortex. We use a positive afterimages paradigm in which fading of the hand from the afterimage is induced through movement of the hand. We then assess whether this fading originates from inhibition of early visual cortex (EVC) by releasing EVC from this inhibition using online TMS. As the outcome measure we measure if the amount of experienced fading of the removed hand was altered by the stimulation. 


\section{References}

Almeida, J., Pajtas, P. E., Mahon, B. Z., Nakayama, K., \& Caramazza, A. (2013). Affect of the unconscious: Visually suppressed angry faces modulate our decisions. Cognitive, Affective, \& Behavioral Neuroscience, 13(1), 94-101. https://doi.org/10.3758/s13415-012-0133-7

Basil, R. A., Westwater, M. L., Wiener, M., \& Thompson, J. C. (2017). A Causal Role of the Right Superior Temporal Sulcus in Emotion Recognition From Biological Motion Rochelle. Open Mind: Discoveries in Cognitive Science, 1(3), 148-158. https://doi.org/https://doi.org/ 10.1162/opmi_a_00015

Bestmann, S., \& Krakauer, J. W. (2015). The uses and interpretations of the motor - evoked potential for understanding behaviour, 679-689. https://doi.org/10.1007/s00221-014-4183-7

Blanke, O. (2012). Multisensory brain mechanisms of bodily self-consciousness. Nature Reviens Neuroscience, 13(8), 556-571. https://doi.org/10.1038/nrn3292

Borgomaneri, S., Gazzola, V., \& Avenanti, A. (2012). Motor mapping of implied actions during perception of emotional body language. Brain Stimulation, 5(2), 70-6. https://doi.org/10.1016/j.brs.2012.03.011

Borgomaneri, S., Gazzola, V., \& Avenanti, A. (2014). Transcranial magnetic stimulation reveals two functionally distinct stages of motor cortex involvement during perception of emotional body language. Brain Structure \& Function. https://doi.org/10.1007/s00429-014-0825-6

Borgomaneri, S., Vitale, F., \& Avenanti, A. (2017). Behavioral inhibition system sensitivity enhances motor cortex suppression when watching fearful body expressions. Brain Structure and Function, $O(0)$, 0. https://doi.org/10.1007/s00429-017-1403-5

Borgomaneri, S., Vitale, F., Gazzola, V., \& Avenanti, A. (2015). Seeing fearful body language rapidly freezes the observer's motor cortex. Cortex, 65, 232-245. https://doi.org/10.1016/j.cortex.2015.01.014

Botvinick, M., \& Cohen, J. (1998). Rubber hands "feel” touch that eyes see. Nature, 391(February), 756. https://doi.org/10.1038/35784

Bross, M. (2000). Emmert's law in the dark: Active and passive proprioceptive effects on positive visual afterimages. Perception, 29(11), 1385-1391. https://doi.org/10.1068/p3038

Brozzoli, C., Cardinali, L., Pavani, F., \& Farnè, a. (2010). Action-specific remapping of peripersonal space. Neuropsychologia, 48(3), 796-802. https://doi.org/10.1016/j.neuropsychologia.2009.10.009

Brozzoli, C., Gentile, G., Petkova, V. I., \& Ehrsson, H. H. (2011). fMRI Adaptation Reveals a Cortical Mechanism for the Coding of Space Near the Hand. Journal of Neuroscience, 31(24), 9023-9031. https://doi.org/10.1523/JNEUROSCI.1172-11.2011

Buccino, G., Binkofski, F., Fadiga, L., Fogassi, L., Gallese, V., Seitz, R. J., ... Freund, H.-J. (2001). Action observation activates premotor and pariental areas in a somatotopic manner: An fMRI study. Federation of European Neuroscience Societies, 13, 400-404.

Candidi, M., Stienen, B. M. C., Aglioti, S. M., \& de Gelder, B. (2011). Event-Related Repetitive Transcranial Magnetic Stimulation of Posterior Superior Temporal Sulcus Improves the Detection of Threatening Postural Changes in Human Bodies. Journal of Neuroscience, 31(48), 17547-17554. 
https://doi.org/10.1523/JNEUROSCI.0697-11.2011

Cardinali, L., Frassinetti, F., Brozzoli, C., Urquizar, C., Roy, A. C., \& Farnè, A. (2009). Tool-use induces morphological updating of the body schema. Current Biology: CB, 19(12), R478-9. https://doi.org/10.1016/j.cub.2009.05.009

Carey, D. P., \& Allan, K. (1996). A motor signal and visual size perception. Experimental Brain Research, 110(3), 482-486. https://doi.org/10.1007/BF00229148

Carlson, J. M., \& Reinke, K. S. (2008). Masked fearful faces modulate the orienting of covert spatial attention. Emotion, 8(4), 522.

Caspers, S., Eickhoff, S. B., Rick, T., Kapri, A. Von, Kuhlen, T., Huang, R., ... Zilles, K. (2011). Probabilistic fibre tract analysis of cytoarchitectonically defined human inferior parietal lobule areas reveals similarities to macaques. NeuroImage, 58(2), 362-380. https://doi.org/10.1016/j.neuroimage.2011.06.027

Caspers, S., Zilles, K., Laird, A. R., \& Eickhoff, S. B. (2010). ALE meta-analysis of action observation and imitation in the human brain. NeuroImage, 50(3), 1148-1167. https://doi.org/10.1016/j.neuroimage.2009.12.112

Clower, D. M., West, R. a, Lynch, J. C., \& Strick, P. L. (2001). The inferior parietal lobule is the target of output from the superior colliculus, hippocampus, and cerebellum. The Journal of Neuroscience, 21(16), 6283-6291. https://doi.org/21/16/6283

Coelho, C. M., Lipp, O. V., Marinovic, W., Wallis, G., \& Riek, S. (2010). Increased corticospinal excitability induced by unpleasant visual stimuli. Neuroscience Letters, 481(3), 135-138. https://doi.org/10.1016/j.neulet.2010.03.027

Coello, Y., Bourgeois, J., \& Iachini, T. (2012). Embodied perception of reachable space: how do we manage threatening objects? Cognitive Processing, 13 Suppl 1, S131-5. https://doi.org/10.1007/s10339-012-0470-z

Coombes, S. A., Tandonnet, C., Fujiyama, H., Janelle, C. M., Cauraugh, J. H., \& Summers, J. J. (2009). Emotion and motor preparation: A transcranial magnetic stimulation study of corticospinal motor tract excitability. Cognitive, Affective \& Behavioral Neuroscience, 9(4), 380-388. https://doi.org/10.3758/CABN.9.4.380

Darwin, C. (1872). The expression of the emotions in man and animals. London: John Murray. https://doi.org/10.1097/00000441-195610000-00024

Davies, P. (1973). Effects of movements upon the appearance and duration of a prolonged visual afterimage: 1. Changes arising from the movement of a portion of the body incorporated in the afterimaged scene. Perception, 2(2), 147-153.

Davis, M., \& Whalen, P. J. (2001). The amygdala : vigilance and emotion. Molecular Psycbiatry, 6(1), 13-34.

de Gelder, B. (2006). Towards the neurobiology of emotional body language. Nature Reviens. Neuroscience, 7(3), 242-9. https://doi.org/10.1038/nrn1872

de Gelder, B., \& Hadjikhani, N. (2006). Non-conscious recognition of emotional body language. NeuroReport, 17(6), 583-586. https://doi.org/10.1097/00001756-200604240-00006

Decety, J., Grèzes, J., Costes, N., Perani, D., Jeannerod, M., Procyk, E., ... Fazio, F. (1997). Brain activity 
during observation of actions. Influence of action content and subject's strategy. Brain, (120), 1763-1777.

Dimberg, U., Thunberg, M., \& Elmehed, K. (2000). Unconscious Facial Reactions to Emotional Facial Expressions. Psychological Science, 11(1), 86-89. https://doi.org/10.1111/1467-9280.00221

Ehrsson, H. H., Holmes, N. P., \& Passingham, R. E. (2005). Touching a Rubber Hand: Feeling of Body Ownership Is Associated with Activity in Multisensory Brain Areas. Journal of Neuroscience, 25(45), 10564-10573. https://doi.org/10.1523/JNEUROSCI.0800-05.2005

Esteves, F., Dimberg, U., \& Öhman, A. (1994). Automatically elicited fear: Conditioned skin conductance responses to masked facial expressions. Cognition \& Emotion, 8(5), 393-413.

Faivre, N., Dönz, J., Scandola, M., Dhanis, H., Bello Ruiz, J., Bernasconi, F., ... Blanke, O. (2017). SelfGrounded Vision: Hand Ownership Modulates Visual Location through Cortical $\beta$ and $\gamma$ Oscillations. The Journal of Neuroscience, 37(1), 11-22. https://doi.org/10.1523/JNEUROSCI.056316.2017

Faivre, N., Salomon, R., \& Blanke, O. (2015). Visual consciousness and bodily self-consciousness. Current Opinion in Neurology, 28(1), 23-28. https://doi.org/10.1097/WCO.0000000000000160

Farnè, A., \& Làdavas, E. (2000). Dynamic size-change of hand peripersonal space following tool use. Neuroreport, 11(8), 1645-1649. https://doi.org/10.1097/00001756-200006050-00010

Ferri, F., Tajadura-Jiménez, A., Väljamäe, A., Vastano, R., \& Costantini, M. (2015). Emotion-inducing approaching sounds shape the boundaries of multisensory peripersonal space. Neuropsychologia, 70 , 468-75. https://doi.org/10.1016/j.neuropsychologia.2015.03.001

Filmer, H. L., \& Monsell, S. (2013). TMS to V1 spares discrimination of emotive relative to neutral body postures. Neuropsychologia, 51(13), 2485-91. https://doi.org/10.1016/j.neuropsychologia.2013.09.029

Fogassi, L., Ferrari, P. F., Geslerich, B., Rozzi, S., Chersi, F., \& Rizzolatti, G. (2005). Parietal lobe: From action organisation to intention understanding. Science, 308(April), 662-667. https://doi.org/10.1126/science.1111199

Gladwin, T. E., Hashemi, M. M., van Ast, V., \& Roelofs, K. (2016). Ready and waiting: Freezing as active action preparation under threat. Neuroscience Letters, 619, 182-188. https://doi.org/10.1016/j.neulet.2016.03.027

Goldberg, H., Christensen, A., Flash, T., Giese, M. A., \& Malach, R. (2015). Brain activity correlates with emotional perception induced by dynamic avatars. NeuroImage, 122, 306-317. https://doi.org/10.1016/j.neuroimage.2015.07.056

Goldberg, H., Preminger, S., \& Malach, R. (2014). The emotion-action link? Naturalistic emotional stimuli preferentially activate the human dorsal visual stream. Neurolmage, 84, 254-64. https://doi.org/10.1016/j.neuroimage.2013.08.032

Grafton, S., Arbib, M., Fadiga, L., \& Rizzolatti, G. (1996). Localization of grasp representations in humans by positron emission tomography. Experimental Brain Research, 112, 103-111. Retrieved from http://link.springer.com/article/10.1007/BF00227183

Graziano, M. S. A., \& Cooke, D. F. (2006). Parieto-frontal interactions, personal space, and defensive behavior.

Neuropsychologia,

$44(13)$,

2621-2635. 
https://doi.org/10.1016/j.neuropsychologia.2005.09.011

Gregory, R. L., Wallace, J. G., \& Campbell, F. W. (1959). Changes in the size and shape of visual afterimages observed in complete darkness during changes of position in space. Quarterly Journal of Experimental Psychology, 11 (January 2015), 54-55. https://doi.org/10.1080/17470215908416288

Grèzes, J., Pichon, S., \& de Gelder, B. (2007). Perceiving fear in dynamic body expressions. NeuroImage, 35(2), 959-67. https://doi.org/10.1016/j.neuroimage.2006.11.030

Hagenaars, M. A., Oitzl, M., \& Roelofs, K. (2014). Updating freeze: Aligning animal and human research. Neuroscience and Biobehavioral Reviews, 47, 165-176. https://doi.org/10.1016/j.neubiorev.2014.07.021

Hajcak, G., Molnar, C., George, M. S., Bolger, K., Koola, J., \& Nahas, Z. (2007). Emotion facilitates action: a transcranial magnetic stimulation study of motor cortex excitability during picture viewing. Psychophysiology, 44(1), 91-7. https://doi.org/10.1111/j.1469-8986.2006.00487.x

Halligan, P. W., Marshall, J. C., \& Wade, D. T. (1995). Unilateral Somatoparaphrenia After Right Hemisphere Stroke: A Case Description. Cortex, 31(1), 173-182. https://doi.org/10.1016/S00109452(13)80115-3

Hay, J. C., Pick, H. L., \& Ikeda, K. (1965). Visual capture produced by prism spectacles. Psychonomic Science, 2(1-12), 215-216. https://doi.org/10.3758/BF03343413

Hogendoorn, H., Kammers, M. P. M., Carlson, T. a., \& Verstraten, F. a J. (2009). Being in the dark about your hand: Resolution of visuo-proprioceptive conflict by disowning visible limbs. Neuropsychologia, 47, 2698-2703. https://doi.org/10.1016/j.neuropsychologia.2009.05.014

Hortensius, R., de Gelder, B., \& Schutter, D. J. L. G. (2016). When anger dominates the mind: Increased motor corticospinal excitability in the face of threat. Psychophysiology, 53(9), 1307-1316. https://doi.org/10.1111/psyp.12685

Hortensius, R., Terburg, D., Morgan, B., Stein, D. J., van Honk, J., \& de Gelder, B. (2017). The Basolateral Amygdalae and Frontotemporal Network Functions for Threat Perception. Eneuro, ENEURO.0314-16.2016. https://doi.org/10.1523/ENEURO.0314-16.2016

Hoshi, E., \& Tanji, J. (2007). Distinctions between dorsal and ventral premotor areas: anatomical connectivity and functional properties. Current Opinion in Neurobiology, 17(2), 234-242. https://doi.org/10.1016/j.conb.2007.02.003

Iacoboni, M., Molnar-Szakacs, I., Gallese, V., Buccino, G., \& Mazziotta, J. C. (2005). Grasping the intentions of others with one's own mirror neuron system. PLOS Biology, 3(3), 0529-0535. https://doi.org/10.1371/journal.pbio.0030079

Jellema, T., Baker, C. I., Wicker, B., \& Perrett, D. I. (2000). Neural representation for the perception of the intentionality of actions. Brain and Cognition, 44(2), 280-302. https://doi.org/10.1006/brcg.2000.1231

Jolij, J., \& Lamme, V. A. F. (2005). Repression of unconscious information by conscious processing: Evidence from affective blindsight induced by transcranial magnetic stimulation. Proceedings of the National Academy of Sciences, 102(30), 10747-10751. https://doi.org/10.1073/pnas.0500834102

Kana, R. K., \& Travers, B. G. (2012). Neural substrates of interpreting actions and emotions from body postures. Social Cognitive and Affective Neuroscience, 7(4), 446-56. 
https://doi.org/10.1093/scan/nsr022

Kitada, R., Johnsrude, I. S., Kochiyama, T., \& Lederman, S. J. (2010). Brain networks involved in haptic and visual identification of facial expressions of emotion: an fMRI study. NeuroImage, 49(2), 167789. https://doi.org/10.1016/j.neuroimage.2009.09.014

Kret, M. E., Pichon, S., Grèzes, J., \& de Gelder, B. (2011). Men fear other men most: Gender specific brain activations in perceiving threat from dynamic faces and bodies-an fMRI study. Frontiers in Psychology, 2(JAN), 1-11. https://doi.org/10.3389/fpsyg.2011.00003

Kret, M. E., Pichon, S., Grèzes, J., \& de Gelder, B. (2011). Similarities and differences in perceiving threat from dynamic faces and bodies. An fMRI study. NeuroImage, 54(2), 1755-62. https://doi.org/10.1016/j.neuroimage.2010.08.012

Lagravinese, G., Bisio, A., De Ferrari, A. R., Pelosin, E., Ruggeri, P., Bove, M., \& Avanzino, L. (2017). An Emotion-Enriched Context Influences the Effect of Action Observation on Cortical Excitability. Frontiers in Human Neuroscience, 11(October), 504. https://doi.org/10.3389/fnhum.2017.00504

Liddell, B. J., Brown, K. J., Kemp, A. H., Barton, M. J., Das, P., Peduto, A., ... Williams, L. M. (2005). A direct brainstem-amygdala-cortical "alarm" system for subliminal signals of fear. NeuroImage, 24(1), 235-243. https://doi.org/10.1016/j.neuroimage.2004.08.016

Lourenco, S. F., Longo, M. R., \& Pathman, T. (2011). Near space and its relation to claustrophobic fear. Cognition, 119(3), 448-453. https://doi.org/10.1016/j.cognition.2011.02.009

Makris, N., Kennedy, D. N., Mcinerney, S., Sorensen, A. G., Wang, R., Caviness, V. S., \& Pandya, D. N. (2005). Segmentation of Subcomponents within the Superior Longitudinal Fascicle in Humans : A Quantitative, In Vivo, DT-MRI Study. Cerebral Cortex, 15(6), 854-869. https://doi.org/10.1093/cercor/bhh186

Marchetti, C., \& Della Salla, S. (1998). Disentangling the Alien and Anarchic Hand. Cognitive Neuropsycbiatry, 3(3), 191-207. https://doi.org/10.1080/135468098396143

Mars, R. B., Jbabdi, S., Sallet, J., O’Reilly, J. X., Croxson, P. L., Olivier, E., ... Rushworth, M. F. S. (2011). Diffusion-Weighted Imaging Tractography-Based Parcellation of the Human Parietal Cortex and Comparison with Human and Macaque Resting-State Functional Connectivity. Journal of Neuroscience, 31(11), 4087-4100. https://doi.org/10.1523/JNEUROSCI.5102-10.2011

Mazzoni, N., Jacobs, C., Venuti, P., Silvanto, J., \& Cattaneo, L. (2017). State-dependent TMS reveals representation of affective body movements in the anterior intraparietal cortex. https://doi.org/10.1523/JNEUROSCI.0913-17.2017

Meeren, H. K. M., Hadjikhani, N., Ahlfors, S. P., Hämäläinen, M. S., \& de Gelder, B. (2016). Early Preferential Responses to Fear Stimuli in Human Right Dorsal Visual Stream - A MEG Study. Scientific Reports, 6(April), 24831. https://doi.org/10.1038/srep24831

Morris, J. S., de Gelder, B., Weiskrantz, L., \& Dolan, R. J. (2001). Differential extrageniculostriate and amygdala responses to presentation of emotional faces in a cortically blind field. Brain, 124(6), 1241-52. Retrieved from http://www.ncbi.nlm.nih.gov/pubmed/11353739

Morris, J. S., Ohman, A., \& Dolan, R. J. (1999). A subcortical pathway to the right amygdala mediating "unseen" fear. Proceedings of the National Academy of Sciences, 96(4), 1680-1685. 
https://doi.org/10.1073/pnas.96.4.1680

Pegna, A. J., Khateb, A., Lazeyras, F., \& Seghier, M. L. (2005). Discriminating emotional faces without primary visual cortices involves the right amygdala. Nature Neuroscience, 8(1), 24-25. https://doi.org/10.1038/nn1364

Pichon, S., de Gelder, B., \& Grezes, J. (2008). Emotional modulation of visual and motor areas by dynamic body expressions of anger. Social Neuroscience, 3(3-4), 199-212. https://doi.org/10.1080/17470910701394368

Pichon, S., de Gelder, B., \& Grèzes, J. (2009). Two different faces of threat. Comparing the neural systems for recognizing fear and anger in dynamic body expressions. NeuroImage, 47(4), 1873-1883. https://doi.org/10.1016/j.neuroimage.2009.03.084

Rizzolatti, G., Fogassi, L., \& Gallese, V. (2001). Neurophysiological mechanisms underlying the understanding and imitation of action. Nature Revienss Neuroscience, 2(9), 661-670. https://doi.org/10.1038/35090060

Rizzolatti, G., Scandolara, C., Matelli, M., \& Gentilucci, M. (1981). Afferent properties of periarcuate neurons in macaque monkeys. II. Visual responses. Behavioural Brain Research, 2(2), 147-163. https://doi.org/10.1016/0166-4328(81)90053-X

Robinson, D. L., \& Petersen, S. E. (1992). The pulvinar and visual salience. Trends in Neurosciences, 15(4), 127-132. https://doi.org/10.1016/0166-2236(92)90354-B

Roelofs, K. (2017). Freeze for action: neurobiological mechanisms in animal and human freezing. Philosophical Transactions of the Royal Society B: Biological Sciences, 372(1718), 20160206. https://doi.org/10.1098/rstb.2016.0206

Roelofs, K., Hagenaars, M. A., \& Stins, J. (2010). Facing freeze: social threat induces bodily freeze in humans. Psychological Science, 21(11), 1575-1581. https://doi.org/10.1177/0956797610384746

Rothwell, J. C. (1997). Techniques and mechanisms of action of transcranial stimulation of the human motor cortex. Journal of Neuroscience Methods, 74(2), 113-122. https://doi.org/10.1016/S01650270(97)02242-5

Ruiz-Padial, E., Mata, J. L., Rodríguez, S., Fernández, M. C., \& Vila, J. (2005). Non-conscious modulation of cardiac defense by masked phobic pictures. International Journal of Psychophysiology, 56(3), 271-281. https://doi.org/10.1016/j.ijpsycho.2004.12.010

Ruiz-Padial, E., Vila, J., \& Thayer, J. F. (2011). The effect of conscious and non-conscious presentation of biologically relevant emotion pictures on emotion modulated startle and phasic heart rate. International Journal of Psychophysiology, 79(3), 341-346. https://doi.org/10.1016/j.ijpsycho.2010.12.001

Sambo, C. F., Forster, B., Williams, S. C., \& Iannetti, G. D. (2012). To Blink or Not to Blink: Fine Cognitive Tuning of the Defensive Peripersonal Space. Journal of Neuroscience, 32(37), 12921-12927. https://doi.org/10.1523/JNEUROSCI.0607-12.2012

Sambo, C. F., \& Iannetti, G. D. (2013). Better Safe Than Sorry? The Safety Margin Surrounding the Body Is Increased by Anxiety. Journal of Neuroscience, 33(35), 14225-14230. https://doi.org/10.1523/JNEUROSCI.0706-13.2013

Sarkheil, P., Goebel, R., Schneider, F., \& Mathiak, K. (2013). Emotion unfolded by motion: a role for 
parietal lobe in decoding dynamic facial expressions. Social Cognitive and Affective Neuroscience, 8(8), 950-7. https://doi.org/10.1093/scan/nss092

Schenberg, L. C., Póvoa, R. M. F., Costa, A. L. P., Caldellas, A. V., Tufik, S., \& Bittencourt, A. S. (2005). Functional specializations within the tectum defense systems of the rat. Neuroscience and Biobehavioral Reviews, 29(8), 1279-1298. https://doi.org/10.1016/j.neubiorev.2005.05.006

Schutter, D. J. L. G., Hofman, D., \& Van Honk, J. (2008). Fearful faces selectively increase corticospinal motor tract excitability: A transcranial magnetic stimulation study. Psychophysiology, 45(3), 345-348. https://doi.org/10.1111/j.1469-8986.2007.00635.x

Tajadura-Jiménez, A., Pantelidou, G., Rebacz, P., Västfjäll, D., \& Tsakiris, M. (2011). I-space: The effects of emotional valence and source of music on interpersonal distance. PLOS ONE, 6(10). https://doi.org/10.1371/journal.pone.0026083

Tamietto, M., Castelli, L., Vighetti, S., Perozzo, P., Geminiani, G., Weiskrantz, L., \& de Gelder, B. (2009). Unseen facial and bodily expressions trigger fast emotional reactions. Proceedings of the National Academy of Sciences of the United States of America, 106(42), 17661-17666. https://doi.org/10.1073/pnas.0908994106

Tamietto, M., Cauda, F., Celeghin, A., Diano, M., Costa, T., Cossa, F. M., ... de Gelder, B. (2015). Once you feel it, you see it: Insula and sensory-motor contribution to visual awareness for fearful bodies in parietal neglect. Cortex, 62, 56-72. https://doi.org/10.1016/j.cortex.2014.10.009

Tamietto, M., \& de Gelder, B. (2008). Emotional contagion for unseen bodily expressions: evidence from facial emg. 2008 8th IEEE International Conference on Automatic Face and Gesture Recognition, FG 2008, 1-5. https://doi.org/10.1109/AFGR.2008.4813317

Tamietto, M., \& de Gelder, B. (2010). Neural bases of the non-conscious perception of emotional signals. Nature Reviews. Neuroscience, 11(10), 697-709. https://doi.org/10.1038/nrn2889

Tsakiris, M., Tajadura-Jiménez, A., \& Costantini, M. (2011). Just a heartbeat away from one's body: interoceptive sensitivity predicts malleability of body-representations. Proceedings. Biological Sciences / The Royal Society, 278(1717), 2470-2476. https://doi.org/10.1098/rspb.2010.2547

Vagnoni, E., Lourenco, S. F., \& Longo, M. R. (2012). Threat modulates perception of looming visual stimuli. Current Biology, 22(19), R826-R827. https://doi.org/10.1016/j.cub.2012.07.053

Vallar, G., \& Ronchi, R. (2009). Somatoparaphrenia: A body delusion. A review of the neuropsychological literature. Experimental Brain Research, 192(3), 533-551. https://doi.org/10.1007/s00221-008-1562y

Van den Stock, J., Tamietto, M., Sorger, B., Pichon, S., Grézes, J., \& de Gelder, B. (2011). Cortico-subcortical visual, somatosensory, and motor activations for perceiving dynamic whole-body emotional expressions with and without striate cortex (V1). Proceedings of the National Academy of Sciences of the United States of America, 108(39), 16188-93. https://doi.org/10.1073/pnas.1107214108

Van den Stock, J., Tamietto, M., Zhan, M., Heinecke, A., Hervais-Adelman, A., Legrand, L. B., ... de Gelder, B. (2014). Neural correlates of body and face perception following bilateral destruction of the primary visual cortices. Frontiers in Behavioral Neuroscience, 8(February), 1-10. https://doi.org/10.3389/fnbeh.2014.00030

Van Honk, J., Tuiten, A., Van Den Hout, M., Koppeschaar, H., Thijssen, J., De Haan, E., \& Verbaten, 
R. (1998). Baseline salivary cortisol levels and preconscious selective attention for threat: A pilot study. Psychoneuroendocrinology, 23(7), 741-747. https://doi.org/10.1016/S0306-4530(98)00047-X

van Loon, A. M., van den Wildenberg, W. P. M., van Stegeren, A. H., Ridderinkhof, K. R., \& Hajcak, G. (2010). Emotional stimuli modulate readiness for action: A transcranial magnetic stimulation study. Cognitive, Affective, \& Behavioral Neuroscience, 10(2), 174-181. https://doi.org/10.3758/CABN.10.2.174

Whalen, P. J., Kagan, J., Cook, R. G., Davis, F. C., Kim, H., Polis, S., .. Johnstone, T. (2004). Human amygdala responsivity to masked fearful eye whites. Science, 306(5704), 2061. https://doi.org/10.1126/science.1103617

Whalen, P. J., Rauch, S. L., Etcoff, N. L., McInerney, S. C., Lee, M. B., \& Jenike, M. A. (1998). Masked presentations of emotional facial expressions modulate amygdala activity without explicit knowledge. Journal of Neuroscience, 18(1), 411-418. https://doi.org/9412517

Williams, M. A. (2004). Amygdala Responses to Fearful and Happy Facial Expressions under Conditions of Binocular Suppression. Journal of Neuroscience, 24(12), 2898-2904. https://doi.org/10.1523/JNEUROSCI.4977-03.2004

Zhan, M., Hortensius, R., \& de Gelder, B. (2015). The body as a tool for anger awareness-differential effects of angry facial and bodily expressions on suppression from awareness. PLoS ONE, 10(10), 1-13. https://doi.org/10.1371/journal.pone.0139768 



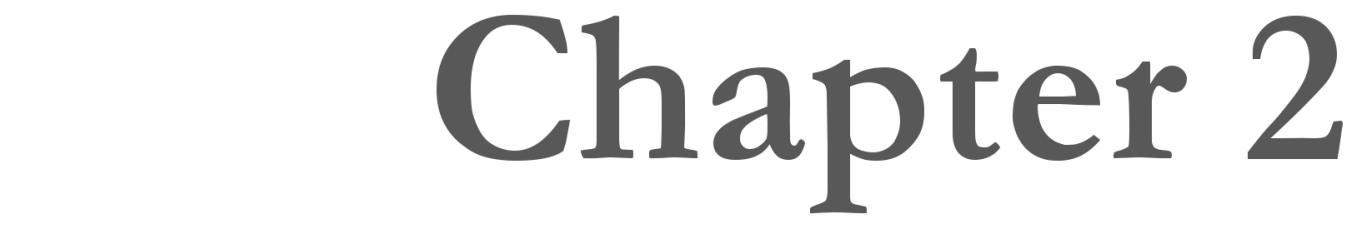

\section{A causal role for inferior parietal lobule in emotion body perception}

Based on:

Engelen, T., de Graaf, T. A., Sack, A. T., \& de Gelder, B. (2015). A causal role for inferior parietal lobule in emotion body perception. Cortex, 73, 195-202. 



\begin{abstract}
Recent investigations of emotion body perception have established that perceiving fearful body expressions critically triggers activity in dorsal stream structures related to action preparation. However, the causal contributions of these areas remain unclear. In the current experiment, we addressed this issue using online transcranial magnetic stimulation (TMS) of the inferior parietal lobule (IPL) in the dorsal stream and visual areas (extrastriate body area (EBA) in the ventral stream and early visual cortex (EVC)). Participants performed a delayed-match-to-sample task requiring detection of a change in posture of body expressions that were either neutral or fearful. Results revealed a significant interaction between the stimulation site and the emotional valence of stimuli, indicating that processing of emotional versus neutral bodies is affected differentially by stimulation of different central areas in body processing. IPL stimulation specifically enhanced fearful body processing. These findings relate emotion processing to separate processing streams, and moreover provide the first evidence that IPL plays a causal role in processing of fearful bodies.
\end{abstract}

\title{
Introduction
}

Some emotional body expressions that we see in people around us prompt us to flee, while others prompt us to fight. Recognizing bodily emotion expressions is obviously an important social ability and failure to process such expressions may make us miss important cues and have undesirable consequences. Emotional body expressions are complex stimuli because they convey simultaneously visual information about a body and cues about the emotion it expresses, as well as information about the action taking place (de Gelder, 2006). It is still an open question exactly how the information flows between the early visual cortex (EVC), sensitive to affective valence of the signal, visual areas in temporal cortex which encode object shape, and dorsal areas involved in action perception. In this paper we use multi-site TMS to identify the specific causal role of areas at different stages of visual processing, namely the early visual, temporal and parietal areas, during processing of neutral and fear bodily expressions. Consider the role of early cortical visual areas in the processing of emotional stimuli. A recent TMS experiment with healthy participants, in which single TMS pulses were given over V1 at phosphene location, showed an impairment of discrimination of neutral bodies. However, stimulation did not affect discrimination of threatening bodies (Filmer \& Monsell, 2013). This finding is consistent with evidence from patient studies showing that, despite destruction of primary visual cortex (V1), processing of emotions can still occur via a subcortical route to the amygdala, independent of the geniculo-striate pathway (de Gelder, Vroomen, Pourtois, \& Weiskrantz, 1999; Morris, de Gelder, Weiskrantz, \& Dolan, 2001; Tamietto, Pullens, de Gelder, Weiskrantz, \& Goebel, 2012; Van den Stock et al., 2011; 2014). Based on this we expect that stimulation of EVC will have a different effect depending on whether the stimuli are neutral or fearful.

A next question concerns the role of stimulus representation in ventral areas. Some investigations of neutral body images have mainly focused on the role of a category specific area in the temporal cortex, dedicated to body perception, the extrastriate body area (EBA) (Downing, Jiang, Shuman, \& Kanwisher, 2001). By now the role of EBA in processing of neutral body images has been established by many studies (Kret, Pichon, Grèzes, \& de Gelder, 2011; Pitcher, Charles, Devlin, Walsh, \& Duchaine, 2009; Pitcher, Goldhaber, Duchaine, Walsh, \& Kanwisher, 2012; Urgesi, Berlucchi, \& Aglioti, 2004). But it is still unclear whether body 
category representation in EBA is an essential stage in emotion processing (de Gelder, 2016). Previously found activations in EBA in response to emotion bodies may be driven by attention and arousal effects (Downing \& Peelen, 2011). Given the role of EBA in body processing, we predict that TMS stimulation of this area should selectively affect the processing of neutral bodies.

In contrast to ventral processing structures, recent studies have discovered an important role for the parietal cortex in emotional action obstervation (de Gelder, Snyder, Greve, Gerard, \& Hadjikhani, 2004; Goldberg, Christensen, Flash, Giese, \& Malach, 2015; Grèzes, Pichon, \& de Gelder, 2007; Pichon, de Gelder, \& Grezes, 2008). Consistent with this, Goldberg, Preminger, \& Malach (2014) recently provided fMRI evidence for this emotion-action link by showing that dynamic emotional whole-body stimuli preferentially activate parietal areas, traditionally associated with the mirror neuron network. Furthermore, confrontation with emotional bodies leads to an increase in cortical excitability in primary motor cortex (Borgomaneri, Gazzola, \& Avenanti, 2012, 2014). Thus, seeing emotional bodies may involve dorsal parietal structures and prime motor responses more than seeing neutral bodies (Borgomaneri et al., 2012; de Gelder et al., 2004).

The goal of the current study was to disentangle the causal roles of the inferior parietal lobule (IPL) in the dorsal stream, EBA in the ventral stream and early visual cortex (EVC) during visual processing of neutral and fearful bodies using online triple pulse TMS (tpTMS). IPL is a hub in the dorsal action stream that is strongly connected with premotor and motor areas as well as with subcortical areas (Clower, West, Lynch, \& Strick, 2001), and is thus hypothesized as a key structure in emotion body perception. Given the evidence for the link between parietal dorsal stream areas and processing of emotion bodies, we predict that stimulation of IPL should selectively alter performance in a fear body condition as compared to a neutral body condition.

\section{Methods}

\section{Participants}

Twenty healthy volunteers (11 female, mean age $(\mathrm{SD})=26(3,5))$ participated in the TMS experiment. Eighteen were right handed and all had normal or corrected to normal vision. Participants were unaware of the goal of the study until after the completion of the experiment. Participants were screened for fMRI and TMS safety. The study was performed in accordance to the Declaration of Helsinki and approved by the local ethical committee. Two participants were excluded from analysis because of missing data.

\section{Brain imaging}

We determined the location of EBA by using fMRI guided neuro-navigation, because this method has been shown to be superior compared to using group functional Talairach coordinates (Sack et al., 2009). Each participant took part in an fMRI localizer experiment, in which five different visual stimulus categories were presented. Static images of each condition (bodies, faces, houses, tools and words) were shown pseudo-randomized in a blocked design, with 7 blocks per condition. Functional images were acquired using a 3T MAGNETOM Prisma fit scanner with a 64-channel head-neck coil. A gradient-echo EPI sequence (repetition time (TR) $=2 \mathrm{~s}$, echo time $(\mathrm{TE})=31 \mathrm{~ms}$, voxel size $=2 \times 2 \times 2 \mathrm{~mm}$, 64 slices $)$ providing whole-brain 
coverage was used. The localizer scan started with a fixation period of $6 \mathrm{TRs}$, followed by blocks of stimuli of 6 TRs separated by fixation periods of 6TRs, and ending with a fixation period of 12 TRs. A total of 432 volumes were acquired. We defined right EBA by contrasting the fMRI responses to bodies and houses and selecting the cluster that showed greatest relative activation for the bodies' condition.

A high-resolution T1-weighted MPRAGE anatomical scan was performed to determine the two other stimulation sites based on individual brain anatomy (EVC and IPL). Without access to a straightforward localizer as was available for EBA, and considering that individual MRIguided TMS localization can lead to adequate effect sizes (Sack et al., 2009), stimulation locations for IPL and EVC were determined based on MRI landmarks. For 'IPL', we targeted a location directly underneath IPS; either the end of the upper branch of superior temporal sulcus or the end of the posterior branch of the Sylvian Fissure, depending on individual anatomy (see Cattaneo, Sandrini, \& Schwarzbach, 2010). For EVC we targeted the spot between the calcarine sulci of both hemispheres. Functional and anatomical brain imaging data were preprocessed and analyzed offline using BrainVoyager QX (Brain Innovation BV, Maastricht, The Netherlands). All three target locations are visualized on the template brain shown in Figure 1(b) and average Talairach coordinates of all stimulation sites are reported in table 1.

A

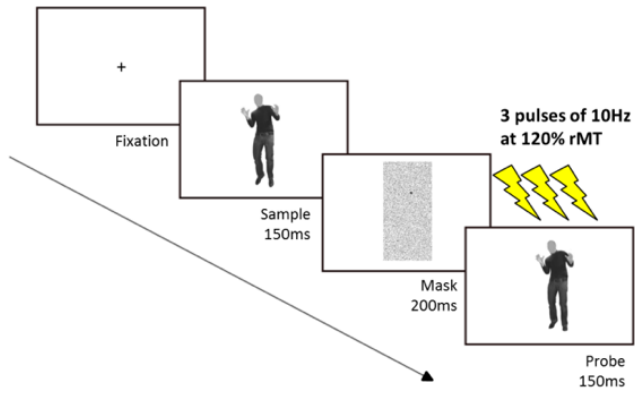

B

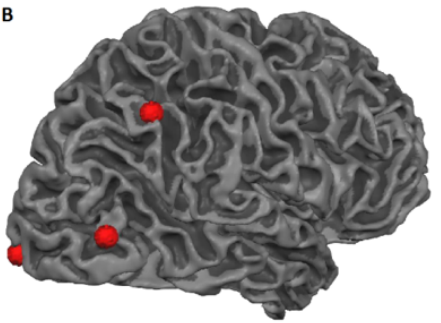

Figure 1. An example of a timeline of the experimental trial procedure (a) and TMS Target sites (b)

Table 1. Average Talairach coordinates per stimulation site \pm standard deviation

\begin{tabular}{cccr}
\hline Talairach coordinates & EBA & IPL & EVC \\
\hline$x$ & $47 \pm 3$ & $42 \pm 6$ & $0 \pm 3$ \\
$y$ & $-72 \pm 6$ & $-54 \pm 4$ & $-90 \pm 1$ \\
$z$ & $1 \pm 5$ & $48 \pm 4$ & $-7 \pm 5$ \\
\hline
\end{tabular}




\section{Stimuli}

Body stimuli were selected from a set of dynamic video clips showing the frontal view of four male actors jumping backwards with their hands forward (fearful body) or swinging their arms along the side of their trunk (neutral body). Two different frames were selected per actor for each condition. All faces were covered with a gray mask. The bodies were presented centrally on a gray background using a LCD monitor (resolution, $1920 \times 1080$ ) at an eye-screen distance of $\sim 57 \mathrm{~cm}$.

Task

A similar delayed-match-to sample task was used as described in Candidi, Stienen, Aglioti, \& de Gelder (2011), in which participants had to judge whether a sample stimulus matched a probe. Each trial showed either neutral or fearful bodies and the identity of the body was kept constant within a trial. The sample stimulus was presented for $150 \mathrm{~ms}$, followed by the presentation of a $200 \mathrm{~ms}$ mask, after which the probe was shown for $150 \mathrm{~ms}$ (see figure 1 (a) for an example trial). After the presentation of the probe, the participants had to give a response. The inter-trial interval was jittered around 7 seconds. The Presentation software package (NeuroBehavioural Systems, Albany, CA) was used for stimulus presentation, recording of responses and triggering of the TMS pulses.

\section{TMS stimulation and site localization}

During the performance of the task, online triplets of TMS pulses with a $100 \mathrm{~ms}$ interval were delivered at $120 \%$ resting motor threshold (mean stimulation intensity $39 \%$ MSO) using a MCB70 figure-of-eight coil and Magpro X100 stimulator (Medtronic Functional Diagnostics A/S, Skovlunde, Denmark). Onset of TMS pulses coincided with the appearance of the probe stimulus, so that the period of stimulation overlapped with previously found critical points of body processing for each of the stimulation sites: EBA at 100-110ms (Pitcher et al., 2012), V1 70140ms (Filmer \& Monsell, 2013) and IPL 80-110ms post stimulus onset (Meeren, Hadjikani, Ahlfors, Hämäläinen, \& de Gelder, 2014). For the stimulation of IPL, the coil was positioned with the handle pointing backward and outward at a $45^{\circ}$ angle from the mid- sagittal axis. For the stimulation of EBA, the coil was positioned with the handle pointing backward and inward and medially at a $45^{\circ}$ angle from the mid-sagittal axis. During the stimulation of bilateral EVC, the coil was positioned with the handle pointing upward aligned with the mid-sagittal axis. During the sham stimulation the coil was positioned roughly in-between the other three stimulation sites, with the coil tilted so that no real stimulation was applied. For each stimulation condition the coil was fixed in a coil holder for the entire duration of the run.

\section{Procedure}

Participants came in for a two-hour TMS session in which all four stimulation sites were tested. First, a 32 trial practice of the delayed-match-to-sample task was performed to ensure above chance level performance. After the participant was comfortable with the task, motor threshold was established by moving the coil over primary motor cortex until an optimal position was found for eliciting muscle twitches in the hand muscles. After this, the stimulation intensity was decreased until a threshold was found at which 5 out of 10 pulses still evoked a motor response at rest. Next, the correct coil location on the scalp was determined for each 
stimulation site by using the BrainVoyager TMS Neuronavigator system (Brain Innovation, Maastricht, the Netherlands). The anatomical and functional data acquired during the fMRI session were imported into the Neuronavigation software and used for stereotaxic co-registration of the participant's brain with the TMS coil, allowing for online coil positioning. For each stimulation site the participant performed 64 trials of the task (32 fear trials and 32 neutral trials). The order of stimulation sites and sham stimulation were randomized for each participant.

\section{Analysis}

The dependent variable in this experiment is accuracy in detecting a postural change between sample and probe. As a measure of how sensitive the participant was to detection of the signal from the noise, we calculated $d$ ', an unbiased measure of performance accuracy. $D$ 'is normally calculated by subtracting transformed hit $(\mathrm{H})$ and false alarm rates (FA). A $d$ ' of 0 reflects the inability of the participant to correctly identify postural change. To correct for ceiling effects, a corrected hit rate $\left(\mathrm{H}^{\prime}\right)$ and false alarm rate $\left(\mathrm{FA}^{\prime}\right)$ were calculated, as proposed by Snodgrass \& Corwin (1988), using the following formulas:

$$
\begin{aligned}
\mathrm{H}^{\prime} & =(\mathrm{h}+0.5) /(\mathrm{h}+\mathrm{m}+1) \\
\mathrm{FA}^{\prime} & =(\mathrm{fa}+0.5) /(\mathrm{fa}+\mathrm{cr}+1)
\end{aligned}
$$

where $\mathrm{h}$ is the number of hits, $\mathrm{m}$ is the number of misses, $\mathrm{fa}$ is the number of false alarms and cr is the number of correct rejections (Candidi et al., 2011; Snodgrass \& Corwin, 1988; Tamietto, Geminiani, Genero, \& de Gelder, 2007).

To ensure that possible differences between the neutral and fear condition did not affect direct comparisons within a stimulation site, difference scores were calculated by subtracting individual $d$ ' sham scores per condition (fear/neutral) from each individual $d$ ' score per stimulation site and condition.

A repeated measures ANOVA was performed on difference scores with condition (fear/neutral) and stimulation site (EBA/IPL/EVC) as within subject factors. Before calculation of $d$, outliers were removed based on reaction times that deviated more than 2.5 times the standard deviation from the mean within a subject per stimulation site and condition. Multivariate results (Pillai's trace) are reported.

It is important to control for possible differences in implied motion between the neutral and fear condition. To this end, a control experiment was run in an independent sample of 10 participants. They performed 64 trials of the task with the same parameters as the delayedmatch-to-sample task, only they were asked to express in millimeters the difference in implied movement they perceived between the sample and the probe (see figure 2). Results indicated that there was no significant difference of implied motion between the neutral and fear condition $(p=0.673)$, but there was a significant difference between same and different stimuli $(p=$ 0.031). Thus, participants subjectively noticed the change in posture, but importantly did not perceive more motion for fearful stimuli as compared to neutral stimuli. 


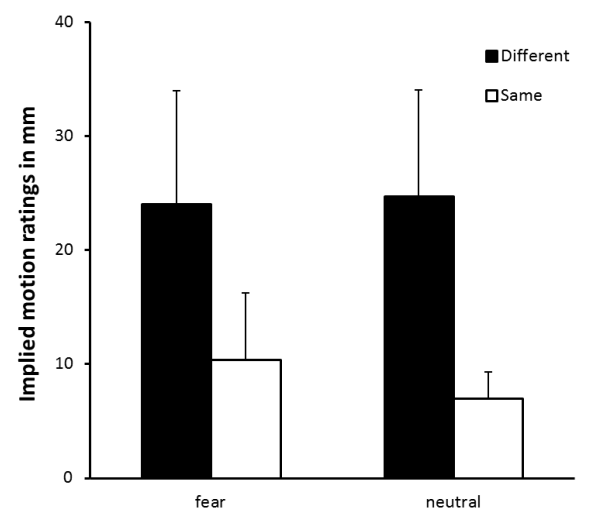

Figure 2. Implied motion measured in a separate sample of participants showed that the subjective experience of motion did not differ significantly between the fear and neutral condition.

To ensure that neutral and fear stimuli were identified as such, a validation experiment was performed in the same sample of participants from the control experiment just described. Each stimulus was presented for $150 \mathrm{~ms}$ and the participant had to indicate whether they thought the body posture was in a neutral or fearful posture. Average accuracy for the fear bodies was $96 \%$ and for the neutral bodies accuracy was $91 \%$.

To rule out any influences of speed-accuracy trade off effects, an analysis on reaction times (RTs) was performed (see table 2 for average RTs per condition). Results showed that there were no main effects of condition $(V=0.023, F(1,17)=0.402, p=0.534)$, stimulation site $(V$ $=0.212, F(3,15)=1.348, p=0.296)$, nor a significant interaction $(V=0.259, F(3,15)=1.747$, $p=0.2)$.

To see if TMS had any effects on response bias, criterion values were calculated by multiplying the sum of H' and FA' by -0.5 . Results of the analysis of the criterion values showed a main effect for condition $(V=.701, \mathrm{~F}(1,17)=39.767, p<.00)$, reflecting a bias to answering 'same' in the fear condition. There was no main effect of stimulation site $(V=.034, \mathrm{~F}(3,15)=.174$, $p=.912)$, nor a condition $\mathrm{x}$ stimulation site interaction $(V=.235, \mathrm{~F}(3,15)=1.533, p=.247)$, showing that TMS stimulation did not alter response bias.

A preliminary analysis was performed on raw ' $d$ scores. Results of this analysis showed there was no main effect for condition $(V=0.052, \mathrm{~F}(1,17)=0.938, p=0.346)$. There was, however, a main effect for stimulation site $(V=0.464, \mathrm{~F}(3,15)=4.334, p=0.022)$, as well as a significant condition $\mathrm{x}$ stimulation site interaction $(V=0.502, \mathrm{~F}(3,15)=5.046, p=0.013)$. Analysis of the sham conditions showed that there was no significant difference between the two conditions $(V=0.053, \mathrm{~F}(1,17)=0.946, p=0.344)$, ruling out any possible differences in task difficulty between neutral and fear. 


\section{Results}

In this experiment, participants performed a delayed match-to-sample tasks on neutral versus fearful body postures, while receiving online TMS over either the inferior parietal lobule (IPL), the extrastriate body area (EBA), the early visual cortex (EVC), or SHAM TMS. Results indicated a significant main effect for stimulation site $(V=0.448, F(2,16)=6.492, p=0.009)$, as well as a significant condition (fear/neutral) $\mathrm{x}$ stimulation site (IPL/EBA/EVC) interaction ( $V$ $=0.472, F(2,16)=7.152, p=0.006)$.

Based on the significant interaction effect, data were first split per stimulation site (EBA/IPL/EVC), to see how fear and neutral bodies differentially affect one of the stimulation sites. There was no significant main effect of condition in EBA $(V=0.077, F=1,17)=$ $1.421, p=0.25)$, IPL $(V=0.083, F=1,17)=1.547, p=0.231)$ or $\operatorname{EVC}(V=0.068, F=1$, 17) $=1.242, p=0.281)$.

To further disentangle the significant interaction effect, data were then split per condition (fear/neutral), to see how emotion can affect different nodes within the body processing network. The results of the analysis looking only at the fear condition showed that a significant main effect of stimulation site, $V=0.563, F(2,16)=10.298, p=0.001$. Bonferroni corrected post-hoc pairwise comparisons showed a significant differential effect of stimulation of EBA and IPL in the fear body condition $(p=0.001)$, but no such effect was apparent for comparisons between EBA and EVC $(p=0.67)$, or IPL and EVC $(p=0.133)$. To check for the direction of the effect of EBA and IPL in the fear body condition, one sampled t-tests were performed for EBA and IPL. The mean of EBA in the fear body condition did not significantly differ from $0,(t(17)=-1.018, p=0.165)$, whereas for IPL there was a significant effect $(t(17)$ $=2.6, p=0.0095)$.

A repeated measures ANOVA, looking only at the neutral condition, showed a significant main effect of stimulation site, $V=0.323, F(2,16)=3.818, p=0.044$. Bonferroni corrected posthoc pairwise comparisons showed a significant differential effect of stimulation of IPL and $\operatorname{EVC}(p=0.035)$, but this effect was absent in the comparison between IPL and EBA $(p=1)$, or EBA and EVC $(p=0.351)$. One sample t-tests did not show a significant increase or decrease from 0 for either IPL $(t(17)=0.663, \mathrm{p}=0.258)$ or $\operatorname{EVC}(t(17)=-1.244, p=0.115)$. Statistical results of analysis of the difference scores are visualized in figure 3 .

These results demonstrate that the IPL is causally engaged in emotion body processing, whereas the selective disruption of neutral body processing after EVC stimulation demonstrates that emotional information from fear bodies can still be processed independent of EVC.

Table 2. Raw $d$ and reaction time means \pm standard error

\begin{tabular}{llllll}
\hline & & EBA & IPL & EVC & sham \\
\hline$d^{\prime}$ & Fear & $0.84 \pm 0.13$ & $1.30 \pm 0.15$ & $1.01 \pm 0.15$ & $0.98 \pm 0.18$ \\
& Neutral & $1.20 \pm 0.11$ & $1.26 \pm 0.13$ & $0.95 \pm 0.16$ & $1.17 \pm 0.14$ \\
\multirow{2}{*}{ RTs (ms) } & Fear & $950 \pm 64$ & $895 \pm 54$ & $903 \pm 55$ & $920 \pm 53$ \\
& Neutral & $934 \pm 61$ & $896 \pm 60$ & $916 \pm 57$ & $888 \pm 51$ \\
\hline
\end{tabular}




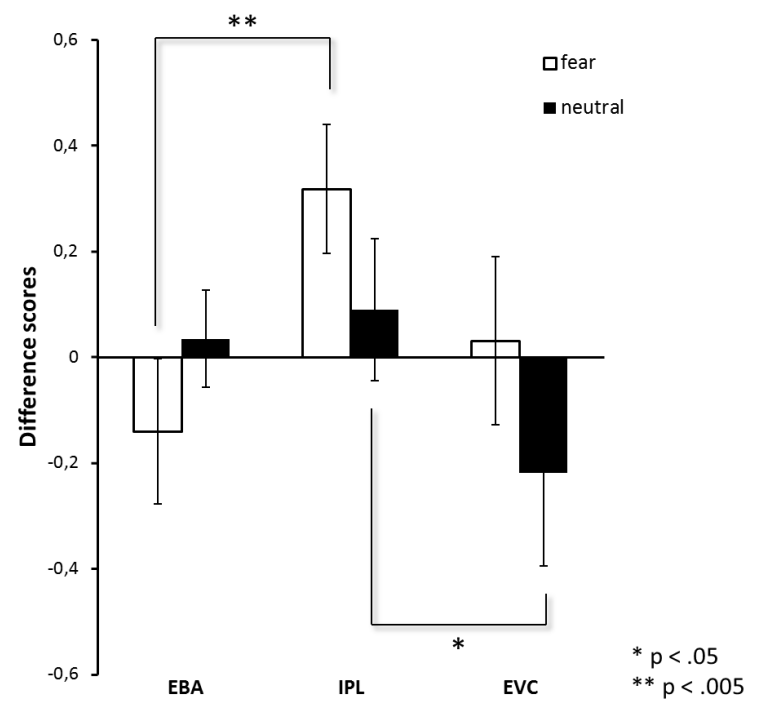

Figure 3. In the fear body condition, stimulation of IPL resulted in increased accuracy, as expressed in difference scores to SHAM, compared to stimulation of EBA. In the neutral body condition, stimulation of EVC resulted in a decrease of accuracy.

\section{Discussion}

The goal of the current study was to dissociate the causal roles of dorsal stream (IPL), ventral stream (EBA) and early visual cortex (EVC) using online TMS, while participants had to detect subtle posture changes of bodies that either expressed fear, or were in a neutral position. Given the importance of efficient recognition and appropriate response to bodies conveying emotions, our hypothesis was that body emotion expressions are processed in the dorsal action stream, as assumed in the separate pathway models (de Gelder \& Hadjikhani, 2006; Rudrauf et al., 2008; Tamietto \& de Gelder, 2010; Zhan, Hortensius, \& Gelder, 2015). Therefore, in contrast to emotional bodies, perception of neutral bodies that do not necessitate action related processing, is more likely to engage the ventral processing stream. Perception of emotional content should thus not be affected by disruption of areas involved in perception of neutral bodies.

Consistent with this, we found that stimulation of IPL led to a significant increase in task accuracy compared to the condition in which EBA was stimulated, and importantly this effect was specific to the fear body condition. In direct contrast, stimulation of EVC led to decreased performance compared to IPL, specifically for the neutral body condition, while performance in the fear body condition remained unaffected. 


\section{The role of IPL in the processing of fearful bodies}

As hypothesized, the observed TMS effect in IPL was specific to the fear condition. This critical role of IPL in emotion body processing is consistent with the literature on the functions of the parietal cortex. Neuroimaging experiments suggest the involvement of the parietal dorsal stream in the processing of emotions. It has, for example, been shown that both haptic and visual identification of the facial expression of emotions leads to activations in IPL (Kitada, Johnsrude, Kochiyama, \& Lederman, 2010). Similarly, IPL activation was found for the rating of dynamic expressive faces versus rating of gender (Sarkheil, Goebel, Schneider, \& Mathiak, 2013). Such findings have been extended by looking at activations specific for bodies expressing emotions. Kana \& Travers (2012) found that in female participants the right IPL was significantly more activated for emotional bodies versus baseline, whereas male participants showed greater activation in superior parietal lobule (SPL) while judging emotional bodies. Consistent with this idea, a recent MEG study has found early parietal activations in response to fearful bodies (Meeren et al., 2014). They directly compared perception of fearful and neutral bodies and found a significant cluster in right parietal cortex at $80-110 \mathrm{~ms}$ post stimulus onset.

Previously, Borgomaneri et al. (2012) showed that when neutral and emotional bodies were matched for implied motion, there was no difference between these two categories of stimuli in terms of their effects on motor cortex excitability. These findings raise the question whether with regard to the triggering of motor facilitation, it could rather be the implied motion that leads to previously observed effects of valence on motor excitability rather than emotional bodies by themselves (Borgomaneri et al., 2014; Hajcak et al., 2007; van Loon, van den Wildenberg, van Stegeren, Hajcak, \& Ridderinkhof, 2010). In the current experiment, implied motion was equal between neutral and fearful body conditions, and therefore the observed effect of IPL stimulation cannot be attributed to differences in implied motion alone.

Although we had no a priori expectation regarding the direction of TMS-induced behavioral changes, TMS is traditionally viewed as a virtual lesion approach, suggesting the expectation of a condition-and site-specific behavioral impairment. In contrast, we report here a conditionand site-specific behavioral improvement. For the empirical testing of the functional relevance of a given area the direction of the induced behavioral change is irrelevant. While the exact workings of TMS are far from understood, it has been suggested that whether performance on a task is facilitated or inhibited by TMS depends on the baseline activity and thus, state of that specific area (Silvanto, Cattaneo, Battelli, \& Pascual-Leone, 2008).

The current experiment has now provided causal evidence about the involvement of parietal cortex, and specifically IPL, in emotion body perception.

\section{Involvement of early visual cortex in neutral body processing}

Post-hoc tests looking at the neutral condition revealed a main effect for stimulation site, and the biggest modulation across sites was a decrease in performance with TMS over EVC. This suggests that TMS impaired neutral body processing when EVC was stimulated. However, since difference scores of EVC in the neutral condition did not significantly differ from 0 , this observation should be regarded with caution.

A specific effect of EVC in the neutral condition would be in line with the experiment by Filmer \& Monsell (2013). Their study used a TMS masking paradigm (for recent review see de 
Graaf, Koivisto, Jacobs, \& Sack (2014)) and showed that TMS over V1 led to a selective impairment on categorical discrimination of neutral, but not threatening bodies. These results are in accord with the idea that geniculo-striate pathway is not essential for emotion recognition and that emotional information could still be accessed via a subcortical route via amygdala (LeDoux (1996), Tamietto et al. (2012)). Previous studies have explored the implications of V1 lesions on emotion processing. These studies have found that despite the lack of conscious awareness of a stimulus, information about the emotional state of the presented stimulus is still processed to a certain extent (de Gelder \& Hadjikhani, 2006; de Gelder et al., 1999; Morris et al., 2001).

The results of EVC stimulation in the current experiment again hint at how emotion processing is possible independent of the genicolu-striate pathway, and are in direct contrast with the findings of stimulation of IPL, which selectively affected emotion body processing. This dissociation between EVC and IPL shows how emotion bodies can be processed in a specific non-geniculo-striate pathway, that can quickly relay emotion information to motor areas involved in action preparation. One possibility is that the information about an emotional body travels from superior colliculus directly to IPL (Clower et al., 2001). Based on current results, however, the possible involvement of extrastriate areas cannot be ruled out.

\section{Processing of neutral bodies in the ventral visual stream}

In the current experiment, we did not find a significant change in performance in either the fearful or neutral body condition during stimulation of EBA. From the large subject sample and reliable localization procedure, this null result has some credibility (de Graaf \& Sack, 2011) Moreover it is consistent with other TMS studies using emotional body expressions (Candidi et al., 2011). In contrast, earlier studies using only neutral bodies showed that EBA played a role in body processing, as stimulation of EBA led to disrupted performance in a delayedmatch-to-sample task (Pitcher et al., 2009, 2012; Urgesi et al., 2004; Urgesi, Calvo-Merino, Haggard, \& Aglioti, 2007). However, Urgesi et al. (2007) showed that in a delayed-match-tosample task, rTMS to EBA only interfered with processing of inverted, and not upright bodies, suggesting that rather than whole body units, only processing of individual body parts occurs in EBA. Likewise, Urgesi, Candidi, Ionta, \& Aglioti (2007) found that stimulation of EBA only interfered with morphological features of human bodies and not observed whole body actions. This could explain why in the current study no results were found after EBA stimulation, and is also in line with lesion studies showing deficits in processing of body parts, but not body actions (Moro et al., 2008).

The role of EBA in emotion processing is still unclear. Some neuroimaging studies have found activations in EBA in response to emotion body stimuli (e.g. Kret et al., 2011). However a review by Downing \& Peelen (2011) suggests that, rather than playing a role in emotion processing, previously found activations for emotion bodies are probably driven by attention and arousal rather than valence. 


\section{Conclusion}

Taken together, our results indicate that IPL is a central component in the processing route of emotion body expressions, consistent with the notion that seeing emotion body expression triggers action structures. This emotion processing takes place relatively independent of the visual representation of the body. Furthermore, this information may reach the parietal cortex via subcortical to extrastriate areas, independent of early visual areas. 


\section{References}

Borgomaneri, S., Gazzola, V., \& Avenanti, A. (2012). Motor mapping of implied actions during perception of emotional body language. Brain Stimulation, 5(2), 70-6. doi:10.1016/j.brs.2012.03.011

Borgomaneri, S., Gazzola, V., \& Avenanti, A. (2014). Transcranial magnetic stimulation reveals two functionally distinct stages of motor cortex involvement during perception of emotional body language. Brain Structure \& Function. doi:10.1007/s00429-014-0825-6

Candidi, M., Stienen, B. M. C., Aglioti, S. M., \& de Gelder, B. (2011). Event-Related Repetitive Transcranial Magnetic Stimulation of Posterior Superior Temporal Sulcus Improves the Detection of Threatening Postural Changes in Human Bodies. Journal of Neuroscience, 31(48), 17547-17554. doi:10.1523/JNEUROSCI.0697-11.2011

Cattaneo, L., Sandrini, M., \& Schwarzbach, J. (2010). State-dependent TMS reveals a hierarchical representation of observed acts in the temporal, parietal, and premotor cortices. Cerebral Cortex, 20(9), 2252-8. doi:10.1093/cercor/bhp291

Clower, D. M., West, R. a, Lynch, J. C., \& Strick, P. L. (2001). The inferior parietal lobule is the target of output from the superior colliculus, hippocampus, and cerebellum. The Journal of Neuroscience, 21(16), 6283-6291. doi:21/16/6283 [pii]

de Gelder, B. (2006). Towards the neurobiology of emotional body language. Nature Reviews. Neuroscience, 7(3), 242-9. doi:10.1038/nrn1872

de Gelder, B. (2016). Emotional Body Perception in the Wild. New York, NY: The Guilford Press

de Gelder, B., \& Hadjikhani, N. (2006). Non-conscious recognition of emotional body language. NeuroReport, 17(6), 583-586. doi:10.1097/00001756-200604240-00006

de Gelder, B., Snyder, J., Greve, D., Gerard, G., \& Hadjikhani, N. (2004). Fear fosters flight: a mechanism for fear contagion when perceiving emotion expressed by a whole body. Proceedings of the National Academy of Sciences of the United States of America, 101(47), 16701-6. doi:10.1073/pnas.0407042101

de Gelder, B., Vroomen, J., Pourtois, G., \& Weiskrantz, L. (1999). Non-conscious recognition of affect in the absence of striate cortex. NeuroReport, 10(18), 3759-3763. doi:10.1097/00001756199912160-00007

de Graaf, T. a., Koivisto, M., Jacobs, C., \& Sack, A. T. (2014). The chronometry of visual perception: Review of occipital TMS masking studies. Neuroscience and Biobehavioral Reviews, 45, 295-304. doi:10.1016/j.neubiorev.2014.06.017

de Graaf, T. A., \& Sack, A. T. (2011). Null results in TMS: From absence of evidence to evidence of absence. Neuroscience and Biobehavioral Reviews, 35, 871-877. doi:10.1016/j.neubiorev.2010.10.006

Downing, P. E., Jiang, Y., Shuman, M., \& Kanwisher, N. (2001). A cortical area selective for visual processing of the human body. Science, 293(5539), 2470-3. doi:10.1126/science.1063414 
Downing, P., \& Peelen, M. (2011). The role of occipitotemporal body-selective regions in person perception. Cognitive Neuroscience, 2, 186-226.

Filmer, H. L., \& Monsell, S. (2013). TMS to V1 spares discrimination of emotive relative to neutral body postures. Neuropsychologia, 51(13), 2485-91. doi:10.1016/j.neuropsychologia.2013.09.029

Goldberg, H., Christensen, A., Flash, T., Giese, M., \& Malach, R. (2015). Brain activity correlates with emotional perception induced by dynamic avatars. NeuroImage. doi:10.1016/j.neuroimage.2015.07.056

Goldberg, H., Preminger, S., \& Malach, R. (2014). The emotion-action link? Naturalistic emotional stimuli preferentially activate the human dorsal visual stream. Neurolmage, 84, 254-64. doi:10.1016/j.neuroimage.2013.08.032

Grèzes, J., Pichon, S., \& de Gelder, B. (2007). Perceiving fear in dynamic body expressions. NeuroImage, 35(2), 959-67. doi:10.1016/j.neuroimage.2006.11.030

Hajcak, G., Molnar, C., George, M. S., Bolger, K., Koola, J., \& Nahas, Z. (2007). Emotion facilitates action: a transcranial magnetic stimulation study of motor cortex excitability during picture viewing. Psychophysiology, 44(1), 91-7. doi:10.1111/j.1469-8986.2006.00487.x

Kana, R. K., \& Travers, B. G. (2012). Neural substrates of interpreting actions and emotions from body postures. Social Cognitive and Affective Neuroscience, 7(4), 446-56. doi:10.1093/scan/nsr022

Kitada, R., Johnsrude, I. S., Kochiyama, T., \& Lederman, S. J. (2010). Brain networks involved in haptic and visual identification of facial expressions of emotion: an fMRI study. NeuroImage, 49(2), 1677 89. doi:10.1016/j.neuroimage.2009.09.014

Kret, M. E., Pichon, S., Grèzes, J., \& de Gelder, B. (2011). Similarities and differences in perceiving threat from dynamic faces and bodies. An fMRI study. Neurolmage, 54(2), 1755-62. doi:10.1016/j.neuroimage.2010.08.012

LeDoux, J. E. (1996). The emotional brain. New York: Simon \& Schuster.

Meeren, H. K. M., Hadjikani, N., Ahlfors, S. P., Hämäläinen, M. S., \& de Gelder, B. (2014). Human right parietal cortex shows early preferential responses to naturalistic emotional stimuli: an MEG study. Poster presented at Society for Neuroscience conference. Washington DC, United States.

Moro, V., Urgesi, C., Pernigo, S., Lanteri, P., Pazzaglia, M., \& Aglioti, S. M. (2008). The Neural Basis of Body Form and Body Action Agnosia. Neuron, 60(2), 235-246. doi:10.1016/j.neuron.2008.09.022

Morris, J. S., de Gelder, B., Weiskrantz, L., \& Dolan, R. J. (2001). Differential extrageniculostriate and amygdala responses to presentation of emotional faces in a cortically blind field. Brain, 124(6), $1241-52$.

Pichon, S., de Gelder, B., \& Grezes, J. (2008). Emotional modulation of visual and motor areas by dynamic body expressions of anger. Social Neuroscience, 3(3-4), 199-212. doi:10.1080/17470910701394368 
Pitcher, D., Charles, L., Devlin, J. T., Walsh, V., \& Duchaine, B. (2009). Triple dissociation of faces, bodies, and objects in extrastriate cortex. Current Biology, 19(4), 319-24. doi:10.1016/j.cub.2009.01.007

Pitcher, D., Goldhaber, T., Duchaine, B., Walsh, V., \& Kanwisher, N. (2012). Two critical and functionally distinct stages of face and body perception. The Journal of Neuroscience, 32(45), $15877-$ 85. doi:10.1523/JNEUROSCI.2624-12.2012

Rudrauf, D., David, O., Lachaux, J.-P., Kovach, C. K., Martinerie, J., Renault, B., \& Damasio, A. (2008). Rapid interactions between the ventral visual stream and emotion-related structures rely on a twopathway architecture. The Journal of Neuroscience, 28(11), 2793-2803. doi:10.1523/JNEUROSCI.3476-07.2008

Sack, A. T., Kadosh, R. C., Schuhmann, T., Moerel, M., Walsh, V., \& Goebel, R. (2009). Optimizing Functional Accuracy of TMS in Cognitive Studies : A Comparison of Methods. Journal of Cognitive Neuroscience, 21(2), 207-221.

Sarkheil, P., Goebel, R., Schneider, F., \& Mathiak, K. (2013). Emotion unfolded by motion: a role for parietal lobe in decoding dynamic facial expressions. Social Cognitive and Affective Neuroscience, 8(8), 950-7. doi:10.1093/scan/nss092

Silvanto, J., Cattaneo, Z., Battelli, L., \& Pascual-Leone, A. (2008). Baseline cortical excitability determines whether TMS disrupts or facilitates behavior. Journal of Neurophysiology, 99(5), 2725-30. doi:10.1152/jn.01392.2007

Snodgrass, J. G., \& Corwin, J. (1988). Pragmatics of measuring recognition memory: Applications to dementia and amnesia. Journal of Experimental Psychology: General, 117(1), 34-50. doi:10.1037//00963445.117.1.34

Tamietto, M., \& de Gelder, B. (2010). Neural bases of the non-conscious perception of emotional signals. Nature Reviews. Neuroscience, 11(10), 697-709. doi:10.1038/nrn2889

Tamietto, M., Geminiani, G., Genero, R., \& de Gelder, B. (2007). Seeing fearful body language overcomes attentional deficits in patients with neglect. Journal of Cognitive Neuroscience, 19(3), 445-54. doi:10.1162/jocn.2007.19.3.445

Tamietto, M., Pullens, P., de Gelder, B., Weiskrantz, L., \& Goebel, R. (2012). Subcortical connections to human amygdala and changes following destruction of the visual cortex. Current Biology, 22(15), 1449-55. doi:10.1016/j.cub.2012.06.006

Urgesi, C., Berlucchi, G., \& Aglioti, S. M. (2004). Magnetic Stimulation of Extrastriate Body Area Impairs Visual Processing of Nonfacial Body Parts Cosimo. Current Biology, 14, 2130-2134. doi:10.1016/j

Urgesi, C., Calvo-Merino, B., Haggard, P., \& Aglioti, S. M. (2007). Transcranial magnetic stimulation reveals two cortical pathways for visual body processing. The Journal of Neuroscience, 27(30), 80238030. doi:10.1523/JNEUROSCI.0789-07.2007 
Urgesi, C., Candidi, M., Ionta, S., \& Aglioti, S. M. (2007). Representation of body identity and body actions in extrastriate body area and ventral premotor cortex. Nature Neuroscience, 10(1), 30-31. doi:10.1038/nn1815

Van den Stock, J., Tamietto, M., Sorger, B., Pichon, S., Grézes, J., \& de Gelder, B. (2011). Corticosubcortical visual, somatosensory, and motor activations for perceiving dynamic whole-body emotional expressions with and without striate cortex (V1). Proceedings of the National Academy of Sciences of the United States of America, 108(39), 16188-93. doi:10.1073/pnas.1107214108

Van Loon, A. M., van den Wildenberg, W. P. M., van Stegeren, A. H., Hajcak, G., \& Ridderinkhof, K. R. (2010). Emotional stimuli modulate readiness for action: a transcranial magnetic stimulation study. Cognitive, Affective \& Behavioral Neuroscience, 10(2), 174-81. doi:10.3758/CABN.10.2.174

Zhan, M., Hortensius, R., \& Gelder, B. De. (2015). The body as a tool for anger awareness - Differential effects of angry facial and bodily expressions on suppression from awareness. Manuscript submitted for revision. 



\section{Chapter 3 \\ Dynamic interactions between emotion perception and motor preparation networks for reacting to social threat: a combined cTBS-fMRI study}

Based on:

Engelen, T., Zhan, M., Sack, A.T., \& de Gelder, B. (in revision). Dynamic interactions be- tween emotion perception and motor preparation networks for responding to social threat: a combined c'TBS-fMRI study. 



\begin{abstract}
Expressions of emotion are powerful triggers for situation-appropriate responses. Of particular interest regarding the preparation of such adaptive actions are parietal and premotor cortices, given their ability to interact with the amygdala (AMG), a central player in affective information processing. Our goal was to clarify the respective roles of inferior parietal lobule (IPL) and ventral premotor cortex (PMv) in the processing of emotional body expressions by assessing remote effects of continuous theta burst stimulation (cTBS) in the action network and AMG. Participants were presented with blocks of dynamic stimuli depicting either angry or neutral whole body actions in three separate fMRI sessions (two preceded by of either IPL or PMv stimulation, and a third baseline session). Interestingly, whereas at baseline the 1AMG did not differentiate between neutral and angry body postures, a significant difference between these conditions emerged after stimulation of either IPL or PMv, with much larger responses to angry than neutral stimuli. In addition, effects of cTBS stimulation and emotion expression were also observed in two other action relevant areas, supplementary motor area and superior parietal cortex. Our findings show the dynamic interaction between areas involved in action and emotion perception and adaptive behavior.
\end{abstract}

\title{
Introduction
}

Emotional expressions not only communicate affective information to the observer, but foremost they are powerful triggers for adapting one's behavior. A threatening body expression will likely prompt an appropriate reaction in the observer. Previous studies have shown that readying the brain for dealing with emotional signals involves visual processes, but also altered activity in motor structures (Borgomaneri, Vitale, \& Avenanti, 2015; Borgomaneri, Vitale, Gazzola, \& Avenanti, 2015; de Gelder, Snyder, Greve, Gerard, \& Hadjikhani, 2004). The inferior parietal lobule (IPL) is ideally located to play the role of a hub in which emotion perception is transitioned into such an action response. IPL receives input from the visual system (Caspers et al., 2011) and has connections to premotor cortex (PM) (Hoshi \& Tanji, 2007; Makris et al., 2005; Mars et al., 2011), where actions are prepared. Additionally, animal research shows connections between IPL and amygdala (AMG)(Clower, West, Lynch, \& Strick, 2001), which is a key region for threat processing and adaptive action preparation (Pessoa, 2010). Presumably, given this role, IPL differentiates between neutral and emotional actions and fast responses to the latter are crucial. Indeed, evidence for this was provided using MEG. Meeren et al. (2016) showed a differential response in right PPC to fearful body postures, compared to neutral, as early as $80 \mathrm{~ms}$ post stimulus onset. A causal involvement of IPL in emotion body processing was first demonstrated by applying online TMS during a delayed-match-to-sample task. Results showed an enhancement in performance in a fearful body condition (Engelen, de Graaf, Sack, $\&$ de Gelder, 2015), providing behavioural evidence for an increased sensitivity to emotional body stimuli under conditions of IPL stimulation.

PM is involved in action preparation and execution (Hoshi \& Tanji, 2007; Picard \& Strick, 2001), with specifically the ventral subpart (PMv) involved in space perception and understanding of actions (Rizzolatti, Fogassi, \& Gallese, 2002; Urgesi, Calvo-Merino, Haggard, \& Aglioti, 2007; Urgesi, Candidi, Ionta, \& Aglioti, 2007). Also in the case of PMv, several neuroimaging experiments have found emotion-specific activation (Calbi, Angelini, Gallese, \& Umiltà, 2017; Kret, Pichon, Grèzes, \& de Gelder, 2011; Pichon, de Gelder, \& Grezes, 2008), and one TMS study targeting PM found an increase in reaction times and false alarms specifically in response to faces expressing fear or anger (Balconi \& Bortolotti, 2013). 
In view of that emotional signals trigger activity in IPL and PM, a further question concerns the mechanism whereby these areas are influenced by affective information in the stimulus. Generally, the AMG is viewed as a major player in processing stimulus valence (Ledoux, 2000), and this is also found when observers view whole body expressions of threat (Hadjikhani \& de Gelder, 2003). More specifically, recent work has highlighted the importance of functional connectivity between AMG and (sensori-)motor regions (Grèzes, Valabrègue, Gholipour, \& Chevallier, 2014; Toschi, Duggento, \& Passamonti, 2017). This leads to the intriguing hypothesis that under conditions of perceived social threat, the amygdala is functionally engaging with areas related to action, specifically inferior parietal lobe and ventral premotor cortex, in order to effectively decode emotional content and at the same time allow for adaptive behaviour.

In order to directly test such emotion-dependent dynamic interaction between action related areas and AMG, we here employed an offline combined TMS-fMRI design to specifically manipulate neural activity within IPL and PMv using cTBS, and assessing network interaction effects within this motor-emotion circuitry during the passive viewing of either neutral or angry body emotions.

During three fMRI sessions, participants either received offline stimulation over IPL or PMv, or no stimulation (baseline fMRI). During the acquisition of fMRI data, immediately following stimulation, participants were presented with dynamic stimuli depicting either angry or neutral whole body actions. We expected to find an interaction effect between the site of stimulation and valence of the presented stimulus, demonstrating the emotion-specific interplay between amygdala and action related areas of the brain to i) differentiate between the valence of the stimuli, and to ii) valence-specific mediating role of parietal and motor areas on amygdala response patterns.

\section{Methods}

\section{Participants}

Seventeen healthy participants completed all three sessions of the experiment ( 5 males, mean age $(\mathrm{sd})=23(2.2)$ ). Fifteen were right handed and all had normal or corrected to normal vision. Participants signed a written consent form before participating and were rewarded for their participation in vouchers. Each participant was screened for fMRI and TMS safety and none had a history of neurological disorders. Given the demanding design of the experiment (immediately entering the scanner after receiving cTBS), only participants with prior TMS experience were recruited. Additionally, in order to have counterbalancing order of the sessions a pre-existing T1 image was necessary for localizing the stimulation sites, and for this purpose we recruited participants that had previously participated in other fMRI experiments. The study was approved by the local ethics committee and performed in accordance to the Declaration of Helsinki and approved by the local ethical committee.

\section{Stimuli}

The same stimulus data set was used as reported in Kret et al. (2011). Stimuli consisted of short 1 second video clips showing male actors performing whole body movements of either an angry or neutral nature in front of a green screen. For the neutral movement the actors brought their hand in front of their face while coughing, while angry videos depicted the actor moving 
up his fists in front of his trunk. Eleven different clips were used performed by six different actors. The clips were presented without sound and stimulus size was 720 x 576 pixels.

\section{TMS stimulation and site localization}

Before the start of two of the functional MRI session, cTBS ( 3 pulses at $50 \mathrm{~Hz}$, every $200 \mathrm{~ms}$ for a total of 600 pulses (Huang, Edwards, Rounis, Bhatia, \& Rothwell, 2005)) was applied at $80 \%$ of active motor threshold (mean intensity(sd) $=25(3.5) \mathrm{MSO}$ ) using a MC-B70 figure-ofeight coil and Magpro X100 stimulator (Medtronic Functional Diagnostics A/S, Skovlunde, Denmark). Either right ventral premotor cortex (rPMv) or right inferior parietal lobule (rIPL) were targeted based on individual macroanatomical landmarks.

The rIPL was located by identifying a point that lies directly posterior to the intraparietal sulcus (IPS) at the caudal end of the posterior branch of superior temporal sulcus (the same approach as used in Engelen et al. (2015). For rPMv, we selected a point directly below the intersection of inferior frontal sulcus and precentral gyrus (a similar approach as used by Cattaneo et al. (2010) and Tremblay, Sato, \& Small (2012)) . See table 1 for the average Talairach coordinates per stimulation site and figure 1 for a representation of individual stimulation sites represented in Talairach space. For both stimulation sites the coil was positioned with the handle pointing backward and outward at a $45^{\circ}$ angle from the mid-sagittal axis.

Table 1. Average Talairach coordinates per stimulation site \pm standard deviation

\begin{tabular}{llll}
\hline Stimulation site & $\mathbf{x}$ & $\mathbf{y}$ & $\mathbf{z}$ \\
\hline \hline Inferior parietal lobule & $41 \pm 7$ & $-62 \pm 9$ & $43 \pm 5$ \\
Ventral premotor cortex & $50 \pm 6$ & $11 \pm 4$ & $29 \pm 5$ \\
\hline
\end{tabular}




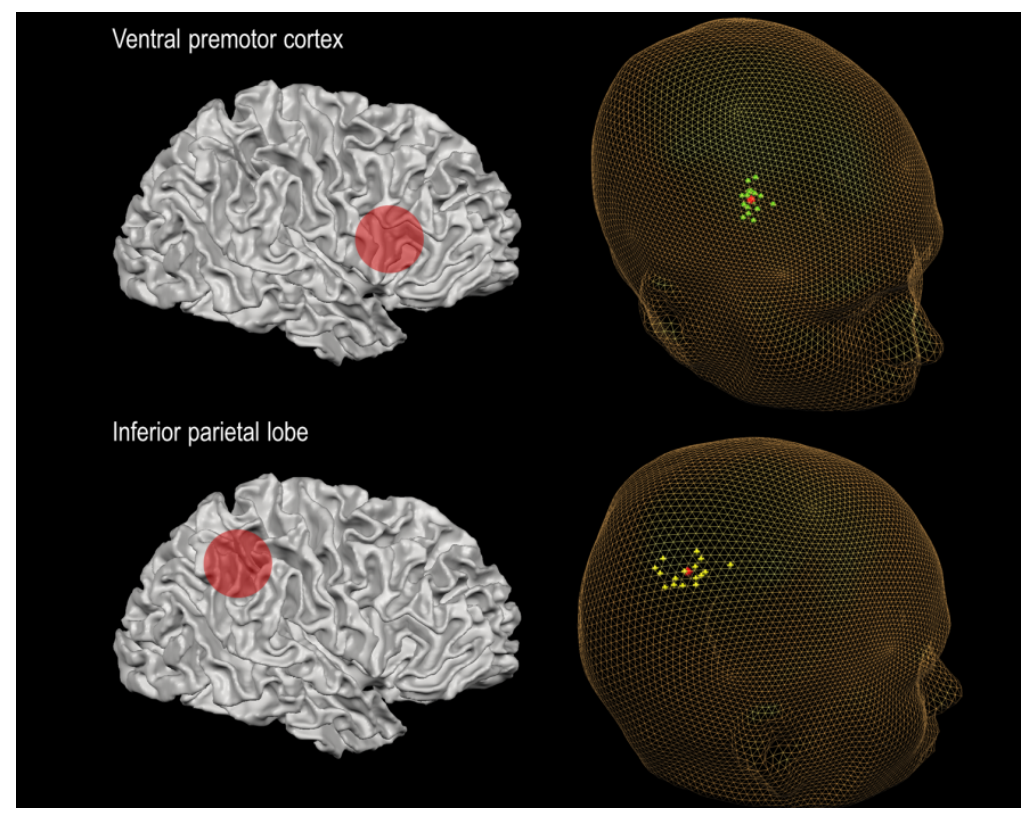

Figure 1. The two stimulation sites for cTBS consisted of the right ventral premotor cortex and right inferior parietal lobule. Both target points were localized using individual brain anatomy (see TMS stimulation and site localization for details) and cTBS stimulation was guided by neuronavigation. Individual target points are displayed in Talairach space for rPMv (green) and rIPL (yellow). The red spheres indicate the average stimulation coordinates.

\section{Procedure}

The experiment consisted of three sessions, of which the order was randomized, performed on separate days with at least one week in between each session. During two of the sessions cTBS was applied over either rIPL or rPMv. During these TMS sessions, motor threshold was established by moving the coil over primary motor cortex until an optimal position was found for eliciting muscle twitches in the hand muscles. After this, the stimulation intensity was decreased until a threshold was found at which 5 out of 10 pulses still evoked a motor response while there was some tension on the hand muscles. Next, the correct coil location on the scalp was determined for each stimulation site by using the BrainVoyager TMS Neuronavigator software (Brain Innovation, Maastricht, The Netherlands). Once the coil was in the correct position, the cTBS protocol was applied, after which participants were moved to the scanner environment as fast as possible (the first functional run was started $<10$ minutes after stimulation). The participant was placed in the scanner and two functional runs were acquired in which the stimuli portraying dynamic neutral or angry actions were presented. Each functional run lasted 8 minutes (16 minutes of task in total), so the total scanning time fell within the window of assumed effectivity for cTBS (cTBS effects are reported to last up to 60 minutes if the protocol for application of 40 seconds (Huang et al., 2005)). The session in which no stimulation was 
applied followed the same scanning procedure, with the addition of two functional localizers at the end of the scan (see functional localizers for details).

\section{Scanning parameters}

Functional images were acquired using a 3T MAGNETOM Prisma fit scanner (Siemens, Erlangen, Germany) with a 64-channel head-neck coil. A gradient-echo EPI sequence was used (repetition time $(T R)=2 \mathrm{sec}$, echo time $(T E)=31 \mathrm{msec}$, voxel size $=2 \times 2 \times 2 \mathrm{~mm}^{3}, 64$ slices, no gaps, multi-band acceleration factor $=2$, flip angle $(\mathrm{FA})=77^{\circ}$ ), providing whole-brain coverage. A high-resolution T1-weighted MPRAGE anatomical scan was acquired for each participant during the baseline session (voxel size $=1 \times 1 \times 1 \mathrm{~mm}, 192$ slices).

The main experiment started with a fixation period of 6 TRs, followed by blocks of stimuli of 6 TRs separated by fixation periods of 6 TRs, and ending with a fixation period of 12 TRs. During each block a total of 12 dynamic whole body stimuli were shown, either depicting angry or neutral movements. The main task contained two functional runs; during each run 16 blocks of stimuli were presented. Per session a total of 432 volumes were acquired for the main task.

\section{Functional localizers}

To identify individual ROIs of extrastriate body area (EBA), the same functional localizer was used as described in Engelen et al. (2015). Static images of five different categories of images (bodies, faces, houses, tools and words) were shown pseudo-randomized in a block design, with 7 blocks per condition. Each block lasted 6 TRs interspersed with fixation periods of 6 TRs. The same scanning parameters as in the main task were used. A total of 432 volumes were acquired for this localizer. To identify bilateral EBA, a contrast was made between the responses to bodies and houses, and the cluster was selected that showed the greatest relative activation for the bodies' condition (in case more than one cluster was observed in the same hemisphere, the clusters were merged into one).

In order to determine a functional ROI in right posterior superior temporal sulcus (rpSTS), another localizer was used in which short dynamic clips of male faces and bodies were presented in a block design. Each block consisted of eight 1 second dynamic stimuli, lasting a total of 4 TRs. These blocks were interspersed with fixation periods of 4TRs. In total 10 blocks of each stimulus condition were presented and a total of 168 volumes were acquired for this localizer.

\section{Data pre-processing}

Functional and anatomical brain imaging data were pre-processed and analysed offline using BrainVoyager QX (Brain Innovation BV, Maastricht, The Netherlands). Functional data was slice scan time corrected and 3D motion corrected using sinc interpolation. To correct for low frequency drifts in the data, high-pass temporal filtering was applied. Spatial smoothing with a Gaussian kernel of $6 \mathrm{~mm}$ FWHM was applied to the acquired images in the main task, and smoothing of $4 \mathrm{~mm}$ was applied to the functional localizers. All functional runs of the three separate sessions were aligned to the anatomical scan acquired during the baseline session. To enable analyses on the group level, individual data was spatially transformed into Talairach space. A brain mask was created by averaging the first volume of each functional run of all 
participants and removing the scalp. This mask was applied during the cluster threshold correction.

\section{GLM analysis}

First, for each individual participant a GLM map was created for each of the sessions using the predictors 'Angry bodies' and 'Neutral bodies'. Z-transformed 3D motions parameters were added as confound predictors in the model. The maps of all participants and sessions were combined as inputs for a random effects (RFX) ANOVA group analysis. This RFX ANOVA included the factors emotion (neutral and angry bodies) and session (reflecting the different stimulation conditions (cTBS over rIPL, cTBS over rPMv or baseline)). The resulting maps revealed by the ANOVA were corrected for multiple comparisons by cluster threshold estimation (alpha $=.05$, initial threshold set at $\mathrm{p}=.001$, Monte Carlo simulation $\mathrm{n}=5000$ ).

\section{ROI analysis}

For each participant bilateral amygdalae were segmented based on the anatomical data, in order to isolate any effects of emotion and cTBS in the amygdalae. To assess the local effects of cTBS stimulation, for each participant a ROI for rIPL and rPMv was created. This was done by first creating a grey matter segmentation of the right hemisphere, and then placing a sphere ROI of $10 \mathrm{~mm}^{3}$, centred at the individual stimulation coordinates. For each of the segmented AMG clusters, the rIPL and rPMv ROIs, as well as the functionally determined ROIs for bilateral EBA and rpSTS, beta values (percent signal changes) were extracted from the functional runs for each emotion (neutral, angry) and stimulation condition (cTBS over rIPL, cTBS over $\mathrm{rPMv}$, or baseline). These beta values were then analysed in a $2 \times 3$ repeated measures ANOVA.

\section{Results}

Whole-brain random effects $A N O V A$

\section{Main effect of emotion}

Clusters showing a main effect of emotion were observed in bilateral fusiform gyri, anterior inferior frontal gyrus, bilateral amygdala, right pulvinar and superior parieto-occipital cortex (SPOC). For an overview of all clusters see table 2. For all clusters activation for angry bodies was stronger than neutral bodies, except for an occipital cluster in the right hemisphere (cluster 5 in table 2), for which there was a stronger activation for neutral than for angry bodies. 
Table 2. Clusters found for the main effect of emotion (alpha $=.05$, cluster size corrected). Cluster size is reported in number of voxels (voxel size is $1 \mathrm{~mm}^{3}$ ). Reported values (coordinates, F-value and $p$-value) reflect cluster peak.

\begin{tabular}{|c|c|c|c|c|c|c|c|}
\hline Anatomical description & Hemisphere & Cluster size & $\mathbf{x}$ & $\mathrm{y}$ & $\bar{z}$ & $F$-value & $P$-value \\
\hline $\begin{array}{l}\text { widespread occipital/fusi- } \\
\text { form gyrus }\end{array}$ & $\mathrm{R}$ & 16826 & 43 & -67 & 0 & 85.09 & 0.000000 \\
\hline $\begin{array}{l}\text { anterior inferior temporal } \\
\text { gyrus }\end{array}$ & $\mathrm{R}$ & 363 & 36 & -5 & -36 & 45.78 & 0.000005 \\
\hline amygdala & $\mathrm{R}$ & 347 & 25 & -1 & -12 & 56.63 & 0.000001 \\
\hline pulvinar & $\mathrm{R}$ & 354 & 17 & -27 & 2 & 45.70 & 0.000005 \\
\hline $\begin{array}{l}\text { superior parieto-occipital } \\
\text { cortex }\end{array}$ & $\mathrm{R} / \mathrm{L}$ & 2864 & 1 & -59 & 0 & 54.17 & 0.000002 \\
\hline $\begin{array}{l}\text { widespread occipital/fusi- } \\
\text { form gyrus }\end{array}$ & $\mathrm{L}$ & 22222 & -15 & -89 & -2 & 75.61 & 0.000000 \\
\hline amygdala & $\mathrm{L}$ & 697 & -35 & -1 & -12 & 45.41 & 0.000005 \\
\hline
\end{tabular}

\section{Main effect of session}

Clusters showing a main effect of session (baseline, cTBS over rIPL or cTBS over rPMv) were observed in right ventromedial prefrontal cortex (vmPFC), left posterior cingulate cortex (PCC), left precuneus, left superior parietal lobe (SPL) and left PMv. The cluster in PMv additionally showed an emotion $*$ session interaction, details of which are reported in the section discussing the interaction findings.

Pairwise comparisons showed that the clusters in vmPFC and PCC showed lower activity after cTBS over both $\mathrm{rPMv}(p=.007$ and $p=.033$ respectively, Sidak correction) and rIPL $(p=.001$ and $p<.001)$ compared to baseline, whereas the cluster in precuneus showed lower activity after cTBS over rIPL compared to both baseline $(p=.003)$ and cTBS over rPMV $(p=.003)$. The cluster in SPL significantly decreased in activation after cTBS over rPMv compared to baseline $(p=.002)$ and cTBS over rIPL $(p=.002)$. Lastly, the cluster in IPMv showed an increase in activation after cTBS over rIPL compared to both other sessions $(p=.002$ and $p=$ .008). For a detailed overview of all clusters found for the main effect of session see table 3 and figure 2 . 


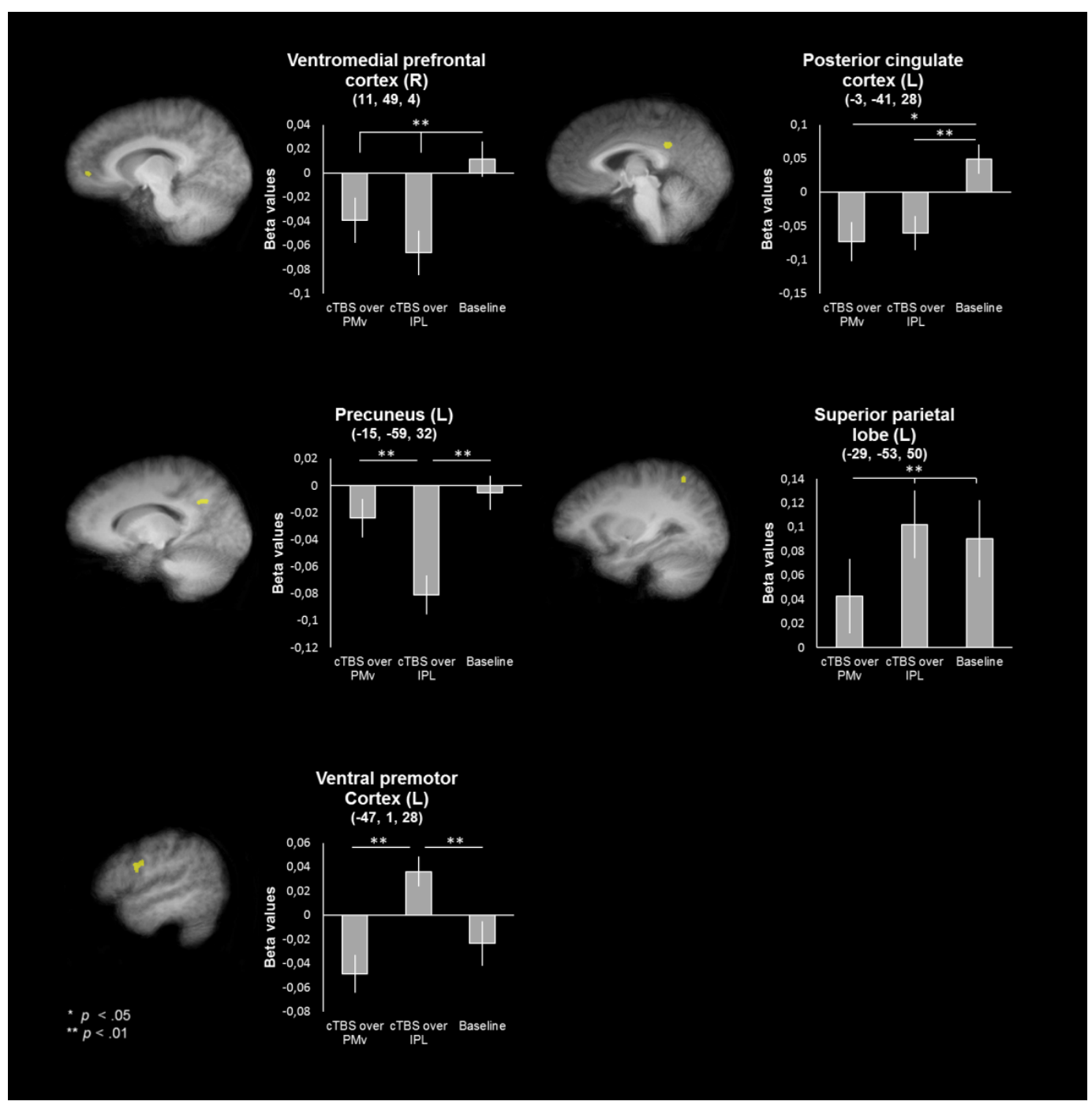

Figure 2. Clusters showing a significant main effect of session displayed on an averaged anatomy of all participants in Talairach space, $\mathrm{p}=.05$, cluster size corrected. For details about cluster size and statistical significance see table 3 . 
Table 3. Clusters found for the main effect of session (alpha $=.05$, cluster size corrected). Cluster size is reported in number of voxels (voxel size is $1 \mathrm{~mm}^{3}$ ). Reported values (coordinates, F-value and $p$-value) reflect cluster peak.

\begin{tabular}{llllllll}
\hline Anatomical description & Hemisphere & Cluster size & $\mathbf{x}$ & $\mathbf{y}$ & $\mathbf{z}$ & $F$-value & $P$-value \\
\hline \hline ventromedial prefrontal & & 112 & 11 & 49 & 4 & 15.37 & 0.000021 \\
cortex & $\mathrm{R}$ & 197 & -3 & -41 & 28 & 14.87 & 0.000027 \\
posterior cingulate cortex & $\mathrm{L}$ & 108 & -15 & -59 & 32 & 11.97 & 0.000131 \\
precuneus & $\mathrm{L}$ & 107 & -29 & -53 & 50 & 15.47 & 0.000020 \\
superior parietal lobe & $\mathrm{L}$ & 255 & -47 & 1 & 28 & 11.73 & 0.000151 \\
ventral premotor cortex & $\mathrm{L}$ & & & & & & \\
\hline
\end{tabular}

\section{Emotion by session interaction effect}

Clusters reflecting an emotion x session interaction effect were found in right lingual gyrus, bilateral SPL, left SPOC, cerebellum, precuneus and left PMv. Post hoc tests were completed for each cluster to disentangle the significant differences underlying the interaction, for a visual overview of these significant differences see figure 3 .

The cluster in the lingual gyrus showed a main effect for emotion in all three sessions $(\mathrm{F}(1,16)$ $=44.153, p<.001, \eta_{\mathrm{p}}^{2}=0.734$ in the PMv session, $\mathrm{F}(1,16)=9.460, p=.007, \eta_{\mathrm{p}}^{2}=0.372$ in the IPL session and $\mathrm{F}(1,16)=7.037, p=.017, \eta_{\mathrm{p}}^{2}=0.305$, in the baseline session). To further follow up on the interaction and see if the difference between angry and neutral bodies became relatively bigger between sessions, we calculated the difference between the angry condition of two sessions and subtracted from that the difference between the neutral conditions of the same sessions. T-tests (two-tailed) showed that the difference between angry and neutral is relatively bigger when comparing PMv to baseline $(t(16)=2.896, p=.011)$ and to IPL $(t(16)$ $=2.794, p=.013)$, but not when comparing IPL to baseline $(t(16)=0.642, p=.530)$.

The cluster in right SPL showed a significant main effect of emotion in the PMv session (F $\left.(1,16)=5.567, p=.031, \eta_{\mathrm{p}}^{2}=.258\right)$, the IPL session $\left(\mathrm{F}(1,16)=14.331, p=.002, \eta_{\mathrm{p}}^{2}=0.472\right)$ and the baseline session $\left(\mathrm{F}(1,16)=9.775, p=.007, \eta_{\mathrm{p}}^{2}=0.379\right)$. Within the angry condition there was a main effect of session $\left(\mathrm{F}(2,32)=4.104, p=.026, \eta_{\mathrm{p}}^{2}=0.204\right)$, caused by differences between he PMv and baseline session $(p=.015)$. In the neutral condition there was also a main effect of session $\left(\mathrm{F}(2,32)=5.120, p=.012, \eta_{\mathrm{p}}^{2}=0.242\right)$, driven by a significant difference between the PMv and IPL sessions $(p=.015)$.

The cluster in SMA showed a main effect of emotion in the PMv session $(F(1,16)=15.652, p$ $\left.=.001, \eta_{\mathrm{p}}^{2}=0.494\right)$ and the baseline session $\left(\mathrm{F}(1,16)=10.038, p=.006, \eta_{\mathrm{p}}^{2}=0.385\right)$, but not in the IPL session $\left(\mathrm{F}(1,16)=0.685, p=.420, \eta_{\mathrm{p}}^{2}=0.041\right)$. Within the angry condition there was a main effect of session $\left(\mathrm{F}(2,32)=3.912, p=.030, \eta_{\mathrm{p}}^{2}=0.196\right)$, caused by differences between the PMv and baseline session $(p=.040)$. In the neutral condition there was no main effect of session $\left(\mathrm{F}(2,32)=3.041, p=.062, \eta^{2}=0.160\right)$.

The cluster in left SPL showed a main effect of emotion within the IPL $(\mathrm{F}(1,16)=11.441, p=$ $\left..004, \eta_{\mathrm{p}}^{2}=0.417\right)$ and baseline sessions $\left(\mathrm{F}(1,16), 10.824, p=.005, \eta_{\mathrm{p}}^{2}=0.404\right)$, but not in the 
PMv session $\left(\mathrm{F}(1,16)=3.825, p=.068, \eta^{2} \mathrm{p}=0.193\right)$. Within the angry condition there was no main effect of session $\left(\mathrm{F}(2,32)=1.794, p=.183, \eta_{\mathrm{p}}^{2}=0.101\right)$. Within the neutral body condition however, there was a main effect of session $\left(\mathrm{F}(2,32)=10.256, p<.001, \eta_{\mathrm{p}}{ }_{\mathrm{p}}=0.391\right)$, driven by significant differences between the PMv and IPL sessions $(p<.001)$ and PMv and baseline sessions $(p=.015)$.

The cluster in SPOC showed a main effect of emotion in the PMv $(\mathrm{F}(1,16)=17.390, p=.001$, $\left.\eta_{\mathrm{p}}^{2}=0.521\right)$ and baseline sessions $\left(\mathrm{F}(1,16)=5.780, p=.029, \eta_{\mathrm{p}}^{2}=0.265\right)$, but not in the IPL session $\left(\mathrm{F}(1,16)=3.529, p=.079, \eta_{\mathrm{p}}^{2}=0.181\right)$. Within the angry condition there was a main effect of session $\left(\mathrm{F}(2,32)=3.664, p=.037, \eta_{\mathrm{p}}^{2}=0.186\right)$, driven by a significant difference between the PMv and IPL sessions $(p=.019)$. Within the neutral condition there was a main effect of session $\left(F(2,32)=4.336, p=.022, \eta^{2}{ }_{\mathrm{p}}=0.213\right)$, driven by differences between the $\mathrm{PMv}$ and baseline session $(p=.007)$.

The cluster in cerebellum only showed a main effect of emotion in the PMv session $(\mathrm{F}(1,16)$ $\left.=8.885, p=.009, \eta_{\mathrm{p}}^{2}=0.357\right)$, but not in the IPL $\left(\mathrm{F}(1,16)=0.433, p=.520, \eta_{\mathrm{p}}^{2}=0.026\right)$ or baseline $\left(\mathrm{F}(1,16)=0.095, p=.761, \eta_{\mathrm{p}}^{2}=0.006\right)$ sessions. There was no main effect of session in either the angry $\left(\mathrm{F}(2,32)=1.110, p=.342, \eta_{\mathrm{p}}^{2}=0.065\right)$ or neutral $(\mathrm{F}(2,32)=0.648, p=.530$, $\left.\eta_{\mathrm{p}}^{2}=0.039\right)$ condition.

The cluster in precuneus showed a main effect of emotion in both the $\mathrm{PMv}(\mathrm{F}(1,16)=9.309$, $\left.p=.008, \eta_{\mathrm{p}}^{2}=0.368\right)$ and the IPL $\left(\mathrm{F}(1,16)=10.222, p=.006, \eta_{\mathrm{p}}^{2}=0.390\right)$ sessions, but not at baseline $\left(\mathrm{F}(1,16)=1.673, p=.214, \eta_{\mathrm{p}}^{2}=0.095\right)$. Within the angry condition there was no main effect of session $\left(\mathrm{F}(2,32)=0.456, p=.638, \eta^{2} \mathrm{p}=0.028\right)$. Within the neutral condition there was a main effect of session however $\left.(\mathrm{F}, 2,32)=7.416, p=.002, \eta_{\mathrm{p}}^{2}=0.317\right)$, driven by differences between the PMv and IPL sessions $(p=.016)$, as well as between the PMv and baseline sessions $(p=.016)$.

The cluster in $1 \mathrm{PMv}$ showed a main effect of emotion in each of the sessions $(\mathrm{PMv}(\mathrm{F}(1,16)=$ 7.238, $\left.p=.016, \eta_{\mathrm{p}}^{2}=0.311\right)$, IPL $\left(\mathrm{F}(1,16)=7.038, p=.017, \eta_{\mathrm{p}}{ }_{\mathrm{p}}=0.305\right)$, and at baseline $\left.\left(\mathrm{F}(1,16)=9.313, p=.008, \eta_{\mathrm{p}}^{2}=0.368\right)\right)$. In addition, there is a main effect of session within the angry condition $\left(\mathrm{F}(2,32)=8.298, p=.001, \eta_{\mathrm{p}}^{2}=0.342\right)$, driven by the difference between the IPL and baseline sessions $(p=.006)$. Within the neutral condition there is also a main effect of session $\left(\mathrm{F}(2,32)=6.824, p=.003, \eta^{2} \mathrm{p}=0.299\right)$, driven by differences between both the PMv and IPL sessions $(p=.046)$, as well as between the PMv and baseline sessions $(p=.003)$. 
Table 4. Clusters found for the emotion $\mathrm{x}$ session interaction (alpha $=.05$, cluster size corrected). Cluster size is reported in number of voxels (voxel size is $1 \mathrm{~mm}^{3}$ ). Reported values (coordinates, F-value and $p$-value) reflect cluster peak.

\begin{tabular}{llllllll}
\hline Anatomical description & Hemisphere & Cluster size & $\mathbf{x}$ & $\mathbf{y}$ & $\mathbf{z}$ & $F$-value & $P$-value \\
\hline \hline lingual gyrus & $\mathrm{R}$ & 287 & 17 & -77 & -8 & 13.76 & 0.000049 \\
superior parietal lobe & $\mathrm{R}$ & 1077 & 15 & -73 & 44 & 17.98 & 0.000006 \\
supplementary motor area & $\mathrm{R} / \mathrm{L}$ & 505 & -1 & 3 & 54 & 12.77 & 0.000084 \\
superior parietal lobe & $\mathrm{L}$ & 802 & -13 & -69 & 48 & 15.47 & 0.000020 \\
superior parieto-occipital cortex & $\mathrm{L}$ & 340 & -17 & -65 & 26 & 14.99 & 0.000025 \\
cerebellum & $\mathrm{L}$ & 164 & -19 & -71 & -22 & 12.12 & 0.000120 \\
precuneus & $\mathrm{L}$ & 354 & -27 & -59 & 46 & 14.52 & 0.000032 \\
ventral premotor cortex & $\mathrm{L}$ & 113 & -49 & 9 & 26 & 13.20 & 0.000066 \\
\hline
\end{tabular}


Dynamic interactions between emotion perception and motor preparation networks

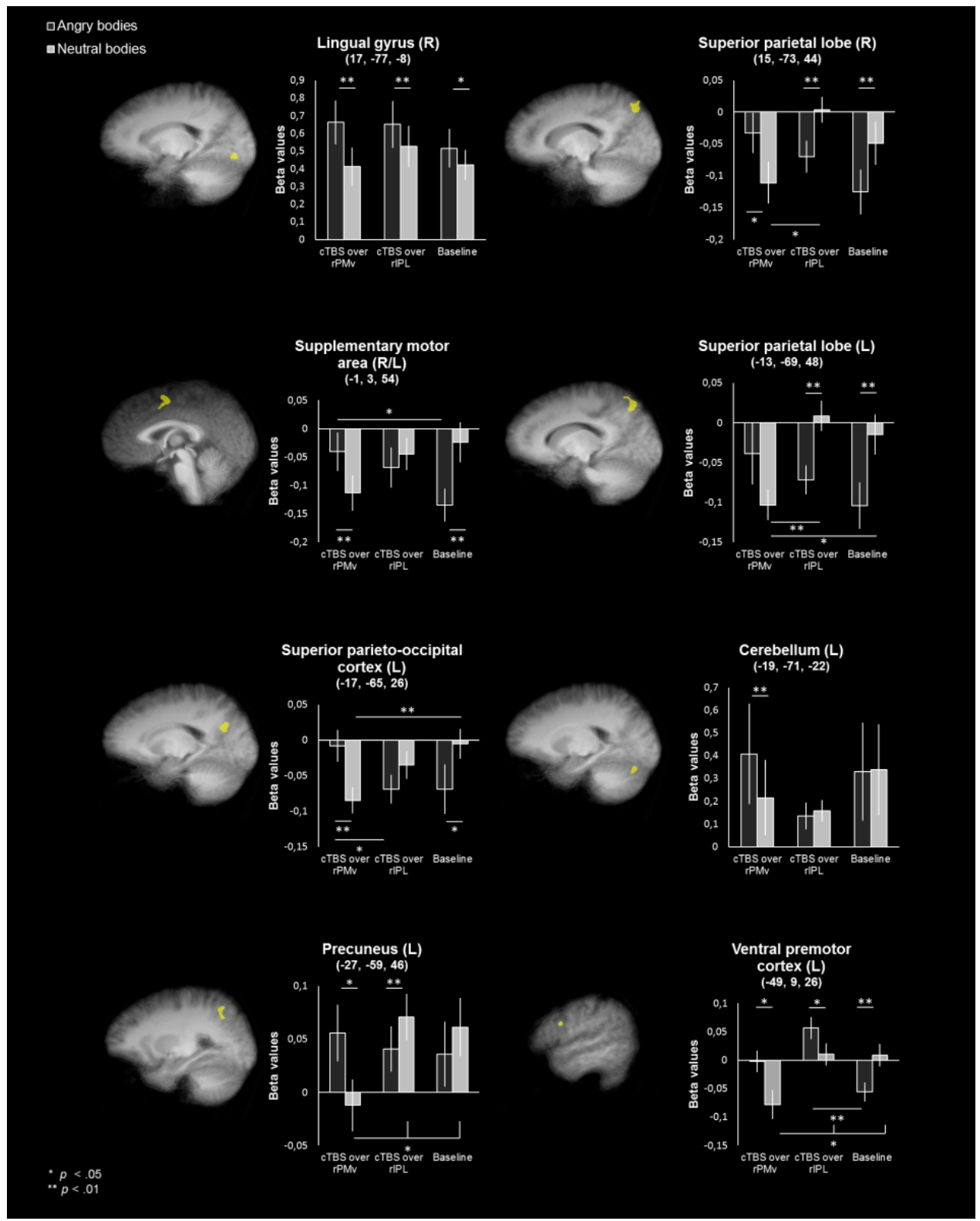

Figure 3. Clusters showing a significant emotion $\mathrm{x}$ session interaction displayed on an averaged anatomy of all participants in Talairach space, $\mathrm{p}=.05$, cluster size corrected. For details about cluster size and statistical significance see table 4 . 


\section{ROI analyses}

\section{Local effects of cTBS and emotion in rPMv and rIPL}

A $2 \times 3$ repeated measures ANOVA on the beta values in rPMv showed no main effect of emotion $\left(F(1,16)=0.603, p=.449, \eta_{\mathrm{p}}^{2}=.036\right)$, nor a main effect of session $(F(2,32)=0.902$, $\left.p=.416, \eta_{\mathrm{p}}^{2}=.053\right)$. There was however a significant emotion $\mathrm{x}$ session effect $(F(2,32)=$ $\left.3.896, p=.031, \eta_{\mathrm{p}}^{2}=.196\right)$. Post-hoc tests to disentangle the interaction effect showed no main effects of emotion after either PMv stimulation $\left(F(1,16)=2.771, p=.115, \eta_{\mathrm{p}}^{2}=.148\right)$, nor after IPL stimulation $\left(F(1,16)=2.322, p=.147, \eta_{\mathrm{p}}^{2}=.127\right)$, or during baseline $(F(1,16)=$ $\left.2.783, p=.115, \eta_{\mathrm{p}}^{2}=.148\right)$. Neither was there a main effect of session either in the angry $\left(F(1,16)=2.376, p=.109, \eta_{\mathrm{p}}^{2}=.129\right)$, or neutral condition $\left(F(1,16)=1.620, p=.214, \eta_{\mathrm{p}}^{2}=\right.$ $.092)$.

In the ROI for rIPL, there was no main effect of emotion $\left(F(1,16)=1.498, p=.239, \eta_{\mathrm{p}}^{2}=\right.$ $.086)$, no main effect of session $\left(F(2,32)=0.355, p=.704, \eta_{\mathrm{p}}^{2}=.022\right)$, and no emotion $\mathrm{x}$ session interaction $\left(F(2,32)=1.750, p=.190, \eta_{\mathrm{p}}^{2}=.099\right)$.

\section{Effect of $c T B S$ and emotion in the amygdalae}

A $2 \times 3$ repeated measures ANOVA on the beta values in 1 AMG showed a main effect of emotion $\left(F(1,16)=19.561, p<.001, \eta_{\mathrm{p}}^{2}=.550\right)$, as well as an emotion $\mathrm{x}$ session interaction effect $\left(F(2,32)=3.922, p=.030, \eta_{\mathrm{p}}^{2}=.197\right)$. There was no main effect of session $(F(2,32)=$ $\left.0.497, p=.613, \eta_{\mathrm{p}}^{2}=.030\right)$. Post-hoc tests to disentangle the interaction revealed that while both during the PMv and the IPL sessions there was a significant difference between angry and neutral bodies $\left(F(1,16)=13.533, p=.002, \eta_{\mathrm{p}}^{2}=.458\right.$, and $F(1,16)=19.085, p=>.001$, $\eta_{\mathrm{p}}^{2}=.544$, respectively), with stronger activation for angry than neutral bodies, this effect was absent during the baseline session $\left(\left(F(1,16)=.066, p=0.801, \eta_{\mathrm{p}}^{2}=.004\right)\right.$. In addition, pairwise comparison between sessions in the angry body condition showed that activation for angry bodies during the IPL session was significantly stronger than during the baseline session $(p=$ .033).

The analysis on the beta values in rAMG showed a main effect of emotion $(F(1,16)=6.67, p$ $=.020, \eta_{\mathrm{p}}^{2}=0.294$ ), with on average higher values for angry than neutral bodies (means are .064 and .040). There was no main effect of session $\left(F(2,32)=0.440, p=.648, \eta^{2}{ }_{\mathrm{p}}=.027\right)$, nor an emotion $\mathrm{x}$ session interaction $\left(F(2,32)=0.604, p=.553, \eta_{\mathrm{p}}^{2}=.036\right)$ (see figure 5 for the results in AMG).

Effect of cTBS and emotion in bilateral Extrastriate Body Area and right posterior Superior Temporal Sulcus A $2 \times 3$ repeated measures ANOVA on the beta values in $\mathrm{rEBA}$ revealed a main effect of emotion $\left(F(1,16)=51.701, p<.001, \eta_{\mathrm{p}}^{2}=.764\right)$, but no main effect of session $(F(2,32)=0.295$, $\left.p=.746, \eta_{\mathrm{p}}^{2}=.018\right)$, or emotion x session interaction $\left(F(2,32)=2.695, p=.083, \eta_{\mathrm{p}}^{2}=.144\right)$. Results in lEBA again showed a main effect of emotion $\left(F(1,16)=58.677, p<.001, \eta_{\mathrm{p}}^{2}=.786\right)$, but not a main effect of session $\left(F(2,32)=0.382, p=.685, \eta_{\mathrm{p}}^{2}=.023\right)$ nor an emotion $\mathrm{x}$ session interaction $\left(F(2,32)=2.834, p=.074, \eta_{\mathrm{p}}^{2}=.150\right)$.

Analysis on the beta values in rpSTS revealed a main effect of emotion $(F(1,16)=24.050, p<$ $\left..001, \eta_{\mathrm{p}}^{2}=.600\right)$, but no main effect of session $\left(F(2,32)=0.141, p=.869, \eta_{\mathrm{p}}^{2}=.009\right)$ or emotion 
$\mathrm{x}$ session interaction $\left(F(2,32)=1.458, p=.248, \eta_{\mathrm{p}}^{2}=.084\right)$. See figure 4 for all results of the ROI analysis.

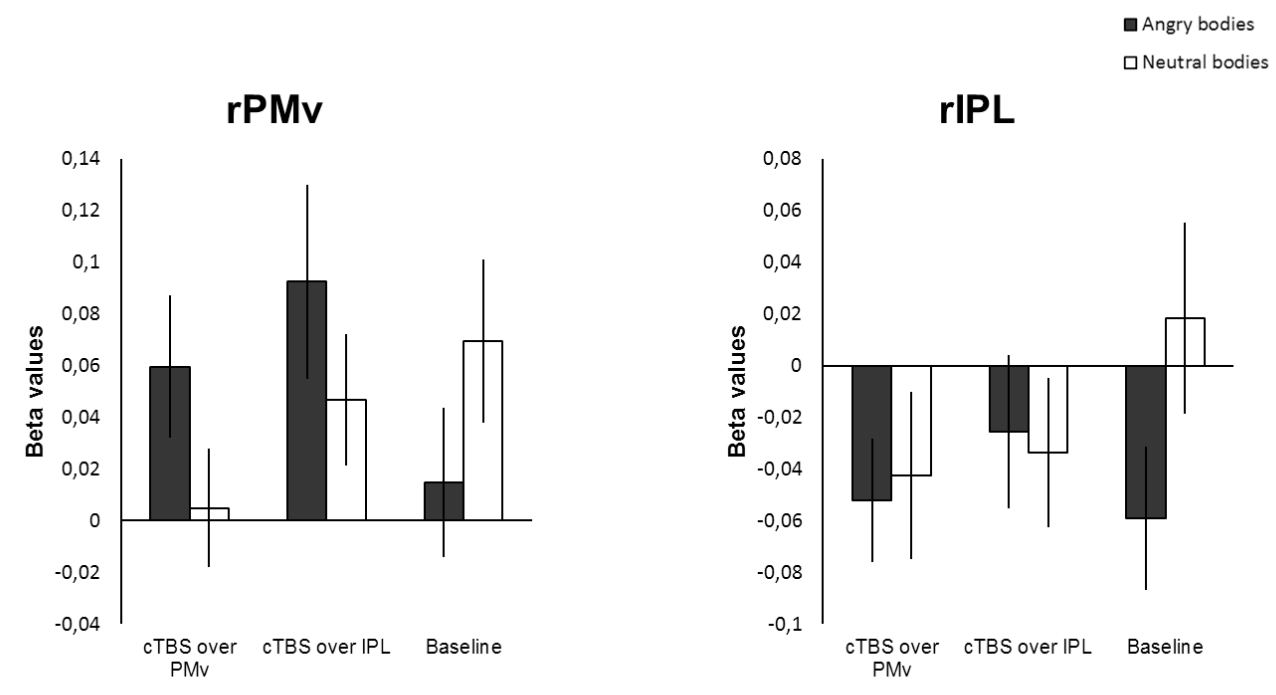

Figure 4. Results from ROI analysis based on individual stimulation sites (rPMv and rIPL). Beta values in rPMv showed a significant emotion $\mathrm{x}$ session interaction effect, whereas in rIPL no significant effects were found. 


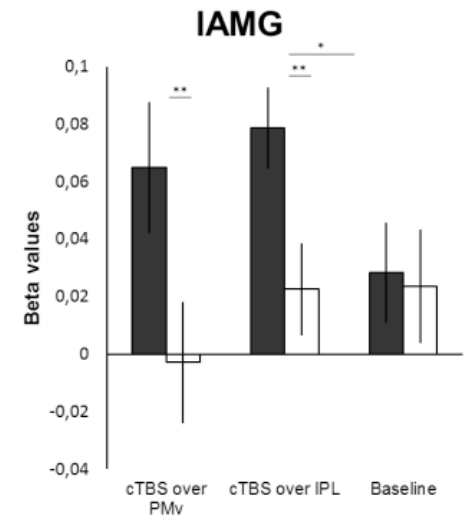

IEBA

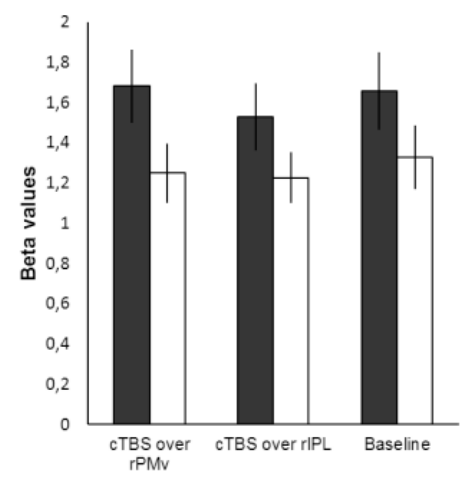

rpSTS

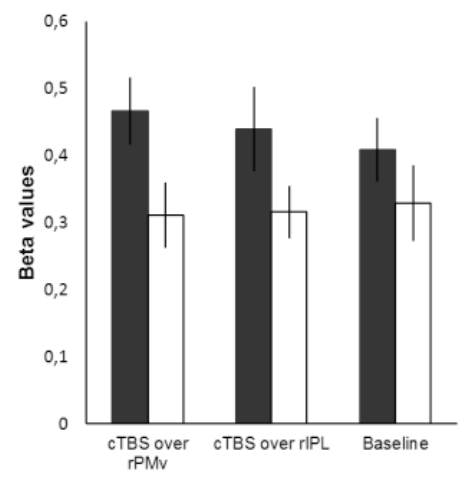

rAMG

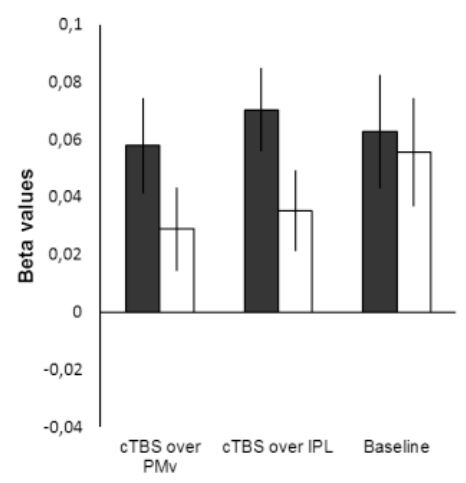

rEBA

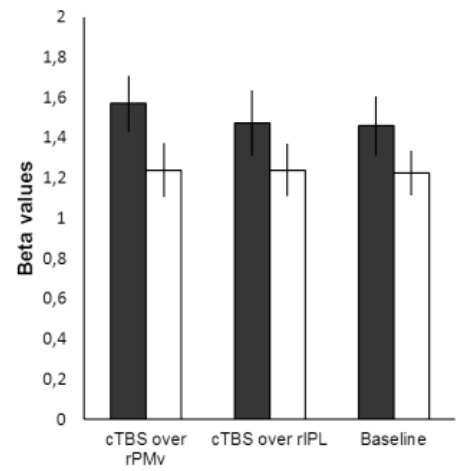

- Angry bodies

$\square$ Neutral bodies

${ }^{*} p<.05$

${ }^{* *} p<.01$

Figure 5. Results from ROI analysis based on individual anatomy (amygdala) or functional activity (EBA and pSTS). Beta values in rAMG, bilateral EBA and rpSTS reflected a main effect of emotion, whereas in 1 AMG also an interaction effect was present. In $1 \mathrm{AMG}$ there was no effect of emotion at baseline, but this effect was evident for both cTBS sessions. 


\section{Discussion}

We combined offline TMS and PMRI in order to test the hypothesis that IPL and PMv are involved in processing of affective body expressions, and measure possible interactions of these areas with AMG during processing of such information. ROI analysis in left AMG shows an interaction between the emotion portrayed in the stimuli and the stimulation site. Similar interactions were observed in clusters in SPL, PMv, SMA, cerebellum and occipital cortex. Concerning $1 \mathrm{AMG}$, whereas there was no significant difference between angry and neutral bodies at baseline, this difference was significant after cTBS over both rPMv and rIPL. Functionally determined ROIs in EBA and pSTS only showed main effects of emotion, and did not interact with the stimulated regions, as their pattern of activation was unaffected by cTBS over either of the stimulation sites.

\section{Processing of affective body expressions in the amygdalae}

Many classic studies have implicated the AMG as a region involved in the processing of threatening information (Ledoux, 2000). Much of the research on AMG function has focused on the processing of faces and fear, but more recently a broader picture of AMG function has emerged (Davis \& Whalen, 2001). It is now clear that the AMG plays a crucial role in the processing of emotional body stimuli (Hadjikhani \& de Gelder, 2003, Hortensius et al., 2017), as previous work has shown that contrasting neutral and fearful body expression results in significant activation in AMG (Hadjikhani \& de Gelder, 2003). However, results are conflicting, with other experiments finding a general effect of bodies (rather than valence) in AMG (Grèzes, Pichon, \& de Gelder, 2007; Pichon, de Gelder, \& Grezes, 2008). Recent literature suggests findings in AMG might depend on various task manipulations, such as task load and attention (de Gelder, Hortensius, \& Tamietto, 2012). In the current experiment, we compared passive viewing of dynamic neutral to angry bodies, and found no significant difference between these two conditions at baseline. Interestingly, our novel finding is that the difference between angry and neutral bodies was significant after applying cTBS to either IPL or PMv, both of which have connections to AMG (Clower et al., 2001; Julie Grèzes et al., 2014; Toschi et al., 2017). Previously, cTBS stimulation, limited to pSTS, demonstrated an effect on the BOLD signal in AMG, but only for the condition in which participants viewed dynamic facial stimuli (Pitcher, Japee, Rauth, \& Ungerleider, 2016). That study also explored the influence of stimuli depicting dynamic bodies, but unlike here the stimuli were limited to neutral movements. We find that the application of cTBS created a significantly stronger response to angry compared to neutral bodies, a result that fits well with previous findings of IPL stimulation. Online TMS stimulation of IPL has been shown to result in increased sensitivity to emotional body stimuli (Engelen et al., 2015).

The current findings provide important new insights on the crucial of the AMG with motor related regions in threatening situations, which could be related to action readiness. So far, previous experiments have tried to provide evidence for such interactions by looking at (functional) connectivity between AMG and motor regions. For example, by using DTI, a study by Grèzes et al. (2014) revealed evidence for a pathway between AMG and several cortical motor areas. These findings have further been extended by resting state fMRI studies, in which it was demonstrated that at rest AMG is functionally coupled with sensory and (pre)motor cortices (Roy et al., 2009; Toschi et al., 2017). Likewise, a study exploring psychophysical interactions between the AMG and other brain regions during perception of emotional faces found increased functional coupling between AMG and premotor areas during perception of emotion 62 
faces versus neutral faces (Diano et al., 2017). Specifically the perception of angry versus neutral faces increased functional coupling between AMG and IPL. These studies provide evidence that under threatening circumstances AMG has the option for communication with motor areas through (functional) connectivity, they do not actually show that this is effectively the case. The present study now establishes that, specifically under conditions of perceived social threat, the amygdala is functionally engaging with areas related to action, specifically inferior parietal lobe and ventral premotor cortex.

\section{Accumulating evidence for parietal involvement in emotional body processing}

Following upon previous work establishing a causal role for IPL in emotion body processing (Engelen et al., 2015), we now show that IPL communicates with a number of regions, including $A M G$, to process emotional content of actions. Considering its location and connections, IPL makes for a natural hub where emotion perception is transitioned into an action response. Several fMRI experiments observed emotion specific activation within parietal cortex for both face (Grèzes, Pichon, \& de Gelder, 2007; Kitada, Johnsrude, Kochiyama, \& Lederman, 2010; Sarkheil, Goebel, Schneider, \& Mathiak, 2013) and body stimuli (Goldberg, Christensen, Flash, Giese, \& Malach, 2015; Goldberg, Preminger, \& Malach, 2014; Kana \& Travers, 2012). Previously, an MEG experiment has provided evidence for fast involvement (80-110msec post stimulus onset) of IPL in discriminating between fearful and neutral body postures (Meeren, Hadjikani, Ahlfors, Hämäläinen, \& de Gelder, 2014). Recent work by Mazzoni, Jacobs, Venuti, Silvanto, \& Cattaneo (2017) demonstrated that aIPS holds a representation of affective body movements by using a state-dependent TMS paradigm. Other recent findings in IPL come from a study examining Urbach-Wiethe patients (Hortensius et al., 2017). This patient group suffers from a lesion that is specific to basolateral AMG (BLA). In the experiment, the patient group was presented with face-body compounds that displayed either congruent or incongruent emotions between the face and the body. Irrespective of the emotion represented in the face, IPL showed increased activation if the body expressed fear. Taken together, a clear picture is emerging of the importance of IPL in emotional body processing, and results of the current study demonstrate that this may be implemented through its interactions with AMG.

\section{Recruitment of action observation and execution network for emotion body processing}

Besides AMG, an interaction between stimulation site and valence of the stimulus was also observed in multiple clusters corresponding to areas known for their involvement in the observation and preparation of actions, including clusters in multiple parietal sites, SMA and PM. SMA is an interesting area to consider within the emotional body processing framework, as it has been suggested to act as interface between the limbic system and primary motor cortex (M1) in conveying emotional information. This was demonstrated in a TMS conditioning experiment in which larger MEP amplitude was observed in an emotional visual condition if paired stimulation of SMA and M1 was used, compared to single pulses over M1 (Oliveri et al., 2003). Besides SMA, we also found several parietal clusters that showed an emotion by stimulation site interaction, including bilateral SPL, which is involved in both coordinating visuomotor actions and observation of actions (Culham \& Valyear, 2006) and shows body specific activation as well (Kret et al., 2011). Besides SPL, this interaction was also observed in SPOC and precuneus, of which the former codes for reachable space (Gallivan, Cavina-Pratesi, \& Culham, 2009), and the latter is involved in tasks ranging from visuo-spatial imagery to the experience of agency (Cavanna \& Trimble, 2006). 


\section{Local and remote effects of cTBS}

When investigating the local effects of cTBS stimulation, we found a significant emotion $\mathrm{x}$ session interaction in rPMv. However, neither rPMv, nor rIPL, showed a significant main effect for session. Interestingly, other studies have similarly not found clear decreased local activity following cTBS (see e.g. Ott, Ullsperger, Jocham, Neumann, \& Klein, 2011; van Nuenen, Kuhtz-Buschbeck, Schulz, Bloem, \& Siebner, 2012). This absence of local effects is possibly caused by the fact that TMS effects can be highly variable between participants (Ridding \& Ziemann, 2010), and might this manifest in increased variance in the stimulated areas, rather than an overall suppression (Valchev, Gazzola, Avenanti, \& Keysers, 2016).

In order to uncover any general remote effects of cTBS, we additionally explored the main effect of session in our whole brain analysis. We found several clusters that displayed task invariant changes in the BOLD signal depending on the stimulation condition. Two clusters, namely vmPFC and PCC, showed a cTBS suppression of the BOLD signal compared to baseline irrespective of stimulation site. This suggests that cTBS in general resulted in a suppression of the default mode network (DMN), of which both vmPFC and PCC are parts (Buckner, Andrews-Hanna, \& Schacter, 2008). The DMN generally shows greater activity at rest, and its suppression might result from stronger task engagement after cTBS. However, another important node of the DMN is the PCC, which we also found to have a main effect of session, but suppression in this cluster was specific for IPL stimulation compared to both baseline and PMv stimulation. We also found that stimulation of rPMv suppresses activity in 1SPL, whereas stimulation of rIPL leads to enhanced activity in IPMv. This finding seems to suggest interhemispheric interactions between premotor and parietal areas as a result of stimulation.

In the current experiment we did not include sham cTBS stimulation in the baseline session, which might be seen as a limitation when it comes to the interpretation of stimulation specific results. Including an appropriate control for active TMS stimulation is notoriously difficult, and there is no ideal solution for handling this issue (Duecker \& Sack, 2015). Application of TMS is always accompanied by somatosensory effects and a clicking sound, and in some circumstances (depending on stimulation location and intensity) also peripheral nerve stimulation. Sham stimulation by means of a shielded sham coil provides a credible control for the clicking sound, but is easily distinguishable from active stimulation when it comes to somato-sensation and peripheral nerve stimulation. However, when considering the importance of sham TMS, a distinction needs to be made between paradigms utilizing either online or offline stimulation. While effects induced by somato-sensation and/or peripheral nerve stimulation associated with active stimulation could hinder interpretation of results of online studies, this might be less so for offline paradigms, as these effects are likely of a transient nature (e.g. shorts shifts in arousal or attention). Given that in the current experiment we only included participants that had previous experience with TMS, that sham cTBS in particular lacks credibility compared to active stimulation (due to the lack of distinctively noticeable somatosensory effects and nerve stimulation that is otherwise present), and that our baseline session differed in duration from the other fMRI sessions due to the acquisition of the functional localizers, we opted to not include sham cTBS stimulation. Importantly, we did choose to have two active stimulation sites in addition to the baseline session. In our results we observe, both for the main effect of session as well as the emotion by session interaction, clusters that have different responses for each of the stimulation sites compared to one another, as well as compared to sham. Nonetheless, we cannot rule out that awareness of the participants about lack of stimulation in the baseline session accounts for some of the effects observed in our results. 


\section{Conclusions}

In conclusion, by combining cTBS and fMRI, we were able to establish for the first time a causal relation between brain areas related to action and the perception of affective bodies. We showed specifically how under conditions of perceived social threat, both IPL and PMv dynamically interact with AMG and areas involved in observation and preparation of actions. IPL and PMv are likely candidates to play a key role in preparing fast adaptive action, as is seemingly required by the sight of manifestations of social threat. 


\section{References}

Balconi, M., \& Bortolotti, A. (2013). The "simulation" of the facial expression of emotions in case of short and long stimulus duration. The effect of pre-motor cortex inhibition by rTMS. Brain and Cognition, 83(1), 114-120. https://doi.org/10.1016/j.bandc.2013.07.003

Borgomaneri, S., Vitale, F., \& Avenanti, A. (2015). Early changes in corticospinal excitability when seeing fearful body expressions. Scientific Reports, 5, 14122. https://doi.org/10.1038/srep14122

Borgomaneri, S., Vitale, F., Gazzola, V., \& Avenanti, A. (2015). Seeing fearful body language rapidly freezes the observer's motor cortex. Cortex, 65, 232-245. https://doi.org/10.1016/j.cortex.2015.01.014

Buckner, R. L., Andrews-Hanna, J. R., \& Schacter, D. L. (2008). The Brain 's Default Network: Anatomy , Function, and Relevance to Disease. Ann NY Acad Sci, 1124, 1-38. https://doi.org/10.1196/annals.1440.011

Calbi, M., Angelini, M., Gallese, V., \& Umiltà, M. A. (2017). “Embodied Body Language ”: an electrical neuroimaging study with emotional faces and bodies, 7, 1-12. https://doi.org/10.1038/s41598017-07262-0

Caspers, S., Eickhoff, S. B., Rick, T., Kapri, A. Von, Kuhlen, T., Huang, R., ... Zilles, K. (2011). NeuroImage Probabilistic fibre tract analysis of cytoarchitectonically defined human inferior parietal lobule areas reveals similarities to macaques. NeuroImage, 58(2), 362-380. https://doi.org/10.1016/j.neuroimage.2011.06.027

Cattaneo, L., Sandrini, M., \& Schwarzbach, J. (2010). State-dependent TMS reveals a hierarchical representation of observed acts in the temporal, parietal, and premotor cortices. Cerebral Cortex, 20(9), 2252-2258. https://doi.org/10.1093/cercor/bhp291

Cavanna, A. E., \& Trimble, M. R. (2006). The precuneus: A review of its functional anatomy and behavioural correlates. Brain, 129(3), 564-583. https://doi.org/10.1093/brain/awl004

Clower, D. M., West, R. a, Lynch, J. C., \& Strick, P. L. (2001). The inferior parietal lobule is the target of output from the superior colliculus, hippocampus, and cerebellum. The Journal of Neuroscience, 21(16), 6283-6291. https://doi.org/21/16/6283

Culham, J. C., \& Valyear, K. F. (2006). Human parietal cortex in action. Current Opinion in Neurobiology, 16(2), 205-212. https://doi.org/10.1016/j.conb.2006.03.005

Davis, M., \& Whalen, P. J. (2001). The amygdala : vigilance and emotion. Molecular Psychiatry, 6(1), 13-34.

de Gelder, B., Hortensius, R., \& Tamietto, M. (2012). Attention and awareness each influence amygdala activity for dynamic bodily expressions-a short review. Frontiers in Integrative Neuroscience, 6, 54. https://doi.org/10.3389/fnint.2012.00054

de Gelder, B., Snyder, J., Greve, D., Gerard, G., \& Hadjikhani, N. (2004). Fear fosters flight: a mechanism for fear contagion when perceiving emotion expressed by a whole body. Proceedings of the National Academy of Sciences of the United States of America, 101(47), 16701-6. 
https://doi.org/10.1073/pnas.0407042101

Diano, M., Tamietto, M., Celeghin, A., Weiskrantz, L., Tatu, M., Bagnis, A., ... Cauda, F. (2017). Dynamic Changes in Amygdala Psychophysiological Connectivity Reveal Distinct Neural Networks for Facial Expressions of Basic Emotions. Scientific Reports, 7, 45260. https://doi.org/10.1038/srep45260

Duecker, F., \& Sack, A. T. (2013). Pre-Stimulus Sham TMS Facilitates Target Detection, 8(3), 4-9. https://doi.org/10.1371/journal.pone.0057765

Duecker, F., \& Sack, A. T. (2015). Rethinking the role of sham TMS, 6(February), 1-5. https://doi.org/10.3389/fpsyg.2015.00210

Engelen, T., de Graaf, T. A., Sack, A. T., \& de Gelder, B. (2015). A causal role for inferior parietal lobule in emotion body perception. Cortex, 73, 195-202. https://doi.org/10.1016/j.cortex.2015.08.013

Gallivan, J. P., Cavina-Pratesi, C., \& Culham, J. C. (2009). Is That within Reach? fMRI Reveals That the Human Superior Parieto-Occipital Cortex Encodes Objects Reachable by the Hand. Journal of Neuroscience, 29(14), 4381-4391. https://doi.org/10.1523/JNEUROSCI.0377-09.2009

Goldberg, H., Christensen, A., Flash, T., Giese, M. A., \& Malach, R. (2015). Brain activity correlates with emotional perception induced by dynamic avatars. NeuroImage, 122, 306-317. https://doi.org/10.1016/j.neuroimage.2015.07.056

Goldberg, H., Preminger, S., \& Malach, R. (2014). The emotion-action link? Naturalistic emotional stimuli preferentially activate the human dorsal visual stream. NeuroImage, 84, 254-64. https://doi.org/10.1016/j.neuroimage.2013.08.032

Grèzes, J., Pichon, S., \& de Gelder, B. (2007). Perceiving fear in dynamic body expressions. NeuroImage, 35(2), 959-67. https://doi.org/10.1016/j.neuroimage.2006.11.030

Grèzes, J., Valabrègue, R., Gholipour, B., \& Chevallier, C. (2014). A direct amygdala-motor pathway for emotional displays to influence action: A diffusion tensor imaging study. Human Brain Mapping, 35(12), 5974-5983. https://doi.org/10.1002/hbm.22598

Hadjikhani, N., \& de Gelder, B. (2003). Seeing Fearful Body Expressions Activates the Fusiform Cortex and Amygdala. Current Biology, 13(24), 2201-2205. https://doi.org/10.1016/j.cub.2003.11.049

Hortensius, R., Terburg, D., Morgan, B., Stein, D. J., van Honk, J., \& de Gelder, B. (2017). The Basolateral Amygdalae and Frontotemporal Network Functions for Threat Perception. Eneuro, ENEURO.0314-16.2016. https://doi.org/10.1523/ENEURO.0314-16.2016

Hoshi, E., \& Tanji, J. (2007). Distinctions between dorsal and ventral premotor areas: anatomical connectivity and functional properties. Current Opinion in Neurobiology, 17(2), 234-242. https://doi.org/10.1016/j.conb.2007.02.003

Huang, Y. Z., Edwards, M. J., Rounis, E., Bhatia, K. P., \& Rothwell, J. C. (2005). Theta burst stimulation of the human motor cortex. Neuron, 45(2), 201-206. https://doi.org/10.1016/j.neuron.2004.12.033 
Dynamic interactions between emotion perception and motor preparation networks

Kana, R. K., \& Travers, B. G. (2012). Neural substrates of interpreting actions and emotions from body postures. Social Cognitive and Affective Neuroscience, 7(4), 446-56. https://doi.org/10.1093/scan/nsr022

Kitada, R., Johnsrude, I. S., Kochiyama, T., \& Lederman, S. J. (2010). Brain networks involved in haptic and visual identification of facial expressions of emotion: an fMRI study. NeuroImage, 49(2), 1677 89. https://doi.org/10.1016/j.neuroimage.2009.09.014

Kret, M. E., Pichon, S., Grèzes, J., \& de Gelder, B. (2011). Similarities and differences in perceiving threat from dynamic faces and bodies. An fMRI study. NeuroImage, 54(2), 1755-62. https://doi.org/10.1016/j.neuroimage.2010.08.012

Ledoux, J. E. (2000). Emotion circuits in the brain. Annual Review of Neuroscience, 23(1), 155-184.

Makris, N., Kennedy, D. N., Mcinerney, S., Sorensen, A. G., Wang, R., Caviness, V. S., \& Pandya, D. N. (2005). Segmentation of Subcomponents within the Superior Longitudinal Fascicle in Humans : A Quantitative, In Vivo, DT-MRI Study. Cerebral Cortex, 15(6), 854-869. https://doi.org/10.1093/cercor/bhh186

Mars, R. B., Jbabdi, S., Sallet, J., O’Reilly, J. X., Croxson, P. L., Olivier, E., ... Rushworth, M. F. S. (2011). Diffusion-Weighted Imaging Tractography-Based Parcellation of the Human Parietal Cortex and Comparison with Human and Macaque Resting-State Functional Connectivity. Journal of Neuroscience, 31(11), 4087-4100. https://doi.org/10.1523/JNEUROSCI.5102-10.2011

Mazzoni, N., Jacobs, C., Venuti, P., Silvanto, J., \& Cattaneo, L. (2017). State-dependent TMS reveals representation of affective body movements in the anterior intraparietal cortex. https://doi.org/10.1523/JNEUROSCI.0913-17.2017

Meeren, H. K. M., Hadjikani, N., Ahlfors, S. P., Hämäläinen, M. S., \& de Gelder, B. (2014). Human right parietal cortex shows early preferential responses to naturalistic emotional stimuli: an MEG study. In Society for Neuroscience conference. Washington DC, United States.

Oliveri, M., Babiloni, C., Filippi, M. M., Caltagirone, C., Babiloni, F., Cicinelli, P., ... Rossini, P. M. (2003). Influence of the supplementary motor area on primary motor cortex excitability during movements triggered by neutral or emotionally unpleasant visual cues. Experimental Brain Research, 149(2), 214-221. https://doi.org/10.1007/s00221-002-1346-8

Ott, D. V. M., Ullsperger, M., Jocham, G., Neumann, J., \& Klein, T. A. (2011). Continuous theta-burst stimulation (cTBS) over the lateral prefrontal cortex alters reinforcement learning bias. NeuroImage, 57(2), 617-623. https://doi.org/10.1016/j.neuroimage.2011.04.038

Pessoa, L. (2010). Emotion and Cognition and the Amygdala: from "what is it?" to "what's to be done?" Neuropsychologia, 48(12), 3416-3429.

Picard, N., \& Strick, P. L. (2001). Imaging the premotor areas. Current Opinion in Neurobiology, 11(6), 663672. https://doi.org/10.1016/S0959-4388(01)00266-5

Pichon, S., de Gelder, B., \& Grezes, J. (2008). Emotional modulation of visual and motor areas by 
dynamic body expressions of anger. Social Neuroscience, 3(3-4), 199-212. https://doi.org/10.1080/17470910701394368

Pitcher, D., Japee, S., Rauth, L., \& Ungerleider, L. G. (2016). The superior temporal sulcus is causally connected to the amygdala: A combined TBS-fMRI study, 37(5), 1156-1161. https://doi.org/10.1523/JNEUROSCI.0114-16.2016

Ridding, M. C., \& Ziemann, U. (2010). Determinants of the induction of cortical plasticity by non-invasive brain stimulation in healthy subjects. The Journal of Physiology, 588(13), 2291-2304. https://doi.org/10.1113/jphysiol.2010.190314

Rizzolatti, G., Fogassi, L., \& Gallese, V. (2002). Motor and cognitive functions of the ventral premotor cortex. Current Opinion in Neurobiology, 12(2), 149-154. https://doi.org/10.1016/S09594388(02)00308-2

Roy, A. K., Shehzad, Z., Margulies, D. S., Kelly, A. M. C., Uddin, L. Q., Gotimer, K., ... Milham, M. P. (2009). Functional connectivity of the human amygdala using resting state fMRI. NeuroImage, 45(2), 614-626. https://doi.org/10.1016/j.neuroimage.2008.11.030

Sarkheil, P., Goebel, R., Schneider, F., \& Mathiak, K. (2013). Emotion unfolded by motion: A role for parietal lobe in decoding dynamic facial expressions. Social Cognitive and Affective Neuroscience, 8(8), 950-957. https://doi.org/10.1093/scan/nss092

Toschi, N., Duggento, A., \& Passamonti, L. (2017). Functional Connectivity in AmygdalarSensory/(Pre)Motor networks at rest: New evidence from the Human Connectome Project. European Journal of Neuroscience, 45(9), 1224-1229. https://doi.org/10.1111/ejn.13544

Tremblay, P., Sato, M., \& Small, S. L. (2012). Neuropsychologia TMS-induced modulation of action sentence priming in the ventral premotor cortex. Neuropsychologia, 50(2), 319-326. https://doi.org/10.1016/j.neuropsychologia.2011.12.002

Urgesi, C., Calvo-Merino, B., Haggard, P., \& Aglioti, S. M. (2007). Transcranial magnetic stimulation reveals two cortical pathways for visual body processing. The Journal of Neuroscience, 27(30), 80238030. https://doi.org/10.1523/JNEUROSCI.0789-07.2007

Urgesi, C., Candidi, M., Ionta, S., \& Aglioti, S. M. (2007). Representation of body identity and body actions in extrastriate body area and ventral premotor cortex. Nature Neuroscience, 10(1), 30-31. https://doi.org/10.1038/nn1815

Valchev, N., Gazzola, V., Avenanti, A., \& Keysers, C. (2016). Primary somatosensory contribution to action observation brain activity-combining fMRI and cTBS. Social Cognitive and Affective Neuroscience, 11(8), 1205-1217. https://doi.org/10.1093/scan/nsw029

van Nuenen, B. F. L., Kuhtz-Buschbeck, J., Schulz, C., Bloem, B. R., \& Siebner, H. R. (2012). WeightSpecific Anticipatory Coding of Grip Force in Human Dorsal Premotor Cortex. Journal of Neuroscience, 32(15), 5272-5283. https://doi.org/10.1523/JNEUROSCI.5673-11.2012 



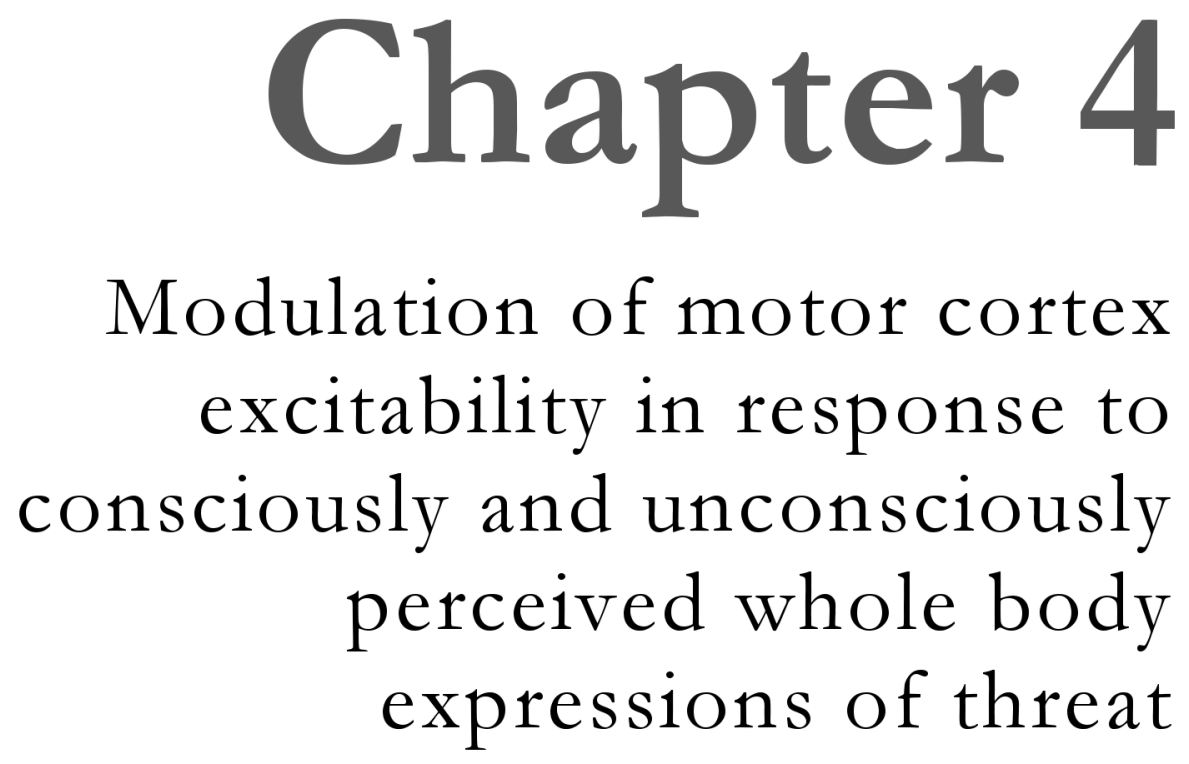

Based on:

Engelen, T., Zhan, M., Sack, A.T., \& de Gelder, B. (in preparation). Modulation of motor cortex excitability in response to consciously and unconsciously perceived whole body ex-pressions of threat. 



\section{Abstract}

The observation of threat emotions in others are strong cues for triggering an action response. One method of capturing such action responses is by measuring the amplitude of Motor Evoked Potentials (MEPs) elicited with single pulse TMS over the primary motor cortex. Indeed, it has been shown that viewing whole body expressions of threat modulate the size of MEP amplitude. Likewise, emotional cues have been shown to act on certain brain areas even outside of conscious awareness. In the current study, we wanted to explore if the influence of viewing whole body expressions of threat extend to stimuli that are presented outside of conscious awareness. In order to accomplish this, we combined the measurement of MEPs with a continuous flash suppression task. In experiment 1, participants were presented with images of neutral bodies, fearful bodies, or objects that were either perceived consciously or unconsciously, while single pulses of TMS were applied at different times after stimulus onset $(200 \mathrm{~ms}$, $500 \mathrm{~ms}$ or $700 \mathrm{~ms}$ ). In experiment 2 stimuli consisted of neutral bodies, angry bodies or objects, and pulses were applied at either $200 \mathrm{~ms}$ or $400 \mathrm{~ms}$ post stimulus onset. In experiment 1 , there was a general effect of the time of stimulation, but no clear condition specific effects were evident. In experiment 2 there was a significant difference between angry bodies and objects at $200 \mathrm{~ms}$, but only when they were consciously perceived by the participant. Future studies are needed looking into earlier effects of MEP modulation by emotion body stimuli, as well as an exploration of other outcome measures such as intracortical facilitation.

\section{Introduction}

Perceiving threat cues from others will likely trigger a fight, flight or freeze response in the observer supporting the notion that emotion and action readiness are tightly linked (Eder \& Rothermund, 2013; Frijda, 2010). This action readiness is reflected in physiological responses such as changes in heart rate, pupil dilation, skin conductance and muscle activations (Bradley, Miccoli, Escrig, \& Lang, 2008; Huis In 't Veld, Van Boxtel, \& de Gelder, 2014; Roelofs, Hagenaars, \& Stins, 2010), and is also evident in the state of the cortical motor system (e.g. de Gelder, Snyder, Greve, Gerard, \& Hadjikhani, 2004; Goldberg, Preminger, \& Malach, 2014). The current study set out to explore if and when stimuli portraying whole body postures of emotion alter motor cortex excitability, and whether conscious perception of such stimuli influences this modulation.

\section{Action readiness in response to social threat}

One direct way of assessing the state of the motor system is by evaluating corticospinal excitability (CSE), which can be measured by calculating the amplitude of motor evoked potentials (MEPs). MEPs are elicited with single pulses of transcranial magnetic stimulation (TMS), and they can be measured from the targeted muscle using electromyography (EMG). Emotion related modulations of MEP amplitude were already demonstrated in response to emotional scenes. Studies examining responses to either pleasant or unpleasant pictures taken from the International Affective Picture System (IAPS) have shown increased MEP amplitude for 
affective versus neutral scenes (Coelho, Lipp, Marinovic, Wallis, \& Riek, 2010; Coombes et al., 2009; Hajcak et al., 2007; van Loon, van den Wildenberg, van Stegeren, Ridderinkhof, \& Hajcak, 2010). Likewise, modulation of MEP amplitude in response to social threat has been demonstrated by comparing the response to fearful faces to either neutral or happy faces. Again, an increase in MEP amplitude in response to fearful faces compared to both other conditions was observed (Schutter, Hofman, \& Van Honk, 2008).

Similarly, studies have also explored emotion-dependent MEP modulations in response to whole body expressions of emotion, however with mixed results. Initial work showed that at $300 \mathrm{~ms}$ post stimulus onset, both pictures of emotional (joy or fear) and neutral movements elicited greater MEP amplitude compared to neutral static postures, suggesting in this case it might be implied motion, rather than valence, modulating MEP amplitude (Borgomaneri, Gazzola, \& Avenanti, 2012). On the other hand, not only implied motion, but also social intentions seem to be of importance. Bucchioni, Cavallo, Ippolito, Marton, \& Castiello (2013) showed for example that MEP amplitude is enhanced when observing an actor passing a ball to another actor, rather than throwing the ball at a wall, suggesting the motor system certainly codes for more than simply the amount of perceived motion within an action. Recent work by Hortensius, de Gelder, \& Schutter (2016) found increased MEP amplitude in response to dynamic angry whole body movements at $300 \mathrm{~ms}$ post stimulus onset compared to fearful and neutral movements, irrespective of whether the direction of the movement was towards or away from the observer. Further experiments looking at whole body expressions of emotion have shown an increase in MEP amplitude in response to fearful compared to neutral actions as early as 70-90ms post stimulus onset (Borgomaneri, Vitale, \& Avenanti, 2015). Yet, another experiment found a reduction in MEP amplitude of emotional versus neutral bodies at $150 \mathrm{~ms}$, whereas a general increase in MEP amplitude for all stimuli was observed at $300 \mathrm{~ms}$ (Borgomaneri, Gazzola, \& Avenanti, 2014). When also taking into account intracortical facilitation (ICF) and inhibition (ICI), it was found that at $125 \mathrm{~ms}$ post stimulus onset ICF was suppressed specifically for fearful bodies, whereas this effect was absent for both MEPs and ICI (Borgomaneri, Vitale, Gazzola, \& Avenanti, 2015). This early suppression effect of ICF in response to fearful bodies was later replicated, and found to be related to individual Behavioral Inhibition System (BIS) sensitivity (Borgomaneri, Vitale, \& Avenanti, 2017). The current study aimed to clarify whether MEPs are indeed modulated by emotional body expressions by assessing MEP amplitude at several time points after stimulus onset in response to both fearful and angry whole-body expressions of emotion.

\section{Does unconscious processing of social threat trigger action readiness?}

The aforementioned studies demonstrate that motor cortex excitability can be modulated by the presence of social threat represented by whole body expressions of emotion. So far, experiments have focused on MEP modulation in response to consciously perceived threat. However, research suggests that salient signals, such as threat, have the ability to be processed also when presented outside of visual awareness. 
Ample evidence on the possibility of unconscious processing of emotions results from the study of a phenomenon called affective blindsight. Affective blindsight can occur in patients that are cortically blind as a result of lesions to their primary visual cortex. Despite being blind in part of their visual field, they are still capable of judging in that blind visual field whether a face is expressing happy or fear above chance level, without having any conscious percept of the stimulus (de Gelder, Vroomen, Pourtois, \& Weiskrantz, 1999). Unconscious processing of emotional information is not unique to these patients however. By applying TMS to primary visual cortex to disrupt visual awareness, Jolij \& Lamme (2005) were able to mimic affective blindsight in healthy participants.

Importantly, processing of unconscious emotional information has an effect on the observer, both on a physiological and a behavioural level. On the physiological level, unconsciously perceived emotions have been shown to cause changes in skin conductance (Esteves, Dimberg, \& Öhman, 1994; Williams et al., 2004), pupil dilation (Tamietto et al., 2009, 2015), heart rate (Ruiz-Padial, Mata, Rodríguez, Fernández, \& Vila, 2005; Ruiz-Padial, Vila, \& Thayer, 2011), evoking of startle reflexes (Hamm et al., 2003), levels of stress hormone (Van Honk et al., 1998), and have even been shown to change EMG responses measured from facial muscles in both patients (Tamietto et al., 2009) and healthy participants (Dimberg, Thunberg, \& Elmehed, 2000; Tamietto \& de Gelder, 2008b). On the behavioural level, masked fearful faces have been shown to modulate orienting of covert spatial attention (Carlson \& Reinke, 2008), the recognition of unmasked happy faces gets delayed by simultaneously presented masked fearful face (Tamietto \& de Gelder, 2008a), and suppressed angry faces can influence the likeability of novel items (Almeida, Pajtas, Mahon, Nakayama, \& Caramazza, 2013), to name a few major examples.

Many studies have looked into the level of processing of unconsciously perceived emotion in the brain, many of which have demonstrated the crucial role of subcortical areas. Subcortical areas that have been highlighted in multiple studies include superior colliculus, amygdala, and pulvinar (Tamietto \& de Gelder, 2010). However, cortical regions have also been implicated as playing a role in processing of unconscious emotional signals. For example, one fMRI study involving a patient suffering from visual extinction, following lesions in the parietal cortex, found that presenting fearful body postures in the affected hemifield resulted in activations in striate and extrastriate visual areas, as well as activity in posterior cingulate cortex (Tamietto et al., 2015). Another fMRI study measured responses to angry and neutral whole body expressions presented in the blind field of a cortically blind patient. When contrasting unconsciously perceived angry and neutral bodies, they found widespread cortical activity, which included somatosensory, motor and premotor cortices (Van den Stock et al., 2011).

In general, similar to consciously perceived threat, it seems that unconsciously perceived threat might relate to action preparation in a similar way, as is suggested by for example changes in heart rate (Ruiz-Padial et al., 2005, 2011), or findings of motor cortex activity in response to angry bodies (Van den Stock et al., 2011). The question remains, however, if these unconscious brain responses to social threat extend to changes in excitability of the motor system, which provide a direct measure of action preparation. In addition, previously used methodology to study this topic (fMRI and most physiological measures), lack the ability to draw any conclusions about timing of such preparatory responses. This current study therefore aimed to answer the question whether unconsciously perceived whole body expressions of threat can also prime action readiness in M1, and the timing of when such excitability changes would occur. In order to accomplish this, we employed the continuous flash suppression (CFS) paradigm (Tsuchiya 
\& Koch, 2005). CFS is a method whereby stimuli are rendered invisible to the participant by suppression of one image through interocular competition. Suppression is accomplished by presenting one eye with a dynamic colourful mask at a $10 \mathrm{~Hz}$ flickering frequency, while the other eye is presented with a stimulus of interest, but with lower contrast. The result is a reliably suppression of the conscious percept of the stimulus of interest.

A previous breaking from continuous flash suppressions (b-CFS) experiment established that suppression times for body stimuli were shorter for angry compared to both neutral and fearful postures, whereas fearful postures had longer suppression times compared to both other categories (Zhan, Hortensius, \& de Gelder, 2015). Despite fear and anger both conveying signals of threat, neuroimaging results suggest they might not be processed in the exact same way, as threat conveyed by an anger signal is more direct and less ambiguous than fear (Pichon, de Gelder, \& Grèzes, 2009). Therefore, we decided to explore both the effects of fear and angry body postures on motor cortex excitability.

Thus far, no previous studies have looked into modulation of MEPs in response to unconsciously perceived emotion stimuli. Feasibility of inducing changes in motor cortex excitability through masked stimuli has been demonstrated (Théoret, Halligan, Kobayashi, Merabet, \& Pascual-Leone, 2004), but to date very few studies have used MEP amplitude as an outcome measure combined with presentations of stimuli outside of awareness. One study assessed MEP amplitude in response to masked self-images, and found a significant increase in MEP amplitude in response to self- compared to other-faces (Théoret, Kobayashi, et al., 2004). On the other hand, a study using a masked priming paradigm that investigated the effect of implied action images of hands found results for action versus still hands, but only when the stimuli were presented supraliminally (Mattiassi, Mele, Ticini, \& Urgesi, 2014). Although action stimuli in general have been shown to modulate CSE (Fadiga, Craighero, \& Olivier, 2005), they do not carry the same saliency and relevance as emotionally laden actions, which could explain why their influence on M1 is restricted to conscious perception.

The goal of the current experiment was twofold; firstly, we aimed to replicate previous findings that consciously perceived, threatening body postures, compared to neutral postures, may alter the state of the motor cortex. Secondly, we wanted to explore whether such modulations were also present in unconscious perception of threatening body postures. In experiment 1, participants performed a CFS task in which they had to indicate after each trial whether a stimulus was consciously perceived or not. The stimulus categories presented were either fear bodies, neutral bodies (standing still), or objects (lamps). In order to manipulate stimulus visibility images were presented to either the suppressed eye only, or both eyes (seen or unseen). During task performance, single TMS pulses were administered to the hand hot-spot of left M1 while MEPs were measured from the first dorsal interosseous (FDI) muscle of the right hand. TMS pulses were triggered at three different time points, either at 200,500 or $700 \mathrm{~ms}$ post stimulus onset. The design of experiment 2 was similar to that of experiment 1, with the exception that stimulus categories consisted of either angry bodies, neutral bodies, or objects, while TMS pulses were triggered at either 200 or $400 \mathrm{~ms}$ post stimulus onset. We hypothesized that changes of MEP amplitude would be specific to the stimulus categories portraying threat (fear and anger), and to the early time points, both in the consciously and unconsciously perceived threat situations. As unconsciously perceived emotion has been shown to have faster effects on for example EMG or EEG measures (Tamietto et al., 2009; Williams et al., 2004), we might also observe effects for unseen threat stimuli in an earlier time window, compared to the seen stimuli. 


\section{Methods}

\section{Participants}

Twenty-one healthy volunteers (15 female, mean age $(\mathrm{SD})=24.5(3.3))$ participated in experiment 1 , and thirty healthy volunteers (23 female, mean age (SD) $=21(3.2)$ ) participated in experiment 2 . All participants were right handed and had normal or corrected to normal vision. If a participant had corrected to normal vision, they were asked to wear contact lenses during the experiment to allow for the wearing of the prism glasses (see task). All participants were unaware of the goal of the study until after the completion of the experiment. Before the start of the experimental proceedings, participants provided written informed consent and were screened for TMS safety based on published safety guidelines (Rossi, Hallett, Rossini, \& Pascual-Leone, 2012). The study was performed in accordance to the Declaration of Helsinki and approved by the local ethical committee.

\section{Stimuli}

Stimuli consisted of static, gray-scaled body images portraying either neutral or fearful postures in experiment 1 , or neutral and angry postures in experiment 2 . These postures were portrayed by both male and female actors, with their facial information removed. In total 10 actor identities ( 5 females) were used. As a control condition gray-scale images of lamps were used (Stein, Sterzer, \& Peelen, 2012). The stimuli spanned within $1.8^{\circ} \times 4.3^{\circ}$ visual angles.

\section{Continuous Flash Suppression task}

During the experiment, the participants performed a CFS task for which the parameters were as follows; the task was presented with MATLAB (MathWorks, Natick, MA, USA) using Psychtoolbox. The task background was set to gray $(\mathrm{RGB}$ value $=128,128,128)$, on which two rectangles $\left(240 \times 160\right.$ pixels, $6.4^{\circ} \times 4.27^{\circ}$ visual angle) were placed side by side at the center of the screen, 286 pixels apart from one another (visual angle $=6.67^{\circ}$ ), and with a black fixation cross in the center of each rectangle. A 10-pixel frame delineated the border of the rectangles. To allow for the presentation of different stimuli to each of the eyes, participants wore prism glasses (diopter $=8$ ), ensuring that the perception of each rectangle was shifted back to the center of the screen (method as descried in Schurger, 2009). A cardboard divider was placed between the screen and the chinrest, dividing the screen into two halves, which ensured that each eye would not receive information from the contralateral side of the screen.

During each trial, dynamic noise images $(160 \times 240$ pixels, flashing at $10 \mathrm{~Hz})$ were presented in one of the two rectangular frames. The noise images, consisting of overlapping and colorful small rectangles (height and width within $2^{\circ}$ ), were drawn randomly from 600 unique noise images. The target stimulus was projected into the other rectangular frame. For the seen trials the target stimulus was overlaid on the noise images, and thus was presented to both eyes. The duration of the stimulus presentation was set to $1.5 \mathrm{~s}$, and consisted of gradually ramping up of the stimulus contrast from $0 \%$ to $50 \%$ contrast in the first $500 \mathrm{~ms}$, then presentation of the stimulus at $50 \%$ contrast for another $500 \mathrm{~ms}$, and lastly ramping down stimulus contrast back to $0 \%$. The dynamic noise images were presented for 2 more seconds after the offset of the target stimulus, to ensure no afterimage of the target was perceived. At the end of each trial a response screen $(2 \mathrm{~s})$ was presented, during which the participant had to respond with their left hand whether a stimulus (body or lamp) had been perceived or not. To rule out any possible 
contamination of the EMG signal in the right hand caused by response preparation in contralateral motor cortex, the two answers keys presented in the response screen were randomly switched in a counterbalanced manner, and participants were instructed to not respond until the response screen appeared. Trials in which the response fell outside of the $2 \mathrm{~s}$ response window were excluded from analysis. A jittered ISI of 2.5, 3, 3.5, 4, or $4.5 \mathrm{~s}$ followed the offset of the response window.

\section{TMS stimulation and EMG recording}

During the performance of the CFS task, single pulse TMS (spTMS) was applied over the M1 hand hotspot in the left hemisphere at $120 \%$ resting motor threshold (mean stimulation intensity $45 \%$ MSO in experiment 1 , and $42 \%$ MSO in experiment 2 ) using a MC-B70 figure-ofeight coil and Magpro X100 stimulator (Medtronic Functional Diagnostics A/S, Skovlunde, Denmark). The coil was placed tangential to the scalp with the handle pointing backwards at an angle of 45 degrees from the midline. Motor threshold of each participant was determined as the lowest stimulation intensity needed to evoke an MEP $(>0.05 \mathrm{mV})$ in five out of ten pulses, while the right hand was at rest. For both experiments, the inter-pulse interval was jittered around 9 seconds, and the timing of the pulse was either 200,500 or $700 \mathrm{~ms}$ post stimulus onset in experiment 1 , and 200 or $400 \mathrm{~ms}$ in experiment 2 . One pulse was applied randomly at one of the timings per trial. EMG of the right FDI muscle was recorded for $100 \mathrm{~ms}$ pre- until $150 \mathrm{~ms}$ post TMS pulse. Pre-gelled silver-chloride disposable surface electrodes were placed in a belly-tendon montage with a ground electrode on the wrist. The EMG signal was recorded using a Powerlab 4/35 data acquisition device with a Bio Amp system (ADInstruments, Sydney, Australia). The signal was amplified, sampled at $4 \mathrm{k} / \mathrm{s}$, band-pass filtered $(20-2000 \mathrm{~Hz})$, digitized and stored on a computer for offline analysis.

\section{Procedure}

Each participant came in for one 2-hour session. Participants were seated in front of a computer screen used for presentation of the task (liyama prolite B2481HS, 24", resolution 1920 $x$ 1080). First, each participant had a short practice session to ensure they could stably merge the two rectangles, and that suppression of the stimuli was successful. Electrodes for the EMG recordings were placed on the right hand, and it was checked that noise levels were below $0.02 \mathrm{mV}$. An optimal location for stimulation was found by moving the TMS coil over left motor cortex and observing the peak-to-peak amplitudes of MEPs. Once a location was found that elicited the largest peak-to-peak MEP amplitude for the stimulation of the FDI muscle, the coil was fixed into position using a coil holder, while the participant rested their head in a chinrest. Participants were instructed to sit as still as possible during the rest of the session. To ensure that no shifts in coil position occurred, the MEP amplitudes were monitored throughout the session and adjustments were made by the experimenter if necessary. In experiment 2 , Neuronavigation was used and the location of the hotspot was marked on a template brain to ensure more stability of the stimulation site (BrainVoyager TMS Neuronavigator software, Brain Innovation, Maastricht, The Netherlands). During each run participants were asked to keep their right hand as relaxed as possible. They were additionally instructed to fixate and free-fuse the two rectangles into one. The experiment only started after participants reported that they could clearly see only one rectangle, and that this view was stable. All participants reported successful and stable fusion. A total of 4 runs of the task were performed, each run containing 90 trials and lasting around 13 minutes. A total of 360 trials were acquired for each 
participant, half of which were seen and half of which were unseen. In experiment 1 , for each of the 18 conditions ((2 visibility(seen/unseen) x 3 stimulus (body fear/body neutral/lamp) x 3 TMS timing $(200 \mathrm{~ms} / 500 \mathrm{~ms} / 700 \mathrm{~ms})) 20$ trials were presented. In experiment 2 , each of the 12 conditions ((2 visibility (seen/unseen) x 3 stimulus (body anger/body neutral/lamp) x TMS timing $(200 \mathrm{~ms} / 400 \mathrm{~ms})$ were presented for 30 trials.

\section{Data pre-processing}

Neurophysiological and behavioral data were processed offline. All trials that were incorrect (i.e. unseen trials that were reported as seen and vice versa) were removed from analysis to ensure only successfully suppressed trials were included $(12.3 \%$ of trials in experiment $1,12.6 \%$ of trials in experiment 2). Mean MEP peak-to-peak values were calculated in $\mathrm{mV}$ and outliers were removed that deviated more than 2.5 SDs from the mean of each condition $(1.7 \%$ of trials in experiment $1,2.1 \%$ of trials in experiment 2$)$. To rule out any effects of muscle precontracting on MEP amplitude, the highest peak-to-peak value from the $100 \mathrm{~ms}$ preceding the TMS pulse were used to exclude all trials with a deviation of more than 3 SDs of the mean in each run, as the noise on the EMG signal could sometimes vary between runs $(1.6 \%$ of trials in experiment $1,1.7 \%$ of trials in experiment 2 ).

\section{Analysis}

For experiment 1 , a $2 \times 3 \times 3$ repeated-measures (RM) ANOVA was used to investigate the effects of stimulus visibility (seen/unseen) of different stimulus conditions (body fear/body neutral/lamp) on MEP amplitude at three different time points $(200 \mathrm{~ms} / 500 \mathrm{~ms} / 700 \mathrm{~ms})$. For experiment 2, a $2 \times 3 \times 2$ RM ANOVA was used to investigate the effects of stimulus visibility (seen/unseen) of different stimulus categories (body angry, body neutral/lamp) on MEP amplitude at two different time points $(200 \mathrm{~ms} / 400 \mathrm{~ms})$.

\section{Results}

\section{Results experiment 1}

A $2 \times 3 \times 3$ RM ANOVA showed a significant main effect of the time of the pulse $(F(2,40)=$ $\left.8.639, p=.001, \eta_{\mathrm{p}}^{2}=.302\right)$, as well as a trend for a visibility $\mathrm{x}$ stimulus condition $\mathrm{x}$ pulse time interaction $\left(F(4,80)=2.276, p=.068, \eta_{\mathrm{p}}^{2}=.102\right)$. There was no main effect of visibility $(F(1,20)$ $\left.=1.855, p=.188, \eta_{\mathrm{p}}^{2}=.085\right)$, nor a main effect of stimulus condition $(F(2,40)=0.645, p=$ $\left..530, \eta_{\mathrm{p}}^{2}=.031\right)$, nor any interactions between either visibility and stimulus condition $(F(2,40)$ $\left.=0.743, p=.482, \eta_{\mathrm{p}}^{2}=.036\right)$, or stimulus condition and pulse time $(F(2,40)=1.267, p=.290$, $\left.\eta_{\mathrm{p}}^{2}=.060\right)$.

To follow up on the trend for the three-way interaction, we first ran two separate RM ANOVAs for each of the visibility conditions. In the seen data there was a significant main effect of pulse time $\left(F(2,40)=9.231, p=.001, \eta_{\mathrm{p}}^{2}=.316\right)$, which was a trend in the unseen data $(F(2,40)$ $\left.=2.733, p=.077, \eta_{\mathrm{p}}^{2}=.120\right)$. Neither seen nor unseen data had a main effect of stimulus condition $\left(F(2,40)=0.812, p=.451, \eta_{\mathrm{p}}^{2}=.039\right.$ for unseen, $F(2,40)=0.356, p=.703, \eta_{\mathrm{p}}^{2}=$ .017 for seen). In the unseen data, there was additionally a trend for an interaction between 
stimulus condition and pulse time $\left(F(4,80)=2.357, p=.061, \eta_{\mathrm{p}}^{2}=.105\right)$, which was absent in the seen trials $\left(F(4,80)=0.453, p=.770, \eta_{\mathrm{p}}^{2}=.022\right)$.

To disentangle the stimulus condition x pulse time interaction in the unseen trials, we looked at each pulse time separately for an effect of stimulus condition. At $200 \mathrm{~ms}$, there was a trend for an effect of stimulus condition $\left(F(2,40)=2.840, p=.070, \eta_{\mathrm{p}}^{2}=.124\right)$, caused by a trend for a difference between the neutral body and lamp condition $(p=.070)$, which was absent between the fear body and neutral body condition $(p=1)$, and between the fear body and lamp condition $(p=.141)$. There was no significant effect of condition at either $500 \mathrm{~ms}(\mathrm{~F}(2,40)=$ $\left.1.455, p=.245, \eta_{\mathrm{p}}^{2}=.068\right)$, or $700 \mathrm{~ms}\left(\mathrm{~F}(2,40)=1.589, p=1.589, \eta_{\mathrm{p}}^{2}=.074\right)$. See figure 1 and table 1 for a full overview of the results of experiment 1 .

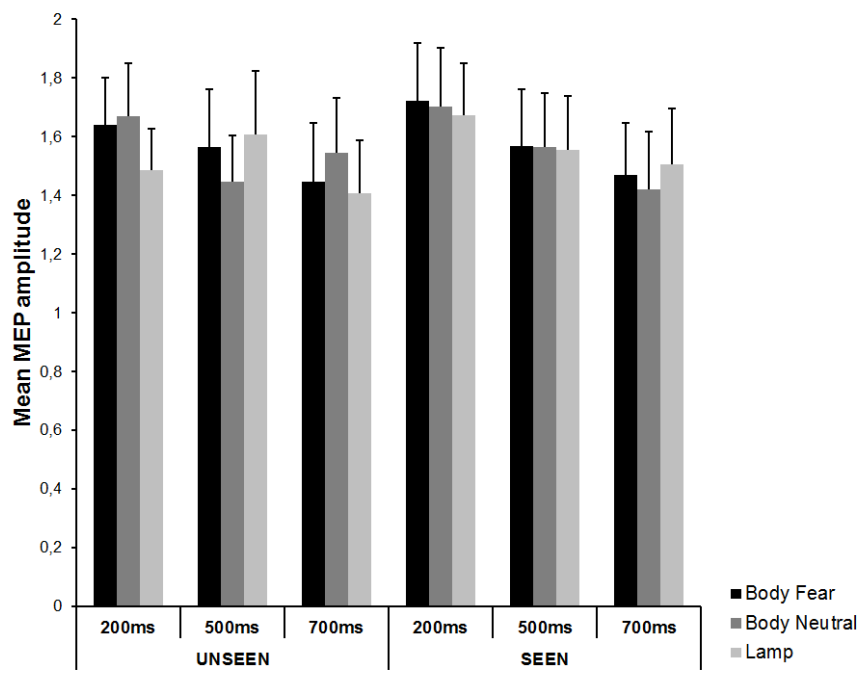

Figure 2. Mean MEP amplitude for the different conditions in experiment 1.

Table 1. Mean MEP amplitude (SE) for the different condition in experiment 1.

\begin{tabular}{|c|c|c|c|c|c|c|}
\hline & UNSEEN & & & SEEN & & \\
\hline & $200 \mathrm{~ms}$ & $500 \mathrm{~ms}$ & $700 \mathrm{~ms}$ & $200 \mathrm{~ms}$ & $500 \mathrm{~ms}$ & $700 \mathrm{~ms}$ \\
\hline Body fear & $1,64( \pm 0,16)$ & $1,57( \pm 0,19)$ & $1,45( \pm 0,20)$ & $1,72( \pm 0,20)$ & $1,57( \pm 0,20)$ & $1,47( \pm 0,18)$ \\
\hline Body neutral & $1,67( \pm 0,18)$ & $1,45( \pm 0,16)$ & $1,55( \pm 0,19)$ & $1,70( \pm 0,20)$ & $1,57( \pm 0,18)$ & $1,42( \pm 0,20)$ \\
\hline Lamp & $1,49( \pm 0,14)$ & $1,61( \pm 0,22)$ & $1,41( \pm 0,18)$ & $1,67( \pm 0,18)$ & $1,55( \pm 0,18)$ & $1,51( \pm 0,19)$ \\
\hline
\end{tabular}


A $2 \times 3 \times 2$ RM ANOVA showed no main effects for either visibility $(F(1,29)=2.289, p=.141$, $\left.\eta_{\mathrm{p}}^{2}=.073\right)$, stimulus condition $\left(F(2,58)=0.448, p=.641, \eta_{\mathrm{p}}^{2}=.015\right)$, or pulse time $(F(1,29)=$ $\left.1.281, p=.267, \eta_{\mathrm{p}}^{2}=.042\right)$. There were also no interactions between either visibility and condition $\left(F(2,58)=1.942, p=.153, \eta_{\mathrm{p}}^{2}=.063\right)$, visibility and pulse time $(F(1,29)=0.361, p=$ $\left..553, \eta_{\mathrm{p}}^{2}=.012\right)$, condition and pulse time $\left(F(2,58)=.329, p=.721, \eta_{\mathrm{p}}^{2}=.011\right)$, or a visibility $\mathrm{x}$ stimulus condition $\mathrm{x}$ pulse time interaction $\left(F(2,58)=0.372, p=.691, \eta_{\mathrm{p}}^{2}=.013\right)$.

As we had the a priori hypothesis that modulations of MEP amplitude would occur specifically at an early time point in the seen condition, we additionally ran two RM ANOVAs in which we, per visibility condition, check for the effect of stimulus condition at $200 \mathrm{~ms}$. There was no significant effect of stimulus condition in the unseen condition $\left(F(2,58)=0.381, p=.685, \eta^{2} \mathrm{p}\right.$ $=.013)$, but there was a significant effect of stimulus condition in the seen condition $(F(2,58)$ $\left.=3.358, p=.042, \eta_{\mathrm{p}}^{2}=.104\right)$, which was caused by a significant difference between angry bodies and lamps $(p=.040)$, with a higher MEP amplitude for angry bodies compared to lamps $($ mean $(\mathrm{SE})=1.36(0.13)$ and $1.23(0.11)$, respectively). However, the hypothesized difference between neutral and angry bodies did not reach significance $(p=.361)$.

For an overview of the results of experiment 2 see figure 2 and table 2.

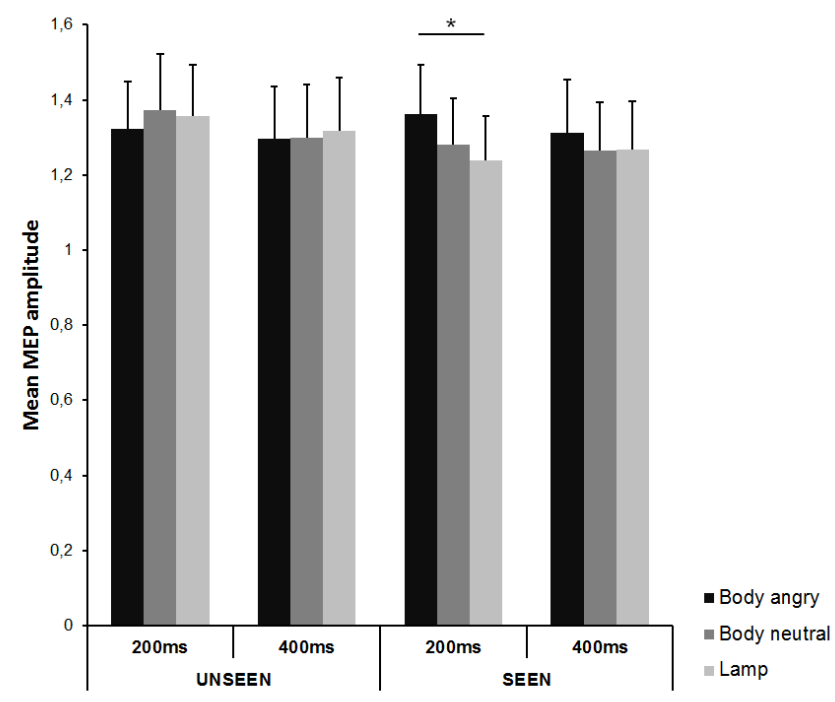

Figure 3. Mean MEP amplitude for the different conditions in experiment 2. 
Table 2. Mean MEP amplitude (SE) for the different conditions in experiment 2.

\begin{tabular}{|c|c|c|c|c|}
\hline & UNSEEN & & SEEN & \\
\hline & $200 \mathrm{~ms}$ & $400 \mathrm{~ms}$ & $200 \mathrm{~ms}$ & $400 \mathrm{~ms}$ \\
\hline Body angry & $1,32( \pm 0,12)$ & $1,30( \pm 0,14)$ & $1,36( \pm 0,13)$ & $1,31( \pm 0,14)$ \\
\hline Body neutral & $1,37( \pm 0,15)$ & $1,30( \pm 0,14)$ & $1,28( \pm 0,12)$ & $1,26( \pm 0,13)$ \\
\hline Lamp & $1,36( \pm 0,14)$ & $1,32( \pm 0,14)$ & $1,24( \pm 0,12)$ & $1,27( \pm 0,13)$ \\
\hline
\end{tabular}

\section{Discussion}

The current experiment set out to explore whether viewing emotional body postures can modulate the excitability of motor cortex during both conscious and unconscious perception. Specifically, in experiment 1 we compared the amplitude of MEPs for conscious and unconscious viewing of either neutral or fearful bodies, and lamps as a control stimuli, at different time points after stimulus onset $(200,500$ and $700 \mathrm{~ms})$ in a continuous flash suppression task. Whereas there seemed to be a general modulation of MEP amplitude depending on the time point of the stimulation, no clear effect of the presented stimulus was apparent when the stimulus was consciously perceived. In the unconscious condition, only at $200 \mathrm{~ms}$ was there a significant effect of stimulus condition, with a trend for a difference between the neutral body and the lamp condition. In experiment 2, we again compared MEP amplitudes in responses to consciously or unconsciously perceived bodies and lamps, this time investigating the effect of anger, and focusing on the time points $200 \mathrm{~ms}$ and $400 \mathrm{~ms}$ post stimulus onset. Only at $200 \mathrm{~ms}$ in the seen conditions was there a significant effect that resulted from greater MEP amplitude for angry bodies compared to lamps, but not to neutral bodies. Together the findings of both experiments suggest that body stimuli in general can elicit an increase in motor cortex excitability at early time points, both when perceived consciously and unconsciously. However, the effects observed in these experiments are weak, and no significant differences were found between neutral and emotional bodies. Below we will discuss several factors that might have contributed to this.

\section{Not only the where, but also the when of action preparation}

A main advantage of TMS is its high temporal resolution, and using this method to study motor cortex reactivity to threat can give valuable insight into the exact timing of preparatory responses. So far, several studies have tried to address this question. Their results suggest that these responses can occur already at very early stages of processing. Significant differences in motor cortex excitability between emotional and neutral body postures have been shown to occur as early as 70-90ms post stimulus onset (Borgomaneri, Vitale, \& Avenanti, 2015), but also at 100-125ms (Borgomaneri et al., 2017; Borgomaneri, Vitale, Gazzola, et al., 2015), and $150 \mathrm{~ms}$ (Borgomaneri et al., 2014). These findings are in line with MEG results showing emotion specific effects for body stimuli in the dorsal stream for action as early as $80 \mathrm{~ms}$ post stimulus onset (Meeren, Hadjikhani, Ahlfors, Hämäläinen, \& de Gelder, 2016). When looking at later time points, specifically $300 \mathrm{~ms}$, effects seem to be driven by actions in general rather than being emotion specific (Borgomaneri et al., 2012). On the other hand, Hortensius et al. (2016) 
found an effect of anger compared to both fear and neutral at $300 \mathrm{~ms}$. In the current study, we used the time points $200 \mathrm{~ms}, 400 \mathrm{~ms}, 500 \mathrm{~ms}$, and $700 \mathrm{~ms}$. In light of previous work, there is a possibility that our lack of clear findings is the result of 'missing' the critical time window in which CSE changes occur. A key difference with previous work however, is that given the CFS paradigm we used, it was crucial to use a fading in of the stimulus to full contrast to achieve stable suppression, whereas no such restriction exists in experiments that focus purely on conscious perception of emotional bodies. This makes it difficult to predict when effects of emotion on M1 would occur. So far, studies on unconscious emotion processing have employed methods such as fMRI and physiological recordings. Albeit interesting with regards to mapping how our brain and body respond to unconscious emotion processing, outcome measures like the BOLD signal, pupil dilation, heart rate or skin conductance are sluggish and lack the power to give any information on when exactly our brain prepares for action in response to unconsciously perceived emotions. One study that did focus on timing of brain responses during unconscious emotion processing, used a combination of backward masking of fearful and neutral faces with EEG (Williams et al., 2004). They found evidence for distinct processing of fearful versus neutral faces within the first $400 \mathrm{~ms}$ of processing. More precisely, unconsciously perceived fear faces elicited a greater N2 component at $200 \mathrm{~ms}$ post stimulus onset, as well as a faster P1 component within 100ms post stimulus onset. No effects for unconsciously perceived faces were found in later components, in contrast to the conscious fearful faces that still modulated the N4 component peaking around $400 \mathrm{~ms}$, suggesting that unconscious emotion stimuli might not be processed beyond an early alerting function.

The only effect of unconscious perception on CSE that we found in the current study was at the early time point $(200 \mathrm{~ms})$, when comparing neutral bodies to control stimuli in the first experiment. In experiment 2 we did not find any evidence for an influence of the unconsciously presented stimuli on CSE at either 200 or $400 \mathrm{~ms}$. As there are thus far no other studies looking into when unconsciously perceived emotion stimuli influence the motor system, this lack of findings might result from probing M1 at the wrong time points.

\section{Differences in fearful and angry body postures as signals of social threat}

Despite both signalling threat, body postures conveying fear or anger might not necessarily evoke the same response when consciously perceived. When perceiving another person in fear, this could reflect fear for something in the environment, or fear for the observer, whereas anger directed at the observer provides an unambiguous threat signal. In accordance with this, neuroimaging studies have shown that perception of fear or anger signals can have distinct neural signatures. Pichon et al. (2009) directly tested the differences in responses to fear versus angry dynamic bodies using fMRI. Whereas both angry and fearful dynamic bodies resulted in similar activations in areas like the amygdala, as well as temporal and prefrontal areas, angry bodies additionally activated anterior temporal regions, premotor cortex, ventro-medial prefrontal cortex and orbitofrontal cortex. These findings concur with the idea that compared to fear, anger signals more directly require behavioural adjustments from the observer. These differences between fear and anger also seem to hold when processed unconsciously, as b-CFS results show that whereas angry bodies break faster from suppression than neutral bodies, fear bodies show the opposite pattern (Zhan et al., 2015).

In experiment 1 we compared CSE changes to fearful bodies with neutral bodies and lamps, and saw in none of our conditions any significant differences between fear and the other 
stimulus categories. In experiment 2 however, we found a significant difference between angry bodies and lamps at $200 \mathrm{~ms}$ in the seen condition. This is in line with previous findings showing CSE modulations in response to angry, but not neutral or fearful, bodies at $300 \mathrm{~ms}$ post stimulus onset (Hortensius et al., 2016). However, we did not test fear and angry bodies in the same experiment, which makes it difficult to conclude whether the observed effects in the angry conditions would also significantly differ from the fearful ones.

\section{CSE as a marker of action preparation in the face of social threat}

MEPs provide a read out of the current state of cortico-spinal excitability at the time that the TMS pulse is administered (Rothwell, 1997), and thus provide a reflection of the functional state of the motor system (Bestmann \& Krakauer, 2015). One issue of using MEPs as the outcome measure of the state of M1, however, is that they provide a compound signal, reflecting not only direct activation (D-waves) of pyramidal tract neurons, but also indirect activation (I-waves) reflecting input onto cortico-spinal pyramidal tract neurons (Rothwell et al., 1999). One way of circumventing this problem is by means of paired pulse stimulation protocols, which allow for the measurement of intracortical facilitation (ICF) and short intracortical inhibition (SICI) (Kujirai et al., 1993; Ziemann, Rothwell, \& Ridding, 1996). In these protocols, first a low-intensity conditioning pulse is administered, followed by a supra-threshold test pulse, and when these two pulses have certain time intervals (1-5ms for SICI and 7-20ms for ICF) this results in either facilitation or inhibition of the following MEP. ICF and SICI are thought to occur at the cortical level, and are thus not 'contaminated' by subcortical or spinal signals, which makes them a more clean read out for the current state of M1.

So despite the fact that CSE as measured with MEPs has been shown to provide a marker for action preparation (Mars, Bestmann, Rothwell, \& Haggard, 2007; van Elswijk, Kleine, Overeem, \& Stegeman, 2007), and MEP modulation in response to social threat has been demonstrated (Borgomaneri et al., 2014; Borgomaneri, Vitale, \& Avenanti, 2015), it is interesting to consider whether ICF and SICI could give a more comprehensive insight into how the motor cortex responds to threat. To date, three published studies have included ICF and SICI as a measure of responding to threatening bodies. Findings showed a reduction of the magnitude of ICF in response to fearful compared to neutral and happy bodies at $100-125 \mathrm{~ms}$ (Borgomaneri, Vitale, Gazzola, et al., 2015), which was later replicated and shown to be larger for participants with a greater self-reported BIS score (Borgomaneri et al., 2017), but this ICF effect was absent at 70-90ms (Borgomaneri, Vitale, \& Avenanti, 2015). No effects on SICI have been found so far. Interestingly, none of these studies included angry bodies as stimuli, which makes it worthwhile to consider how anger threat signals might affect intracortical facilitation and inhibition.

\section{MEPs as a reliable measure of CSE; issues of variability}

Despite being widely used in both research and clinical settings, MEPs suffer from large intraand inter subject variability (Kiers, Cros, Chiappa, \& Fang, 1993). Many factors have been linked as the origin of this variability, like pre-activation of the targeted muscle (Darling, Wolf, \& Butler, 2006), site of the recording electrode (Dunnewold et al., 1998), intensity of the stimulation (Van der Kamp, Zwinderman, Ferrari, \& van Dijk, 1996), state of action preparation (Klein-Flugge, Nobbs, Pitcher, \& Bestmann, 2013), and even gender and age (Pitcher, Ogston, $\&$ Miles, 2003). More recently, spontaneous intrinsic fluctuations of neural oscillatory activity 
have also been implicated in being a source of MEP variability (see e.g. Guerra et al., 2016; Keil et al., 2014; Schulz, Übelacker, Keil, Müller, \& Weisz, 2014).

In the current study we tried to control for the influence of motor preparation in the contralateral hemisphere (responses were made using the left hand) by requiring a motor response in each trial, after a jittered amount of time, and randomizing the response buttons. Muscle preactivation was accounted for by removing all trials that had a pre pulse EMG signal that exceeded a certain threshold. Nonetheless, the observed variability in the first experiment remained high. Therefore, in the second experiment we tried to reduce MEP variability by using navigated TMS, as this has been shown to increase stability of coil positioning (Cincotta et al., 2010). Nonetheless, when calculating the standard deviation for each participant based on all trials (without preprocessing), and then comparing the overall standard deviation between experiment 1 and 2 using the Mann-Whitney test (to account for different sample sizes), we did not see a significant reduction in variability between the two experiments (average SD experiment $1=0.97$, average SD experiment $2=0.96, U=306, p=.863$ ). This is in line with a previous study that systematically compared MEP variability with and without neuronavigation, and did not find significant differences in the coefficient of variance between these two conditions (Jung et al., 2010). So despite the undeniable value that MEPs have in measuring excitability of M1, large variability in data still poses an issue, and possibly can contribute to unclear or even null findings.

\section{Future directions}

Regardless of the findings for influences of threatening postures on CSE in the current study, there are several directions to explore in future studies. First of all, it will be interesting to see whether assessing CSE at different, and specifically earlier, time points will reflect significant modulations of the state of the motor system. EEG findings on masked fearful faces reported results within the first $100 \mathrm{~ms}$ post stimulus onset, and no effects were found in components occurring later than $200 \mathrm{~ms}$ onset (Williams et al., 2004). Similarly, for consciously perceived emotional body postures, effects on CSE can occur as early as $70-90 \mathrm{~ms}$ post stimulus onset (Borgomaneri, Vitale, \& Avenanti, 2015). This makes a window of $100-200 \mathrm{~ms}$ post stimulus onset especially interesting to explore. In addition to this, it will be valuable to assess both fearful and angry postures within the same experimental design, to see if previously suggested differential effects when these signals of threat are consciously perceived also extend to unconscious processing. Lastly, to specifically explore effects of intracortical inhibition and facilitation in the motor system, future studies should compare outcomes of not only MEPs per se, but also include ICF and SICI as measures of interest. 


\section{References}

Almeida, J., Pajtas, P. E., Mahon, B. Z., Nakayama, K., \& Caramazza, A. (2013). Affect of the unconscious: Visually suppressed angry faces modulate our decisions. Cognitive, Affective, \& Behavioral Neuroscience, 13(1), 94-101. https://doi.org/10.3758/s13415-012-0133-7

Bestmann, S., \& Krakauer, J. W. (2015). The uses and interpretations of the motor - evoked potential for understanding behaviour, 679-689. https://doi.org/10.1007/s00221-014-4183-7

Borgomaneri, S., Gazzola, V., \& Avenanti, A. (2012). Motor mapping of implied actions during perception of emotional body language. Brain Stimulation, 5(2), 70-6. https://doi.org/10.1016/j.brs.2012.03.011

Borgomaneri, S., Gazzola, V., \& Avenanti, A. (2014). Transcranial magnetic stimulation reveals two functionally distinct stages of motor cortex involvement during perception of emotional body language. Brain Structure \& Function. https://doi.org/10.1007/s00429-014-0825-6

Borgomaneri, S., Vitale, F., \& Avenanti, A. (2015). Early changes in corticospinal excitability when seeing fearful body expressions. Scientific Reports, 5, 14122. https://doi.org/10.1038/srep14122

Borgomaneri, S., Vitale, F., \& Avenanti, A. (2017). Behavioral inhibition system sensitivity enhances motor cortex suppression when watching fearful body expressions. Brain Structure and Function, O(0), 0. https://doi.org/10.1007/s00429-017-1403-5

Borgomaneri, S., Vitale, F., Gazzola, V., \& Avenanti, A. (2015). Seeing fearful body language rapidly freezes the observer's motor cortex. Cortex, 65, 232-245. https://doi.org/10.1016/j.cortex.2015.01.014

Bradley, M. M., Miccoli, L., Escrig, M. A., \& Lang, P. J. (2008). The pupil as a measure of emotional arousal and autonomic activation. Psychophysiology, 45(4), 602-607. https://doi.org/10.1111/j.1469-8986.2008.00654.x

Bucchioni, G., Cavallo, A., Ippolito, D., Marton, G., \& Castiello, U. (2013). Corticospinal excitability during the observation of social behavior. Brain and Cognition, 81(2), 176-82. https://doi.org/10.1016/j.bandc.2012.11.001

Carlson, J. M., \& Reinke, K. S. (2008). Masked fearful faces modulate the orienting of covert spatial attention. Emotion, 8(4), 522.

Cincotta, M., Giovannelli, F., Borgheresi, A., Balestrieri, F., Toscani, L., Zaccara, G., ... Rossi, S. (2010). Optically tracked neuronavigation increases the stability of hand-held focal coil positioning: Evidence from "transcranial" magnetic stimulation-induced electrical field measurements. Brain Stimulation, 3(2), 119-123. https://doi.org/10.1016/j.brs.2010.01.001

Coelho, C. M., Lipp, O. V., Marinovic, W., Wallis, G., \& Riek, S. (2010). Increased corticospinal excitability induced by unpleasant visual stimuli. Neuroscience Letters, 481(3), 135-138. https://doi.org/10.1016/j.neulet.2010.03.027

Coombes, S. A., Tandonnet, C., Fujiyama, H., Janelle, C. M., Cauraugh, J. H., \& Summers, J. J. (2009). Emotion and motor preparation: A transcranial magnetic stimulation study of corticospinal motor tract excitability. Cognitive, Affective \& Behavioral Neuroscience, 9(4), 380-388. https://doi.org/10.3758/CABN.9.4.380

Darling, W. G., Wolf, S. L., \& Butler, A. J. (2006). Variability of motor potentials evoked by transcranial 
magnetic stimulation depends on muscle activation. Experimental Brain Research, 174(2), 376385. https://doi.org/10.1007/s00221-006-0468-9

de Gelder, B., Snyder, J., Greve, D., Gerard, G., \& Hadjikhani, N. (2004). Fear fosters flight: a mechanism for fear contagion when perceiving emotion expressed by a whole body. Proceedings of the National Academy of Sciences of the United States of America, 101(47), 16701-6. https://doi.org/10.1073/pnas.0407042101

de Gelder, B., Vroomen, J., Pourtois, G., \& Weiskrantz, L. (1999). Non-conscious recognition of affect in the absence of striate cortex. NeuroReport, 10(18), 3759-3763. https://doi.org/10.1097/00001756-199912160-00007

Dimberg, U., Thunberg, M., \& Elmehed, K. (2000). Unconscious Facial Reactions to Emotional Facial Expressions. Psychological Science, 11(1), 86-89. https://doi.org/10.1111/1467-9280.00221

Dunnewold, R. J. W., Van Der Kamp, W., Van Den Brink, A. M., Stijl, M., Reegt, \& Van Dijk, J. G. (1998). Influence of electrode site and size on variability of magnetic evoked potentials. Muscle and Nerve, 21(12), 1779-1782. https://doi.org/10.1002/(SICI)1097- ４598(199812)21:12

Eder, A. B., \& Rothermund, K. (2013). Emotional action: An ideomotor model. In C. Mohiyeddini, M. W. Eysenck, \& S. Bauer (Eds.), Handbook of psychology of emotions (1st ed., pp. 11-38). Hauppauge, NY: Nova Science.

Esteves, F., Dimberg, U., \& Öhman, A. (1994). Automatically elicited fear: Conditioned skin conductance responses to masked facial expressions. Cognition \& Emotion, 8(5), 393-413.

Fadiga, L., Craighero, L., \& Olivier, E. (2005). Human motor cortex excitability during the perception of others' action. Current Opinion in Neurobiology, 15(2), 213-218. https://doi.org/10.1016/j.conb.2005.03.013

Frijda, N. H. (2010). Impulsive action and motivation. Biological Psychology, 84(3), 570-579. https://doi.org/10.1016/j.biopsycho.2010.01.005

Goldberg, H., Preminger, S., \& Malach, R. (2014). The emotion-action link? Naturalistic emotional stimuli preferentially activate the human dorsal visual stream. NeuroImage, 84, 254-64. https://doi.org/10.1016/j.neuroimage.2013.08.032

Guerra, A., Pogosyan, A., Nowak, M., Tan, H., Ferreri, F., Di Lazzaro, V., \& Brown, P. (2016). Phase Dependency of the Human Primary Motor Cortex and Cholinergic Inhibition Cancelation During Beta tACS. Cerebral Cortex, 1-15. https://doi.org/10.1093/cercor/bhw245

Hajcak, G., Molnar, C., George, M. S., Bolger, K., Koola, J., \& Nahas, Z. (2007). Emotion facilitates action: a transcranial magnetic stimulation study of motor cortex excitability during picture viewing. Psychophysiology, 44(1), 91-7. https://doi.org/10.1111/j.1469-8986.2006.00487.x

Hamm, A. O., Weike, A. I., Schupp, H. T., Treig, T., Dressel, A., \& Kessler, C. (2003). Affective blindsight: Intact fear conditioning to a visual cue in a cortically blind patient. Brain, 126(2), 267-275. https://doi.org/10.1093/brain/awg037

Hortensius, R., de Gelder, B., \& Schutter, D. J. L. G. (2016). When anger dominates the mind: Increased motor corticospinal excitability in the face of threat. Psychophysiology, 53(9), 1307-1316. https://doi.org/10.1111/psyp.12685

Huis In 't Veld, E. M. J., Van Boxtel, G. J. M., \& de Gelder, B. (2014). The Body Action Coding System I: muscle activations during the perception and expression of emotion. Social Neuroscience, 9(3), 
249-64. https://doi.org/10.1080/17470919.2014.890668

Jolij, J., \& Lamme, V. A. F. (2005). Repression of unconscious information by conscious processing: Evidence from affective blindsight induced by transcranial magnetic stimulation. Proceedings of the National Academy of Sciences, 102(30), 10747-10751. https://doi.org/10.1073/pnas.0500834102

Jung, N. H., Delvendahl, I., Kuhnke, N. G., Hauschke, D., Stolle, S., \& Mall, V. (2010). Navigated transcranial magnetic stimulation does not decrease the variability of motor-evoked potentials. Brain Stimulation, 3(2), 87-94. https://doi.org/10.1016/j.brs.2009.10.003

Keil, J., Timm, J., Sanmiguel, I., Schulz, H., Obleser, J., \& Schönwiesner, M. (2014). Cortical brain states and corticospinal synchronization influence TMS-evoked motor potentials. Journal of Neurophysiology, 111(3), 513-9. https://doi.org/10.1152/jn.00387.2013

Kiers, L., Cros, D., Chiappa, K. H., \& Fang, J. (1993). Variability of motor potentials evoked by transcranial magnetic stimulation. Electroencephalography and Clinical Neurophysiology/ Evoked Potentials, 89(6), 415-423. https://doi.org/10.1016/0168-5597(93)90115-6

Klein-Flugge, M. C., Nobbs, D., Pitcher, J. B., \& Bestmann, S. (2013). Variability of Human Corticospinal Excitability Tracks the State of Action Preparation. Journal of Neuroscience, 33(13), 5564-5572. https://doi.org/10.1523/JNEUROSCI.2448-12.2013

Kujirai, T., Caramia, M. D., Rothwell, J. C., Day, B. L., Thompson, P. D., Ferberts, A., ... Marsen, C. D. (1993). Corticocortical inhibition in human motor cortex. Journal of Physiology, 471(1), 501-519.

Mars, R. B., Bestmann, S., Rothwell, J. C., \& Haggard, P. (2007). Effects of motor preparation and spatial attention on corticospinal excitability in a delayed-response paradigm. Experimental Brain Research, 182(1), 125-129. https://doi.org/10.1007/s00221-007-1055-4

Mattiassi, A. D. ., Mele, S., Ticini, L. F., \& Urgesi, C. (2014). Conscious and Unconscious Representations of Observed Actions in the Human Motor System. Journal of Cognitive Neuroscience, 26(9), 20282041. https://doi.org/10.1162/jocn

Meeren, H. K. M., Hadjikhani, N., Ahlfors, S. P., Hämäläinen, M. S., \& de Gelder, B. (2016). Early Preferential Responses to Fear Stimuli in Human Right Dorsal Visual Stream - A MEG Study. Scientific Reports, 6(April), 24831. https://doi.org/10.1038/srep24831

Pichon, S., de Gelder, B., \& Grèzes, J. (2009). Two different faces of threat. Comparing the neural systems for recognizing fear and anger in dynamic body expressions. NeuroImage, 47(4), 1873-1883. https://doi.org/10.1016/j.neuroimage.2009.03.084

Pitcher, J. B., Ogston, K. M., \& Miles, T. S. (2003). Age and sex differences in human motor cortex inputoutput characteristics. The Journal of Physiology, 546(2), 605-613. https://doi.org/10.1113/jphysiol.2002.029454

Roelofs, K., Hagenaars, M. A., \& Stins, J. (2010). Facing freeze: social threat induces bodily freeze in humans. Psychological Science, 21(11), 1575-1581. https://doi.org/10.1177/0956797610384746

Rossi, S., Hallett, M., Rossini, P. M., \& Pascual-Leone, A. (2012). Safety, ethical considerations, and application guidelines for the use of transcranial magnetic stimulation in clinical practice and research. Clinical Neurophysiology, $\quad 120(12), \quad 323-330$. https://doi.org/10.1016/j.clinph.2009.08.016.Rossi

Rothwell, J. C. (1997). Techniques and mechanisms of action of transcranial stimulation of the human 88 
motor cortex. Journal of Neuroscience Methods, 74(2), 113-122. https://doi.org/10.1016/S0165$0270(97) 02242-5$

Rothwell, J. C., Hallett, M., Berardelli, A., Eisen, A., Rossini, P., \& Paulus, W. (1999). Magnetic stimulation: motor evoked potentials. The International Federation of Clinical Neurophysiology. Electroencephalography and Clinical Neurophysiology. Supplement, 52, 97-103.

Ruiz-Padial, E., Mata, J. L., Rodríguez, S., Fernández, M. C., \& Vila, J. (2005). Non-conscious modulation of cardiac defense by masked phobic pictures. International Journal of Psychophysiology, 56(3), 271281. https://doi.org/10.1016/j.ijpsycho.2004.12.010

Ruiz-Padial, E., Vila, J., \& Thayer, J. F. (2011). The effect of conscious and non-conscious presentation of biologically relevant emotion pictures on emotion modulated startle and phasic heart rate. International Journal of Psychophysiology, 79(3), 341-346. https://doi.org/10.1016/j.ijpsycho.2010.12.001

Schulz, H., Übelacker, T., Keil, J., Müller, N., \& Weisz, N. (2014). Now i am ready - Now i am not: The influence of pre-TMS oscillations and corticomuscular coherence on motor-evoked potentials. Cerebral Cortex, 24(7), 1708-1719. https://doi.org/10.1093/cercor/bht024

Schurger, A. (2009). A very inexpensive MRI-compatible method for dichoptic visual stimulation. Journal of Neuroscience Methods, 177(1), 199-202. https://doi.org/10.1016/j.jneumeth.2008.09.028

Schutter, D. J. L. G., Hofman, D., \& Van Honk, J. (2008). Fearful faces selectively increase corticospinal motor tract excitability: A transcranial magnetic stimulation study. Psychophysiology, 45(3), 345348. https://doi.org/10.1111/j.1469-8986.2007.00635.x

Stein, T., Sterzer, P., \& Peelen, M. V. (2012). Privileged detection of conspecifics: Evidence from inversion effects during continuous flash suppression. Cognition, 125(1), 64-79. https://doi.org/10.1016/j.cognition.2012.06.005

Tamietto, M., Castelli, L., Vighetti, S., Perozzo, P., Geminiani, G., Weiskrantz, L., \& de Gelder, B. (2009). Unseen facial and bodily expressions trigger fast emotional reactions. Proceedings of the National Academy of Sciences of the United States of America, 106(42), 17661-17666. https://doi.org/10.1073/pnas.0908994106

Tamietto, M., Cauda, F., Celeghin, A., Diano, M., Costa, T., Cossa, F. M., ... de Gelder, B. (2015). Once you feel it, you see it: Insula and sensory-motor contribution to visual awareness for fearful bodies in parietal neglect. Cortex, 62, 56-72. https://doi.org/10.1016/j.cortex.2014.10.009

Tamietto, M., \& de Gelder, B. (2008a). Affective blindsight in the intact brain: Neural interhemispheric summation for unseen fearful expressions. Neuropsychologia, 46(3), 820-828. https://doi.org/10.1016/j.neuropsychologia.2007.11.002

Tamietto, M., \& de Gelder, B. (2008b). Emotional contagion for unseen bodily expressions: evidence from facial emg. 2008 8th IEEE International Conference on Automatic Face and Gesture Recognition, FG 2008, 1-5. https://doi.org/10.1109/AFGR.2008.4813317

Tamietto, M., \& de Gelder, B. (2010). Neural bases of the non-conscious perception of emotional signals. Nature Reviews. Neuroscience, 11(10), 697-709. https://doi.org/10.1038/nrn2889

Théoret, H., Halligan, E., Kobayashi, M., Merabet, L., \& Pascual-Leone, A. (2004). Unconscious modulation of motor cortex excitability revealed with transcranial magnetic stimulation. Experimental Brain Research, 155(2), 261-4. https://doi.org/10.1007/s00221-003-1820-y 
Théoret, H., Kobayashi, M., Merabet, L., Wagner, T., Tormos, J. M., \& Pascual-Leone, A. (2004). Modulation of right motor cortex excitability without awareness following presentation of masked self-images. Brain Research. Cognitive Brain Research, 20(1), 54-7. https://doi.org/10.1016/j.cogbrainres.2004.01.003

Tsuchiya, N., \& Koch, C. (2005). Continuous flash suppression reduces negative afterimages. Nature Neuroscience, 8(8), 1096-1101. https://doi.org/10.1038/nn1500

Van den Stock, J., Tamietto, M., Sorger, B., Pichon, S., Grézes, J., \& de Gelder, B. (2011). Corticosubcortical visual, somatosensory, and motor activations for perceiving dynamic whole-body emotional expressions with and without striate cortex (V1). Proceedings of the National Academy of Sciences of the United States of America, 108(39), 16188-93. https://doi.org/10.1073/pnas.1107214108

Van der Kamp, W., Zwinderman, A. H., Ferrari, M. D., \& van Dijk, J. G. (1996). Cortical excitability and response variability of transcranial magnetic stimulation. Journal of Clinical Neurophysiology, 13(2), $164-171$.

van Elswijk, G., Kleine, B. U., Overeem, S., \& Stegeman, D. F. (2007). Expectancy induces dynamic modulation of corticospinal excitability. Journal of Cognitive Neuroscience, 19, 121-131. https://doi.org/10.1162/jocn.2007.19.1.121

Van Honk, J., Tuiten, A., Van Den Hout, M., Koppeschaar, H., Thijssen, J., De Haan, E., \& Verbaten, R. (1998). Baseline salivary cortisol levels and preconscious selective attention for threat: A pilot study. Psychoneuroendocrinology, 23(7), 741-747. https://doi.org/10.1016/S03064530(98)00047-X

van Loon, A. M., van den Wildenberg, W. P. M., van Stegeren, A. H., Ridderinkhof, K. R., \& Hajcak, G. (2010). Emotional stimuli modulate readiness for action: A transcranial magnetic stimulation study. Cognitive, Affective, \& Behavioral Neuroscience, 10(2), 174-181. https://doi.org/10.3758/CABN.10.2.174

Williams, L. M., Liddell, B. J., Rathjen, J., Brown, K. J., Gray, J., Phillips, M., ... Gordon, E. (2004). Mapping the Time Course of Nonconscious and Conscious Perception of Fear: An Integration of Central and Peripheral Measures. Human Brain Mapping, 21(2), 64-74. https://doi.org/10.1002/hbm.10154

Zhan, M., Hortensius, R., \& de Gelder, B. (2015). The body as a tool for anger awareness-differential effects of angry facial and bodily expressions on suppression from awareness. PLoS ONE, 10(10), 1-13. https://doi.org/10.1371/journal.pone.0139768

Ziemann, U., Rothwell, J. C., \& Ridding, M. C. (1996). Interaction between intracortical inhibition and facilitation in human motor cortex. The Journal of Physiology, 496 ( Pt 3, 873-81. https://doi.org/10.1113/jphysiol.1996.sp021734 


\section{Chapter Phase of beta-frequency tACS over primary motor cortex modulates corticospinal excitability}

Based on:

Engelen, T.*, Schilberg, L.*, ten Oever, S., Schuhmann, T., de Gelder, B., de Graaf, T. A., \& Sack, A. T. (2018).

Phase of beta-frequency tACS over primary motor cortex modulates corti- cospinal excitability. Cortex, 103, 142-152. 



\begin{abstract}
The assessment of corticospinal excitability by means of TMS-induced MEPs is an established diagnostic tool in neurophysiology and a widely used procedure in fundamental brain research. However, concern about low reliability of these measures has grown recently. One possible cause of high variability of MEPs under identical acquisition conditions could be the influence of oscillatory neuronal activity on corticospinal excitability. Based on research showing that tACS can entrain neuronal oscillations we here test whether alpha or beta frequency tACS can influence corticospinal excitability in a phase-dependent manner. We applied tACS at individually calibrated alpha- and beta-band oscillation frequencies, or we applied sham tACS. Simultaneous single TMS pulses time locked to eight equidistant phases of the ongoing tACS signal evoked MEPs. To evaluate offline effects of stimulation frequency, MEP amplitudes were measured before and after tACS. To evaluate whether tACS influences MEP amplitude, we fitted one-cycle sinusoids to the average MEPs elicited at the different phase conditions of each tACS frequency. We found no frequency-specific offline effects of tACS. However, betafrequency tACS modulation of MEPs was phase-dependent. Post hoc analyses suggested that this effect was specific to participants with low $(<19 \mathrm{~Hz})$ intrinsic beta frequency. In conclusion, by showing that beta tACS influences MEP amplitude in a phase-dependent manner, our results support a potential role attributed to neuronal oscillations in regulating corticospinal excitability. Moreover, our findings may be useful for the development of TMS protocols that improve the reliability of MEPs as a meaningful tool for research applications or for clinical monitoring and diagnosis.
\end{abstract}

\title{
Introduction
}

Non-invasive stimulation of the human brain is widely applied in research and clinical settings. One commonly used application is the assessment of corticospinal excitability. For that, transcranial magnetic stimulation (TMS) over the motor cortex is combined with electromyography (EMG) from peripheral muscles (e.g. hand muscles) to induce and measure motor evoked potentials (MEPs) (Rothwell et al., 1999). The magnitude of such MEPs reflects corticospinal excitability and their evaluation can be of great benefit in both research and clinical applications (Rossini \& Rossi, 1998).

MEPs are usually evaluated at the beginning of most TMS experiments or before TMS treatment to determine stimulation intensity. In addition, measuring MEPs allows for the investigation of intra- and interhemispheric connections and interactions (Bestmann \& Krakauer, 2014), and can provide information about neuroplasticity mechanisms (Pascual-Leone, Tormos, \& Keenan, 1998; Moliadze, Fritzsche \& Antal 2014) or specific intervention efficacies (Di Lazzaro et al., 1999). Moreover, MEP assessments can be useful in diagnosis of neurological diseases (Ni \& Chen, 2015), such as Alzheimer's disease (Di Lazzaro et al., 2004; Ferreri et al., 2002), Parkinson disease (Chen, Kumar, Garg \& Lang, 2001; Lefaucheur, 2005) or multiple sclerosis (Fuhr, Borggrefe-Chappuis, Schindler \& Kappos, 2001; Kallmann, Facklemann, Toyka, RIekmann \& Reiners., 2006; Rico et al., 2009).

Although widely implemented in research and clinic, such measures of corticospinal excitability display substantial and largely unexplained within- and between-subject variability (Jung et al., 2010; Kiers, Cros, Chiappa, \& Fang, 1993; Rösler, Roth, \& Magistris, 2008). While being a valuable average measurement tool on the group level, this unexplained variability reduces the reliability of corticospinal excitability assessments with TMS, severely hampering its value as a 
diagnostic or scientific marker for a given individual participant or patient. Revealing factors that modulate corticospinal excitability measures would add to our fundamental understanding of the motor system and human cortical excitability mechanisms. Furthermore, reducing variability could have direct experimental and clinical implications, as the reliability of TMS-induced MEP measures of corticospinal excitability would be increased, thereby both allowing for greater applicability of this technique as well as increasing the validity of corticospinal excitability assessments.

Neuronal networks within and across brain systems routinely engage in synchronized rhythmic activity to establish connectivity between neuronal ensembles and allow for the selection or maintenance of information (Buzsáki \& Draguhn, 2004). When looking at sources of variability in corticospinal excitability measures, it seems that such neuronal oscillations may cause, or reflect, fluctuations in cortical excitability (Mäki \& Ilmoniemi, 2010). In humans, electroencephalography (EEG) can be used to measure oscillatory brain activity at the scalp. The overall strength of oscillations in a particular frequency-band is reflected by the power (directly related to amplitude) of the EEG signal, while its phase reflects the momentary state (e.g. peak or trough) of the ongoing rhythm. Different brain systems inherently seem to operate in different frequency bands. Beta frequency $(13-30 \mathrm{~Hz})$ is the main frequency of the sensorimotor system (Niedermeyer, 2005; Feurra et al., 2011; Kilavik, Zaepffel, Brovelli, Mackay \& Riehle, 2013) and alpha frequency is suggested to be involved in cortical inhibition (Pfurtscheller, Stancák, \& Neuper, 1996; Jensen \& Mazaheri, 2010; Mathewson et al., 2011).

In the current study, we measured EMG while simultaneously applying TMS and transcranial alternating current stimulation (tACS) to investigate whether tACS at alpha and beta frequency modulates MEP amplitude. tACS is a periodic electrical stimulation of the human cortex, effectively enabling modulation over oscillations (Antal \& Paulus, 2013). Thereby, specific oscillatory frequencies can be entrained in the targeted brain areas (Herrmann, Rach, Neuling \& Strüber, 2013). When TMS is applied simultaneously to the same target region it is possible to control the timing of the TMS pulse and lock it to particular phases of the entraining tACS signal (ten Oever et al., 2016; Raco, Bauer, Tharsan, \& Gharabaghi, 2016). Enhancement of the amplitude of intrinsic neuronal oscillations occurs both online during tACS (Fröhlich \& McCormick, 2010) and offline following tACS (Zaehle, Rach \& Herrmann, 2010; Neuling, Rach \& Herrmann, 2013). The effects of tACS entrainment over M1 at both alpha and beta frequencies are specific to brain areas immediately underneath and in close proximity to the stimulation electrodes, including pre- and post-central gyri (Witkowski et al., 2016).

We recently developed and validated a setup that allows administration of TMS pulses (or indeed any (multi-) sensory stimulation) time-locked to tACS frequency and phase with submillisecond precision (ten Oever et al., 2016). This setup enables the investigation of how the ongoing tACS signal influences MEP amplitude. Here, TMS pulses were delivered at eight equidistant phases of a sinusoidal tACS signal applied to the motor cortex (Figure 1A). We administered tACS at either individual peak alpha frequency $(8-13 \mathrm{~Hz})$, individual peak beta frequency $(16-25 \mathrm{~Hz}$ ), or tACS was simulated (sham tACS). We performed several analyses that directly evaluated whether data revealed a one-cycle oscillatory pattern of MEP amplitudes across the eight TMS-tACS phase conditions. We hypothesized that a causal role of tACS frequency and phase would lead to mean differences in MEP amplitudes when comparing different frequencies and phase conditions. More stringently, we expected to show tACS frequencydependence during and after the stimulation by comparing MEP amplitude elicited by TMSonly trials (without tACS) with MEP amplitudes during and after combined tACS-TMS. 
Finally, we expected tACS signal phase-locking of TMS to results in an oscillatory pattern of MEP amplitudes across the eight TMS-tACS phase conditions, thereby showing tACS phasedependence of corticospinal excitability.

\section{Methods}

\section{Participants}

Fifteen healthy participants completed all three sessions of the experiment (10 females, mean age $=24.4( \pm 3.7 \mathrm{SD})$ ). All participants were right handed and had normal or corrected to normal vision. Participants were screened for TMS safety based on published safety guidelines (Rossi, Hallett, Rossini, \& Pascual-Leone, 2012). All participants gave written informed consent before taking part in the experiment. The study was performed in accordance to the Declaration of Helsinki and it was approved by the local ethical committee.

\section{Individual EEG localizers}

To establish optimal individualized stimulation frequencies for tACS, each participant completed a short EEG localizer task at the beginning of the alpha and beta session. EEG data was recorded using BrainAmp MR Plus EEG amplifiers and BrainVision recorder (Brain products, Gilching, Germany) with a sampling rate of $2500 \mathrm{~Hz}$, using a hardware bandpass filter of $0.1-1000 \mathrm{~Hz}$, a software low pass filter of $250 \mathrm{~Hz}$, and a notch filter at $50 \mathrm{~Hz}$. Three EEG electrodes were placed according to the 10-20 system over C3 (motor cortex), Cz (ground) and right mastoid (reference) using Ten20 paste (Weaver, Colorado). Recording was not started until an impedance of $<20 \mathrm{kOhm}$ was established. During the individual alpha localizer, participants were instructed to sit in a relaxed position with their eyes closed for five minutes while EEG was recorded. For the individual beta localizer, participants were instructed to voluntarily press a button with their right index finger (approximately every six seconds) for ten minutes while EEG was recorded. This led to a total of ca. 70 button presses per participant (similar to Romei et al., 2016).

\section{TMS parameters}

In order to assure stimulation location accuracy throughout each session the BrainVoyager neuronavigation system (Brain Innovation B.V., Maastricht, The Netherlands) was used. Single TMS pulses were applied over M1 in the left hemisphere. Magnetic stimulation was applied before, during, and after tACS stimulation at $120 \%$ of individual resting motor threshold (mean stimulation intensity $=50 \%$ ( $\pm 6.1 \mathrm{SD}$ ) of maximum stimulator output) using a MC-B70 figureof-eight coil and a Magpro X100 stimulator (Magventure A/S, Farum, Denmark). Individual motor thresholds were defined as the lowest stimulation intensity needed to evoke an MEP $(>$ $0.05 \mathrm{mV})$ in five out of ten pulses. The inter pulse interval was jittered around $6.75( \pm 0.75)$ seconds. The coil was placed tangentially to the scalp with the handle pointing backwards at a $45^{\circ}$ angle to the midline. Placement location was determined based on an optimal position for induction of maximal MEPs in the contralateral target muscle. All delivered pulses were biphasic with the current flowing in an antero-posterior and then postero-anterior (AP - PA) direction in the brain. Each TMS pulse triggered electromyography recordings of the right first dorsal interosseous (FDI) muscle for $100 \mathrm{~ms}$ pre- until $150 \mathrm{~ms}$ post pulse. To measure EMG of the FDI muscle, disposable adhesive silver-chloride surface electrodes (Plaquette ${ }^{\mathrm{TM}}$, Technomed Europe) were placed in a belly-tendon montage with a ground electrode on the wrist. 
EMG signals were recorded using a Powerlab 4/35 with a Bio Amp system (ADInstruments, Sydney, Australia). The signal was amplified, sampled at $4 \mathrm{k} / \mathrm{s}$, digitized and stored on a computer for offline analysis.

\section{tACS parameters}

tACS stimulation was applied using a DC-stimulator plus (NeuroConn, Ilmenau, Germany). After removal of the EEG electrodes, two $3 \times 3 \mathrm{~cm}$ standard tACS electrodes were fixated using conductive EEG gel (Ten20 conductive Neurodiagnostic electrode paste, WEAVER and company, Aurora CO, USA). One tACS-electrode was applied over the predefined individual motor hotspot in the left hemisphere, and the other tACS-electrode was applied on location $\mathrm{Pz}$ based on the international 10-20 system. The intensity of stimulation was fixed for every participant at $1.5 \mathrm{~mA}$ peak-to-peak, with a ramping up and down phase of 10 seconds. External control of tACS can involve very small DC offsets (here, using the NeuroConn 'Remote' feature) of likely negligible influence. Nevertheless, we here reduced, but not completely removed, these offsets by adapting source files driving both tACS and TMS stimulation (see ten Oever et al., 2016). During each session the stimulation frequency was set to either individual alpha or beta frequency for each participant. The total time of stimulation was 36.5 minutes. Positioning of the tACS electrodes was identical in the sham session to ensure similar distances between the TMS coil and the scalp, only the wires were not plugged into the stimulator (not visible for participants). There was no ramping up or down during the Sham-Visit.

\section{Procedure}

Participants came in for three sessions (alpha, beta, sham tACS), with the order of conditions being counterbalanced across participants. Alpha and beta tACS sessions started with EEG localizers. EMG and tACS electrodes were placed on the hand and head of the participants. Electrode placement was optimized to yield resting EMG signal below $0.05 \mathrm{mV}$ (peak-to-peak). Neuronavigation emitters were fixated on the participants' heads, which were co-registered to a dummy head in BrainVoyager for accurate and stable coil placement throughout each session. The optimal location for stimulation was determined by systematically searching for the hand hotspot with single TMS pulses over left M1. Once the best location for the stimulation of the FDI muscle was found, one tACS-electrode was placed over this hotspot (see tACS parameters). The TMS coil was fixed into position on top of this electrode using a coil holder, while the participant rested their head in a chinrest. In addition, the coil location was registered in the BrainVoyager Neuronavigation system to ensure the stimulation site did not vary during the session. After the coil was fixed into the optimal position, resting motor threshold was assessed. Before the start of tACS entrainment, a baseline of corticospinal excitability was measured by applying 40 pulses at $120 \%$ of resting motor threshold and recording the corresponding MEPs. Then, the tACS stimulation was used to entrain motor cortex oscillations at either individual alpha (mean $=10.2 \mathrm{~Hz})$, beta $($ mean $=18.9 \mathrm{~Hz})$ or sham frequency, while single TMS pulses were applied at eight equidistant phases of one oscillatory cycle of the tACS signal (Figure 1A, see table 1 for all individual tACS stimulation frequencies). tACS stimulation lasted for a total of 36.5 minutes while 40 TMS pulses per phase bin were randomly administered. Phase locking of TMS pulses to the tACS signal was established through in house software (Data Streamer), for more details see ten Oever et al. (2016). After the completion of tACS entrainment, another 40 TMS pulses were applied to assess tACS after-effects on corticospinal excitability. For an overview of the experimental design see Figure 1B. 
A

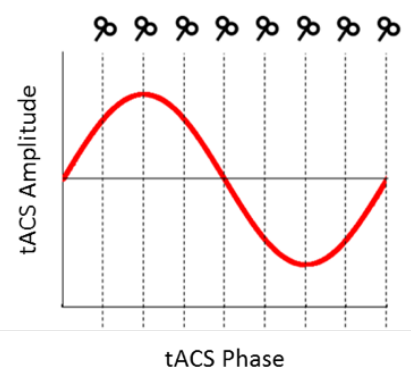

B

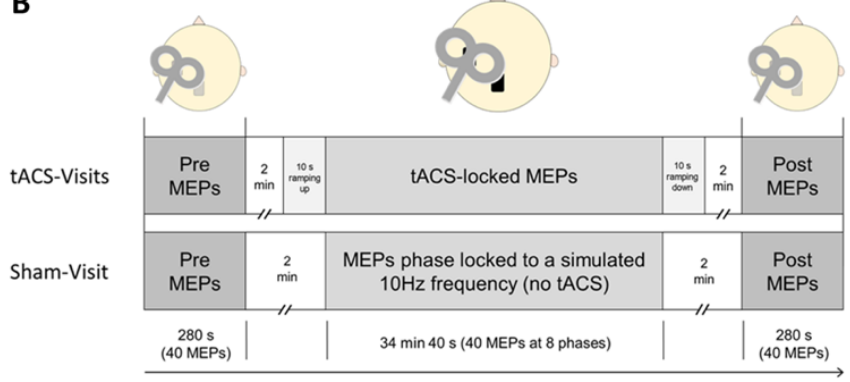

Figure 1. A) TMS pulses were administered at eight equidistantly spaced phases of a one-cycle sinusoidal tACS signal applied over M1. The order of TMS administration was randomized with a total of forty pulses per phase. B) Overview of the experimental design. Measures of 40 spTMS-elicited MEPs were conducted two minutes before and after the application of combined tACS-spTMS. During the Alpha-Visit and Beta-Visit this combined protocol consisted of tACS entrainment at either individually predefined alpha or beta frequency together with spTMS phase locked to this tACS frequency. tACS was ramped up for ten seconds before and ramped down for ten seconds after the combined tACS-TMS protocol. During Sham-Visit tACS electrodes were attached, but not connected to the amplifier, while spTMS was applied phase-locked to a simulated $10 \mathrm{~Hz}$ frequency. The order of the visits was counterbalanced across participants.

\section{Analysis}

\section{Individual EEG localizers}

All EEG data were pre-processed and analysed using the FieldTrip toolbox (Oostenveld, Fries, Maris, \& Schoffelen, 2011). The individual beta frequency was determined by measuring the dominant frequency from the cortex one second after voluntary muscle movement (beta rebound) (Romei et al., 2016; Witkowski et al., 2016 Jurkiewicz, Gaetz, Bostan, \& Cheyne, 2006; Pfurtscheller, Stancák, \& Neuper, 1996). EEG data of the beta localizer were epoched into -2 to +2 seconds time windows around the button press and demeaned. Trials with high variance were removed based on visual inspection. A time-frequency analysis was performed applying wavelets for frequencies ranging from 0.25 to $30 \mathrm{~Hz}$ in steps of $0.25 \mathrm{~Hz}$. The width of the wavelets increased linearly from 2 to 10 cycles. Power values were normalized as a relative change from a baseline period ( -1 to $-0.5 \mathrm{sec}$ to button presses). Finally, the peak beta frequency in a frequency range of $14-25 \mathrm{~Hz}$ averaging over a $0.3-0.8 \mathrm{sec}$ time window was extracted. Some participants repeated the task as no clear visual peak was present in the initial recording of the voluntary button press task.

In the individual alpha EEG localizer, participants kept their eyes closed for five minutes. Resulting EEG data were arbitrarily epoched to $5000 \mathrm{~ms}$ segments, on which a Fourier transform was done to yield a power spectrum per epoch with a frequency resolution of $0.2 \mathrm{~Hz}$. These 
spectra were averaged across epochs. Individual alpha frequency was defined as the frequency in the $7-13 \mathrm{~Hz}$ range of the spectrum with the local maximum power value (alpha 'peak').

\section{MEP pre-processing}

Neurophysiological data were processed offline. Mean MEP peak-to-peak values were calculated in $\mathrm{mV}$ using Labchart 8 (ADInstruments, Sydney, Australia). To rule out any effects of muscle pre-contraction on MEP amplitude, all trials with pre-pulse peak-to-peak values deviating more than 3 SDs from the average of all $100 \mathrm{~ms}$ pre-pulse values were excluded $(<5 \%$ of trials). Additionally, any trials with MEP peak-to-peak values below $0.05 \mathrm{mV}$ were removed. After initial cleaning of the data, outliers deviating more than 3 SDs from the mean MEP values of each condition (10 conditions in total representing each phase bin and pre- and post MEPs) were removed. Average MEPs per phase were normalized for each individual using the average across all phases. To ensure that the EMG signal, and therefore also the MEP results, were not confounded by the concurrent application of tACS, the data was additionally filtered with a high-pass filter at $30 \mathrm{~Hz}$ (falling well above the highest used tACS stimulation frequency of $25 \mathrm{~Hz})$ ) using the FieldTrip Toolbox (one pass, FIR-filter, filter order $=100$ ).

\section{The effect of $\mathrm{ACS}$ at alpha and beta frequency on corticospinal excitability}

We evaluated the effect of tACS at alpha and beta frequencies on corticospinal excitability by comparing MEPs before, during, and after tACS. We performed a repeated measures ANOVA with time (pre/during/post) and tACS (alpha/beta/sham) as within subject factors. Individual MEPs, after pre-processing, were averaged per time and tACS condition. Greenhouse-Geisser correction was used in case of violation of the sphericity assumption as indicated by Mauchley's test.

\section{The effect of $t A C S$ phase on corticospinal excitability}

To assess the effect of tACS phase MEP amplitudes were averaged for each phase bin per participant, and normalized to the individual mean MEP across phase bins. This yielded patterns of MEPs over tACS oscillatory phase bins. If, as hypothesized, tACS phase is relevant for MEP amplitude, then these patterns should depict a single sinusoidal cycle. Thus, the core of our analysis was to fit one-cycle sinusoids on MEP patterns (MEP over the 8 phase bins). We performed different such analyses. In group analyses, we averaged the normalized MEP patterns over participants, per tACS condition, and fit a one-cycle sinusoid on the resulting group pattern. In subject analyses, we fit one-cycle sinusoids on individual MEP patterns, and performed second-level statistical analyses on the measures of fit.

In all cases, one-cycle sinusoids were fit to MEP patterns (per condition alpha/beta/sham) using the Matlab function 'fminsearch' (based on the parameters phase, amplitude and mean). We calculated explained variance (R-squared) measures of best-fitting sinusoids, and multiplied them by the variance of predicted values, to obtain the established 'relevance value' (Fiebelkorn et al., 2011). The relevance value takes into consideration both amplitude and explained variance of sinusoid fits, based on the notion that a sinusoidal pattern with large amplitude and a given goodness of fit is more meaningful than a sinusoidal pattern with the same goodness of fit but small amplitude. We used relevance values for second-level statistical analyses. 
Statistical significance of relevance values was primarily tested via permutation tests. In each iteration (1000 iterations), always per participant and per tACS session, the phase condition labels of individual MEP trials were randomly shuffled. Then (randomly ordered) phase bin averages were recalculated and normalized as before. In a first group analysis, individual permuted MEP patterns were averaged over participants to obtain permuted group MEP patterns to which one-cycle sinusoids were fit and relevance values were obtained. The non-permuted (actually obtained) relevance values for alpha/beta/sham conditions were compared to the distributions of permuted relevance values to obtain P-values (i.e. proportion of permuted relevance values higher than the non-permuted relevance value). Secondly, to rule out that the observed group-level effects were driven by a minor subset of the subject sample, we performed a cross-validation group analysis for each tACS session. In this analysis, once for each participant, a one-cycle sinusoid was fit to the group-averaged MEP pattern excluding that one participant. The phase of the best-fitting sinusoid on this sub-group average result was extracted, and a sinusoid with the same fixed phase (but free amplitude) was fitted to the data of the one excluded participant, yielding a relevance value for that fit. This was repeated for each participant, to obtain an average relevance value for the group. This process was again statistically evaluated in a 1000-iteration permutation test.

These group analyses have the benefit of statistical power, since individual participants' results are averaged into a group result. But they are based on the assumption that, in tACS-TMS, putative sinusoidal patterns of MEPs over phase bins are phase-locked. Randomly phaseshifted individual results could cancel each other out. Additionally, caution should be taken when interpreting the generalizability of the group curve-fitting results, as the curve fitting approach used here corresponds to a fixed effects analysis. For this reason we additionally performed an individual participant analysis. Per tACS session we obtained relevance values from best-fitting sinusoids on individual patterns of MEPs over phase bins. We obtained null distributions (1000 iterations) for each participant/session separately, extracting median permuted relevance values from each. As the data was found to be not normally distributed (according to the Kolmogorov-Smirnov test), the Wilcoxon signed-rank test was performed on the group level, testing observed individual relevance values against associated medians from individual null distributions. Family wise Bonferroni error corrections were applied to each of the separate tests $\left(3\right.$ comparisons per test, $\alpha_{\text {bonf }}=0.05 / 3$ ).

\section{Post hoc analysis: high versus low beta peaks}

After inspection of the individual beta frequencies obtained in the EEG localizer, we noticed a large distribution of individual peak frequencies within the beta band (between 16 and 25Hz) and performed a median split on individual beta frequencies to divide the sample into two groups with either low- $(<19 \mathrm{~Hz})$ or high-frequency $(>19 \mathrm{~Hz})$ beta peaks (see table 1 for observed individual peak alpha and beta frequencies per participant). Subsequently, we performed post-hoc analyses in which the data was split into two groups, one with a low $(\mathrm{N}=7$, mean frequency $=16.5( \pm 0.75))$ and one with a high $(N=8$, mean frequency $=21.3( \pm 2.2))$ individual peak beta frequency. All analyses performed on the group level were repeated for these separate groups.

Table 1. Overview of the values obtained in the individual alpha and beta EEG localizers per participant that were used for tACS entrainment. 


\begin{tabular}{|rrr|}
\hline Participant & Individual Alpha & Individual Beta \\
\hline 1 & 10.6 & 23.5 \\
\hline 2 & 11.4 & 20 \\
3 & 10.2 & 21.25 \\
\hline 4 & 10 & 16 \\
5 & 8.6 & 18 \\
\hline 6 & 10.6 & 19 \\
7 & 8 & 16 \\
\hline 8 & 9.4 & 21 \\
9 & 11.8 & 16.5 \\
\hline 10 & 10.8 & 16.75 \\
\hline 11 & 10.2 & 25 \\
\hline 12 & 10.2 & 16 \\
13 & 10.2 & 16 \\
\hline 14 & 11.4 & 19.5 \\
15 & 9.8 & 19.5 \\
\hline
\end{tabular}

\section{Results}

We first evaluated the causal effect of tACS at individual alpha and beta frequency on corticospinal excitability by comparing average MEPs before, during and after tACS administration (Figure 2). The repeated measures ANOVA with factors time (pre/during/post) and condition (alpha/beta/sham) revealed a significant main effect of time $(\mathrm{F}(2,28)=7.64, \mathrm{p}<.01)$, but no significant main effect of condition $(\mathrm{F}(2,28)=.58, p=.56)$ or time*condition interaction $(\mathrm{F}(4,56)=1.04, \mathrm{p}=.39)$. Bonferroni corrected pairwise comparisons revealed significant differences between both the pre and during $(p<.001)$, and a nearly significant difference between the pre and post measurement $(p=.05)$, showing a general increase of MEP amplitude over time. Results indicate that no frequency-specific increases in MEP amplitude resulted from tACS entrainment (either alpha or beta).

We then looked at the causal role of tACS phase on corticospinal excitability, by evaluating relevance values for one-cycle sinusoid fits to MEPs elicited by TMS at eight equidistant phase bins of ongoing tACS. Group analysis involved sinusoid-fitting to a group average of normalized MEPs, separately for alpha, beta, and sham tACS sessions. Relevance values of these three fits were tested against permutation-based null distributions. These analyses revealed a significant effect for beta $\left(p=.011<\alpha_{\text {bonf }}\right)$, but not alpha $\left(p=.58>\alpha_{\text {bonf }}\right)$ or $\operatorname{sham}\left(p=.11>\alpha_{\text {bonf }}\right)$ tACS, see Figure 3A. The post-hoc analysis for low and high beta groups revealed statistically significant fits in only the low beta group $\left(p=.002<\alpha_{\text {bonf }}\right)$, but not the high beta group $(p=$ $.57>\alpha_{\text {bonf, }}$, see Figure 3B). 


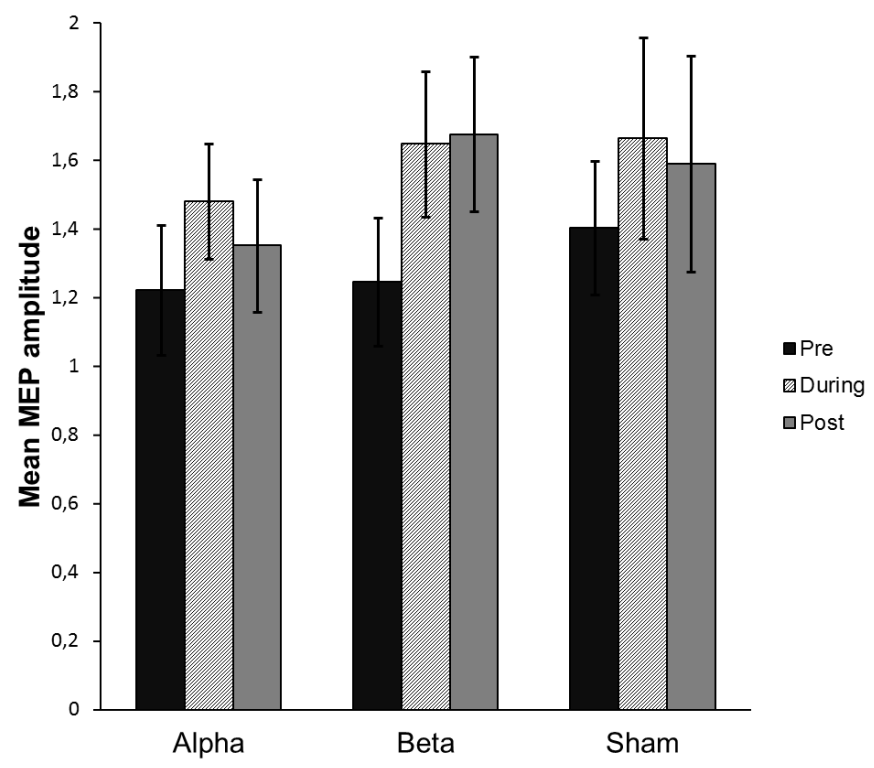

Figure 2. Results of the effect of either individual alpha, individual beta or sham entrainment on mean MEP amplitude.

To rule out that these results were based only on a minor subgroup of participants, we performed a cross-validation analysis (see methods). Results showed no significant effects for alpha $\left(p=.53>\alpha_{\text {bonf }}\right)$, sham $\left(p=.42>\alpha_{\text {bonf }}\right)$, or beta tACS $\left(p=.05>\alpha_{\text {bonf }}\right)$. Again, the post-hoc analysis splitting up high and low beta groups revealed only an effect for the lower beta $(\mathrm{p}<$ $.013<\alpha_{\text {bonf }}$ ), not the higher beta $\left(p=.96>\alpha_{\text {bonf }}\right.$ ), group.

Lastly, we performed an individual participant analysis, meaning that we extracted relevance values, and created null distributions of permuted relevance values, for each participant in each tACS condition. We compared the non-permuted relevance values with medians of those null distributions using the Wilcoxon signed-rank test. This analysis revealed no significant effects for either alpha $\left(T=84, p=0.09>\alpha_{\text {bonf }}\right)$, beta $\left(T=55, p=0.58>\alpha_{\text {bonf }}\right)$ or $\operatorname{sham}(T=65, p$ $\left.=0.40>\alpha_{\text {bonf }}\right)$. For both high and low beta this result was also not significant $(\mathrm{T}=10, \mathrm{p}=$ $0.88>\alpha_{\text {bonf }}$ and $\mathrm{T}=20, \mathrm{p}=0.19>\alpha_{\mathrm{bonf}}$ respectively). 

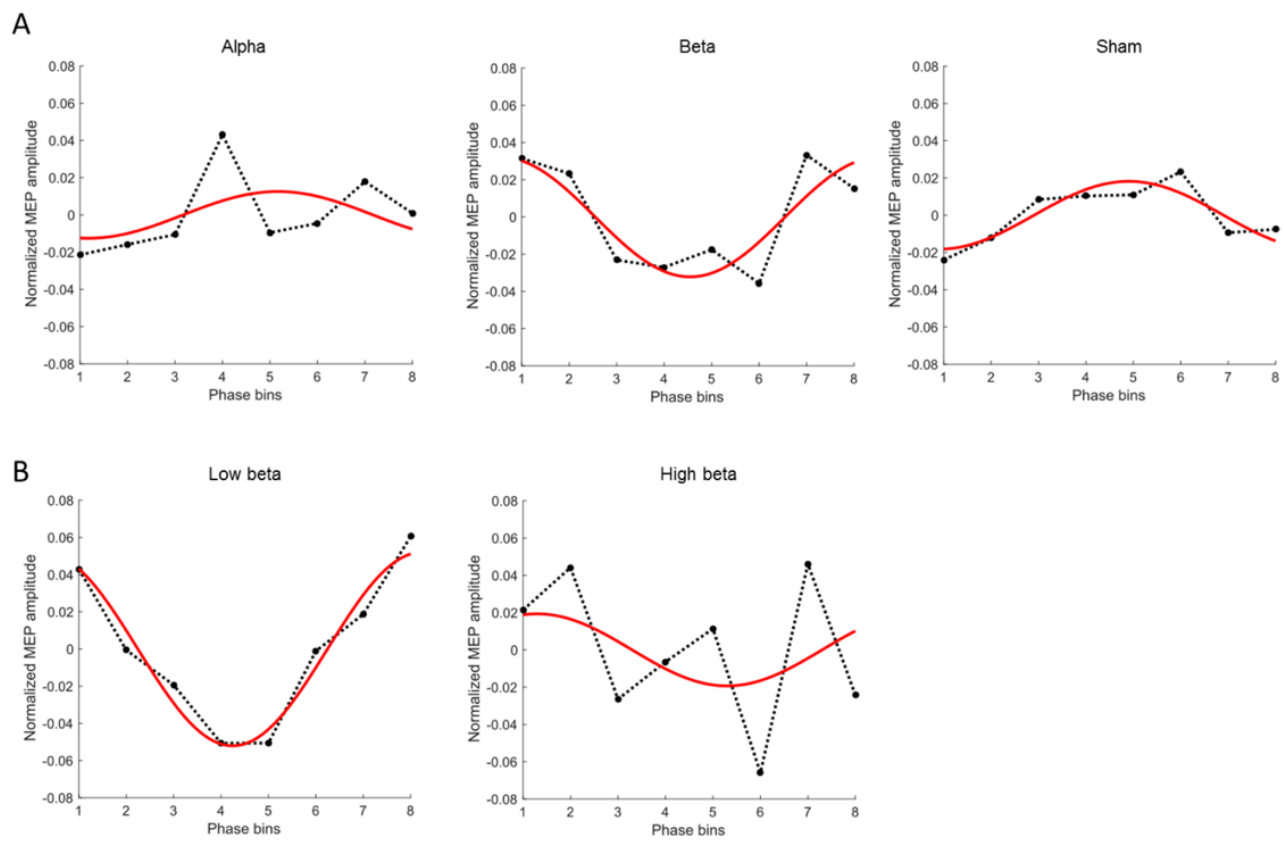

Figure 3. (A) Results of the group curve fitting analysis for alpha, beta, and sham and for (B) the high and low beta subgroups. The solid red lines represent the best fitting one-cycle sinusoid. The dotted black lines represent the mean MEP amplitude at different phases of the entraining tACS signal.

\section{Discussion}

We combined single pulse TMS and EMG with tACS at individual alpha and beta frequencies to examine frequency- and phase-dependent effects of online tACS on corticospinal excitability. We found that the magnitude of MEP amplitude was affected by tACS in a phase-dependent manner for individual peak beta frequency tACS application at the time of the TMS pulse. Specifically, group averaged MEP amplitudes displayed a sinusoidal pattern over sequential phase conditions of online tACS. Moreover, post-hoc analyses suggested that this phase-dependence was specific to individuals with low individual beta frequencies $(16-19 \mathrm{~Hz})$, as opposed to high beta frequencies $(19-25 \mathrm{~Hz})$. These results indicate that corticospinal excitability quantified by MEP amplitudes is dependent on both phase and frequency of the ongoing tACS signal at the dominant intrinsic frequency within the beta band, conceivably the low beta band. We did not find frequency-specific offline effects of tACS.

\section{The effect of tACS at alpha and beta frequency on corticospinal excitability}

For the investigation of general offline and online effects of the simultaneous tACS-TMS paradigm we compared measures of corticospinal excitability acquired before, during and after alpha, beta, and sham tACS. When testing for changes in corticospinal excitability, only a main effect of time was observed, resulting from a general increase of MEP amplitude in all sessions. 
We did not observe a modulatory effect on MEPs during or after tACS-TMS stimulation. This finding is in line with other studies reporting no modulatory effects on MEPs during or after tACS over M1 at (individual) beta or other frequencies (Antal et al., 2008; Wach et al., 2013; Nowak et al., 2017).

In contrast, yet other studies have reported such modulatory effects (Schutter \& Hortensius, 2011; Feurra et al., 2011; Feurra et al., 2013), however their design differed to that of the current study. We applied tACS in combination with TMS at individual peak frequencies, for a much longer duration (36.5 minutes) and with the stimulation intensity for tACS being $50 \%$ higher $(1.5 \mathrm{~mA})$. Moreover, we compared stimulation effects to sham effects and found no difference between both stimulation protocols. These differences in the application of tACS might explain the discrepancy between results. Additionally, Pellicciari and colleagues recently showed that administering single TMS pulses to motor cortex has cumulative effects that result in increased MEP amplitudes for both fixed and randomized intervals (Pellicciari, Miniussi, Ferrari, Koch, \& Bortoletto, 2016). Although the inter pulse interval in the current experiment was longer compared to the aforementioned study ( 6.75 seconds compared to four seconds on average), it may still be possible that such cumulative TMS stimulation effects overruled any possible modulatory effect of tACS application on MEP amplitudes during and after tACS. In line with our experimental design and results, Raco and colleagues showed that combining beta-tACS with single pulse TMS had cumulative effects on MEP amplitude exclusively when applied at 120\% MT (Raco, Bauer, Norim, \& Gharabaghi, 2017). Therefore, we cannot rule out that tACS application alone could have frequency-specific modulatory effects on MEP amplitudes.

\section{The effect of tACS phase on corticospinal excitability}

Our results suggest that corticospinal excitability is modulated by ongoing tACS in a phasedependent manner. Average MEP amplitudes elicited at eight equidistant tACS phases displayed a sinusoidal pattern when tACS was applied at individual beta frequencies, but not at individual alpha frequencies or when sham-tACS was applied. This shows that MEP amplitude is dependent on the phase of ongoing individually-calibrated beta-frequency tACS. Our findings are in line with previous studies that reported fixed frequency beta tACS $(20 \mathrm{~Hz})$ phasedependent TMS-induced MEP amplitude modulations (Raco et al. 2016), but failed to find such effects at non-motor resonance frequencies (Guerra et al., 2016; Nakazono et al., 2016). Moreover, in contrast to previous studies, our experimental design (wide range of measured phases) and analyses (sinusoidal curve fitting) could directly show, for the first time, an oscillatory pattern of acquired MEPs. This supports a direct and continuous influence of beta tACS phase on corticospinal excitability. Moreover, post-hoc analyses in which we divided the data based on intrinsic high $(>19 \mathrm{~Hz})$ or low $(<19 \mathrm{~Hz})$ beta frequency, showed that this tACS phasedependence was highly significant in the low, but not the high beta group.

\section{The potential influence of intrinsic natural oscillations on cortical and corticospinal excitability}

Momentary state of spontaneous neuronal oscillations has been related repeatedly to cortical or corticospinal excitability. Several simultaneous TMS-EEG studies suggest that natural oscillatory power and phase affect corticospinal excitability (Schulz, Übelacker, Keil, Müller \& Weisz., 2014; Mäki \& Ilmoniemi, 2010; Lepage, Saint-Amour \& Théoret, 2008; Keil et al., 2014). Schulz and colleagues (2014) reported a negative correlation between MEP amplitude and high pre-pulse beta-band power in the contralateral motor, premotor, parietal and frontal 
areas, as well as in the ipsilateral temporal areas. This finding is in accordance with other studies showing a reduction of MEP amplitudes with increasing beta band power, especially for low beta frequencies $(12-18 \mathrm{~Hz}$ ) (Mäki \& Ilmoniemi, 2010; Lepage, Saint-Amour \& Théoret, 2008). In addition, TMS-induced MEP amplitudes have shown to increase when TMS pulses are applied at either the peak or the trough of the beta oscillation compared to other phase bins (Keil et al. 2014). Negative versus positive peaks of sensorimotor intrinsic neuronal oscillations at alpha frequency ( $\mu$-rhythm) have been associated with high versus low corticospinal excitability states (Zrenner, Desideri, Belardinelli \& Ziemann, 2017). Elevated MEP amplitude has also been associated with an increase of pre-stimulus corticomuscular coherence in alpha and beta frequencies at several motor activity relevant areas. In addition, the combination of low pre-stimulus beta band power along with high alpha band corticomuscular coherence has been related to high MEPs (and vice versa) (Schulz et al., 2014).

\section{Specificity of phase dependence in the low beta frequency range}

The association of corticospinal excitability with beta band tACS frequency is in line with the idea that brain oscillations in the beta frequency are related to the preparation and execution of movement (Neuper \& Pfurtscheller, 2001; Pfurtscheller, 1981). With regard to both limb movement and somatosensory stimulation, beta frequency has been reported to be lower over cortical representations of the hand area (below $20 \mathrm{~Hz}$ ) than of the foot area (above $20 \mathrm{~Hz}$ ) (Neuper \& Pfurtscheller, 2001). This could explain why we found phase dependency especially for lower beta band tACS application, given that MEPs were measured from the FDI muscle of the hand. On the other hand, it is also possible that tACS application at the individual peak frequency from within the low beta band would have resulted in phase-dependent effects in all participants, including those who had a high individual peak beta frequency when tested or the grand beta band. Thus, tACS at low beta frequency could also be beneficial irrespectively of the intrinsic peak beta frequency. Alternatively, it is possible that phase-dependent effects are only present for individuals with an intrinsic low peak beta. Based on our data these possibilities cannot be distinguished.

\section{Individual peak frequencies}

Natural peak frequencies seem to be a crucial factor for cortical and corticospinal excitability. Using rhythmic repetitive TMS (rTMS), Romei et al. (2016) demonstrated that elicited responses are strongest when stimulation is applied at the intrinsic individual peak beta frequency. In addition, tACS at intrinsic peak frequencies of specific frequency bands appears to be an important factor that could play a major role in (beta) entrainment efficacy (Davis, Tomlinson, \& Morgan, 2012).

In the current study, we applied tACS at the intrinsic peak frequency. With respect to previous research on tACS entrainment effects, this could have resulted in the entrainment of the applied frequency in the underlying cortical areas (Antal \& Paulus, 2013; Herrmann, Rach, Neuling \& Strüber, 2013; Fröhlich \& McCormick, 2010; Witkowski et al., 2016). Indeed, entrainment at a frequency that is close to the underlying intrinsic frequency leads to the strongest increase in frequency power (Merlet et al., 2013; Fröhlich \& McCormick, 2010). Thus, by applying tACS at the intrinsic peak frequency it seems reasonable that tACS phase-specific TMS is also associated with the natural peak frequency. tACS-induced modulation of corticospinal excitability could therefore be linked to the frequency and phase of intrinsic neuronal oscillations. Therefore, measuring individual peak beta frequency and linking TMS to both this 
frequency and a particular phase of the tACS signal could be most beneficial for robust and meaningful measures of corticospinal excitability.

\section{Limitations}

In line with our hypothesis, the results of the current experiment showed a striking correspondence of the beta group MEP pattern over eight tACS phase bins to a one-cycle sinusoid. Nonetheless, it is important to also be aware of the limitations of our results. Based on previous research it seems reasonable to hypothesize that tACS leads to an entrainment of the applied frequency oscillation in underlying neuronal networks (Antal \& Paulus, 2013; Herrmann, Rach, Neuling \& Strüber, 2013; Witkowski et al., 2016; Zaehle et al., 2010; Neuling et al., 2013; Fröhlich \& McCormick, 2010). By applying TMS phase-locked to the tACS signal over the primary motor cortex, we could assume that TMS is also phase locked to the entrained underlying neuronal oscillation. Thus, if this were the case, our results would suggest that corticospinal excitability is dependent on the current state of the underlying neuronal oscillation at the time and location of stimulation and that the driving agents in modulating excitability in the motor system are neuronal oscillations in the beta frequency range. However, we did not control for a tACS entrainment effects by simultaneously recording ongoing neuronal oscillation signals. Therefore, we can only draw conclusions about the effects of specific tACS frequencies and phases on TMS-induced MEP measures of corticospinal excitability. Future empirical studies, specifically also assessing tACS entrainment efficacy with EEG, need to be conducted in order to draw conclusions about the causal relevance of the frequency and phase of underlying neuronal oscillations for corticospinal excitability.

We report a general increase of MEP amplitude over time across all three visits (alpha, beta and sham) and conclude that we do not find modulatory effects of tACS at either stimulation frequency. However, we cannot rule out that periodical spTMS (although at jittered inter pulse intervals around seven seconds) enhances corticomuscular coherence, which has previously been demonstrated to have an effect on MEP amplitudes (Schulz et al., 2014). Therefore, the general increase of MEP amplitude may be caused by periodic spTMS-triggered enhanced corticomuscular coherence, thereby overruling any possible modulatory effect of tACS. Thus, a frequency-specific modulatory effect of tACS on MEP amplitude may still exist when tACS is applied without simultaneous TMS.

Curve-fitting in the beta group only showed a trend in the cross validation. Also, neither of the beta groups (all participants or low-beta participants) yielded significant results in the individual participant analysis that fit sinusoids to individual participant data. This asks for caution both when generalizing the results to individual corticospinal excitability assessments and when thinking of applying them to increase reliability of TMS measures of corticospinal excitability.

We determined individual dominant alpha frequency by analyzing resting EEG measures while participants kept their eyes closed. However, tACS at individual alpha peak frequencies was applied when participants kept their eyes open. It is therefore possible that the individual alpha peak frequencies used for tACS do not exactly correspond to the intrinsic peak alpha frequency when eyes are kept open. Moreover, alpha oscillations measured from resting EEG with eyes closed are prominent over posterior cortical areas. Thus, it is possible that the recorded peak alpha frequencies from the central electrode (C3) correspond to neuronal activity from posterior cortical areas instead of neuronal activity from the motor cortex. This might explain why no significant effects were observed in the alpha condition. 


\section{Methodological implication}

In the present study we demonstrate that TMS measures of corticospinal excitability are influenced by time coupling of the TMS pulse with tACS frequency and phase. Previous studies showed that tACS successfully entrains oscillations of underlying neuronal networks. Together, these findings indicate that corticospinal excitability may depend on the phase of the intrinsic peak beta frequency, predominantly on the lower beta frequency band $(16-19 \mathrm{~Hz})$. Most studies investigating corticospinal excitability by measuring TMS-induced MEPs do not control for the state of the brain during stimulation. With respect to previous research and under restriction of the discussed limitations, our findings indicate that study outcomes may be influenced by fluctuations of corticospinal excitability, if the state of the brain is not controlled for, or at least not accounted for, when corticospinal excitability is measured. In addition, it stands to reason that local oscillation phase and frequency at the time of TMS administration may not only be crucial for the investigation of corticospinal excitability, but also for other forms of TMS application, such as motor plasticity inducing stimulation protocols or research on brain regions other than M1. Further investigations of frequency and phase-dependence of TMS efficacy could possibly help to explain recently accented high variability of TMS effects both across and within individuals (Schilberg, Schuhmann \& Sack, 2017) and lead to more robustness of TMS measures. In order to acquire meaningful and reliable results it is important to carefully investigate underlying brain mechanisms that could influence TMS effects and to improve existing or to develop new application techniques. Incorporating measures of functional, physiological and anatomical properties of targeted neuronal networks into TMS protocols introduces a new era of information based approaches of non-invasive transcranial brain stimulation, which aim for enhanced specificity of stimulation (Romei et al., 2016). This is not only crucial for fundamental research, but also for clinical applications of TMS in which high efficacy of applied protocols is necessary for a successful therapy of a disease or the reliable monitoring of its course. 


\section{References}

Antal, A., \& Paulus, W. (2013). Transcranial alternating current stimulation (tACS). Frontiers in Human Neuroscience, 7, 317. DOI: 10.3389/fnhum.2013.00317

Antal, A., Boros, K., Poreisz, C., Chaieb, L., Terney, D., \& Paulus, W. (2008). Comparatively weak aftereffects of transcranial alternating current stimulation (tACS) on cortical excitability in humans. Brain Stimul, 1(2), 97-105. DOI: 10.1016/j.brs.2007.10.001

Bestmann, S., \& Krakauer, J. W. (2014). The uses and interpretations of the motor-evoked potential for understanding behaviour. Exp. Brain Res, 233(3), 679-689. DOI: 10.1007/s00221-014-4183-7

Buzsáki, G., \& Draguhn, A. (2004). Neural Oscillations in Cortical Networks. Science, 304(5679), 19261929. DOI: $10.1126 /$ science. 1099745

Chen, R., Kumar, S., Garg, R. R., \& Lang, A. E. (2001). Impairment of motor cortex activation and deactivation in Parkinson's disease. Clinical Neurophysiology, 112(4), 600-607. DOI: 10.1016/S1388-2457(01)00466-7

Davis, N. J., Tomlinson, S. P., \& Morgan, H. M. (2012). The Role of Beta-Frequency Neural Oscillations in Motor Control. Journal of Neuroscience, 32(2), 403-404. DOI: 10.1523/JNEUROSCI.510611.2012

Di Lazzaro, V., Oliviero, A., Profice, P., Ferrara, L., Saturno, E., Pilato, F., \& Tonali, P. A. (1999). The diagnostic value of motor evoked potentials. Clin Neurophysiol., 110(7), 1297-1307. DOI: $10.1016 /$ S1388-2457(99)00060-7

Di Lazzaro, V., Oliviero, A., Pilato, F., Saturno, E., Dileone, E., Marra, C., ... Tonali, P. A. (2004). Motor cortex hyperexcitability to transcranial magnetic stimulation in Alzheimer's disease. J Neurol Neurosurg Psychiatry, 75, 555-559. DOI: 10.1136/jnnp.2003.018127

Dugué, L., Marque, P., \& VanRullen, R. (2011). The phase of ongoing oscillations mediates the causal relation between brain excitation and visual perception. The Journal of Neuroscience, 31(33), 1188993. DOI: $10.1523 / J N E U R O S C I .1161-11.2011$

Ferreri, F., Pauri, F., Pasqualetti, P., Fini, R., Dal Forno, G., \& Rossini, P. M. (2002). Motor cortex excitability in Alzheimer's disease: A transcranial magnetic stimulation study. Annals of Neurology, 53(1), 102-108. DOI: 10.1002/ana.10416

Feurra, M., Bianco, G., Santarnecchi, E., Del Testa, M., Rossi, A., \& Rossi, S. (2011). Frequency-Dependent Tuning of the Human Motor System Induced by Transcranial Oscillatory Potentials. Journal of Neuroscience, 31(34), 12165-12170. DOI: 10.1523/JNEUROSCI.0978-11.2011

Feurra, M., Pasqualetti, P., Bianco, G., Santarnecchi, E., Rossi, A., \& Rossi, S. (2013). State-Dependent Effects of Transcranial Oscillatory Currents on the Motor System: What You Think Matters. Journal of Neuroscience, 33(44), 17483-17489. DOI: 10.1523/JNEUROSCI.1414-13.2013

Fiebelkorn, I. C., Foxe, J. J., Butler, J. S., Mercier, M. R., Snyder, A. C., \& Molholm, S. (2011). Ready, Set, Reset: Stimulus-Locked Periodicity in Behavioral Performance Demonstrates the Consequences 
of Cross-Sensory Phase Reset. Journal of Neuroscience, 31(27), 9971-9981. DOI: 10.1523/JNEUROSCI.1338-11.2011

Fröhlich, F., \& McCormick, D.A. (2010). Endogenous Electric Fields May Guide Neocortical Network Activity. Neuron, 67(1), 129 - 143. DOI: 10.1016/j.neuron.2010.06.005

Fuhr, P., Borggrefe-Chappuis, A., Schindler, C. \& Kappos, L. (2001). Visual and motor evoked potentials in the course of multiple sclerosis. Brain 124(11), 2162-2168, DOI: 10.1093/brain/124.11.2162

Guerra, A., Pogosyan, A., Nowak, M., Tan, H., Ferreri, F., Di Lazzaro, V., \& Brown, P. (2016) Phase Dependency of the Human Primary Motor Cortex and Cholinergic Inhibition Cancelation During Beta tACS. Cereb Cortex, 26(10), 3977-3990. DOI: 10.1093/cercor/bhw245

Herrmann, C. S., Rach, S., Neuling, T., \& Strüber, D. (2013). Transcranial alternating current stimulation: a review of the underlying mechanisms and modulation of cognitive processes. Front Hum Neurosci, 7(279). DOI: 10.3389/fnhum.2013.00279

Jensen, O., \& Mazaheri, A. (2010). Shaping functional architecture by oscillatory alpha activity: gating by inhibition. Frontiers in Human Neuroscience, 4(186). DOI: 10.3389/fnhum.2010.00186

Jung, N. H., Delvendahl, I., Kuhnke, N. G., Hauschke, D., Stolle, S., \& Mall, V. (2010). Navigated transcranial magnetic stimulation does not decrease the variability of motor-evoked potentials. Brain Stimulation, 3(2), 87-94. DOI: 10.1016/j.brs.2009.10.003

Jurkiewicz, M. T., Gaetz, W. C., Bostan, A. C., \& Cheyne, D. (2006). Post-movement beta rebound is generated in motor cortex: Evidence from neuromagnetic recordings. NeuroImage, 32(3), 1281 1289. DOI: $10.1016 /$ j.neuroimage.2006.06.005

Kallmann, B. A., Fackelmann, S., Toyka, K. V., Riekmann, P., \& Reiners, K. (2006). Early abnormalities of evoked potentials and future disability in patients with multiple sclerosis. Mult Scler., 12(1), 58-65. DOI: $10.1191 / 135248506 \mathrm{~ms} 1244 \mathrm{oa}$

Keil, J., Timm, J., Sanmiguel, I., Schulz, H., Obleser, J., \& Schönwiesner, M. (2014). Cortical brain states and corticospinal synchronization influence TMS-evoked motor potentials. Journal of Neurophysiology, 111(3), 513-9. DOI: 10.1152/jn.00387.2013

Kiers, L., Cros, D., Chiappa, K. H., \& Fang, J. (1993). Variability of motor potentials evoked by transcranial magnetic stimulation. Electroencephalography and Clinical Neurophysiology/ Evoked Potentials, 89(6), 415-423. DOI: 10.1016/0168-5597(93)90115-6

Kilavik, B., Zaepffel, M., Brovelli, A., Mackay, W., \& Riehle, A. (2013). The ups and downs of beta oscillations in sensorimotor cortex. Experimental Neurology, 245, 15-26. DOI: 10.10167j.expneurol.2012.09.014

Lefaucheur, J. (2005). Motor cortex dysfunction revealed by cortical excitability studies in Parkinson's disease: influence of antiparkinsonian treatment and cortical stimulation. Clinical Neurophysiology, 116(2), 244-253. DOI: 10.1016/j.clinph.2004.11.017

Lepage, J. F., Saint-Amour, D., \& Théoret, H. (2008). EEG and neuronavigated single-pulse TMS in the 108 
study of the observation/execution matching system: are both techniques measuring the same process? J Neurosci Methods, 175(1), 17-24. DOI: 10.1016/j.jneumeth.2008.07.021

Mäki, H., \& Ilmoniemi, R. J. (2010). EEG oscillations and magnetically evoked motor potentials reflect motor system excitability in overlapping neuronal populations. Clinical Neurophysiology, 121(4), 492-50. DOI: 10.1016/j.clinph.2009.11.078

Mathewson, K.E., Lleras, A., Beck, D.M., Fabiani, M., Ro, T., \&Gratton, G. (2011). Pulsed out of awareness: EEG alpha oscillations represent a pulsed-inhibition of ongoing cortical processing. Frontiers in Psychology, 2(99), 1-15. DOI: 10.3389/fpsyg.2011.00099

Merlet, I., Birot, G., Salvador, R., Molaee-Ardekani, B., Mekonnen, A., Soria-Frish, A., Ruffini, G., Miranda, P.C., \& Wendeling, F. (2013). From oscillatory transcranial current stimulation to scalp EEG changes: a biophysical and physiological modeling study. PLOS ONE, 8(2), DOI: 10.1371/journal.pone. 0057330

Moliadze, V., Fritzsche, G., \& Antal, A. (2014). Comparing the Efficacy of Excitatory Transcranial Stimulation Methods Measuring Motor Evoked Potentials. Neural Plasticity, 2014(837141). DOI: 10.1155/2014/837141

Nakazono, H., Ogata, K., Kuroda, T., \& Tobimatsu, S. (2016) Phase and Frequency-Dependent Effects of Transcranial Alternating Current Stimulation on Motor Cortical Excitability. PLOS ONE, 11(9), e0162521. DOI: 10.1371/journal.pone.0162521

Neuling, T., Rach, S., \& Herrmann, C.S. (2013). Orchestrating neuronal networks: sustained after-effects of transcranial alternating current stimulation depend upon brain states. Frontiers in Human Neuroscience, 7:161. DOI: 10.3389/fnhum.2013.00161

Neuper, C., \& Pfurtscheller, G. (2001). Evidence for distinct beta resonance frequencies in human EEG related to specific sensorimotor cortical areas. Clinical Neurophysiology, 112(11), 2084-2097. DOI: 10.1016/S1388-2457(01)00661-7

Ni, Z., \& Chen, R. (2015). Transcranial magnetic stimulation to understand pathophysiology and as potential treatment for neurodegenerative diseases. Translational Neurodegeneration, 4, 22. DOI: $10.1186 /$ s40035-015-0045-x

Niedermeyer, E. (2005). The normal EEG of the waking adult. Electroencephalography: basic principles, clinical applications and related field. Baltimore: Lippincott, Williams \& Wilkins. 167, 155-164.

Nowak, M., Hinson, E., van Ede, F., Pogosvan, A., Guerra, A., Quinn, A., Brown, P., \& Stagg, C. J. (2017). Driving Human Motor Cortical Oscillations Leads to Behaviorally Relevant Changes in Local GABA(A) Inhibition: A tACS-TMS Study. The Journal of Neuroscience 37(17), 4481-4492. DOI: 10.1523/JNEUROSCI.0098-17.2017

Oostenveld, R., Fries, P., Maris, E., \& Schoffelen, J. M. (2011). FieldTrip: Open Source Software for Advanced Analysis of MEG, EEG, and Invasive Electrophysiological Data. Computational Intelligence and Neuroscience, 2011(156869), 1-9. DOI: 10.1155/2011/156869

Pascual-Leone, A., Tormos, J., \& Keenan, J. (1998). Study and modulation of human cortical excitability 
with transcranial magnetic stimulation. Journal of Clinical Neurophysiology. 15(4), 333-343.

Pellicciari, M. C., Miniussi, C., Ferrari, C., Koch, G., \& Bortoletto, M. (2016). Ongoing cumulative effects of single TMS pulses on corticospinal excitability: An intra- and inter-block investigation. Clinical Neurophysiology, 127(1), 621-628. DOI: 10.1016/j.clinph.2015.03.002.

Pfurtscheller, G. (1981). Central beta rhythm during sensorimotor activities in man. Electroencephalography and Clinical Neurophysiology, 51(3), 253-264. DOI: 10.1016/0013-4694(81)90139-5

Pfurtscheller, G., Stancák, A., \& Neuper, C. (1996). Post-movement beta synchronization. A correlate of an idling motor area? Electroencephalography and Clinical Neurophysiology, 98(4), 281-293. DOI: 10.1016/0013-4694(95)00258-8

Raco, V., Bauer, R., Norim, S., \& Gharabaghi, A. (2017). Cumulative effects of single TMS pulses during beta-tACS are stimulation intensity-dependent. Brain Stimulation, 10(6), 1055-1060. DOI: 10.16/j.brs.2017.07.009

Raco, V., Bauer, R., Tharsan, S., \& Gharabaghi, A. (2016). Combining TMS and tACS for Closed-Loop Phase-Dependent Modulation of Corticospinal Excitability: A Feasibility Study. Frontiers in Cellular Neuroscience, 10(143), 1-8. DOI: 10.3389/fncel.2016.00143

Rico, A., Audoin, B., Frangues, J., Eusebio, A., Reuter, F., Malikova, I., ... Attarian, S. (2009). Motor evoked potentials in clinically isolated syndrome suggestive of multiple sclerosis. Mult. Scler., 15(3), 355-362. DOI: $10.1177 / 1352458508099612$

Romei, V., Bauer, M., Brooks, J. L., Economides, M., Penny, W., Thut, G., ... Bestmann, S. (2016). Causal evidence that intrinsic beta-frequency is relevant for enhanced signal propagation in the motor system as shown through rhythmic TMS. NeuroImage, 126, 120-130. DOI: 10.1016/j.neuroimage.2015.11.020

Romei, V., Thut, G., \& Silvanto, J. (2016). Information-Based Approaches of Noninvasive Transcranial Brain Stimulation. Trends in Neuroscience, 39(11), 782-795. DOI: 10.1016/j.tins.2016.09.001

Rösler, K. M., Roth, D. M., \& Magistris, M. R. (2008). Trial-to-trial size variability of motor-evoked potentials. A study using the triple stimulation technique. Experimental Brain Research, 187(1), 5159. DOI: $10.1007 / \mathrm{s} 00221-008-1278-\mathrm{z}$

Rossi, S., Hallett, M., Rossini, P. M., \& Pascual-Leone, A. (2012). Safety, ethical considerations, and application guidelines for the use of transcranial magnetic stimulation in clinical practice and research. Clinical Neurophysiology, 120(12), 323-330. DOI: 10.1016/j.clinph.2009.08.016.Rossi

Rossini, P. M., \& Rossi, S. (1998). Clinical applications of motor evoked potentials. Clinical Neurophysiology, 106 (3), 180-194. DOI: 10.1016/S0013-4694(97)00097-7.

Rothwell, J. C., Hallett, M., Berardelli, A., Eisen, A., Rossini, P., \& Paulus, W. (1999). Magnetic stimulation: motor evoked potentials. The International Federation of Clinical Neurophysiology. Electroencephalography and Clinical Neurophysiol Suppl, 52, 97-103.

Schilberg, L., Schuhmann, T., \& Sack, A. T. (2017). Interindividual Variability and Intraindividual 110 
Reliability of Intermittent Theta Burst Stimulation-induced Neuroplasticity Mechanisms in the Healthy Brain. Journal of Cognitive Neuroscience, 29(6), 1022-1032. DOI: 10.1162/jocn_a_01100

Schulz, H., Übelacker, T., Keil, J., Müller, N., \& Weisz, N. (2014). Now I am ready - Now i am not: The influence of pre-TMS oscillations and corticomuscular coherence on motor-evoked potentials. Cerebral Cortex, 24(7), 1708-1719. DOI: 10.1093/cercor/bht024

Schutter, D. J. L. G., \& Hortensius, R. (2011). Brain oscillations and frequency-dependent modulation of cortical excitability. Brain Stimulation, 4(2), 97-103. DOI: 10.1016/j.brs.2010.07.002

Silvanto, J., \& Pascual-Leone, A. (2008). State-Dependency of Transcranial Magnetic Stimulation. Brain Topography, 21(1), 1-10. DOI: 10.1007/s10548-008-0067-0

Ten Oever, S., de Graaf, T. A., Bonnemayer, C., Ronner, J., Sack, A. T., \& Riecke, L. (2016). Stimulus Presentation at Specific Neuronal Oscillatory Phases Experimentally Controlled with tACS: Implementation and Applications. Frontiers in Cellular Neuroscience, 10(240), 1-8. DOI: 10.3389 /fncel.2016.00240

Thut, G., Northoff, G., Ives, J. R., Kamitani, Y., Pfennig, A., Kampmann, F., Schomer, D. L., \& PascualLeone, A. (2003). Effects of single-pulse transcranial magnetic stimulation (TMS) on functional brain activity: a combined event-related TMS and evoked potential study. Clinical Neurophysiology, 114(11), 2071-2080. DOI: 10.1016/S1388-2457(03)00205-0

Wach, C., Krause, V., Moliadze, V., Paulus, W., Schnitzler, A., \& Pollok, B. (2013). Effects of 10 Hz and $20 \mathrm{~Hz}$ transcranial alternating current stimulation (tACS) on motor functions and motor cortical excitability. Behavioural Brain Research, 241, 1-6. DOI: 10.1016/j.bbr.2012.11.038

Witkowski, M., Garcia-Cossio, E., Chander, B. S., Braun, C., Birbaumer, N., Robinson, S. E., \& Soekadar, S. R. (2016). Mapping entrained brain oscillations during transcranial alternating current stimulation (tACS). NeuroImage, 140, 89-98. DOI: 10.1016/j.neuroimage.2015.10.024

Zaehle, T., Rach, S., \& Herrmann, C.S. (2010). Transcranial alternating current stimulation enhances individual alpha activity in human EEG. PLOS ONE, 5(11). DOI: 10.1371/journal.pone.0013766

Zrenner, C., Desideri, D., Belardinelli, P., Ziemann, U. (2017). Real-time EEG-defined excitability states determine efficacy of TMS-induced plasticity in human motor cortex. Brain Stimulation. DOI: 10.1016/j.brs.2017.11.016 



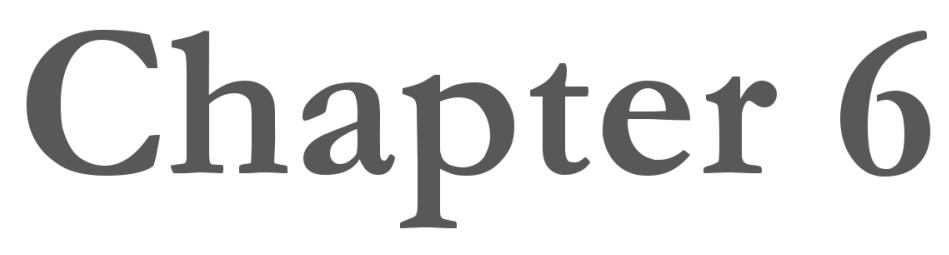

\section{Affective vocalizations influence body ownership as measured in the Rubber Hand Illusion}

Based on:

Engelen, T., Watson, R., Pavani, F., \& de Gelder, B. (2017). Affective vocalizations influ-ence body ownership as measured in the rubber hand illusion. PLoS ONE, 12(10): e0186009. 



\begin{abstract}
Emotional signals, like threatening sounds, automatically ready the perceiver to prepare an appropriate defense behavior. Conjecturing that this would manifest itself in extending the safety zone around the body we used the rubber hand illusion (RHI) to test this prediction. The RHI is a perceptual illusion in which body ownership is manipulated by synchronously stroking a rubber hand and real hand occluded from view. Many factors, both internal and external, have been shown to influence the strength of the illusion, yet the effect of emotion perception on body ownership remains unexplored. We predicted that listening to affective vocalizations would influence how strongly participants experience the RHI. In the first experiment four groups were tested that listened either to affective sounds (angry or happy vocalizations), non-vocal sounds or no sound while undergoing synchronous or asynchronous stroking of the real and rubber hand. In a second experiment three groups were tested comparing angry or neutral vocalizations and no sound condition. There was a significantly larger drift towards the rubber hand in the emotion versus the no emotion conditions. We interpret these results in the framework that the spatial increase in the RHI indicates that under threat the body has the capacity to extend its safety zone.
\end{abstract}

\title{
Introduction
}

Emotional expressions are powerful triggers that at the same time communicate affective information, while also requiring situation-appropriate responses. Research has established the existence of a tight link between emotions and our bodies, and it is clear that perception of emotion stimuli in the environment prepare our bodies for action. This has been documented for the perception of emotional faces (Schutter, Hofman, \& Van Honk, 2008), bodies (de Gelder, Snyder, Greve, Gerard, \& Hadjikhani, 2004; Engelen, de Graaf, Sack, \& de Gelder, 2015; Huis In 't Veld, Van Boxtel, \& de Gelder, 2014), and scenes (Hajcak et al., 2007; van Loon, van den Wildenberg, van Stegeren, Hajcak, \& Ridderinkhof, 2010), as well as sounds conveying affective information (Komeilipoor, Pizzolato, Daffertshofer, \& Cesari, 2013). Especially when confronted with threatening information in our direct vicinity, we can expect physiological responses like a change in heart rate (Roelofs, Hagenaars, \& Stins, 2010), skin conductance, pupil size (Bradley, Miccoli, Escrig, \& Lang, 2008), and pre-activation of our muscles (Huis In 't Veld et al., 2014). This readiness for action is not only reflected in the body, but also in the brain, where it becomes apparent that motor cortex becomes more easily excitable (Borgomaneri, Vitale, Gazzola, \& Avenanti, 2015), and areas involved in action preparation become active (Engelen et al., 2015).

One important relation to explore is the one between action in response to emotions and body ownership. One way of testing changes in body ownership is with the so called Rubber Hand Illusion (RHI). The RHI presents a fascinating phenomenon in which synchronous visuo-tactile stimulation of a rubber hand and tactile stimulation of the real hand can lead to a person misplacing the location of their real hand. This misplacement is evident in a drift of the perceived location of the real hand towards the rubber hand, as well as in the subjective experience of the rubber hand being one's own (Botvinick \& Cohen, 1998). The illusion has been widely used to study the malleability of body ownership and reveals how, in this particular occurrence of multimodal integration, there is a strong dominance of the visual domain over touch and proprioception, possibly related to the fact that vision has far higher spatial acuity. Initial findings have since been extended and a multitude of factors have been found that seem to 
influence the strength of the illusion (Morgan et al., 2011; Pavani, Spence, \& Driver, 2000; Tieri, Tidoni, Pavone, \& Aglioti, 2015; Tsakiris, Tajadura-Jiménez, \& Costantini, 2011) and even transfer of the phenomenon to other body parts (Flögel, Kalveram, Christ, \& Vogt, 2015; Sforza, Bufalari, Haggard, \& Aglioti, 2010) or virtual hands (IJsselsteijn, de Kort, \& Haans, 2006).

Even though in their own right, the concepts of action preparation in response to emotions and conditions for ownership in the RHI have been widely studied, it has not been directly tested yet how emotions can influence our sense of body ownership. The current study sets out to probe the influence of affective vocalizations on the RHI. We used a between-subject design, in which the different groups were exposed to one of four sound conditions (happy vocal, angry vocal, non-vocal or no sounds) while experiencing the illusion. Each participant performed both asynchronous and synchronous stroking trials during the session, and both proprioceptive drift and a questionnaire on the subjective experience of ownership of the rubber hand were used to measure the strength of the illusion. To further disentangle effects between affective and neutral vocalizations, and to exclude the possibility that hearing voices per se can change the strength of the illusion, a second experiment was conducted. The set-up for the second experiment was similar as for the first; however, the groups were either presented with angry vocalizations, neutral vocalizations or no sounds as a control group. We hypothesized that emotional content of the vocalizations, and not neutral vocalizations or sounds that are of neutral content, will alter the strength of the illusion.

\section{Methods}

\section{Participants}

A group of 208 healthy Caucasian volunteers participated in this study $(\mathrm{N}=114$ in experiment $1(90$ female, mean age $(\mathrm{SD})=22.5(3)), \mathrm{N}=94$ in experiment $2(69$ female, mean age $(\mathrm{SD})=$ 23.4(5), minimum age for participation is 18). All participants were right handed and had normal or corrected to normal vision. Participants were unaware of the goal of the study until after the completion of the experiment. The study was performed in accordance to the Declaration of Helsinki and approved by the local ethical committee of Maastricht University. Written informed consent was obtained from all participants. Seven participants were excluded from the analysis because their average proprioceptive drift determined them as outliers in their group by deviating more than 2.5 standard deviations from the mean. For the analysis the following group sizes were included; 29 participants in the happy vocalizations group, 27 participants in the angry vocalization group, 27 in the no sounds group, and 28 in the non-vocal sounds group. For experiment 2 the distribution was as follows; 30 in the no sound group, 31 in the angry vocalizations group and 29 in the neutral vocalization group.

\section{Sound stimuli}

Stimuli consisted of different sound categories; angry vocal, happy vocal, or non-vocal sounds in experiment 1 and additionally neutral vocal in experiment 2 . Brief $(<2$ seconds), affective (angry or happy) or neutral bursts (vocalizations) from females and males of different identities ( 5 males and 5 females), taken from the Montreal Affective Voices database (Belin, FillionBilodeau, \& Gosselin, 2008), were compiled together to create separate sound clips each lasting 1.5 minutes long. 
A condition of non-vocal sounds was included to ensure any observed effect of emotion was not induced by sounds in general and a no sound condition functioned as a baseline measure of the rubber hand illusion. This equivalent clip was made up of non(human)-vocal sounds (e.g., environmental, industrial, animal) taken from the voice localizer described in (Belin et al., 2008).

\section{Experimental set-up}

Participants were seated behind a table on which a raised platform was positioned (measurements $(\mathrm{LxWxH})$ 80x40x10cm). The participant was asked to place their right hand at the right side of the platform; ten $\mathrm{cm}$ to the left of their hand was a divider which blocked the view of their real hand. Ten $\mathrm{cm}$ to the left of the divider a realistic silicone hand was placed, on which they were asked to focus their attention during each trial. Before the start of the experiment participants were provided with over-ear headphones. In the angry vocal, happy vocal or nonvocal sound conditions, sounds were played through the headphones. In the no-sound condition, the headphones remained on the head but no auditory stimulation was delivered. Sound intensity was fixed to the same level for each participant by setting the intensity to a fixed volume on the laptop operated by the experimenter to play the sounds. Participants were asked to rest their left hand underneath the raised platform. After each trial participants were asked to close their eyes and use their left hand to indicate the perceived position of their real hand on a tape measure attached at the end of the platform facing the experimenter. Before this measure of proprioceptive drift was taken, the experimenter positioned the index finger of the participants' left hand at the left end of the platform at varying positions, to rule out any learning effects. The position of the real hand was aligned with $0 \mathrm{~cm}$ on the tape measure, and the number of $\mathrm{cm}$ they deviated from 0 was noted by the experimenter. This was repeated twice after each trial, and the average of these two observations was used as the proprioceptive drift of the participant in that particular trial. During each trial the participant focused their attention on the rubber hand, while both their real hand and the rubber hand were stroked either synchronously or asynchronously by the experimenter. During the asynchronous trials the stroking of the real and the rubber hand was temporally incongruent. Each trial lasted 1.5 minutes, during the entirety of which a sound stimulus was played, if the participant was in one of the sound groups. See figure 1 for a schematic overview of the experimental set-up.

To address some of the shortcomings in experiment 1 (see discussion), a follow up experiment was conducted in which the procedure was slightly altered. To address any possible carry over effects, the real hand of the participant was revealed between each trial. Additionally, paint brushes were used to stroke the hands. To assure that any differences between conditions observed in experiment 1 were not only due to the sounds including voices or not, experiment 2 included a condition with neutral voices. Other than these differences the procedure was the same as in the first experiment. 


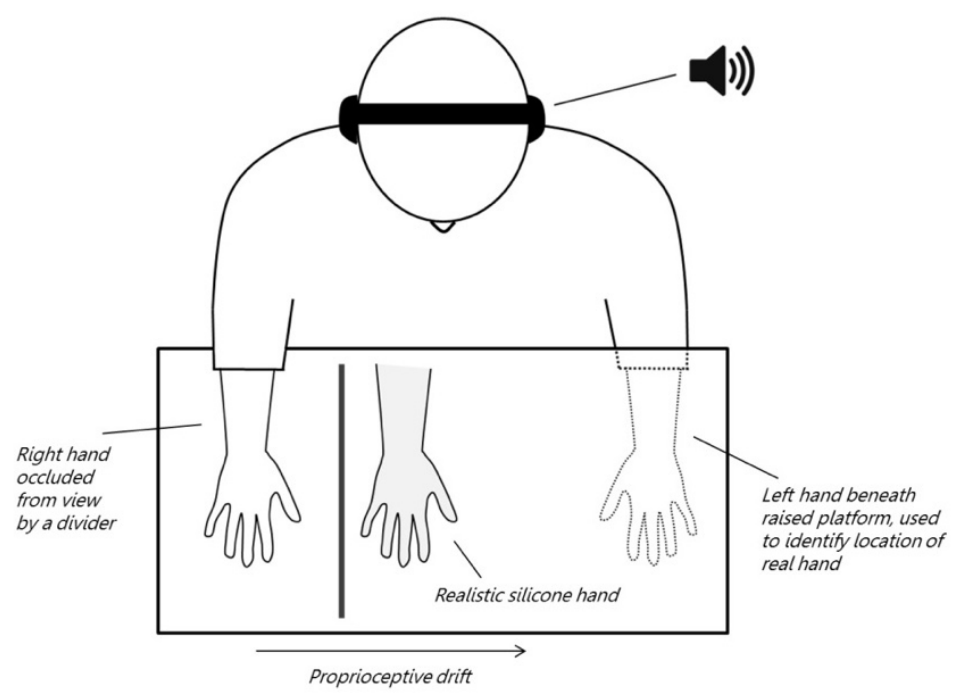

Figure 1. Experimental set-up. The participant is seated at a table on which a raised platform is placed. The participant's right hand is placed on top of the platform, but occluded from view by a divider. The left hand is placed below the platform. During the experiment, participants focus their attention on a realistic silicone hand placed in front of them, while their real hand and the fake hand are brushed by the experimenter in either a synchronous or asynchronous fashion. At the end of each trial, participants use their left hand to indicate where they think their right index finger is located, by moving their left index finger over a tape measure placed on the platform on the side of the experimenter. Additionally, they answered nine questions related to their subjective experience during the trial.

\section{Design}

Each participant performed six trials during the session, three asynchronous and three synchronous. After each trial the strength of the illusion was measured by assessing proprioceptive drift towards the rubber hand and a questionnaire as devised by Botvinick \& Cohen (1998)(see table 1 for the nine questions). Participants were randomly assigned to one of the four conditions (angry, happy, non-vocal or no sound in experiment 1 or angry, neutral or no sound in experiment 2) and the order of questionnaire questions and synchronous or asynchronous trials was counterbalanced within participants.

\section{Analysis}

For each participant the average proprioceptive drift was calculated separately for synchronous and asynchronous trials. Using this average drift as a dependent variable, a mixed ANOVA was run with the stroking condition (synchronous/asynchronous) as a within-subjects factor and sound condition (angry vocal/happy vocal/non-vocal/no-sound in experiment 1; no sound, angry vocal and neutral vocal in experiment 2) as a between-subjects factor. 
As the data from the questionnaire were not normally distributed, WS and BS effects were separately assessed using non-parametric tests. To see how the BS factor (sound condition) influenced each question, the WS factor (stroking condition) was averaged and a Kruskal Wallis test was run for each question separately. Post-hoc tests included Mann-Whitney tests for each group compared to the no sound control, which a Bonferroni corrected $p$-value $\left(p_{\text {bonf }}=0.05 / 3\right.$ for experiment 1 and $p_{\text {bonf }}=0.05 / 2$ for experiment 2 ).

To ensure there was a significant difference in the subjective experience of ownership between the synchronous and asynchronous stroking condition in general, we calculated the average score across questions for each stroking condition, and then compared the two using the Wilcoxon signed-rank test, ignoring the BS (sound condition) factor.

Questionnaire

Q1. It seemed as if I were feeling the touch of the finger in the location where I saw the rubber hand touched

Q2. It seemed as though the touch I felt was caused by the finger touching the rubber hand

Q3. I felt as if the rubber hand were my hand

Q4. It felt as if my (real) hand were drifting towards the left (towards the rubber hand)

Q5. It seemed as if I might have more than one right hand or arm

Q6. It seemed as if the touch I was feeling came from somewhere between my own hand and the rubber hand

Q7. It felt as if my (real) hand were turning 'rubbery'

Q8. It appeared (visually) as if the rubber hand were drifting towards the right (towards my hand)

Q9. The rubber hand began to resemble my own (real) hand, in terms of shape, skin tone, freckles or some other visual feature

Table 1. Questionnaire on subjective experience of the rubber hand illusion assessed after each trial

\section{Results}

The effect of emotion on proprioceptive drift towards the rubber hand

\section{Experiment 1}

The results of the mixed ANOVA on average proprioceptive drift showed a significant effect of stroking condition $(F(1,107)=35.483, p<.001)$, with higher proprioceptive drift in synchronous than in asynchronous stroking (respective means 4 and $2.5 \mathrm{~cm}$ ), confirming a successful inducement of the rubber hand illusion when the rubber hand and the real hand are stroked synchronously. However, there was no interaction between stroking condition and sound condition $(F(3,107)=.365, p=.779)$ and also no main effect of sound condition was evident in the data $(F(3,107)=2.209, p=.091)$. See figure $2 \mathrm{~A}$ for an overview of the results. 
A

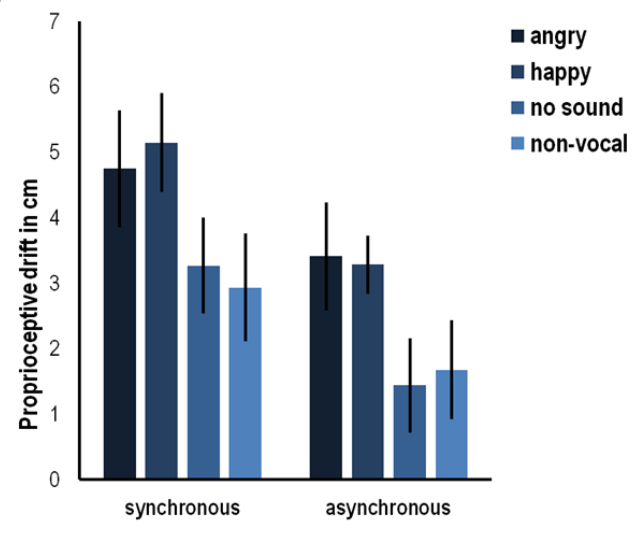

B

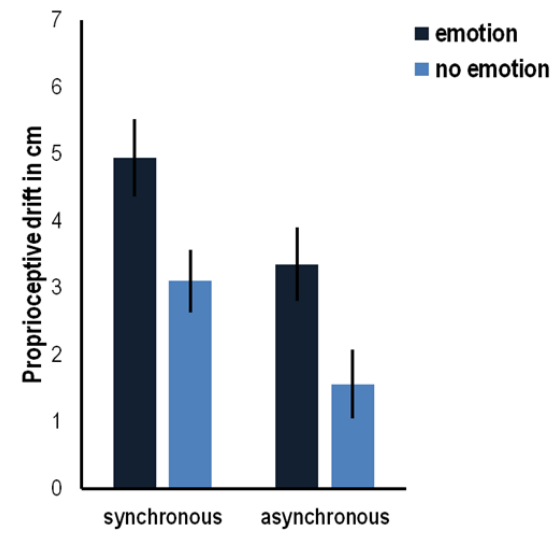

Figure 2. (A) Average proprioceptive drift in $\mathrm{cm}$ in the different conditions of experiment 1 (angry vocal, happy vocal, no sound and non-vocal sounds) in synchronous or asynchronous stroking. Error bars indicate standard error. (B) Average proprioceptive drift in $\mathrm{cm}$ for the data pooled into emotion (angry and happy vocal) and no emotion (no sound and non-vocal sounds) with synchronous or asynchronous stroking. Error bars indicate standard error.

Given the fact we had no a priori hypothesis that valence of the emotion would affect the illusion differently, we decided to pool the groups together into emotion (happy vocal and angry vocal) and no emotion (non-vocal and no sound). The results of this ANOVA revealed again a significant main effect of stroking condition $((F(1,109)=35.480, p<.001)$, as well as a significant main effect of emotion $(F(1,109)=6.728, p=.011)$, which was driven by a higher proprioceptive drift in the emotion versus no emotion conditions (respective means are 4.1 and $2.3 \mathrm{~cm}$ ). There was no interaction between stroking condition and sound condition $(\mathrm{F}(1,109)=.01, p=.92)$. See figure $2 \mathrm{~B}$ for an overview of the results.

\section{Experiment 2}

The results of the mixed ANOVA on average proprioceptive drift measured in experiment 2 showed a significant effect of stroking condition $(F(1,87)=29.510, p<.001)$, as well as a significant main effect of sound condition $(F(2,87)=3.612, p=.031)$. Pairwise comparisons showed that this main effect was driven by trends towards significance in comparisons of no sound versus angry sounds $(p=.083)$ and angry sounds versus neutral sounds $(p=.057)$. No significant difference was apparent between the no sound and neutral sound condition $(p=1)$. There was no significant interaction between stroking condition and sound condition $(F(2,87)$ $=.537, p=.586$ ). See figure 3 for an overview of the results of experiment 2 . 


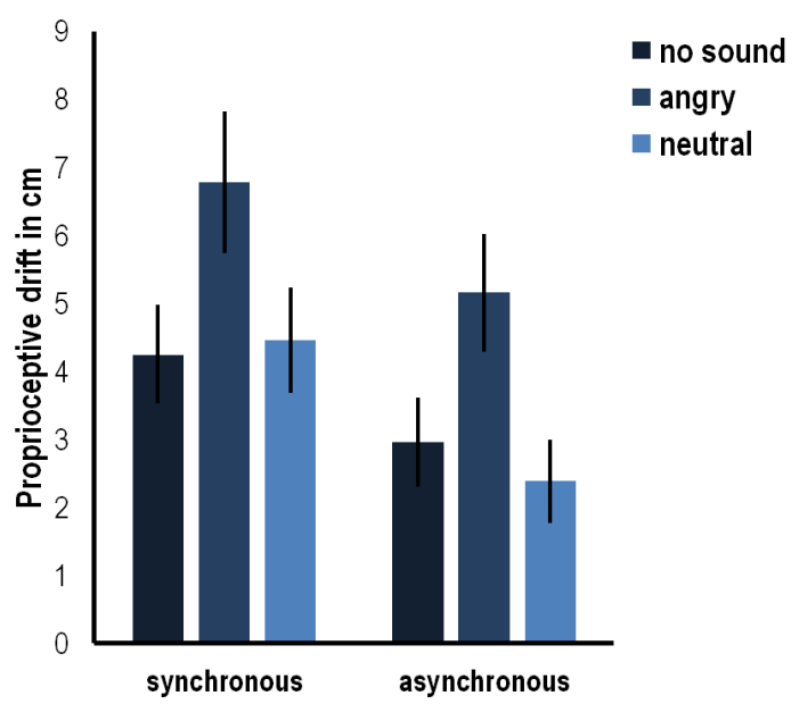

Figure 3. Average proprioceptive drift in $\mathrm{cm}$ in the different conditions of experiment 2 (no sound, angry vocal and neutral vocal) in synchronous and asynchronous stroking. Error bars indicate standard error.

The effect of emotion on the subjective experience of the rubber hand illusion as measured by a questionnaire

In experiment 1, no significant effects of sound condition were found on questions 1-8 (Q1: $H(3)=5.888, p=.117, \mathrm{Q} 2: H(3)=1.351, p=.717$, Q3: $H(3)=1.774, p=.621, \mathrm{Q} 4: H(3)=$ 2.371, $p=.498, \mathrm{Q} 5: H(3)=2.365, p=.500, \mathrm{Q} 6: H(3)=5.643, p=.130, \mathrm{Q} 7: H(3)=3.823, p$ $=.281$, Q8: $H(3)=2.593, p=.459)$, only question 9 showed a significant main effect $(H(3)=$ $8.748, p=.033)$. Bonferroni corrected post-hoc tests for question 9 showed only a significant difference between the happy and no sound condition $\left(U=208.500, p=.007<p_{\text {bonf }}\right)$.

In experiment 2 , there was no significant effect of sound condition reflected in any of the questions $(\mathrm{Q} 1: H(2)=0.714, p=.700, \mathrm{Q} 2: H(2)=1.064, p=.588, \mathrm{Q} 3: H(2)=0.218, p=.897$, Q4: $H(2)=3.177, p=.204$, Q5: $H(2)=0.690, p=.708$, Q6: $H(2)=1.548, p=.461$, Q7: $H(2)$ $=1.404, p=.496, \mathrm{Q} 8: H(2)=3.950, p=.139, \mathrm{Q} 9: H(2)=2.214, p=.331)$.

There was a main WS effect for stroking condition in both experiment $1(z=-9.121, p<.001)$ and experiment $2(z=-7.899, p<.001)$, showing that the subjective experience of ownership was higher for synchronous than asynchronous stroking. See figure 4 for an overview of the questionnaire results for both experiments 1 and 2 . 

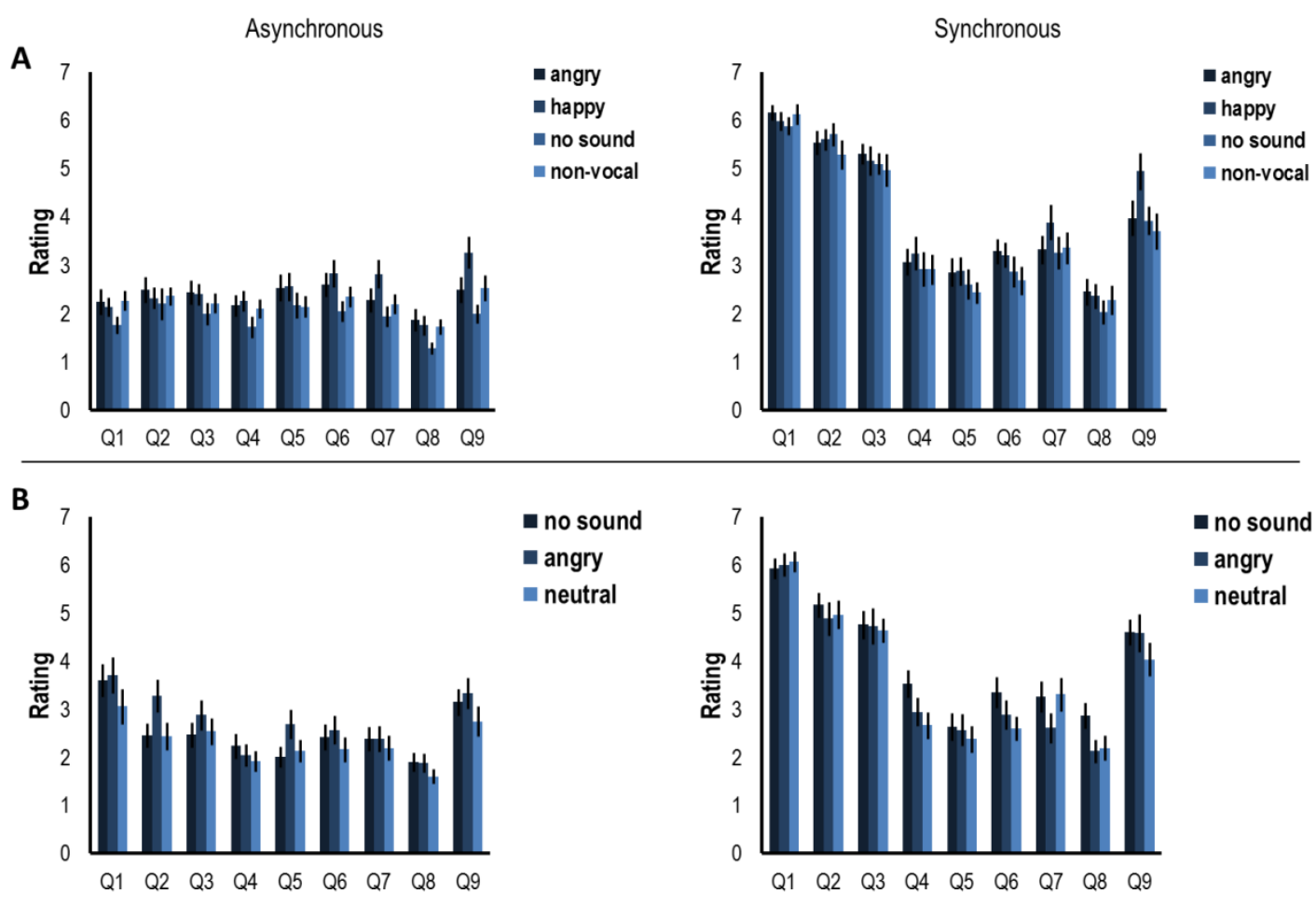

Figure 4. Average scores of the answers to the nine questions related to the subjective strength of the rubber hand illusion as measured in experiment 1 (A) and 2 (B). Error bars indicate standard error.

\section{Discussion}

The current experiment set out to explore the influence of emotional stimuli on body ownership as measured in the rubber hand illusion. Specifically, we investigated if emotional vocalizations would alter the strength of the RHI as compared to a non-vocal and no sounds condition. Our main finding is that passively listening to emotional vocalizations caused a stronger illusion, as measured in the proprioceptive drift towards the rubber hand. This effect was again replicated in experiment 2 , in which we found increased proprioceptive drift caused by angry vocalizations compared to both a no sound and neutral vocalization condition. This also illustrates that it is not the sound of voices per se causing the change in proprioceptive drift, rather the valence of the vocalization. No dissociation could be made between positive and negative vocal sounds. These results demonstrate for the first time that (auditory) emotion perception can influence body ownership and in turn our readiness to accept a rubber hand as our own. The exact mechanism underlying the changes in body ownership in the RHI have been extensively debated, with accounts ranging from sole bottom-up processing (Armel \& Ramachandran, 2003; Botvinick \& Cohen, 1998), to an interoceptive predictive coding model, 
which puts interoceptive inference as a cornerstone of both experienced body ownership and emotion (Seth, 2013). It is by now becoming clear that it is unlikely that the RHI is only driven by bottom-up mechanisms, and the roles of internal representations of the body and current bodily states seem more apparent (Pavani \& Zampini, 2007; Tsakiris, 2010). For the illusion of ownership of the rubber hand to arise it is crucial that the rubber hand is in at least an anatomically plausible position, that the rubber hand is realistic, and stroking of the rubber hand and real hand is synchronous (Tsakiris \& Haggard, 2005). The illusion arises from a manipulation of the timing of multimodal exteroceptive feedback, and both interoceptive and other exteroceptive signals have been shown to influence the illusion.

It is interesting to start exploring body ownership in the light of its evolutionary purpose and what, and if, there actually is any meaning to the fact that in some instances we are more likely to accept something as being part of ourselves. de Vignemont (2017) argues in the 'bodyguard hypothesis' that one function of body ownership could be to play a motivational role for selfprotection. The fact that response to threat to the rubber hand is correlated with the level of experienced ownership (González-Franco, Peck, Rodríguez-Fornells, \& Slater, 2014), already suggests involvement in self-defense and planning of protective movements. One of the sensorimotor representations of the body that we are able to experience is the protective body schema, and within this schema it is not necessarily only our biological body that we defend, but anything we experience as our body at that point in time. If a body part is incorporated into our body schema, we can experience it as our own, and the protective body schema presents a special case in which planning of defensive behavior is required. Anything that falls within our protective body schema is experienced as our own, and thus is worth defending. The results of the current experiment fall within this line of reasoning, as we observe an increase in proprioceptive drift when participants are presented with angry vocalizations. However, it is important to consider that we did not observe a difference between vocalizations of positive or negative valence. This seems to suggest that affective vocalizations have a general effect of expanding the boundaries of what we consider our body. Importantly, the methods used in the current experiment are not sensitive to subtle differences in the temporal dynamics of these effects. Whereas both positive and negative emotions motivate us to attend to our environment (e.g. Keil et al., 2007), and affect the sensorimotor system in similar ways (see e.g. for for work with emotional vocalizations; Banissy et al., 2010; Warren et al., 2006), a key difference might be timing. Whereas it might not be crucial to immediately respond when confronted with laughter, a scream demands our immediate attention. Given the set-up of the RHI paradigm, no conclusion can be drawn on any possible chronometric effects of emotion on body ownership. Future research is needed to dissect the observed effects; it could be of interest to include a measure of action readiness (e.g. muscle contraction as measured with EMG) to the RHI paradigm to specifically test the evolutionary purpose of body ownership.

One striking observation in the current study is that the effects of emotional vocalizations on proprioceptive drift were not only apparent in the synchronous, but also the asynchronous stroking condition. Typically, the asynchronous stroking condition is not supposed to induce the illusion of experiencing the rubber hand as your own, reflected in lower proprioceptive drifts and questionnaire scores compared to the synchronous stroking condition, both also evident in the current experiment. The second experiment tried to address the issue of possible trial-to-trial carry over effects by revealing the real hand of the participant after each trial. Despite this manipulation, there were still effects of sound condition apparent in both synchronous and asynchronous trials in experiment 2 . One possible explanation for this could be that 
in the current study a hyper-realistic silicone hand was used, and many participants reported experiencing some degree of illusion already before the start of the experiment, by just having their own hand hidden from view and the rubber hand placed in an anatomically plausible position. Indeed, it has been shown that not only hand shape but also texture creates a stronger illusion (Haans, IJsselsteijn, \& de Kort, 2008), and that a vision only condition can create proprioceptive drifts as strongly as synchronous stroking (Rohde, Di Luca, \& Ernst, 2011). If we are to assume the strength of the illusion was altered because being confronted with affective vocalizations placed the participants in a different state, in which for example a protective body schema is triggered, this might explain why the effects are seen in both synchronous and asynchronous trials. Critically though, main effects of stroking condition were always apparent, which shows that there was indeed a stronger illusion in synchronous than asynchronous trials.

The results of the current experiment show that perceiving emotional vocalizations have the potential to alter susceptibility to the RHI. Given that this illusion is a way of assessing malleability of body ownership, these results demonstrate that in certain contexts we might be more willing to integrate something as belonging to our body than in others. This study provides the first steps into seeing how emotions and body ownership might interact, but further research will be needed to examine the exact mechanism underlying their interaction. 


\section{References}

Armel, K. C., \& Ramachandran, V. S. (2003). Projecting sensations to external objects: evidence from skin conductance response. Proceedings of the Royal Society of London B: Biological Sciences, 270(1523), 1499-506. https://doi.org/10.1098/rspb.2003.2364

Banissy, M. J., Sauter, D. A., Ward, J., Warren, J. E., Walsh, V., \& Scott, S. K. (2010). Suppressing sensorimotor activity modulates the discrimination of auditory emotions but not speaker identity. The Journal of Neuroscience: The Official Journal of the Society for Neuroscience, 30(41), 13552-13557. https://doi.org/10.1523/JNEUROSCI.0786-10.2010

Belin, P., Fillion-Bilodeau, S., \& Gosselin, F. (2008). The Montreal Affective Voices: A validated set of nonverbal affect bursts for research on auditory affective processing. Behavior Research Methods, 40(2), 531-539. https://doi.org/10.3758/BRM.40.2.531

Borgomaneri, S., Vitale, F., Gazzola, V., \& Avenanti, A. (2015). Seeing fearful body language rapidly freezes the observer's motor cortex. Cortex, 65, 232-245. https://doi.org/10.1016/j.cortex.2015.01.014

Botvinick, M., \& Cohen, J. (1998). Rubber hands “feel” touch that eyes see. Nature, 391(February), 756. https://doi.org/10.1038/35784

Bradley, M. M., Miccoli, L., Escrig, M. A., \& Lang, P. J. (2008). The pupil as a measure of emotional arousal and autonomic activation. Psychophysiology, 45(4), 602-607. https://doi.org/10.1111/j.1469-8986.2008.00654.x

de Gelder, B., Snyder, J., Greve, D., Gerard, G., \& Hadjikhani, N. (2004). Fear fosters flight: a mechanism for fear contagion when perceiving emotion expressed by a whole body. Proceedings of the National Academy of Sciences of the United States of America, 101(47), 16701-6. https://doi.org/10.1073/pnas.0407042101

Engelen, T., de Graaf, T. A., Sack, A. T., \& de Gelder, B. (2015). A causal role for inferior parietal lobule in emotion body perception. Cortex, 73, 195-202. https://doi.org/10.1016/j.cortex.2015.08.013

Flögel, M., Kalveram, K. T., Christ, O., \& Vogt, J. (2015). Application of the rubber hand illusion paradigm: comparison between upper and lower limbs. Psychological Research, 80(2), 298-306. https://doi.org/10.1007/s00426-015-0650-4

González-Franco, M., Peck, T. C., Rodríguez-Fornells, A., \& Slater, M. (2014). A threat to a virtual hand elicits motor cortex activation. Experimental Brain Research, 232(3), 875-887. https://doi.org/10.1007/s00221-013-3800-1

Haans, A., IJsselsteijn, W. A., \& de Kort, Y. A. W. (2008). The effect of similarities in skin texture and hand shape on perceived ownership of a fake limb. Body Image, 5(4), 389-394. https://doi.org/10.1016/j.bodyim.2008.04.003

Hajcak, G., Molnar, C., George, M. S., Bolger, K., Koola, J., \& Nahas, Z. (2007). Emotion facilitates action: a transcranial magnetic stimulation study of motor cortex excitability during picture viewing. Psychophysiology, 44(1), 91-7. https://doi.org/10.1111/j.1469-8986.2006.00487.x

Huis In 't Veld, E. M. J., Van Boxtel, G. J. M., \& de Gelder, B. (2014). The Body Action Coding System I: muscle activations during the perception and expression of emotion. Social Neuroscience, 9(3), 249-64. https://doi.org/10.1080/17470919.2014.890668 
IJsselsteijn, W. A., de Kort, Y. A. W., \& Haans, A. (2006). Hand I See Before Me? The Rubber Hand Illusion in Reality, Virtual Reality, and Mixed Reality. Presence: Teleoperators and Virtual Environments, 15(4), 455-464. https://doi.org/10.1162/pres.15.4.455

Keil, A., Bradley, M. M., Junghöfer, M., Russmann, T., Lowenthal, W., \& Lang, P. J. (2007). Cross-modal attention capture by affective stimuli: evidence from event-related potentials. Cognitive, Affective \& Behavioral Neuroscience, 7(1), 18-24. https://doi.org/10.3758/CABN.7.1.18

Komeilipoor, N., Pizzolato, F., Daffertshofer, A., \& Cesari, P. (2013). Excitability of Motor Cortices as a Function of Emotional Sounds. PLoS ONE, 8(5), e63060. https://doi.org/10.1371/journal.pone.0063060

Morgan, H. L., Turner, D. C., Corlett, P. R., Absalom, A. R., Adapa, R., Arana, F. S., ... Fletcher, P. C. (2011). Exploring the impact of ketamine on the experience of illusory body ownership. Biological Psychiatry, 69(1), 35-41. https://doi.org/10.1016/j.biopsych.2010.07.032

Pavani, F., Spence, C., \& Driver, J. (2000). Visual Capture of Touch: Out-of-the-Body Experiences With Rubber Gloves. Psychological Science, 11(5), 353-359. https://doi.org/10.1111/1467-9280.00270

Pavani, F., \& Zampini, M. (2007). The role of hand size in the fake-hand illusion paradigm. Perception, 36(10), 1547-1554. https://doi.org/10.1068/p5853

Roelofs, K., Hagenaars, M. A., \& Stins, J. (2010). Facing freeze: social threat induces bodily freeze in humans. Psychological Science, 21(11), 1575-1581. https://doi.org/10.1177/0956797610384746

Rohde, M., Di Luca, M., \& Ernst, M. O. (2011). The Rubber Hand Illusion: Feeling of Ownership and Proprioceptive Drift Do Not Go Hand in Hand. PLoS ONE, 6(6), e21659. https://doi.org/10.1371/journal.pone.0021659

Schutter, D. J. L. G., Hofman, D., \& Van Honk, J. (2008). Fearful faces selectively increase corticospinal motor tract excitability: A transcranial magnetic stimulation study. Psychophysiology, 45(3), 345-348. https://doi.org/10.1111/j.1469-8986.2007.00635.x

Seth, A. K. (2013). Interoceptive inference, emotion, and the embodied self. Trends in Cognitive Sciences, 17(11), 565-573. https://doi.org/10.1016/j.tics.2013.09.007

Sforza, A., Bufalari, I., Haggard, P., \& Aglioti, S. M. (2010). My face in yours: Visuo-tactile facial stimulation influences sense of identity. Social Neuroscience, 5(2), 148-162. https://doi.org/10.1080/17470910903205503

Tieri, G., Tidoni, E., Pavone, E. F., \& Aglioti, S. M. (2015). Body visual discontinuity affects feeling of ownership and skin conductance responses. Scientific Reports, 5, 17139. https://doi.org/10.1038/srep17139

Tsakiris, M. (2010). My body in the brain: A neurocognitive model of body-ownership. Neuropsychologia, 48(3), 703-712. https://doi.org/10.1016/j.neuropsychologia.2009.09.034

Tsakiris, M., \& Haggard, P. (2005). The rubber hand illusion revisited: visuotactile integration and selfattribution. Journal of Experimental Psychology. Human Perception and Performance, 31(1), 80-91. https://doi.org/10.1037/0096-1523.31.1.80

Tsakiris, M., Tajadura-Jiménez, A., \& Costantini, M. (2011). Just a heartbeat away from one's body: interoceptive sensitivity predicts malleability of body-representations. Proceedings. Biological Sciences / The Royal Society, 278(1717), 2470-2476. https://doi.org/10.1098/rspb.2010.2547 
van Loon, A. M., van den Wildenberg, W. P. M., van Stegeren, A. H., Hajcak, G., \& Ridderinkhof, K. R. (2010). Emotional stimuli modulate readiness for action: a transcranial magnetic stimulation study. Cognitive, Affective \& Behavioral Neuroscience, 10(2), 174-81. https://doi.org/10.3758/CABN.10.2.174

Warren, J. E., Sauter, D. a., Eisner, F., Wiland, J., Dresner, M. a., Wise, R. J. S., ... Scott, S. K. (2006). Positive Emotions Preferentially Engage an Auditory-Motor "Mirror" System. Journal of Neuroscience, 26(50), 13067-13075. https://doi.org/10.1523/JNEUROSCI.3907-06.2006 



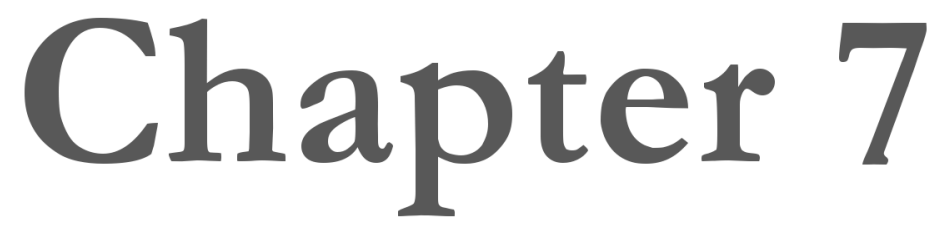

\section{Gestalt-like reappearance of visual afterimages induced by early visual cortex TMS}

Based on:

Engelen, T.*, Rademaker, R.L.* , \& Sack, A.T. (in preparation). Gestalt-like reappearance of visual afterimages induced by early visual cortex TMS. 



\begin{abstract}
When a positive afterimage is elicited at a moment when your hands are within your field of view, a conflict can be created between vision and proprioception. When one of the hands is moved outside the field of vision, the eyes will continue signaling one location of the hand, while proprioception signals another. In this instance, proprioception dominates over vision by causing a gestalt-like fading of the moved hand from the afterimage. In the current study we tested whether this fading of a removed hand from a positive afterimage is the result of targeted inhibitory feedback to early visual cortex (EVC), by examining how online stimulation of this area alters the amount of perceived fading. In the first experiment, participants removed either their left or right hand after a positive afterimage had developed, after which immediately TMS was applied (sham, single pulse, or triple pulse). Then, the relative strength of the percept of both hands was rated again. Results showed that, compared to sham and single pulse stimulation, triple pulse stimulation resulted in the ratings between both hands becoming more similar. However, this occurred both when the left and the right hand were removed, despite only right EVC (and thus the left hemifield) being targeted. Therefore a second experiment was conducted, in which the strength of the percept of both hands was rated separately. In addition, a condition was added in which neither of the hands was removed, which allowed testing if TMS altered the percept of the hands per se. Results again showed that triple pulse stimulation resulted in less visual fading over time of both the right and the left hand, while the condition in which neither hand was removed remained unaffected. Together, these results demonstrate that lifting EVC from inhibition can indeed reverse the effects of visual fading. In addition, feedback to EVC might originate from multimodal integration sites in which both hands are represented.
\end{abstract}

\title{
Introduction
}

If you've ever stared steadily enough at a paint chip on a wall, or a crack in the ceiling, you may have noticed the peripheral world slowly fading into oblivion. This visual fading, known as the Troxler effect (Troxler, 1804) is one of multiple examples of visual fading on record (Billock \& Tsou, 2004; Ditchburn \& Ginsborg, 1952; Riggs, Ratliff, Cornsweet, \& Cornsweet, 1953). It is generally understood that small transients in the visual input, such as those induced via microsaccades, are critical to establish a visual percept from light entering the eyes, while images that are stable on the retina will result in the visual world fading away (Martinez-Conde, Macknik, \& Hubel, 2004; Martinez-Conde, Otero-Millan, \& MacKnik, 2013). While the phenomenon of visual fading has a long history in experimental psychology (Darwin, 1795), its neural substrate remains poorly understood. One potential mechanism is that stable visual signals are actively inhibited at the level of Early Visual Cortex (EVC). As a direct test of this hypothesis, here we used Transcranial Magnetic Stimulation (TMS) over EVC while a retinally stable afterimage faded from view. We demonstrated that visual fading is not inevitable, and can be partly reversed by EVC stimulation.

It is widely recognized that visual percepts are shaped by more than solely the input originating from the retina. This is demonstrated by illusions revealing a mismatch between what people report seeing, and the information entering their eyes. For example, during perceptual fillingin, information is perceived that is not directly sensed by the eyes (Komatsu, 2006; Shimojo, Kamitani, \& Nishida, 2001). Similarly, perceptual fading is an example of an image sensed at the level of the retina, while that image is not being perceived (Billock \& Tsou, 2004; Martinez- 
Conde et al., 2004, 2013). The subjective percept, and not retinal inputs, seems to determine responses at the earliest levels of visual processing. In perceptual filling-in, fMRI activity in primary visual area V1 tracked the subjectively perceived filled-in surface between two moving gratings (Meng, Remus, \& Tong, 2005), and an illusory colored surface (Sasaki \& Watanabe, 2004), rather than the objectively presented blank gap. A similar demonstration in the context of afterimages comes from modulation of retinotopic activity in V1 by perceived size changes of an afterimage, despite constant retinal input (Sperandio, Chouinard, \& Goodale, 2012). Objects that appeared to occupy more space in the visual field also activated a greater portion of V1 (Murray, Boyaci, \& Kersten, 2006). Neural correlates of perceptual filling-in can extend beyond V1, and have been found in higher-level areas including parietal cortex (Mendola, Conner, Sharma, Bahekar, \& Lemieux, 2006). A putative causal link between parietal cortex and what people perceive visually, comes from one study showing that TMS over Inferior Parietal Sulcus (IPS) induced fading-like perceptual disappearance of peripherally presented stimuli (Kanai, Muggleton, \& Walsh, 2008).

Thus, conscious percepts during visual illusions, including perceptual fading, seem to involve changes at the earliest levels of sensory processing. Interestingly, it is common for perceptual fading to occur in meaningful chunks, following Gestalt-like principles (Billock \& Tsou, 2004). This implies the directed involvement of higher-level areas, able to process information at a more semantic level. Given the involvement of both higher- and lower level areas of the brain, what might the mechanisms underlying perceptual fading be? Because changes in early sensory cortex generally track perception, here we hypothesize that targeted top-down inputs to sensory cortex can impose the changes required for the perceptual experience of gestalt-like fading in afterimages. To test this hypothesis, we utilized positive afterimages to induce perceptual fading. Positive afterimages can be elicited by discharging a brief flash of light after a prolonged period of dark adaptation. In the total darkness after the flash, a grey-scale visual afterimage develops of everything that was perceived during the flash. The experience can be described as a dim light turning on, with lights and darks having the correct sign.

Positive afterimages are a relatively easy means to the retinal stabilization of a scene and, importantly, they lend themselves well for inducing a conflict between vision and proprioception (Davies, 1973). In everyday life, visual and proprioceptive modalities will generally be in congruence with one another, i.e. when reaching for an object you will both see your hand reaching towards the object, as well as receive proprioceptive information on the trajectory of your hand. When viewing a positive afterimage, a situation can be created during which these two modalities convey incongruent messages to the brain. For example, when an afterimage is elicited while one is viewing one's own hand, and subsequently that hand is moved out of the afterimage, the hand will appear to fade or crumble, while the rest of the afterimage stays intact (Davies, 1973; Gregory, Wallace, \& Campbell, 1959). In this case, proprioception has a catastrophic impact on visual perception when information from the two senses is in conflict (i.e. proprioception indicates the hand is removed, while the retinal input indicates the hand is still in view). Note that this resolution to the sensory conflict is somewhat unique, as in most cases a conflict between vision and proprioception is resolved in favor of the visual sense (Hay, Pick, \& Ikeda, 1965), presumably due to its high spatial acuity and reliable signals.

More subtle effects occur when a person moves their hand towards or away from them in a positive afterimage. Such movements induce a perceived change in size of the hand in a manner that follows Emmert's law, which states that a retinal image is proportional in size to the 
distance of the surface it is projected on (Edwards \& Boring, 1951). Practically speaking this means that the size of the hand will appear to grow or shrink, depending on the direction of the movement of the hand in the afterimage (i.e. away or towards the eyes, respectively; Carey \& Allan, 1996). Both fading and size scaling of a hand in an afterimage occur irrespective of whether the movement is active or passive, suggesting that afferent proprioceptive information is sufficient to influence the percept (Bross, 2000; Hogendoorn, Kammers, Carlson, \& Verstraten, 2009), and changes of the hand in the afterimage happen only when the viewed hand is perceived as one's own (Faivre et al., 2017).

In these examples of perception in positive afterimages, there is a clear impact on visual processing that comes either directly or indirectly from the sense of proprioception. A proposed mechanism for modulation of EVC activity through other modalities is via back-projections originating in multimodal integration areas, such as posterior parietal cortex (Macaluso et al., 2000; Bolognini \& Maravita, 2007). Anatomically, early visual areas are indeed innervated by connections originating in multimodal areas of the brain (Blatt, Andersen, \& Stoner, 1990; Cavada \& Goldman-Rakic, 1989; Lewis \& Van Essen, 2000). At the neuronal level, the integration of vision and proprioception in multimodal areas has been extensively studied in monkeys (Graziano, Cooke, \& Taylor, 2000; Sakata, Takaoka, Kawarasaki, \& Shibutani, 1973). Visually responsive neurons in ventral premotor cortex of macaque have been shown to continually update their visual receptive fields to track an effector as the effector moves through space (Graziano, Hu, \& Gross, 1997). Such findings suggest a locus for sensory integration, and a potential mechanism supporting the smooth everyday interactions with real-world environments through sensory-guided actions (Maravita, Spence, \& Driver, 2003). Recent neuroimaging work in humans has implicated similar loci of visual-proprioceptive integration (Limanowski \& Blankenburg, 2016). When participants saw a virtual arm at a location that was congruent with the position of their real arm, increased activity was observed in posterior parietal cortex, compared to when the virtual and real arm were at incongruent locations. Interestingly, when visual and proprioceptive information were congruent, correlations between integration areas, such as posterior parietal cortex, and primary visual cortex (V1) implied direct communication between these areas (Limanowski \& Blankenburg, 2016).

Importantly, multi-modal interactions have the capacity to alter EVC activity, as has been demonstrated by studies on cross-modal facilitation effects. For example, when tactile stimulation of the hand coincided with a visual stimulus on the same side of space, detection of the visual stimulus was facilitated, and visual cortex activity enhanced (Macaluso et al., 2000). Similarly, one study assessed visual cortex excitability by inducing visual phosphenes, which are briefly perceived flashes of light in response to a TMS pulse over EVC. A higher level of cortical excitability is assumed to lower the threshold for eliciting a phosphene. Visual cortex excitability was increased at locations of the visual field that coincided with the location at which a hand was touched (Ramos-Estebanez et al., 2007). This effect occurred both with the hands crossed and uncrossed, always showing increased excitability when phosphene location and touch were coincident, irrespective of the hand that was touched, which demonstrates remapping of visual tactile space (Bolognini \& Maravita, 2007).

In sum, there is ample evidence to suggest that top-down influences from multimodal integration areas can directly innervate and impact processing in EVC. The sensory conflict evoked when removing a hand from a positive afterimage, and the subsequent gestalt-like fading of the removed hand, could therefore result from targeted feedback from multimodal areas onto 
EVC. Specifically, fading of the hand could be due to the active inhibition of the bottom-up signals associated with viewing that hand. In fact, active inhibition might more broadly serve as the mechanism responsible for perceptual fading of stabilized images: In the absence of visual transients, information might be deemed irrelevant by the visual system, and hence systematically filtered out of the perceptual experience.

Here, we tested this hypothesis by combining fading in positive afterimages with online TMS over EVC. If perceived gestalt-like fading of a hand is indeed the result of targeted inhibition at the level of EVC, disrupting this inhibition by means of TMS should weaken the extent to which the hand is perceived to fade (i.e. lead to a temporary 'recovery' of the faded hand). In two separate experiments, participants viewed afterimages of both their hands held out in front of them. In Experiment 1, one of the hands was removed, and participants rated the relative clarity of their two hands immediately before, and immediately after, a TMS-manipulation over right EVC. Manipulations were a single TMS pulse, a triple TMS pulse, or a single auditory pulse (sham TMS) that sounded like a real TMS pulse. In Experiment 2 only triple pulse sham and real TMS were administered and participants rated both hands individually to disentangle the effects of TMS on the hands ipsilateral and contralateral to the targeted right hemisphere. In both experiments we demonstrated that the gestalt-like fading of the removed hand was systematically reduced after administering TMS. This implies that TMS can provide temporary relief from the factors responsible for perceptual fading, and suggests that an active top-down inhibitory mechanism impacting visual processing at the level of EVC could be at play during perceptual fading.

\section{Methods}

\section{Participants}

Participants in Experiment 1 were ten healthy volunteers recruited from Maastricht University with a mean age of 25.8 years $(\mathrm{SE}=3.3,8$ females). Ten more participants took part in Experiment 2, of whom two had previously participated in Experiment 1 (mean age 25.5 years, SD $=2.8 ; 6$ females). All participants were right handed, had normal or corrected-to-normal vision, and were naïve to the purpose of the experiment (except for author RR in Experiment 2). Before the start of the experimental proceedings, participants provided written informed consent and were screened for TMS safety based on published safety guidelines (Rossi, Hallett, Rossini, \& Pascual-Leone, 2012). In total, we excluded nine people from participation on the grounds that they were unable to perceive phosphenes $(\mathrm{N}=2)$, unable to perceive phosphenes at the desired visual field location $(\mathrm{N}=1)$, unable to experience an afterimage long enough to give the ratings within the time the image was perceived $(\mathrm{N}=2)$, or unable to experience fading in the afterimage in response to bodily movement (see also procedures, $\mathrm{N}=4$ ). This study was approved by the standing ethical committee of the Psychology and Neuroscience department at Maastricht University. Except for one of the authors, participants received monetary reimbursement for their time.

\section{Materials}

The experiments took place in a completely darkened room. Because some scattered light could not be avoided, a large cardboard box $(65 \times 74 \times 95 \mathrm{~cm})$ placed atop a table was used to enclose 
participant's field of view and shield them from stray light, thus ensuring absolute darkness (Figure 1). Head and TMS coil stability were ensured by a forehead- and chinrest, together with a coil-holder firmly fixing the TMS coil over the participant's skull. To allow stable fixation in the dark, dim red LED light was shone through a small hole in the box, creating a fixation of $<0.25^{\circ}$ of visual angle at a viewing distance of $55 \mathrm{~cm}$. The wavelength of red light $(620-750$ $\mathrm{nm}$ ) falls outside of the response range of retinal rods (Wald, 1955), and as such did not interfere with dark adaptation. To ensure stable arm and hand positions from one trial to the next, a horizontal bar was placed above the tabletop. This distance required participants to raise their elbows up from the tabletop to place their hands behind the bar. Participant's arms were obscured from view by means of a black plastic arm-occluder attached to the bar, and the lateral hand distance was fixed by two markers behind the bar that were readily discernable by touch. To elicit positive afterimages, a Vivitar 285HW Zoom Thyristor flashgun was placed in the box pointing at the white box-ceiling above the participant.

Biphasic TMS stimulation was applied with a MagPro R30 stimulator (Medtronic Functional Diagnostics A/S, Skovlunde, Denmark; maximum stimulator output 1.9T) and a figure-ofeight coil (MCB70). The coil was held throughout the experimental procedures by a custommade coil-holder, ensuring stable placement over the skull. Auditory sham pulses were delivered over an audio speaker attached to the TMS coil. Sham sounds were recorded with an iRiver recording device and edited to filter out background noise with the Audacity software package. Volume (in $\mathrm{dB}$ ) was measured with a decibel meter for both sham and real pulses across a range of intensities, and the volume of the sham pulses was equated to match that of the real TMS pulses. To control trial timing in the dark, auditory beeps indicated the start of every new trial at 40-second intervals. Beeps were played from a computer, and were generated using the Presentation software package (Experiment 1) and Matlab with the Psychophysics toolbox (Experiment 2; Brainard, 1997; Pelli, 1997).
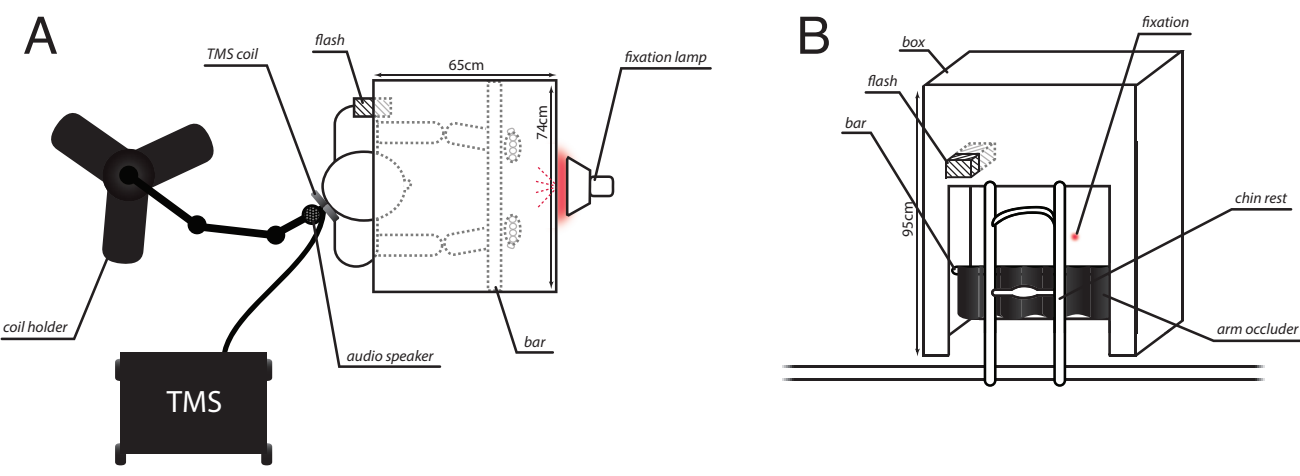

Figure 1. Experimental setup. (A) Top view of cardboard box with participant seated in the experimental setup. Stimulator and coil-holder are also depicted. (B) Front view of cardboard box atop of the table, depicting also the chinrest and fixation. Images are scaled to true size.

\section{Procedure}

Each of the two experiments consisted two separate sessions: A preliminary and an experimental session. The purpose of the preliminary session was (1) to check if participants could perceive phosphenes, (2) to familiarize participants with afterimages and experimental 
procedures, (3) to practice the rating scale, and (4) to screen participants, and see whether movement of a hand resulted in perceived fading of that hand from the afterimage. This screening procedure was adapted from previous work (Carlson, Alvarez, Wu, \& Verstraten, 2010; Rademaker, Wu, Bloem, \& Sack, 2014) and required because both Experiments 1 and 2 critically depended on participant's ability to perceive fading in response to bodily movements.

During the preliminary session participants were seated with their head in the chinrest, and instructed to fixate throughout. This session consisted of 15 practice trials performed after 10 minutes of dark adaptation: The first 3 trials were to familiarize participants with afterimages, without performing a task. During the following 6 trials, participants placed their hands behind the horizontal bar with their palms facing towards them. After the flash, the left or right hand was removed (or both were kept stationary in experiment 2), and participants described their percept. During the final 6 trials participants practiced quantifying their subjective fading experiences, using the rating scale. For Experiment 1 we used a rating scale that relied on a relative judgment of the two hands on a 11-point scale (adapted from Hogendoorn, Kammers, Carlson, \& Verstraten, 2009), and for Experiment 2 we used a variant of this scale that allowed the hands to be judged independently on a 7 -point scale (Figure 2C and Figure 2D respectively). The preliminary session took approximately 1 hour to complete. Some participants were allowed extra practice trials in case of difficulties mastering the task and/ or rating scale.
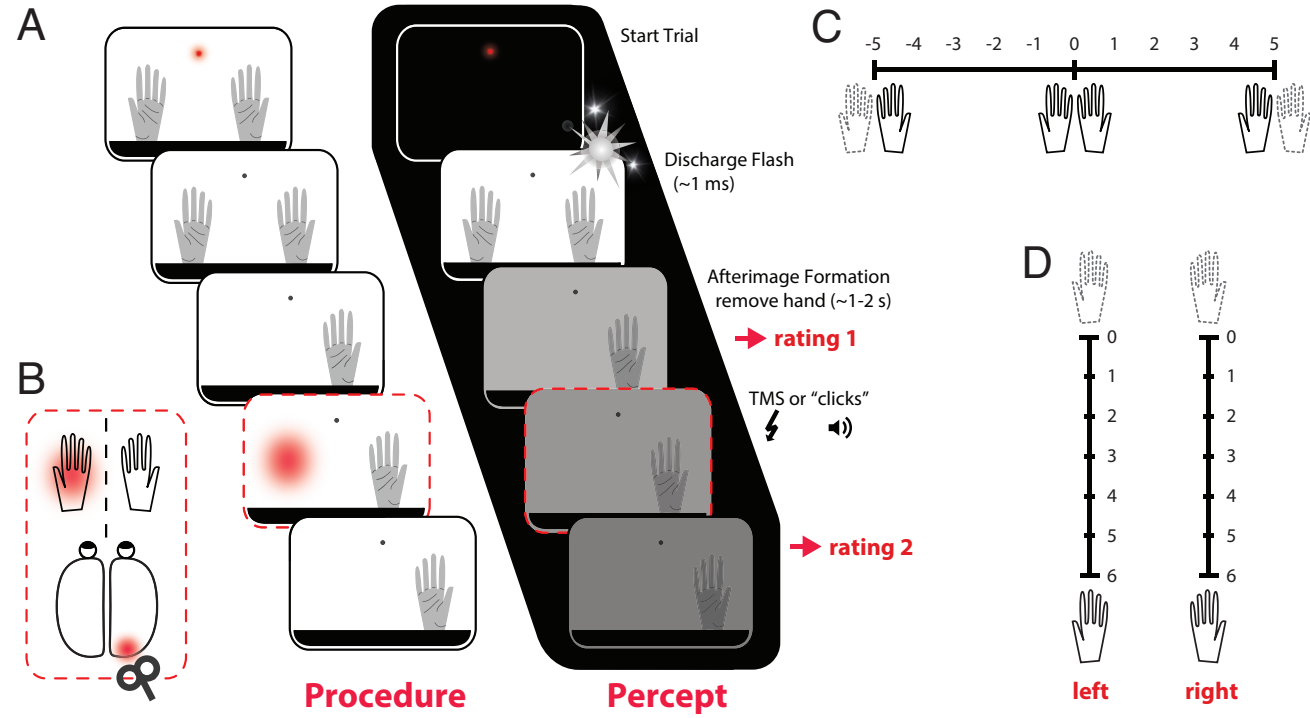

Figure 2. Trial sequence, stimulation, and rating scales. (A) At the start of each trial, participants put their hands up behind the black bar and started fixation aided by a small red light. When participants indicated that they were ready to start the trial, a brief flash was emitted, and once the afterimage had developed participants removed one of their hands (or kept both stationary). After briefly observing their hands, participants gave a rating, which triggered sham (auditory clicks) or TMS pulses. After the pulses, participants again briefly observed their hands and provided a second rating. The next trial started 40 seconds after the start of the previous trial, and in between trials participants could rest their arms, and move their eyes around freely. (B) TMS was applied over the right part of Early Visual Cortex corresponding to the left part of visual space. To stimulate the part of the visual field containing the left hand, prior to the start of the experiment phosphenes were elicited and the coil moved around until the left hand and phosphenes overlapped. During the experiment itself, stimulation was applied at $80 \%$ of phosphene threshold to 
ensure participants would not perceive any phosphenes. (C) Rating scale Experiment 1. A rating of zero would indicate that both hands were perceived with equal strength. A rating of -5 indicated that the left hand had faded entirely, while the right hand was perceived as veridical. A rating of 5 meant the opposite, with the left hand being perceived as veridical, and the right hand having faded completely. (D) Rating scale Experiment 2. Half of the participants would always rate first the left, then the right hand, while the other half of participants always rated first the right, then the left hand.

An experimental session started by positioning the coil over the right posterior part of the skull, and identifying the part of early visual cortex corresponding to the visual field position of the left hand by means of phosphene localization (Figure 2B). Once properly localized, the coil was fixed over the skull with a coil-holder, and participant's phosphene thresholds were determined in an already dimmed room, using a brief staircase procedure. Subsequent stimulation was applied at $80 \%$ of phosphene threshold (mean $=31.7 \%$ of stimulator output with $\mathrm{SD}=4.8 \%$ in experiment 1 , and mean $=39.2 \%$ of stimulator output with $\mathrm{SD}=10.9 \%$ in experiment 2). After ten minutes of dark adaptation, participants completed 6 blocks of 15 trials per block. A trial (Figure 2A) started by participants placing their hands behind the bar, and after the flash was emitted and an afterimage had formed, participants removed one of their hands (or kept both up) and after a brief period of observation gave a rating. This first rating triggered the TMS or sham pulses (Figure 2A), after which participants again observed and rated their hands. Participants fixated throughout each trial. To avoid drift of the afterimage relative to fixation, the red light was always switched off simultaneously with the flash, after which participants continued to fixate the unlit fixation spot. Participants could rest their arms on the tabletop in between trials.

The six experimental blocks of Experiment 1 consisted of two conditions during which a hand was removed (either the left or right hand), and three stimulation conditions (either single pulse sham, single pulse TMS, or triple pulse TMS at 10Hz). Because TMS targeted the left part of visual space overlapping with the position of the left hand, this design allowed us to evaluate the effect of TMS on fading when the left hand was removed. Moreover, this could be compared to how TMS affected fading of the right hand, and to fading during sham instead of TMS. Experiment 2 consisted of three conditions involving the hands (left hand was removed, right hand was removed, or both hands remained stationary) and two stimulation conditions (triple pulse sham or triple pulse TMS at $10 \mathrm{~Hz}$ ). The stationary condition allowed us to see how TMS affected the percept of a hand when there was only gradual fading of the entire afterimage occurring naturally over time. The order of blocks was counterbalanced between participants. An experimental session lasted between 1.5 and 2 hours.

\section{Results}

\section{Experiment 1}

Participants gave two ratings on a relative scale (Figure 2C) - one right before, and one right after the pulse. If the left hand were less visible than the right, participants would provide a negative rating. Conversely, positive ratings would be given if the right hand were faded relative to the left. If both hands were equally visible, the rating would be " 0 ".

To verify that participants experienced gestalt-like fading of the hand removed from the afterimage before the pulse (Figure 3A; green bars), we performed a 2 (hand) x 3 (TMS condition) repeated-measures ANOVA against zero. Note that to equate the effects of removing the left and right hands, we inverted the sign of the ratings given when the left hand was removed 
before statistical testing. Thus, one can consider the negative-going average ratings displayed in the top panel of Figure 3A as positive for the purposes of interpreting all subsequently analyses reported here. Removing a hand from the afterimage resulted in perceived fading of that hand $\left(F_{(1,9)}=93.150, p<.001, \eta_{\mathrm{p}}^{2}=0.912\right)$, with average (positive) ratings between 1.97 and 2.42 across conditions. Moreover, equal amounts of fading were experienced before the pulse, with no differences between the left and right hands $\left(F_{(1,9)}=1.390, p=0.269, \eta^{2}{ }_{\mathrm{p}}=\right.$ $0.134)$, nor any differences between TMS conditions $\left(F_{(2,18)}=0.118, p=0.889, \eta_{\mathrm{p}}^{2}=0.013\right)$. Indeed, no differences were expected at this baseline rating, provided prior to the application of TMS.

A

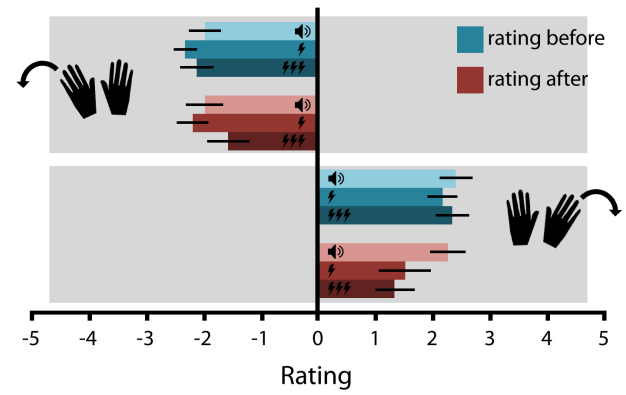

B

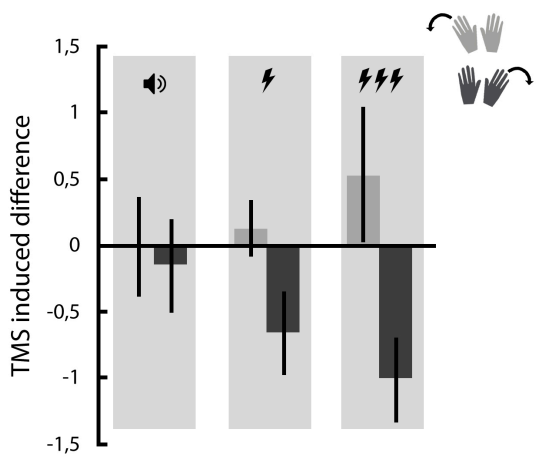

Figure 3. The effects of sham and TMS on fading of the left and right hands in Experiment 1. (A) Participants rate the relative visibility of their hand after removing one hand from view both before and after a pulse (sham, single pulse, or triple pulse TMS). Before the pulse (green bars) there were no differences between pulse conditions. After the pulse there was a difference between the pulse conditions, with relative ratings closer to zero after triple pulse compared to sham TMS. This indicates that after triple pulse TMS the two hands were rated as more similar in their degree of visibility. (B) Data in (A) are replotted to more clearly show the impact of the pulse by contrasting the ratings before and after the pulse. A difference of zero indicates no change in relative ratings before and after the pulse. For trials on which the left hand was removed (light grey) a positive score indicates that the pulse made the two hands appear more similar in terms of their visibility. Similarly, for trials on which the right hand was removed (dark grey) a negative score indicates that the pulse made the two hands appear more similar in terms of their visibility.

Next, we investigated the impact of the pulse on perceived fading (compare green and red bars in Figure 3A). A 2 (hand) x 3 (TMS condition) x 2 (before and after rating) repeated measures ANOVA showed a significant interaction between TMS condition and before/after rating $\left(F_{(2,18)}=3.940, p=0.038, \eta_{\mathrm{p}}^{2}=.304\right)$. We followed up on this interaction by first collapsing the data across the hand condition, as relative ratings were no different for the left and right hand $\left(F_{(1,9)}<0.001, p=0.997, \eta_{\mathrm{p}}^{2}<0.001\right)$. To compare the before and after ratings, we performed 3 post-hoc t-tests, each within a given TMS condition. Perceived fading did not change between the first (before) and second (after) rating in the sham condition (mean before $(\mathrm{SE})=$ $2.20(0.26)$, mean after $(\mathrm{SE})=2.13(0.28) ; t(10)=0.214, p=0.835)$, nor was there a significant change with single pulse TMS (mean before $(\mathrm{SE})=2.25(0.19)$, mean after $(\mathrm{SE})=1.86(0.37)$; $t(10)=1.786, p=0.108)$. When triple pulse TMS was applied, ratings after the pulses were marginally lower than before TMS (mean before $(\mathrm{SE})=2.23(0.26)$, mean after $(\mathrm{SE})=1.46(0.28)$; $t(10)=2.197, p=0.056$ ). This indicates that the relative visibility of the two hands became more similar after triple pulse TMS, irrespective of whether the left or right hand was removed. To compare TMS conditions, we performed two repeated-measures ANOVA's, one within 
the before, and one within the after ratings. As already reported above, there were no differences between TMS conditions at the first (before) rating $\left(F_{(2,18)}=0.118, p=0.889, \eta_{\mathrm{p}}^{2}=\right.$ 0.013). However, at the second (after) rating there was a significant main effect of TMS condition $\left(F_{(2,18)}=6.221, p=.009, \eta_{\mathrm{p}}^{2}=.409\right)$, driven by the difference between sham and triple pulse TMS $(t(9)=3.02, p=.043$, Bonferroni corrected).

Figure 3B replots the data in Figure 3A by showing the difference between the before and after ratings. Here, the further the difference score is away from zero, the more similar the hands became after the pulse (in positive and negative directions for removing the left and right hands, respectively). An analysis of the difference scores in a 2 (hand) x 3 (TMS condition) repeated-measures ANOVA revealed a significant main effect of pulse condition $\left(F_{(2,18)}=\right.$ $\left.3.960, p=0.038, \eta_{\mathrm{p}}^{2}=0.306\right)$, but no effect of hand $\left(F_{(1,9)}=1.338, p=0.277, \eta_{\mathrm{p}}^{2}=.129\right)$, and no interaction $\left(F_{(2,18)}=0.827, p=0.453, \eta_{\mathrm{p}}^{2}=0.084\right)$. The main effect of TMS condition was driven by a trending difference between sham and triple pulse TMS $(t(9)=2.816, p=0.06$, Bonferroni corrected), indicating the two hands were perceived as more similar after a triple TMS pulse, compared to sham. Together, these results suggest that triple-pulse TMS significantly reduced the amount of fading participants experienced after removing their hand from an afterimage, irrespective of the hand (the left or right) that was removed.

Experiment 2

The relative rating scale employed in Experiment 1 comes with two important limitations. First, under the assumption that TMS over right EVC exclusively targets the location of visual space encompassing the left hand, the relative rating should reflect perceived changes at this location. Thus, the TMS induced reduction in relative rating when the left hand was removed implies that TMS reinstated the visual representation of the left hand. Following the same logic, the TMS induced reduction in the relative rating when the right hand was removed implies that TMS reduced the quality of the percept of the stationary left hand, making it more similar in quality to the faded right hand. However, we cannot exclude alternative explanations for the results in Experiment 1. For example, if TMS over right EVC were able to also affect perception of the right hand, relative ratings would not dissociate between what is happening at the two hands. To illustrate this point, consider a trial during which the left hand was removed: If the relative rating was closer to zero after applying TMS, this could mean that the perceived quality of the left hand was improved, or alternatively, that the quality of the right hand was reduced. Both would make the hands appear qualitatively more similar. A second limitation of the relative rating scale is that it's not sensitive to detecting overall fading of hands in the afterimage: Two hands that were both very crisp would receive a rating of zero, while two hands that had nearly faded to an equal extent would also get a rating of zero.

To address these limitations, Experiment 2 required participants to rate the left and right hand independently, and on an absolute scale from 0-6 (where 0 indicates complete fading of the hand, and 6 the crispest possible afterimage of the hand). This allowed us to directly observe the effect of TMS on each individual hand, and also to track the overall levels of fading of the hands in the afterimage. Furthermore, Experiment 2 included a condition in which neither hand was removed, allowing us to observe potential effects of TMS on an afterimage in the absence of gestalt-like fading. Only triple pulse sham and real triple pulse TMS were used in this experiment. 
We first analyzed the data as we did in Experiment 1 to verify that our effects replicated. To this end, a relative rating was calculated by subtracting the ratings of the individual hands (left minus right; Figure 4). As before, we inverted the sign of the left hand relative ratings before statistical testing to equate the direction of the effect between the two hands. We confirmed that removing both the left $\left(F_{(1,9)}=13.740, p=0.005, \eta_{\mathrm{p}}^{2}=0.604\right)$ and right $\left(F_{(1,9)}=20.896, p\right.$ $\left.=0.001, \eta_{\mathrm{p}}^{2}=0.699\right)$ hand from the afterimage resulted in gestalt-like fading before the pulses were applied (Figure 4a, green bars; repeated-measures ANOVA's against zero), and the amount of fading was comparable for the left and right hand being removed $\left(F_{(1,9)}=0.510, p\right.$ $\left.=.493, \eta_{\mathrm{p}}^{2}=.054\right)$. When both hands were kept up, the right hand was relatively less visible than the left before the pulse $\left(F_{(1,9)}=7.781, p=0.021, \eta_{\mathrm{p}}^{2}=0.464\right)$, as indicated by the positivegoing relative difference. However, the relative ratings were only slightly above zero $(0.17$ and 0.22 for sham and real TMS, respectively) and these results did not hold up in direct t-tests against zero $(t(9)=1.98, p=.079$ and $t(9)=1.75, p=0.11$ for sham and real TMS, respectively).

We again analyzed the differences between before and after ratings (Figure 4B). A 2 (TMS condition) $\mathrm{x} 3$ (hand condition) repeated-measures ANOVA showed a main effect of the hand condition $\left(F_{(2,18)}=5.791, p=0.011, \eta_{\mathrm{p}}^{2}=0.392\right)$, driven by a marginal difference between the both hands up and remove right conditions $(t(9)=2.845, p=0.058)$, but not by any differences between the remove left and remove right conditions (stats). Importantly, ratings of the two hands became more similar after real TMS than after sham TMS $\left(F_{(1,9)}=8.077, p=0.019, \eta_{\mathrm{p}}^{2}\right.$ $=.473)$, as indexed by a larger deviation away from zero on the y-axis for real TMS, which indicates that the two hands went from relatively different at rating 1 , to relatively similar at rating 2 . There was no significant interaction $\left(F_{(2,18)}=3.164, p=0.066, \eta_{\mathrm{p}}^{2}=.260\right)$. These results replicate the findings of experiment 1 , insofar that real TMS caused relative differences to decrease compared to sham.

\section{A}

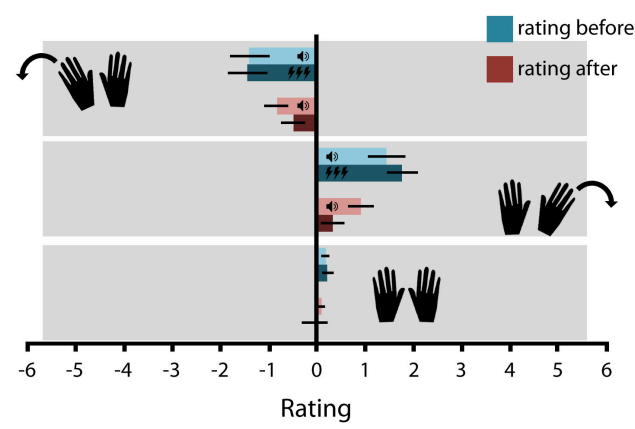

B

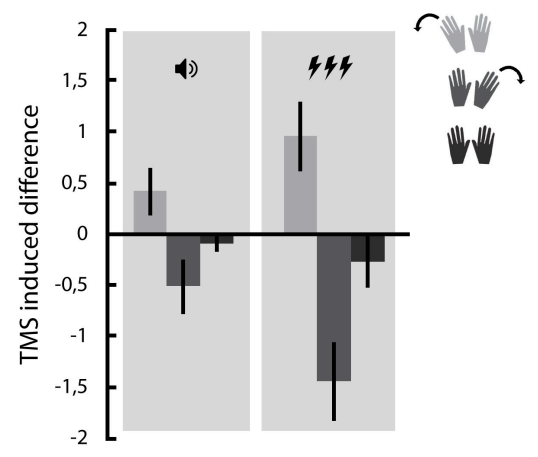

Figure 4. Experiment 2 and the impact of sound or TMS pulses on fading of the left and right hand. (A) Note that the scaling in Experiment 2 is slightly different from that in Experiment 1 (here from 0-6) This was done to ensure both Experiments had uneven rating scales when a rating of zero was included (11-point in Experiment 1, and 7-point in Experiment 2).

Next, we analyzed the data from Experiment 2 by capitalizing on the absolute rating scale. Beyond what can be gleaned from relative difference scores, absolute ratings allowed us to assess fading at each hand individually. We first calculated the difference between the beforeand after-ratings (Figure 5). A difference score of zero indicated no TMS induced change in 
the perceived quality of the hand, while negative going scores indicated an increase in the amount of fading after TMS compared to before.

For trials on which one of the hands was removed, we organized these difference scores according to what happened at the rated left or right hand. Specifically, we grouped difference scores by the rated hand being removed (i.e. right hand ratings when the right hand was removed, and left hand ratings when the left hand was removed) or stationary (i.e. right hand ratings when the left hand was removed, and left hand ratings when the right hand was removed). On trials where both hands were kept up, ratings of the left and right hand both refer to a situation in which a stationary hand was rated.

First of all, the results of a 3 (hand condition) x 2 (hand rated) x 2 (TMS condition) repeatedmeasures ANOVA showed no main effect of the hand being rated $\left(F_{(1,9)}=2.013, p=0.190\right.$, $\left.\eta_{\mathrm{p}}^{2}=0.183\right)$, suggesting that any changes in response to the experimental manipulation occurred similarly in both the left and the right hand. Importantly, there was a significant interaction between hand condition and TMS condition $\left(F_{(1,9)}=11.291, p=0.001, \eta_{\mathrm{p}}^{2}=0.556\right)$. To explore this interaction, we first collapsed the data across the hand being rated (left or right). Next, we wanted to assess fading at the removed and stationary hands and, critically, how TMS condition influenced fading in the different hand conditions.

To assess fading when a hand was either removed or stationary, we ran two 3-way repeated measures ANOVA, one for each TMS condition. Both ANOVAs revealed differences in fading between the three hand conditions (sham TMS: $F_{(2,18)}=4.755, p=0.022, \eta_{\mathrm{p}}^{2}=0.346$; and real TMS: $\left.F_{(2,18)}=12.186, p<0.001, \eta_{\mathrm{p}}^{2}=0.575\right)$. However, for sham TMS none of the posthoc pairwise comparisons remained significant (all $p>0.120$ ), indicating that the differences in fading were not robust between the three hand conditions. In contrast, post-hoc tests for real TMS showed significant differences between ratings of the removed hand and the stationary hand, both when the stationary hand was the only hand up $(t(9)=3.566, p=0.018$, Bonferroni corrected), as well as when both hands were up $(t(9)=3.566, p=0.018$, Bonferroni corrected).

Next we aimed to assess how TMS condition influenced fading within the separate hand conditions, which was tested with three 2-way ANOVAs, one for each hand condition. When the hand was removed, sham and real TMS had a different impact on perceived fading $\left(F_{(1,9)}=\right.$ $\left.5.422, p=0.045, \eta_{\mathrm{p}}^{2}=0.376\right)$, with smaller scores for real (mean $\left.(\mathrm{SE})=-0.33(0.24)\right)$ compared to sham TMS (mean (SE) $=-0.76(0.29)$ ). Because negative scores indicate that the hand fades from the first (before) to the second (after) rating, the smaller score for real TMS (and its proximity to zero) suggests that real TMS inoculated the removed hand from fading. When the hand was stationary, real TMS caused marginally more fading compared to sham TMS, both when the stationary hand was the only hand up $\left(F_{(1,9)}=4.702, p=0.058, \eta_{\mathrm{p}}^{2}=0.343\right)$, and when both hands were up $\left(F_{(1,9)}=4.052, p=0.075, \eta_{\mathrm{p}}^{2}=0.310\right)$. Scores were more strongly negative going with real TMS (mean $(\mathrm{SE})=-1.53(0.28)$ and mean $(\mathrm{SE})=-1.49(0.30)$ for one and both hands up, respectively) than with sham TMS (mean(SE) $=-1.29(0.29)$, mean(SE) = 1.26(0.29) for one and both hands up, respectively). 
A
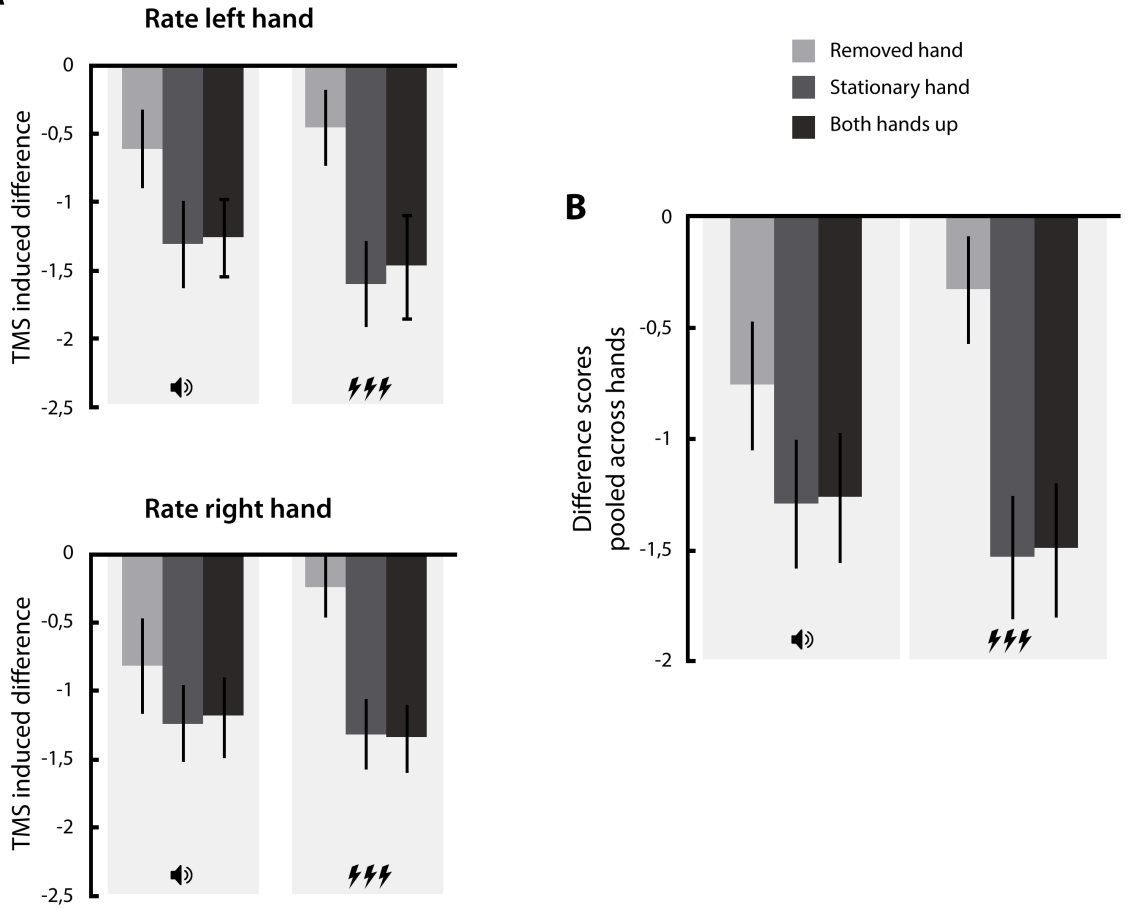

Figure 5. The effects of sham and TMS on fading of the left and right hands in Experiment 2. (A) Difference scores (rating after - rating before) are plotted against what occurred at the rated hand (i.e. 3 hand conditions). Hand condition was organized in three event types (1) the rated hand was removed, (2) the rated hand was stationary and the only hand kept up, and (3) the rated hand was stationary while both hands were kept up. More negative going scores indicate that the rated hand became less visible over time (i.e. between the first and second rating). (B) Replots the data in (A) pooled across the left and right hand.

\section{Discussion}

The current study set out to explore the mechanisms behind visual fading, and whether targeted inhibition of early visual cortex is at the core of this phenomenon. Specifically, we set out to explore whether online stimulation of early visual cortex would influence the gestalt-like fading of a hand that has been removed from a positive afterimage. By providing one way of retinally stabilizing images, positive afterimages are a valuable research tool into the origins of visual fading. When you are placed in complete darkness with your hands held within your field of vision while a brief flash occurs, a percept of your hands will develop. Once such an afterimage has fully developed, a conflict between vision and proprioception can be created by moving one of the hands from its initial position. After this relocation, the eyes will signal that the hand is still in its original location, whereas proprioceptive feedback will signal the new location of the hand. Interestingly, in this particular instance proprioception will dominate over vision, and the percept of the removed hand will fade from the afterimage in a Gestalt-like manner. One potential cause of such fading could be targeted inhibitory feedback to the early 
visual cortex $(\mathrm{EVC})$ originating from areas that are involved in multisensory integration, thereby 'forcing' the moved hand to be faded from the percept.

In experiment 1, participants were asked to remove either their left or right hand from their visual field, after an afterimage of both hands had developed. Once they reported that the removed had faded from the afterimage and had rated the relative intensity of the percept of the hands, either single pulse, triple pulse or sham TMS was applied over their right EVC (corresponding to the phosphene location of their left hand), after which they again rated the relative intensity of the percept of their hands. Results showed that after tpTMS, reports of the hands become more similar, which was contrary to the ratings given after either sham or spTMS. However, this result did not seem to be specific to the left hand, despite stimulation targeting right EVC, and additionally was locked to the phosphene location overlapping with the left hand. As the ratings used in experiment 1 were relative between the two hands, it was not possible to conclude that these results were caused by a reappearance of the right hand, or if stimulation altered the percept of the stationary left hand (when the right hand was removed). Therefore, we conducted a second experiment, in which a condition was included in which both hands were kept up, as well as having participants rate the intensity of the percept of their left and right hands separately. Once again, results showed that application of tpTMS, and not sham, resulted in the rating of the hands becoming more similar. We furthermore confirmed that this occurred because of reappearance of the left, as well as the right hand as a result of tpTMS. These results suggest that fading of hands in positive afterimages might indeed be driven by inhibitory feedback to EVC, however this feedback does not seem to be hemifield specific.

It has been established that activity in EVC is determined by what is subjectively perceived, rather than being a direct reflection of retinal input. For example, Sperandio et al. (2012) showed that activity in V1 reflects the perceived size of an afterimage, despite the size of the image on the retina remaining constant. Such findings suggest that bottom-up information gets muted by top-down inhibition, an idea which is further supported by the fact that visual fading occurs in gestalt-like chunks (Billock \& Tsou, 2004). As for the origin of such fading, posterior parietal cortex (PPC) is a likely candidate. Kanai et al. (2008) demonstrated that visual fading of a peripheral target can be elicited by stimulation of the intraparietal sulcus (IPS). The authors hypothesized that perceptual fading could be the result of degeneration of feedback originating in attention-related areas, such IPS.

In our study there is another important role of PPC to acknowledge, however, which is its role in multimodal integration. The fading of the hand from the positive afterimage is the result of a conflict between vision and proprioception, so one source of feedback could result from an area which integrates both sources. In humans, not many studies have specifically looked into how (and where) visual and proprioceptive information of the hands is integrated. Virtual reality is one method that allows for the creation of conflict between vision and proprioception and lends itself well for the combination with neuroimaging. One experiment combined fMRI acquisition with the presentation of photorealistic virtual hands that were either congruent or incongruent with the position of the real hand of the participant (Limanowski \& Blankenburg, 2016). In line with monkey research, they found that congruent visuo-proprioceptive presentations of the hand activated PPC and ventral premotor cortex, as well as the extrastriate body area. Additionally, psycho-physiological interactions analysis showed increased coupling between these integration areas and primary visual cortex when the presented information was 
congruent. The only observed activations for a contrast between incongruent versus congruent information was found in the anterior cingulate cortex. Another fMRI study used brief presentations of virtual hands that were either congruent or incongruent with the location of the real hand, but this time also hand laterality (with presentations of both the left and the right hand in their respective hemifields) was taken into account (Limanowski \& Blankenburg, 2017). Interestingly, a cluster in left inferior parietal lobule (IPL) responded preferentially to congruent visuo-proprioceptive hand information, but did so for both the right and the left hand (presented also in the left and right hemifield).

In the current study, we found that stimulation of right EVC affected the fading of both the left and the right hand (i.e. fading in both the left and right hemifield). The objective of the experiment was to target specifically the part of EVC corresponding to the location of the left hand in space. However, it cannot be excluded that stimulation (and in this case particularly the tpTMS stimulation) spread into the contralateral hemisphere through cortico-cortical connections, resulting in effects observed in both hemifields. Such spreading is not unlikely, as one high resolution EEG study demonstrated that application of a single TMS pulse to the occipital cortex can spread to the contralateral occipital cortex within $28 \mathrm{~ms}$ after stimulation (Ilmoniemi et al., 1997). Given that we applied multiple pulses, and in addition studied an effect that evolves in a margin of seconds, rather than milliseconds, it is possible that the observed bilateral effect is the result of exactly such spreading.

Another explanation of the effect observed in both hemifield might be found by considering the reference frame in which EVC receives feedback from multisensory integration areas. Previous work on multisensory integration has demonstrated that remapping of multisensory inputs occurs, binding them into a common reference frame that relates to the body. For example, visual cortex excitability (as measured in sensitivity for phosphene elicitation) is increased when touch co-occurs on the hand at phosphene location (Bolognini \& Maravita, 2007). This effect is hemifield dependent in that it occurs irrespective of whether the left or the right hand overlaps with phosphene location (i.e. conditions in which the hands are crossed or uncrossed). This suggests that multisensory feedback to EVC originates from a reference frame that incorporates information from both hemispheres, as EVC excitability can be altered by proprioceptive information originating from both contralateral and ipsilateral hand representations. The same experiment by Bolognini \& Maravita (2007) demonstrated that when PPC was stimulated with $1 \mathrm{~Hz}$ rTMS, this remapping was eliminated. While the crossmodal facilitation of EVC still occurred at the coincident location when the hands were uncrossed, when the hands were crossed it was the noncoincident location that led to increased EVC excitability (i.e. when the stimulus corresponded to the ipsilateral hemisphere). These results demonstrate a failure of PPC to effectively remap the occurrences into a new body-centered reference frame. This not only suggests that PPC is the locus of visuo-proprioceptive integration, but also that feedback to EVC originates from this area.

Enhanced excitability of EVC through crossmodal facilitation has also been demonstrated in neuroimaging studies that show enhanced visual cortex activity when vision and touch coincide (Macaluso et al., 2000). In addition, these studies again demonstrate that this enhanced EVC activity is the result of back-projections originating in parietal multisensory integration areas. This modulation of EVC is linked to a remapping to a common reference frame of the different primary senses, namely; when gaze was shifted with respect to the touched hand, the enhancement of EVC switched from one hemisphere to the other. This was even apparent if the 
hand was not visible to the participant, suggesting a role of proprioception (Macaluso, Frith, $\&$ Driver, 2002). When considering the current experiment, it thus seems that if integrated proprioceptive and visual feedback is send back to EVC, this occurs most likely within a new body-centered reference frame. However, this would most likely still happen in a hemifield specific manner, and thus does not explain the result that reappearance of the hand occurs in both hemifields, despite the stimulation of only one hemisphere. Nonetheless, as mentioned previously, left IPL was found to be responsive to both the left and right hemifield when integrating visuo-proprioceptive information (Limanowski \& Blankenburg, 2017). Further studies are necessary to try and uncover the origin of feedback projections to EVC, and how in these areas visuo-proprioceptive information is integrated. 


\section{References}

Billock, V. A., \& Tsou, B. H. (2004). What do catastrophic visual binding failures look like? Trends in Neurosciences, 27(2), 84-89. https://doi.org/10.1016/j.tins.2003.12.003

Blatt, G. J., Andersen, R. A., \& Stoner, G. R. (1990). Visual receptive field organization and corticocortical connections of the lateral intraparietal area (area LIP) in the macaque. Journal of Comparative Neurology, 299(4), 421-445.

Bolognini, N., \& Maravita, A. (2007). Proprioceptive Alignment of Visual and Somatosensory Maps in the Posterior Parietal Cortex. Current Biology, 17(21), 1890-1895. https://doi.org/10.1016/j.cub.2007.09.057

Carey, D. P., \& Allan, K. (1996). A motor signal and visual size perception. Experimental Brain Research, 110(3), 482-486. https://doi.org/10.1007/BF00229148

Carlson, T. A., Alvarez, G., Wu, D., \& Verstraten, F. A. J. (2010). Rapid Assimilation of External Objects Into the Body Schema. Psychological Science, 21(7), 1000-1005. https://doi.org/10.1177/0956797610371962

Cavada, C., \& Goldman-Rakic, P. S. (1989). Posterior parietal cortex in rhesus monkey: I. Parcellation of areas based on distinctive limbic and sensory corticocortical connections. Journal of Comparative Neurology, 287(4), 393-421.

Darwin, E. (1795). Zoonomia; or the laws of organic life (4th Americ). Philadelphia, PA: Edward Earle.

Davies, P. (1973). Effects of movements upon the appearance and duration of a prolonged visual afterimage: 1 . Changes arising from the movement of a portion of the body incorporated in the afterimaged scene. Perception, 2(2), 147-153.

Ditchburn, R. W., \& Ginsborg, B. L. (1952). Vision with a stabilized retinal image. Nature, 170(4314), 3637.

Edwards, W., \& Boring, E. (1951). What Is Emmert's Law? The American Journal of Psychology, 64(3), 416422.

Faivre, N., Dönz, J., Scandola, M., Dhanis, H., Bello Ruiz, J., Bernasconi, F., ... Blanke, O. (2017). SelfGrounded Vision: Hand Ownership Modulates Visual Location through Cortical $\beta$ and $\gamma$ Oscillations. The Journal of Neuroscience, 37(1), 11-22. https://doi.org/10.1523/JNEUROSCI.056316.2017

Graziano, M. S. A., Cooke, D. F., \& Taylor, C. S. R. (2000). Coding the Location of the Arm by Sight. Science, 290(5497), 1782-1786. https://doi.org/10.1126/science.290.5497.1782

Graziano, M. S., Hu, X. T., \& Gross, C. G. (1997). Visuospatial properties of ventral premotor cortex. Journal of Neurophysiology, 77(5), 2268-2292. https://doi.org/10.1152/jn.1997.77.5.2268

Gregory, R. L., Wallace, J. G., \& Campbell, F. W. (1959). Changes in the size and shape of visual afterimages observed in complete darkness during changes of position in space. Quarterly Journal of Experimental Psychology, 11(January 2015), 54-55. https://doi.org/10.1080/17470215908416288

Hay, J. C., Pick, H. L., \& Ikeda, K. (1965). Visual capture produced by prism spectacles. Psychonomic Science, 
2(1-12), 215-216. https://doi.org/10.3758/BF03343413

Hogendoorn, H., Kammers, M. P. M., Carlson, T. a., \& Verstraten, F. a J. (2009). Being in the dark about your hand: Resolution of visuo-proprioceptive conflict by disowning visible limbs. Neuropsychologia, 47, 2698-2703. https://doi.org/10.1016/j.neuropsychologia.2009.05.014

Ilmoniemi, R. J., Virtanen, J., Ruohonen, J., Karhu, J., Aronen, H. J., Näätänen, R., \& Katila, T. (1997). Neuronal responses to magnetic stimulation reveal cortical reactivity and connectivity. NeuroReport, 8(16), 3537-3540. https://doi.org/10.1097/00001756-199711100-00024

Kanai, R., Muggleton, N. G., \& Walsh, V. (2008). TMS over the intraparietal sulcus induces perceptual fading. Journal of Neurophysiology, 100, 3343-3350. https://doi.org/10.1152/jn.90885.2008

Komatsu, H. (2006). The neural mechanisms of perceptual filling-in. Nature Reviews Neuroscience, 7(3), 220 231. https://doi.org/10.1038/nrn1869

Lewis, J. W., \& Van Essen, D. C. (2000). Corticocortical connections of visual, sensorimotor, and multimodal processing areas in the parietal lobe of the macaque monkey. Journal of Comparative Neurology, 428(1), 112-137. https://doi.org/10.1002/1096-9861(20001204)428:1<112::AIDCNE8>3.0.CO;2-9

Limanowski, J., \& Blankenburg, F. (2016). Integration of Visual and Proprioceptive Limb Position Information in Human Posterior Parietal, Premotor, and Extrastriate Cortex. Journal of Neuroscience, 36(9), 2582-2589. https://doi.org/10.1523/JNEUROSCI.3987-15.2016

Limanowski, J., \& Blankenburg, F. (2017). Posterior parietal cortex evaluates visuoproprioceptive congruence based on brief visual information. Scientific Reports, 7(1), 16659. https://doi.org/10.1038/s41598-017-16848-7

Macaluso, E., Frith, C. D., \& Driver, J. (2000). Modulation of Human Visual Cortex by Crossmodal Spatial Attention. Science (New York, NY), 289(5482), 1206-1208. https://doi.org/10.1126/science.289.5482.1206

Macaluso, E., Frith, C. D., \& Driver, J. (2002). Crossmodal spatial influences of touch on extrastriate visual areas take current gaze direction into account. Neuron, 34(4), 647-658. https://doi.org/10.1016/S0896-6273(02)00678-5

Maravita, A., Spence, C., \& Driver, J. (2003). Multisensory integration and the body schema: Close to hand and within reach. Current Biology, 13(13), 531-539. https://doi.org/10.1016/S09609822(03)00449-4

Martinez-Conde, S., Macknik, S. L., \& Hubel, D. H. (2004). The role of fixational eye movements in visual perception. Nature Reviews Neuroscience, 5(3), 229-240. https://doi.org/10.1038/nrn1348

Martinez-Conde, S., Otero-Millan, J., \& MacKnik, S. L. (2013). The impact of microsaccades on vision: Towards a unified theory of saccadic function. Nature Reviews Neuroscience, 14(2), 83-96. https://doi.org/10.1038/nrn3405

Mendola, J. D., Conner, I. P., Sharma, S., Bahekar, a, \& Lemieux, S. (2006). fMRI Measures of perceptual filling-in in the human visual cortex. Journal of Cognitive Neuroscience, 18(3), 363-375. https://doi.org/10.1162/jocn.2006.18.3.363

Murray, S. O., Boyaci, H., \& Kersten, D. (2006). The representation of perceived angular size in human primary visual cortex. Nature Neuroscience, 9(3), 429-434. https://doi.org/10.1038/nn1641 
Rademaker, R. L., Wu, D. A., Bloem, I. M., \& Sack, A. T. (2014). Intensive tool-practice and skillfulness facilitate the extension of body representations in humans. Neuropsychologia, 56(1), 196-203. https://doi.org/10.1016/j.neuropsychologia.2014.01.011

Ramos-Estebanez, C., Merabet, L. B., Machii, K., Fregni, F., Thut, G., Wagner, T. A., .. Pascual-Leone, A. (2007). Visual Phosphene Perception Modulated by Subthreshold Crossmodal Sensory Stimulation. Journal of Neuroscience, 27(15), 4178-4181. https://doi.org/10.1523/JNEUROSCI.5468-06.2007

Riggs, L. A., Ratliff, F., Cornsweet, J. C., \& Cornsweet, T. N. (1953). The disappearance of steadily fixated visual test objects. Journal of the Optical Society of America, 43(6), 495-501.

Rossi, S., Hallett, M., Rossini, P. M., \& Pascual-Leone, A. (2012). Safety, ethical considerations, and application guidelines for the use of transcranial magnetic stimulation in clinical practice and

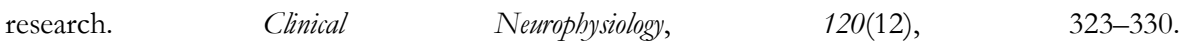
https://doi.org/10.1016/j.clinph.2009.08.016.Rossi

Sakata, H., Takaoka, Y., Kawarasaki, A., \& Shibutani, H. (1973). Somatosensory properties of neurons in the superior parietal cortex (area 5) of the rhesus monkey. Brain Research, 64(C), 85-102. https://doi.org/10.1016/0006-8993(73)90172-8

Sasaki, Y., \& Watanabe, T. (2004). The primary visual cortex fills in color. Proceedings of the National Academy of Sciences of the United States of America, 101(52), 18251-18256. https://doi.org/10.1073/pnas.0406293102

Shimojo, S., Kamitani, Y., \& Nishida, S. (2001). Afterimage of perceptually filled-in surface. Science, 293, 1677-80. https://doi.org/10.1126/science.1060161

Sperandio, I., Chouinard, P. A., \& Goodale, M. A. (2012). Retinotopic activity in V1 reflects the perceived and not the retinal size of an afterimage. Nature Neuroscience, 15(4), 540-542. https://doi.org/10.1038/nn.3069

Troxler, D. I. P. V. (1804). Über das Verschwinden gegebener Gegenstände innerhalb unseres Gesichtskreises. Ophthalmologische Bibliothek, 2(2), 1-53.

Wald, G. (1955). The photoreceptor process in vision. American Journal of Opbthalmology, 40(5), 18-41. 
Chapter 8
General Discussion 

This thesis set out to gain a further understanding in a range of topics spanning from the link between emotions and actions, to bodily self-consciousness, and into methodological challenges when studying these questions.

\section{Overview of the findings}

Part 1 of the thesis addressed the question whether areas related to the observation of actions are crucial in emotion body processing. In Chapter 2 we focused on one well established area involving the observation of actions, namely Inferior Parietal Lobule (IPL), and tested its causal relevance in emotion body perception. Participants received online TMS stimulation over either IPL, extrastriate body area, early visual cortex or sham stimulation, while completing a delayed match-to-sample task. This task involved briefly presented static images of body postures depicting either a fearful or a neutral stance, and minimal postural changes between the sample and the probe had to be detected. Critically, behavioural performance improved selectively for the fearful postures after stimulation of IPL, but not any of the other stimulation sites. The next step was to see with which remote areas IPL interacts under circumstances of emotion body perception. In Chapter 3 we used an offline combination of cTBS and fMRI to answer this question. Additionally, we wanted to investigate another action related area with regard to emotion body processing, namely ventral premotor cortex (PMv). Participants completed three separate sessions, in which they either received cTBS over IPL, cTBS over PMv or no stimulation, before entering the scanner. We then investigated condition and stimulation specific influences on the BOLD signal by presenting the participants with blocks of dynamic stimuli depicting either neutral or angry whole body actions actions. Interestingly, results showed an interaction between stimulus condition and stimulation site in the amygdala, a wellknown site for emotion processing. This interaction revealed that whereas at baseline no distinction was being made between angry and neutral actions in the amygdala, this difference became highly significant after stimulation of either IPL or PMv. Besides this finding, interactions between stimulation site and stimulus condition were found in a host of other regions related to both action observation and action preparation.

Part 2 of the thesis focused on the motor system. Firstly, in Chapter 4 we were interested in the relation between the state of the motor system and emotion body perception. Specifically, we were interested to see whether changes in corticospinal excitability could be observed in both conscious and unconscious perception of threatening body postures. To this end we used a combination of assessment of the amplitude of Motor Evoked Potentials (MEPs) with a Continuous Flash Suppression (CFS) task. In this CFS task participants were presented with static images of threatening (angry or fearful) or neutral bodies, or objects, either inside or outside of their conscious awareness. While these stimuli were being presented, single pulses of TMS were applied at different time points after stimulus onset to evoke MEPs. The only significant finding was an early effect of increased MEP amplitude in response to angry bodies compared to objects in a conscious viewing condition. This lead us to the question about high variability in MEPs and possible ways to reduce this. Therefore, in Chapter $\mathbf{5}$ we specifically targeted one possible underlying cause of MEP variability, namely oscillatory power and phase. By using an online combination of transcranial alternating current stimulation (tACS) and single pulse TMS, we looked into the influence of individual alpha or individual beta peak frequency of the tACS signal on MEP amplitude. We found no offline changes as a result of the tACS entrainment in either the alpha or beta condition. When looking at the influence of the ongoing phase of the entraining tACS signal however, we were able to significantly fit a one 
cycle sinusoid over the normalized MEP amplitude across eight different phase bins in the beta, but not the alpha or sham, condition. We further extended these findings by showing this phase dependence was specific to participants showing a low intrinsic beta frequency.

Lastly, part 3 of the thesis related to questions surrounding bodily self-consciousness. In Chapter $\mathbf{6}$ we addressed the question whether perception of emotions in the environment can alter the experience of body ownership. We tested this question by using a well-established paradigm for measuring and manipulating body ownership, namely the Rubber Hand Illusion (RHI). We combined this illusion with the presentation of emotional vocalizations or control stimuli (no sound or non-vocal sounds) and measured the influence on the subjective experience of ownership over the rubber hand and proprioceptive drift towards the rubber hand. We found a significant increase in the proprioceptive drift towards the rubber hand, but not the subjective experience of ownership, when participants were presented with affective vocalizations compared to the control conditions. In a second experiment, we ruled out that this was simply the result of being presented with vocalizations per se by adding a neutral vocalizations condition. We replicated the increase in proprioceptive drift in response to affective vocalizations. Furthermore, we ruled out that the effect was caused by being presented with voices in general, as there was no difference between a no sound and neutral vocalizations condition. In Chapter 6 we were interested in another perceptual illusion, namely positive afterimages. Specifically, we were interested to see whether the fading of afterimages of the hands, which stems from a conflict between vision and proprioception, is the result of active inhibition of early visual cortex (EVC). To test this hypothesis, we assessed whether the amount of perceptual fading of the hand could be altered by online TMS stimulation of EVC overlapping with the phosphene location of the hand afterimage. The results showed that triple pulse stimulation lead to gestalt-like reappearance of the faded afterimage of the hand, which did not occur in the single pulse or sham TMS conditions. However, this effect was evident for both the left and the right hand, despite that stimulation only was applied to the right hemisphere (corresponding to the left hemifield).

\section{Importance of stimulus selection when probing emotion specificity in action related areas}

In Chapters 2, 3, and 4 we investigated if action observation and motor areas dissociate between neutral and emotional (specifically threatening) whole body postures. In terms of stimulus material, for chapter 2 we used static images showing fearful or neutral postures, but importantly these postures were matched for implied motion. In chapter 3 , thanks to the offline stimulation experimental design, we were able to use dynamic stimuli that portrayed either angry or neutral actions (which depicted an actor bringing up their hand to cough in it), whereas in chapter 4 we were limited to the use of static whole body images of neutral or threatening (both angry and fear) body images to be able to combine them with a continuous flash suppression task. When trying to demonstrate that action areas dissociate between actions that are neutral or portraying an emotion, stimulus selection is crucial to be able to draw valid conclusions. Therefore, in the following paragraphs several important aspects of stimulus selection will be discussed.

One prominent stimulus feature that needs to be considered for the study of emotion specificity in action-related areas is the amount of motion implied/displayed in a whole body action. The importance of this particular feature was demonstrated by the work of Borgomaneri, Gazzola, \& Avenanti (2012), in which they tried to assess whether the amplitude of TMS- 
induced motor evoked potentials was modulated by emotional versus neutral whole body actions. Interestingly, it was amount of motion, rather than valence, that lead to an increase in MEP amplitude. Additionally, the emotional modulation of MEP amplitude was specific to dynamic stimuli compared to static ones, emphasizing the role of motion in emotion processing. In later studies, an influence of emotion on excitability of the motor cortex was demonstrated, depending on time of stimulation and taking intracortical facilitation as an outcome measure (Borgomaneri, Gazzola, \& Avenanti, 2014; Borgomaneri, Vitale, \& Avenanti, 2015; Borgomaneri, Vitale, Gazzola, \& Avenanti, 2015), but nonetheless underlining the importance of matching (implied) motion between neutral and emotional stimuli. Recent work by Goldberg, Christensen, Flash, Giese, \& Malach (2015) addressed challenges in controlling for inherent differences between emotion and neutral whole body actions in terms of motion by creating avatars in which gait was matched across different emotional states and a neutral control. In their fMRI study, the authors found emotion specific activity in the dorsal action stream. Interestingly though, results were stronger when looking at perceived, rather than expressed valence, showing that also the experimental task is not trivial.

Besides matching neutral and emotional actions for amount of motion, it is also crucial to consider the content of the action. The action observation literature suggests a dissociation is made in action observation areas between meaningful and meaningless actions (e.g. Decety et al., 1997; Newman-Norlund, Schie, Hoek, Cuijpers, \& Bekkering, 2009). Therefore, taking meaningless actions as a neutral control will not lead to valuable insights into emotion specificity of action areas. Matching emotional and neutral actions in content is not straightforward, but an example comes from the work by Pichon, de Gelder, \& Grezes (2008). In this fMRI study, the authors kept the content of the action constant while varying its valence, by presenting participants with videos of actors opening a door in either a neutral or an angry state. Their results show that dynamic whole body expressions of anger in particular, activate several areas within the action observation network.

Another dimension to take into consideration when comparing neutral and emotional whole body actions is how naturalistic the stimulus is. As easily identifiable as stimuli like point-likedisplays or avatars might be, they are hardly comparable to what our brain actually deals with in realistic circumstances. So far, only one fMRI study tried to investigate the effect of whole body emotions expressed in a natural context. Goldberg, Preminger, \& Malach (2014) used short audio-visual clips taken from movies in which an emotion was expressed, and their results demonstrated emotion-arousal specific activity in parietal areas that lie in the dorsal action stream. Importantly though, given this stimulus set it is impossible to isolate effects that are specific to the emotions per se, or other factors that co-vary with the emotion.

\section{Transcranial Magnetic Stimulation in Cognitive Neuroscience}

Most of the research featured in this thesis used TMS as a research method to either influence the state of brain areas of interest (by using online sp/tpTMS or offline cTBS) or as a read out for the state of the motor systems (by measuring motor evoked potentials). TMS was used in combination with behavioural measures, fMRI, tACS and EMG. In Chapter 2 and Chapter 7 online rTMS was used to shortly disrupt a brain area of interest and observe changes in behavior or perception, in Chapter 3 offline cTBS was combined with fMRI to assess conditionspecific network effects of stimulation, and lastly in Chapter $\mathbf{4}$ and Chapter $\mathbf{5}$ single pulse TMS was used to evoke MEPs and get a condition-specific read out of the state of the motor system. This speaks to the versatility of TMS as a research tool and its value within the field of 
cognitive neuroscience. However, as with every method, TMS has its own limitations. It is becoming clearer that one particular stimulation protocol might yield one result in one individual, no result in another, and even an opposing response in yet another individual. Not only this, but results obtained for one individual on one particular day might not replicate to the next week. The source of much of this variability is yet to be discovered, but the field is uncovering more and more about individual responses to TMS. The protocols used in the current thesis will now be discussed and placed within the current knowledge of TMS efficacy and its contributing factors.

\section{State dependence of online stimulation protocols}

In Chapter 2 we assessed the influence of online tpTMS by analyzing changes in behavioural accuracy that result from stimulation of different areas. Even though we hypothesized that indeed IPL should lead to changes in behavioural accuracy specific to the fear condition, the finding that this change was in the positive direction was somewhat surprising. When viewing TMS in its classical sense, which is as a tool for disruption of brain areas to test their causal relevance, an improvement in behaviour might not be the most intuitive finding. One thing that is becoming increasingly clear, however, is that simply speaking about TMS protocols in terms of excitation versus inhibition might paint an overly simplistic picture, and multiple factors play into how the brain is actually influenced by stimulation. For example, several studies have found that stimulation of the same area can have an effect in either direction (inhibition/facilitation) just by changing the stimulation intensity from sub- to supra-threshold (Abrahamyan, Clifford, Arabzadeh, \& Harris, 2011; Abrahamyan, Clifford, Arabzadeh, \& Harris, 2015). It seems from these studies that whereas supra-threshold stimulation is inhibitory, sub-threshold stimulation can have facilitatory effects. It has to be noted that these studies were executed in the visual system, for which the threshold can be measured by means of elicited phosphenes. For non-motor and non-visual brain areas, stimulation intensity is usually determined based on individual motor or phosphene threshold, but there is no way of validating whether for these brain areas that constitutes sub- or suprathreshold stimulation. This also makes it difficult to determine in regions such as the parietal cortex whether a certain stimulation intensity should lead to inhibition or facilitation.

Another crucial factor for determining the direction of TMS effects is the state of the brain area at the time of stimulation. State-dependence of TMS was first demonstrated by means of adaption; for example, when participants are first adapted to a uniform red colour, subsequently elicited phosphenes will take on the same colour properties as the adaptation stimulus (Silvanto, Muggleton, Cowey, \& Walsh, 2007). A recent paper argued for a framework in which state dependence and the direction of TMS effects is accounted for (Silvanto \& Cattaneo, 2017). They propose intensity ranges for facilitatory and inhibitory effects, which are determined by the excitability of the underlying neurons. The model suggests that neuronal populations that are either tuned or not tuned to a particular stimulus have different excitability ranges, and low or high intensity TMS stimulation might therefore target them in opposing ways. Because of the influence that brain state can have on the direction of TMS effects, one suggestion for improving specificity of TMS can be accomplished through having participants perform a task before the application of the stimulation, which at least in part controls for the brain state of all participants (Romei, Thut, \& Silvanto, 2016). Such pre-conditioning could also be accomplished through electrical current stimulation (Lang et al., 2004; Siebner et al., 2004). 


\section{Theta Burst Stimulation}

Theta Burst Stimulation (TBS) is an offline protocol in which bursts of pulses (triplets at $50 \mathrm{~Hz}$ ) are repeated in a theta rhythm $(5 \mathrm{~Hz})$. This can either be delivered continuously (continuous theta burst stimulation, or cTBS) or 2 seconds of stimulation spaced with 8 seconds intervals of no stimulation (intermittent theta burst stimulation, or iTBS) (Huang, Edwards, Rounis, Bhatia, \& Rothwell, 2005). Whereas cTBS is an inhibitory protocol, iTBS enhances cortical excitability, and this inhibition and excitation is linked to processes similar to long-term depression (LTD) and long-term potentiation (LTP) (Di Lazzaro et al., 2005). TBS protocols have proven useful markers of plasticity (Pascual-Leone, Tormos, \& Keenan, 1998), and thanks to their long-lasting offline effects (up to 40 minutes after stimulation) they are also particularly suitable for combination with other neuroimaging techniques such as EEG or fMRI. Nonetheless, TBS protocols can suffer from large inter- and intra-subject variability. When evaluating TBS protocols on the group level, excitatory and inhibitory effects of M1 stimulation on MEP amplitude over time are well established (see e.g. Wischnewski \& Schutter (2015) for a recent meta-analysis). The story becomes more complicated when evaluating TBS efficacy on the individual level. For example, one large scale study reported that as many as $57 \%$ of the participants either failed to show the expected iTBS effects, or even show a reversal of the expected effects (López-Alonso, Cheeran, Río-Rodríguez, \& Fernández-Del-Olmo, 2014). Furthermore, using cluster analysis they were able to identify distinct groups of responders and non-responders. A separate study used cluster analysis to similarly demonstrate the existence of such subpopulations of responders and non-responders for cTBS stimulation (Jannati, Block, Oberman, Rotenberg, \& Pascual-Leone, 2017).

The story of TBS efficacy becomes even more complicated when considering that most of the work has been done within the motor system. This is logical considering the clear read-out that MEPs can provide for the motor system, which is mostly absent in other areas of the brain. One option for assessing TBS induced changes in other areas of the brain is the BOLD response measured with fMRI. This outcome measure has similarly painted a picture of individual differences in the direction of TBS effects. For example, work by Valchev, Gazzola, Avenanti, \& Keysers (2016) failed to show consistent local effects of cTBS over BA 1/2 in an ROI analysis, caused by some participant showing decreases in BOLD signal while others showed an increase, despite the fact that cTBS is thought to be an inhibitory protocol.

Trying to understand TBS efficacy and variability, there are two important levels to consider; the first is variability stemming from the brain being stimulated, and the second source of variability stemming from the technical application of the protocol.

When looking at factors stemming from the participant, several factors have been found to influence how TBS is received. So far, influential factors that have been studied include baseline M1-premotor resting-state functional connectivity (Cárdenas-Morales et al., 2014; Nettekoven et al., 2015), genetics (Cheeran et al., 2008; Jannati et al., 2017), and muscle pre-activations (Goldsworthy, Müller-Dahlhaus, Ridding, \& Ziemann, 2014). See Ridding \& Ziemann (2010) for a review on factors that influence changes in cortical plasticity induced by NIBS protocols. When on the other hand examining variability stemming from the technical application of TBS, there are also multiple factors to be considered. Most studies that apply TBS conform to the original paper published by Huang et al. (2005) and use a stimulation intensity of $80 \%$ of active motor threshold. However, such fixed intensity does not take into account differences in scalp 
to cortex distance, and one may also wonder how well this intensity translates to non-motor regions. Besides stimulation intensity, other factors that are being investigated are the influence of stimulation duration (Gamboa, Antal, Moliadze, \& Paulus, 2010; Nettekoven et al., 2014), stimulation frequencies (e.g. modified cTBS, see Goldsworthy, Pitcher, \& Ridding, 2012), and current direction (Talelli, Cheeran, Teo, \& Rothwell, 2007).

Taking all of the above into account, by no means do TBS protocols lose their value. The current understanding of variability in TBS effects can be applied to strengthen experimental designs; practically speaking this can influence what constitutes an appropriate sample size for TBS experiments, if at all possible quantification of the efficacy and direction of the TBS effect in each participant should be measured (e.g. by means of TEPs), and this data could provide valuable information to drive data analysis (see e.g. Valchev et al., 2016). Optimization of stimulation protocols, EEG feedback, modifications of pulse shape, as well as more sensitive outcome measures will also aid in the reduction of TBS response variability (Guerra, LópezAlonso, Cheeran, \& Suppa, 2017; Huang et al., 2017). One study already demonstrated enhanced cTBS effects by applying cTBS aligned with the trough of a tACS entraining signal at the endogenous alpha frequency (Goldsworthy, Vallence, Yang, Pitcher, \& Ridding, 2015). Future research will hopefully also further inform decisions on appropriate stimulation intensity and duration, as well as how well findings in the motor cortex actually translate to other non-motor brain regions.

\section{Using Motor Evoked Potentials to assess the state of the motor system}

The amplitude of Motor Evoked Potentials provide a read-out for corticospinal excitability, which is not only useful in research, but also clinical settings. However, anyone who has experience working with the measurement of MEPs will attest to the large variability in this measure, which not only varies between participants, between sessions, but also from pulse to pulse. The goal of Chapter 5 was to investigate the influence of the power and phase of an entraining tACS signal. We found that the amplitude of an MEP was modulated by the phase of an ongoing tACS signal in a beta frequency. This is in line with previous studies showing similar effects for the phase of beta tACS (Guerra et al., 2016; Nakazono, Ogata, Kuroda, \& Tobimatsu, 2016; Raco, Bauer, Tharsan, \& Gharabaghi, 2016). A direct involvement of intrinsic neuronal oscillatory phase has been demonstrated by combined EEG-TMS (Schulz, Übelacker, Keil, Müller, \& Weisz, 2014; Zrenner, Desideri, Belardinelli, \& Ziemann, 2017). Other intrinsic sources of MEP variability stem from age, with older subjects showing higher MEP variability, as well as females showing higher MEP variability compared to males (Pitcher, Ogston, \& Miles, 2003).

As is also the case with variability observed in response to TBS protocols, for MEP variability we can similarly identify both internal physiological sources, such as neuronal oscillations, as well as external sources related to the application of TMS. One such external source that is important to keep in mind while designing an experiment is the inter-pulse interval (IPI). One study demonstrated that longer IPIs (10sec) resulted in larger MEP amplitudes than short IPIs (4sec)(Vaseghi, Zoghi, \& Jaberzadeh, 2015). Another important practical matter when acquiring MEP data is ensuring an appropriate number of MEPs per condition of interest. Recent work by Goldsworthy, Hordacre, \& Ridding (2016) suggests the optimal number of MEPs per condition is 20-30 for ensuring optimal intra- and intersession reliability, but going beyond 30 trials per condition provides no additional information. 
Ultimately, we will have to accept that there are several factors that contribute to MEP variability which we can have no direct control over. Nonetheless, a better understanding of all sources of MEP variability will lead to the ability to regulate certain factors that we can exert control over, leading to a reduction of MEP variability. 


\section{References}

Abrahamyan, A., Clifford, C. W. G., Arabzadeh, E., \& Harris, J. A. (2011). Improving Visual Sensitivity with Subthreshold Transcranial Magnetic Stimulation. Journal of Neuroscience, 31(9), 3290-3294. https://doi.org/10.1523/JNEUROSCI.6256-10.2011

Abrahamyan, A., Clifford, C. W. G., Arabzadeh, E., \& Harris, J. A. (2015). Low intensity TMS enhances perception of visual stimuli. Brain Stimulation, 8(6), 1175-1182. https://doi.org/10.1016/j.brs.2015.06.012

Borgomaneri, S., Gazzola, V., \& Avenanti, A. (2012). Motor mapping of implied actions during perception of emotional body language. Brain Stimulation, 5(2), 70-6. https://doi.org/10.1016/j.brs.2012.03.011

Borgomaneri, S., Gazzola, V., \& Avenanti, A. (2014). Transcranial magnetic stimulation reveals two functionally distinct stages of motor cortex involvement during perception of emotional body language. Brain Structure \& Function. https://doi.org/10.1007/s00429-014-0825-6

Borgomaneri, S., Vitale, F., \& Avenanti, A. (2015). Early changes in corticospinal excitability when seeing fearful body expressions. Scientific Reports, 5, 14122. https://doi.org/10.1038/srep14122

Borgomaneri, S., Vitale, F., Gazzola, V., \& Avenanti, A. (2015). Seeing fearful body language rapidly freezes the observer's motor cortex. Cortex, 65, 232-245. https://doi.org/10.1016/j.cortex.2015.01.014

Cárdenas-Morales, L., Volz, L. J., Michely, J., Rehme, A. K., Pool, E. M., Nettekoven, C., ... Grefkes, C. (2014). Network connectivity and individual responses to brain stimulation in the human motor system. Cerebral Cortex, 24(7), 1697-1707. https://doi.org/10.1093/cercor/bht023

Cheeran, B., Talelli, P., Mori, F., Koch, G., Suppa, A., Edwards, M., ... Rothwell, J. C. (2008). A common polymorphism in the brain-derived neurotrophic factor gene (BDNF) modulates human cortical plasticity and the response to rTMS. Journal of Physiology, 586(23), 5717-5725. https://doi.org/10.1113/jphysiol.2008.159905

Decety, J., Grèzes, J., Costes, N., Perani, D., Jeannerod, M., Procyk, E., ... Fazio, F. (1997). Brain activity during observation of actions. Influence of action content and subject's strategy. Brain, (120), 1763-1777.

Di Lazzaro, V., Pilato, F., Saturno, E., Oliviero, A., Dileone, M., Mazzone, P., ... Rothwell, J. C. (2005). Theta-burst repetitive transcranial magnetic stimulation suppresses specific excitatory circuits in the human motor cortex. Journal of Physiology, 565(3), 945-950. https://doi.org/10.1113/jphysiol.2005.087288

Gamboa, O. L., Antal, A., Moliadze, V., \& Paulus, W. (2010). Simply longer is not better: Reversal of theta burst after-effect with prolonged stimulation. Experimental Brain Research, 204(2), 181-187. https://doi.org/10.1007/s00221-010-2293-4

Goldberg, H., Christensen, A., Flash, T., Giese, M. A., \& Malach, R. (2015). Brain activity correlates with emotional perception induced by dynamic avatars. Neurolmage, 122, 306-317. https://doi.org/10.1016/j.neuroimage.2015.07.056

Goldberg, H., Preminger, S., \& Malach, R. (2014). The emotion-action link? Naturalistic emotional stimuli preferentially activate the human dorsal visual stream. NeuroImage, 84, 254-64. 
https://doi.org/10.1016/j.neuroimage.2013.08.032

Goldsworthy, M. R., Hordacre, B., \& Ridding, M. C. (2016). Minimum number of trials required for within- and between-session reliability of TMS measures of corticospinal excitability. Neuroscience, 320, 205-209. https://doi.org/10.1016/j.neuroscience.2016.02.012

Goldsworthy, M. R., Müller-Dahlhaus, F., Ridding, M. C., \& Ziemann, U. (2014). Inter-subject variability of LTD-like plasticity in human motor cortex: A matter of preceding motor activation. Brain Stimulation, 7(6), 864-870. https://doi.org/10.1016/j.brs.2014.08.004

Goldsworthy, M. R., Pitcher, J. B., \& Ridding, M. C. (2012). A comparison of two different continuous theta burst stimulation paradigms applied to the human primary motor cortex. Clinical Neurophysiology, 123(11), 2256-2263. https://doi.org/10.1016/j.clinph.2012.05.001

Goldsworthy, M. R., Vallence, A.-M., Yang, R., Pitcher, J. B., \& Ridding, M. C. (2015). Combined transcranial alternating current stimulation and cTBS: a novel approach for neuroplasticity induction. European Journal of Neuroscience, 43, n/a-n/a. https://doi.org/10.1111/ejn.13142

Guerra, A., López-Alonso, V., Cheeran, B., \& Suppa, A. (2017). Variability in non-invasive brain stimulation studies: Reasons and results. Neuroscience Letters, (December), 0-1. https://doi.org/10.1016/j.neulet.2017.12.058

Guerra, A., Pogosyan, A., Nowak, M., Tan, H., Ferreri, F., Di Lazzaro, V., \& Brown, P. (2016). Phase Dependency of the Human Primary Motor Cortex and Cholinergic Inhibition Cancelation During Beta tACS. Cerebral Cortex, 26(10), 3977-3990. https://doi.org/10.1093/cercor/bhw245

Huang, Y.-Z., Lu, M.-K., Antal, A., Classen, J., Nitsche, M., Ziemann, U., ... Rothwell, J. (2017). Plasticity induced by non-invasive transcranial brain stimulation: A position paper. Clinical Neurophysiology, 128(11), 2318-2329. https://doi.org/10.1016/j.clinph.2017.09.007

Huang, Y. Z., Edwards, M. J., Rounis, E., Bhatia, K. P., \& Rothwell, J. C. (2005). Theta burst stimulation of the human motor cortex. Neuron, 45(2), 201-206. https://doi.org/10.1016/j.neuron.2004.12.033

Jannati, A., Block, G., Oberman, L. M., Rotenberg, A., \& Pascual-Leone, A. (2017). Interindividual variability in response to continuous theta-burst stimulation in healthy adults. Clinical Neurophysiology, 128(11), 2268-2278. https://doi.org/10.1016/j.clinph.2017.08.023

Lang, N., Siebner, H. R., Ernst, D., Nitsche, M. A., Paulus, W., Lemon, R. N., \& Rothwell, J. C. (2004). Preconditioning with transcranial direct current stimulation sensitizes the motor cortex to rapidrate transcranial magnetic stimulation and controls the direction of after-effects. Biological Psychiatry, 56(9), 634-639. https://doi.org/10.1016/j.biopsych.2004.07.017

López-Alonso, V., Cheeran, B., Río-Rodríguez, D., \& Fernández-Del-Olmo, M. (2014). Inter-individual variability in response to non-invasive brain stimulation paradigms. Brain Stimulation, 7(3), 372380. https://doi.org/10.1016/j.brs.2014.02.004

Nakazono, H., Ogata, K., Kuroda, T., \& Tobimatsu, S. (2016). Phase and frequency-dependent effects of transcranial alternating current stimulation on motor cortical excitability. PLoS ONE, 11(9), 113. https://doi.org/10.1371/journal.pone.0162521

Nettekoven, C., Volz, L. J., Kutscha, M., Pool, E.-M., Rehme, A. K., Eickhoff, S. B., ... Grefkes, C. (2014). Dose-Dependent Effects of Theta Burst rTMS on Cortical Excitability and Resting-State 
Connectivity of the Human Motor System. Journal of Neuroscience, 34(20), 6849-6859. https://doi.org/10.1523/JNEUROSCI.4993-13.2014

Nettekoven, C., Volz, L. J., Leimbach, M., Pool, E. M., Rehme, A. K., Eickhoff, S. B., ... Grefkes, C. (2015). Inter-individual variability in cortical excitability and motor network connectivity following multiple blocks of rTMS. NeuroImage, 118, 209-218. https://doi.org/10.1016/j.neuroimage.2015.06.004

Newman-Norlund, R., Schie, H. T. Van, Hoek, M. E. C. Van, Cuijpers, R. H., \& Bekkering, H. (2009). The role of inferior frontal and parietal areas in differentiating meaningful and meaningless objectdirected actions. Brain Research, 1315, 63-74. https://doi.org/10.1016/j.brainres.2009.11.065

Pascual-Leone, A., Tormos, J., \& Keenan, J. (1998). Study and modulation of human cortical excitability with transcranial magnetic stimulation. Journal of Clinical. Retrieved from http://journals.lww.com/clinicalneurophys/Abstract/1998/07000/Study_and_Modulation_of_ Human_Cortical.5.aspx

Pichon, S., de Gelder, B., \& Grezes, J. (2008). Emotional modulation of visual and motor areas by dynamic body expressions of anger. Social Neuroscience, 3(3-4), 199-212. https://doi.org/10.1080/17470910701394368

Pitcher, J. B., Ogston, K. M., \& Miles, T. S. (2003). Age and sex differences in human motor cortex inputoutput characteristics. The Journal of Physiology, 546(2), 605-613. https://doi.org/10.1113/jphysiol.2002.029454

Raco, V., Bauer, R., Tharsan, S., \& Gharabaghi, A. (2016). Combining TMS and tACS for Closed-Loop Phase-Dependent Modulation of Corticospinal Excitability: A Feasibility Study. Frontiers in Cellular Neuroscience, 10(May), 1-8. https://doi.org/10.3389/fncel.2016.00143

Ridding, M. C., \& Ziemann, U. (2010). Determinants of the induction of cortical plasticity by non-invasive brain stimulation in healthy subjects. The Journal of Physiology, 588(13), 2291-2304. https://doi.org/10.1113/jphysiol.2010.190314

Romei, V., Thut, G., \& Silvanto, J. (2016). Information-Based Approaches of Noninvasive Transcranial $\begin{array}{llll}\text { Brain Stimulation. Trends in Neurosciences, 39(11), 795. } & \text { 782 }\end{array}$ https://doi.org/10.1016/j.tins.2016.09.001

Schulz, H., Übelacker, T., Keil, J., Müller, N., \& Weisz, N. (2014). Now i am ready - Now i am not: The influence of pre-TMS oscillations and corticomuscular coherence on motor-evoked potentials. Cerebral Cortex, 24(7), 1708-1719. https://doi.org/10.1093/cercor/bht024

Siebner, H. R., Lang, N., Rizzo, V., Nitsche, M. A., Paulus, W., Lemon, R. N., \& Rothwell, J. C. (2004). Preconditioning of Low-Frequency Repetitive Transcranial Magnetic Stimulation with Transcranial Direct Current Stimulation: Evidence for Homeostatic Plasticity in the Human Motor Cortex. Journal of Neuroscience, 24(13), 3379-3385. https://doi.org/10.1523/JNEUROSCI.531603.2004

Silvanto, J., \& Cattaneo, Z. (2017). Common framework for "virtual lesion" and state-dependent TMS: The facilitatory/suppressive range model of online TMS effects on behavior. Brain and Cognition, 119(July), 32-38. https://doi.org/10.1016/j.bandc.2017.09.007

Silvanto, J., Muggleton, N. G., Cowey, A., \& Walsh, V. (2007). Neural adaptation reveals state-dependent effects of transcranial magnetic stimulation. European Journal of Neuroscience, 25(6), 1874-1881. 
https://doi.org/10.1111/j.1460-9568.2007.05440.x

Talelli, P., Cheeran, B. J., Teo, J. T. H., \& Rothwell, J. C. (2007). Pattern-specific role of the current orientation used to deliver Theta Burst Stimulation. Clinical Neurophysiology, 118(8), 1815-1823. https://doi.org/10.1016/j.clinph.2007.05.062

Valchev, N., Gazzola, V., Avenanti, A., \& Keysers, C. (2016). Primary somatosensory contribution to action observation brain activity-combining fMRI and cTBS. Social Cognitive and Affective Neuroscience, 11(8), 1205-1217. https://doi.org/10.1093/scan/nsw029

Vaseghi, B., Zoghi, M., \& Jaberzadeh, S. (2015). Inter-pulse interval affects the size of single-pulse TMSinduced motor evoked potentials: A reliability study. Basic and Clinical Neuroscience, 6(1), 44-51.

Wischnewski, M., \& Schutter, D. J. L. G. (2015). Efficacy and time course of theta burst stimulation in healthy humans. Brain Stimulation, 8(4), 685-692. https://doi.org/10.1016/j.brs.2015.03.004

Zrenner, C., Desideri, D., Belardinelli, P., \& Ziemann, U. (2017). Real-time EEG-defined excitability states determine efficacy of TMS-induced plasticity in human motor cortex. Brain Stimulation. https://doi.org/10.1016/j.brs.2017.11.016 

Knowledge Valorization 

In this thesis, research is presented with a strong focus on questions relating to how the brain responds when observing threat in others. All of the studies presented in this thesis were sampled from a healthy student population, in order to gain a fundamental understanding of automatic responses to social threat. Using various neuroimaging methods, we tried to investigate how such responses are represented in the brain. This research has given us an insight into at which level of the visual processing stream a distinction is made between neutral and emotional postures, as well as the involvement of action areas in emotion perception. In the case of sampling participants from the healthy population, we would consider automatic responses to social threat as adaptive and from an evolutionary viewpoint beneficial for survival. A fundamental understanding of triggering defensive actions can first of all give us insight into the differences with certain patient groups that would fail to show such responses, and secondly eventually provide insight in how to tailor treatment for these patient groups.

\section{Automatic defensive responses to social threat; what if it goes wrong}

As important as appropriate responses to social threat are, they are far from a given. Take the example of freezing behavior; freezing is a beneficial behavioural response which occurs whenever presented threat is ambiguous, which could stem for example from uncertainty of the intention of someone else or from observing threat at a distance (Blanchard, Hynd, Minke, Minemoto, \& Blanchard, 2001). Freezing behavior allows for the scanning of the environment and making calculated decisions on how to proceed. Likewise, flight behaviour might be an optimal strategy when threat is close by and imminent. However, freeze and flight behaviours are not always the optimal defensive strategy when confronted with threat, and unnecessary or excessive freezing behaviour could be harmful rather than beneficial.

Excessive freezing and flight behaviour can be observed in anxiety disorders. Indeed, research has shown a positive correlation between the amount of freezing behaviour in response to aversive pictures and measured trait anxiety (Roelofs, Hagenaars \& Stins, 2010), as well as a demonstrating a link between freezing behavior and subjective anxiety, panic, and other symptoms of anxiety (Schmidt, Richey, Zvolensky, \& Maner, 2008). Similarly, one study presenting aversive pictures to either patients diagnosed with panic disorder, one of the six major anxiety disorders, or a group of control participants, showed heightened freezing responses in the patient group (Lopes et al., 2009). These are all examples of what is supposedly, from an evolutionary perspective, beneficial behavior leading to debilitating psychological disorders. When threat is misjudged to be omnipresent or inevitable, one might overexpress flight or freezing behavior, resulting for example in panic attacks or fear to leave the house. 
At the other end of the spectrum we find fight behavior. If you are in an immediately threatening situation, one response strategy might be to fight rather than flee from the threat. This might prove to be the best strategy whenever the threat is deemed as defeatable, or if outrunning the threat seems unlikely. As goes for freeze or flight tendencies, fight can be an extremely beneficial response given the right circumstances. However, if fight behavior is too easily elicited, aggressive behavior might be observed. Specifically, psychopathy, a disorder which is associated with an increase in instrumental aggression (Glenn \& Raine, 2009), has been linked to both increased fight tendencies, as well as reduced flight tendencies. Previous work has shown psychopathy patients fail to show avoidance tendencies towards angry faces that would normally be observed in healthy participants (von Borries, 2012).

Knowing that disorders such as anxiety and psychopathy might be partially explained through failure to show appropriate fight-flight-freeze responses to threat, how might this guide treatment plans? First of all, if we can establish a link between for example anxiety and symptoms rooted in avoidance of threat, treatment plans could be developed directly tackling the symptoms relating to this. For example, testosterone has been shown to facilitate social approach behaviours, and indeed administering testosterone to patients suffering from social anxiety disorders (SAD) increases approach tendencies towards presented angry faces (Dorien, Spinhoven \& Roelofs, 2016). In a healthy female population, it has been demonstrated that testosterone administration biases the amygdala towards more threat approach (Radke et al., 2015). In psychopathic individuals it has been demonstrated that there is less down regulation of amygdala though dorslolateral prefrontal cortex when control of emotional actions is required, an effect which is particularly evident in psychopathic individuals with high levels of endogenous testosterone levels (Volman et al, 2016).

With respect to our own work, specifically referring to Chapter 3, we demonstrated that TMS stimulation over action related areas (inferior parietal lobule and ventral premotor cortex) has the ability to alter emotion specific amygdala responses in healthy participants. Given that the amygdala has been placed in the center of mediating motor responses to threat (LeDoux \& Daw, 2018), it is of great interest for future research to explore whether such indirect mediation of amygdala could be beneficial for patient populations. This effect could be explored in two directions, firstly we demonstrated enhanced emotion sensitivity of the amygdala by means of an inhibitory protocol (continuous theta burst stimulation (cTBS)), something that could be of benefit in psychopathologies suffering from reduced emotion perception (e.g. psychopathy). On the other hand, it could be explored whether by use of excitatory protocols (such as intermittent theta burst stimulation (iTBS)) the reversed could be accomplished, i.e. reduced emotion sensitivity in the amygdala, something that could provide beneficial to patients suffering from anxiety disorders. 


\section{Optimization of Transcranial Magnetic Stimulation to increase clinical efficiency}

A second major theme in this thesis concerns the use of non-invasive brain stimulation, and how the use of this methodology can be improved. One feature avenue of improving efficiency of non-invasive brain stimulation is by individualizing the stimulation paramters. In this thesis we had a particular interest in the variability observed in outcome measures related typically obtained in brain stimulation studies. Specifically, Chapter 5 looked into some sources that may underlie variability that is observed when measuring Motor Evoked Potentials (MEPs) elicited with Transcranial Magnetic Stimulation (TMS). In this study we sought to understand whether the frequency and phase of an entraining transcranial alternating current stimulation (tACS) signal was applied at certain individualized frequencies. We found this to be the case when looking at the phase of an individualized beta signal entrainment. How can findings such as these be relevant in a broader perspective?

Importantly, TMS has proven of great value as a tool in cognitive neuroscience, but by now has proven itself equally indispensable within clinical settings (Lefaucheur et al, 2014, Wassermann \& Lisanby, 2001). The clinical relevance of TMS has been first and most convincingly demonstrated in the treatment of depression. In 2008 the application of repetitive TMS (rTMS) was approved by the U.S. Food and Drug Administration for the treatment of depression, particularly for patients who failed to benefit from medication. Last year, Zorgverzekeraars $\mathrm{Ne}$ derland deemed rTMS treatment for depression as scientifically proven to be beneficial, and in the near future Dutch health insurance will start covering the treatment costs. This treatment consists of applying a high frequency protocol $(10 \mathrm{~Hz})$ over the left dorsolateral prefrontal cortex daily, for the duration of 4-6 weeks. One great advantage of rTMS treatment is that side effects are relatively few, mostly relating to discomfort of the stimulation, whereas beneficial effects can last up to several months after the treatment has ended. Response rates to rTMS treatment are typically between 40-45\% (Fitzgerald, Hoy, Anderson \& Daskalakis, 2016).

Whereas these results are undoubtedly solid and a promising result, there is certainly room for improvement. This mainly becomes apparent when looking at the application of TMS treatment in other psychological or neurological disorders, for which either treatment success is low, or results of effectiveness are altogether lacking. Whereas there is now sufficient evidence for definite efficacy for the use of rTMS in the treatment of depression and pain, evidence for motor stroke and schizophrenia is still a more mixed, and efficiency for treatment of auditory hallucinations and tinnitus seems probable but is not yet irrefutably present (Lefaucheur et al., 2014). 
These mixed or altogether lacking findings could of course directly result from lack of effectiveness of TMS treatment for these particular disorders. However, given all the earlier mentioned benefits that TMS as a treatment method has, it is worthwhile exploring if the lack of evidence results from the fact that in these cases a 'one size fits all' approach is not fitting. Specifically, as we showed in Chapter 5 , variability of brain stimulation can be both explored and explained by oscillatory activity in the brain. This holds true for externally entrained oscillations, something which our data provides evidence for, but also holds true for intrinsic neuronal oscillations. Together, current data stemming from fundamental research, could and is being used to tailor brain stimulation treatment. Of particular interest for the future is moving towards individualized brain stimulation.

This idea of individualized brain stimulation for treatment is by no means a far-fetched idea reserved for a distant future (Thut et al., 2018). As a matter of fact, the amount of research on the topic is ever increasing, and just last month Nature news reported on the matter. In an article titled 'Brain-stimulation trials get personal to lift depression', it is outlined that variability in the effectiveness of brain stimulation treatment likely resulting from differences both within and between individuals. Most importantly an individual's current brain state and brain activity could be driving such differences. At the moment, two different trials trying to implement this knowledge in treatment for depression are running; one team at Monash university in Australia is specifically interested in the potential of using electroencephalography (EEG) in the online guiding of the stimulation frequencies used in tACS. Another team in Tübingen, Germany, is applying a similar methodology, however with an interest in TMS. This group recently already demonstrated in a healthy sample the potential of reducing variability online using EEG-triggered TMS, demonstrating efficacy both for Motor Evoked Potentials, as well as rTMS (Zrenner, Desideri, Belardinelli \& Ziemann, 2018).

The next step will be to apply this novel methodology to patient groups and see if indeed brain stimulation treatment can be optimized by means of individualized parameters. By applying brain stimulation in sync with ongoing intrinsic neuronal oscillations of the patient, and thus taking into account the current brain state of the patient, might radically change treatment efficacy. Additionally, efficacy of TMS treatment for disorders for which so far evidence was either weak, or lacking might be demonstrated. 


\section{References}

Blanchard, D. C., Hynd, A. L., Minke, K. A., Minemoto, T., \& Blanchard, R. J. (2001). Human defensive behaviors to threat scenarios show parallels to fear-and anxiety-related defense patterns of nonhuman mammals. Neuroscience \& Biobehavioral Reviewss, 25(7-8), 761-770.

Enter, D., Spinhoven, P., \& Roelofs, K. (2016). Dare to approach: single dose testosterone administration promotes threat approach in patients with social anxiety disorder. Clinical Psychological Sci ence, 4(6), 1073-1079.

Fitzgerald, P. B., Hoy, K. E., Anderson, R. J., \& Daskalakis, Z. J. (2016). A study of the pattern of re sponse to rTMS treatment in depression. Depression and anxiety, 33(8), 746-753.

Glenn, A. L., \& Raine, A. (2009). Psychopathy and instrumental aggression: Evolutionary, neurobiologi cal, and legal perspectives. International journal of law and psycbiatry, 32(4), 253-258.

LeDoux, J., \& Daw, N. D. (2018). Surviving threats: neural circuit and computational implications of a new taxonomy of defensive behaviour. Nature Reviens Neuroscience.

Lefaucheur, J. P., André-Obadia, N., Antal, A., Ayache, S. S., Baeken, C., Benninger, D. H., ... \& Devanne, H. (2014). Evidence-based guidelines on the therapeutic use of repetitive transcranial magnetic stimulation (rTMS). Clinical Neurophysiology, 125(11), 2150-2206.

Lopes, F. L., Azevedo, T. M., Imbiriba, L. A., Freire, R. C., Valença, A. M., Caldirola, D., ... \& Nardi, A. E. (2009). Freezing reaction in panic disorder patients associated with anticipatory anxiety. De pression and anxiety, 26(10), 917-921.

Radke, S., Volman, I., Mehta, P., van Son, V., Enter, D., Sanfey, A., ... \& Roelofs, K. (2015). Testosterone biases the amygdala toward social threat approach. Science advances, 1(5), e1400074.

Roelofs, K., Hagenaars, M. A., \& Stins, J. (2010). Facing freeze: Social threat induces bodily freeze in humans. Psychological Science, 21(11), 1575-1581.

Schmidt, N. B., Richey, J. A., Zvolensky, M. J., \& Maner, J. K. (2008). Exploring human freeze responses to a threat stressor. Journal of behavior therapy and experimental psychiatry, 39(3), 292-304.

Thut, G., Bergmann, T. O., Fröhlich, F., Soekadar, S. R., Brittain, J. S., Valero-Cabré, A., ... \& Ziemann, U. (2017). Guiding transcranial brain stimulation by EEG/MEG to interact with ongoing brain activity and associated functions: a position paper. Clinical Neurophysiology, 128(5), 843-857.

Volman, I., von Borries, A. K. L., Bulten, B. H., Verkes, R. J., Toni, I., \& Roelofs, K. (2016). Testosterone modulates altered prefrontal control of emotional actions in psychopathic offend ers. ENeuro, 3(1), ENEURO-0107. 
von Borries, A. K. L., Volman, I., de Bruijn, E. R. A., Bulten, B. H., Verkes, R. J., \& Roelofs, K. (2012). Psychopaths lack the automatic avoidance of social threat: relation to instrumental aggres sion. Psychiatry Research, 200(2), 761-766.

Wassermann, E. M., \& Lisanby, S. H. (2001). Therapeutic application of repetitive transcranial magnetic stimulation: a review. Clinical Neurophysiology, 112(8), 1367-1377.

Zrenner, C., Desideri, D., Belardinelli, P., \& Ziemann, U. (2018). Real-time EEG-defined excitability states determine efficacy of TMS-induced plasticity in human motor cortex. Brain Stimulation: Basic, Translational, and Clinical Research in Neuromodulation, 11(2), 374-389. 
Acknowledgements 

The past 4 years have been an incredible experience, and as much as it is cliché to say so; I would never have been where I am today without all the wonderful people that have supported me throughout the years.

I want to start by thanking both of my amazing supervisors. Bea, first of all I want to thank you for believing in me and giving me the opportunity to pursue a $\mathrm{PhD}$ in your group. Over the years you have continuously pushed me to try harder, but also to always take a new perspective on science/data/life. I have to admit that sometimes I would leave meetings with more questions than I had going into the meeting, but it has always proven to be fruitful. You also made me see that science does not have to be all work, and we had plenty of fun as well. I already apologise for making it a little sentimental now, but for me as a young female researcher in a world filled with imposter syndrome and loud opinionated men, it has truly inspired me to work for such an accomplished female scientist who has no fear of voicing her opinions. This is an experience that will stay with me the rest of my career.

Alex, thanks to you I have always felt as much part of the brain and emotion group as I did of the TMS group (or NIBS group, or BSC group, whichever you prefer). Until this day I do not know how you do it, but you always managed to see the best in whatever piece of data I threw in front of you. I don't think I always did a great job of keeping you informed about my whereabouts, but you would manage to tune in to whatever I was rambling about and turn my worry into something good. I don't think I could have asked more from a supervisor.

I would like to say a special word of thanks to my paranymphs, who will not only support me during the day of my defense but have been supporting me already for many years. Helen, I don't even know where to start, I am so grateful that we got to go on this journey together. I could not have wished for a better (and funnier) friend to do this with. Before we started our $\mathrm{PhDs}$ together we hardly knew each other, but now I consider you one of my closest friends. Thanks for all the nonsense, and thanks for all the seriousness.

Sanne, you have always been so down to earth and somehow always willing to listen to me rant over a cup of coffee, which has most certainly helped in keeping me sane over the years. I really admire you as a person and am so happy I got to know you in Japan (of all places!). You get bonus points for introducing some tukker culture into the university by dragging some of us to Zwarte Cross.

During my time in Maastricht I had the privilege of being part of two amazing research groups. Firstly, the brain and emotion group has been great to me over the years. We had our own little suburb on the $3^{\text {rd }}$ floor and it suited us just fine. Thanks to both of my office mates, Aline \& Marta, for being patient with me. Especially Marta, I'm impressed how you managed to be productive even though at some point my side of the office became the designated hang out for all sorts of bored colleagues (I won't name \& shame). Minye, you are such an extraordinary person. I don't know how often I walked into your office opening with the sentence 'this is probably a stupid question, but..', yet you have shown nothing but patience. I'm lucky you'll be joining me in Paris soon! Rebecca, you have been greatly missed since you left! And the group would never be the same without all the other fantastic members, both present and past, so thank you Maarten, Jari, Kiki, Mehrdad, Ruud \& Lisanne.

My second, but equally important, group that welcomed me with open arms was the BSC group. I already creeped into this group during my bachelor research in 2010. Well I guess I 
like you guys, because I haven't left since. Felix, I don't even know what to say. Flying pigs, endless jokes, and yet still an amazing scientific mind (and not afraid to share it). I hope one day we can work together again, you are one of a kind. Lukas, I feel like the two of us could not have been more different, yet I had so much fun hanging out with you (possibly through our mutual love of procrastination). I'm keeping my fingers crossed that you'll win the lottery one day. Teresa, the whole BSC group would not be the same without you. You are so kind and welcoming and relentlessly mean in your jokes at the same time, we all love you to bits. Tom, I have learned so much from you over these years and it has been amazing to have a fellow Nintendo geek around. I also want to thank all of the other amazing members of the BSC group, again past \& present. Thank you so much Jeannette, Charlie, Stefano, Selma, Geraldine, Christianne, Nina, Dennis, and Franzi. Rosanne, I want to give you a special mention; in your acknowledgements you hoped we would get the afterimages project into my thesis, and we totally did it!! Here's to more fun projects that involve sitting in complete darkness for 2 hours.

Of course, I did not spend my entire time isolated in my own little research groups, and I want to thank the entire $\mathrm{CN}$ department for being amazing, warm, welcoming, and a fantastically helpful bunch. A word of special thanks for Linda, you might be the sweetest person I have ever met, and you have impeccable fashion sense. Thanks for sticking with me around uni, in the gym, and elsewhere. Kirsten, I'm going to count you as CN department now. Your determination of trying to get me to adopt some sort of slimy pet is amazing, and on top of that you are a kick-ass scientist. Thanks for all the colleagues that joined the squash group at one time or another, and to all the colleagues who were crazy enough to join my knit nights and actually joined more than once (some of you seemed to genuinely enjoy the knitting, you know who you are).

Ik ben natuurlijk ook heel erg veel dank verschuldigd aan mijn familie. Mam, pap, Shelsy \& Kellan, ik geloof niet dat jullie ooit aan mij getwijfeld hebben en jullie blinde vertrouwen heeft mij altijd geholpen om door te zetten als het allemaal even wat moeilijker werd. Jullie hebben mij altijd het gevoel gegeven dat ik al mijn dromen mocht najagen en ik jullie volledige steun zou krijgen. Die steun heb ik dan ook altijd gevoeld en heeft mij gemaakt tot wie ik nu ben.

Tom, je hebt dit hele proces van helemaal dichtbij mogen meemaken, iets dat geldt voor zowel de ups als de downs. Je hebt in deze tijd echt 'Engelen'-geduld getoond en daar ben ik oneindig dankbaar voor. Dankjewel dat je altijd voor me klaar stond en ik kan niet wachten op ons Parijse avontuur. 
CV \& Publications 

Tahnée Engelen was born in Kerkrade, the Netherlands, on December $1^{\text {st }} 1988$. In 2008 she completed the Gymnasium at College Rolduc in Kerkrade, after which she moved to Maastricht. Here she started her bachelor's degree in biological psychology at Maastricht University, which she successfully completed in 2011. That year she was admitted to the research master programme in cognitive neuroscience at Maastricht University. During her time as a student she worked as a research assistant in the group of Prof. dr. Alexander Sack. In 2012 she moved to Copenhagen, Denmark, to pursue a research internship at the Danish Research Center for Magnetic Resonance (DRCMR) under the supervision of dr. Axel Thielscher. In 2013 she moved back to Maastricht to start her PhD in the cognitive neuroscience department of Maastricht University together with Prof. dr. Beatrice de Gelder and Prof. dr. Alexander Sack. As of May $1^{\text {st }} 2018$, she is working as a postdoctoral researcher in the cognitive neuroscience lab of the École Normale Supérieure in Paris, France, in the group of Prof. dr. Catherine TallonBaudry. 


\section{Publications}

\section{Peer reviewed journal articles}

Engelen, T.*, Schilberg, L.*, ten Oever, S., Schuhmann, T., de Gelder, B., de Graaf, T. A., \& Sack, A. T. (2018). Phase of beta-frequency tACS over primary motor cortex modulates corticospinal excitability. Cortex, 103, 142-152. doi:10.1016/j.cortex.2018.03.001

Poyo Solanas, M., Zhan, M., Vaessen, M., Hortensius, R., Engelen, T., \& de Gelder, B. (2017). Looking at the face and seeing the whole body. Neural basis of combined face and body expressions. Social cognitive and affective neuroscience, 13(1), 135-144.

https://doi.org/10.1093/scan/nsx130

Engelen, T., Watson, R., Pavani, F., \& de Gelder, B. (2017). Affective vocalizations influence body ownership as measured in the rubber hand illusion. PLOS ONE, 12(10): e0186009. doi:10.1371/journal.pone.0186009

Engelen, T., de Graaf, T. A., Sack, A. T., \& de Gelder, B. (2015). A causal role for inferior parietal lobule in emotion body perception. Cortex, 73, 195-202. doi:10.1016/j.cortex.2015.08.013

\section{Manuscripts in revision, under review, and in preparation}

Engelen, T., Zhan, M., Sack, A.T., \& de Gelder, B. (in revision). Dynamic interactions between emotion perception and motor preparation networks for responding to social threat: a combined cTBS-fMRI study.

Zhan, M., Engelen, T., \& de Gelder, B. (in revision). Influence of continuous flash suppression mask frequency on stimulus visibility.

Engelen, T.*, Rademaker, R.L.*, \& Sack, A.T. (in preparation). Gestalt-like reappearance of visual afterimages induced by early visual cortex TMS.

Engelen, T., Zhan, M., Sack, A.T., \& de Gelder, B. (in preparation). Modulation of motor cortex excitability in response to consciously and unconsciously perceived whole body expressions of threat.

Engelen, T.*, Nilsson, S.J.*, Madsen K., Sack, A., Siebner H.R., \& Thielscher, A. (in preparation). Changes in functional connectivity implies a causal role of left dorsal premotor cortex in response selection based on arbitrary visuomotor mapping.

* Authors contributed equally to the work 
